\title{
NUMERICAL ANALYSIS OF FILM COOLING PERFORMANCE OF MICRO HOLES AND COMPOUND ANGLE SISTER HOLES
}

\author{
by
}

\author{
Sana Milud Muftah Abd Alsalam \\ Master of Science, University of Tripoli (2013) \\ Bachelor of Science, University of Tripoli (2005)
}

\author{
A dissertation \\ presented to Ryerson University \\ in partial fulfilment of the \\ requirements for the degree of \\ Doctor of Philosophy \\ in the program of
}

Aerospace Engineering

Toronto, Ontario, Canada, 2019

(C)Sana Milud Muftah Abd Alsalam 2019 


\section{AUTHOR'S DECLARATION}

I hereby declare that I am the sole author of this dissertation. This is a true copy of the dissertation, including any required final revisions, as accepted by my examiners.

I authorize Ryerson University to lend this dissertation to other institutions or individuals for the purpose of scholarly research.

I further authorize Ryerson University to reproduce this dissertation by photocopying or by other means, in total or in part, at the request of other institutions or individuals for the purpose of scholarly research.

I understand that my dissertation may be made electronically available to the public. 


\title{
ABSTRACT \\ NUMERICAL ANALYSIS OF FILM COOLING PERFORMANCE OF MICRO HOLES AND COMPOUND ANGLE SISTER HOLES
}

\author{
Sana Milud Muftah Abd Alsalam \\ Doctor of Philosophy, Aerospace Engineering, Ryerson University, Toronto (2019)
}

In the present research, micro holes and compound angle sister holes have been numerically investigated as two different techniques to enhance the cylindrical hole cooling performance, which suffers from a low cooling performance at high blowing ratio. The numerical analysis is performed over a flat plate model to assess the film effectiveness and the associated flow field at low and high blowing ratios.

The performance assessment of the discrete round micro hole with a $200 \mu \mathrm{m}$ diameter reveals that the micro hole yields the best cooling performance at low blowing ratios, and there is nearly $30 \%$ increase in the overall film cooling effectiveness compared to that of the round macro hole. The flow field results demonstrate the presence of a Counter-Rotating Vortex Pair (CRVP) at a smaller size and less strength, thus, contributed to better spanwise spreading of the coolant jet and lateral film cooling effectiveness. Micro holes present an improvement in the lateral film cooling effectiveness at high freestream turbulence intensity and high blowing ratios.

Computational evaluation of the CFD prediction capability of the sister holes cooling effectiveness using five RANS turbulence models has been carried out as well as an assessment of the effects of the near-wall modeling on the predicted lateral effectiveness. The turbulence models used are realizable k-epsilon, standard k-epsilon, RNG k-epsilon, Reynolds stress model, and Spalart-Allmaras model. It is generally found that realizable k- $\varepsilon$ combined with the enhanced wall treatment provides the best prediction of the numerical results in comparison to the experimental measurements at a low blowing ratio while an underprediction of the lateral performance is found at a high blowing ratio from all examined turbulence models.

The compound angle upstream sister holes (CAUSH) have been proposed as a novel and simple design of the cooling hole whereas the numerical results have shown a notable increase in both centerline and lateral effectiveness for all tested compound angles at all blowing ratios. The anti-counter rotating vortices pair (ACRVP) structure generated from the compound angle 
upstream sister holes has actively controlled the flow field and maintained the coolant jet fully attached to the plate surface while restraining the coolant lift-off at high blowing ratios.

Finally, the influence of the compound angle sister holes streamwise location on the thermal and flow field performance has also been analyzed, whereas three locations: upstream, midstream, and downstream are examined. It is found that the midstream and downstream locations offered a considerable increase in the cooling effectiveness, which is very much dependent on the blowing ratio and the area downstream of the cooling holes. In addition, the optimum centerline effectiveness is obtained by the downstream location, while the best lateral effectiveness is attained through the midstream location. 


\section{ACKNOWLEDGEMENT}

Praise be to Allah the almighty for giving me the success to complete my thesis.

Exactly four years ago, I started my journey of Ph.D. at Ryerson University, and it has been a truly life-changing experience for me. It would not have been possible to complete it without the support and guidance that I received from many people.

Firstly, I would like to express my deepest thanks and sincere gratitude to my thesis advisor, Prof. Bassam Jubran for the continuous support of my Ph.D. study and related research - for his patience, motivation, and immense knowledge. His guidance helped me throughout my research and writing of this thesis. I am very thankful to my supervisor Dr. Jubran, because he introduced me to the field of computational fluid dynamics, and he trained me to be an independent and successful researcher. I could not have imagined having a better mentor and advisor for my Ph.D. study.

Second, I would like to express my special thanks and gratitude to my committee members, Dr. Goetz Bramasfeld, Dr. Hekmat Alighanbari, Dr. Ziad Saghir, and Dr. Martin Agelin-Chaab for their insightful comments and encouragement, which incented me to widen my research from various perspectives.

Third, I want to extend my thanks to the special people I met at Ryerson University, who made me realize the unique value of conducting my study at such a great school. I would like to express my gratitude to the aerospace graduate students colleagues that helped me in the early stages of learning the Fluent Ansys software, Dr. Fadi Mishriky, the Ph.D. student Peter Walker, and last but not least, my colleague and friend Taha Rezzag who is going to start his Ph.D. program this fall and I wish him all the best.

Finally, I would also like to say a heartfelt thank you to my family: my parents, my brothers, and sisters for supporting me and for always believing in me and encouraging me to follow my dreams. Thanks to my pure friend Lois Catalano for her support and encouragement. And finally, to my loved husband and beloved children, who have been by my side throughout this Ph.D., living every single minute of it, and without their faithful support, I would not have had the courage to embark on this journey in the first place. Thank you all. 
I dedicate this thesis to my loving parents

Milud and Miluda, my beloved husband, Khaeri,

my beautiful children:

Ghaiyda,

Abdalsalam,

and Ghasan. 


\section{TABLE OF CONTENTS}

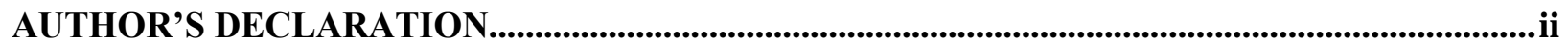

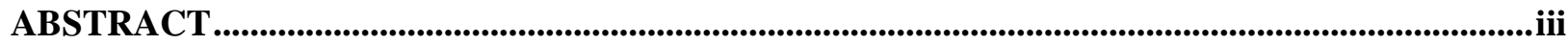

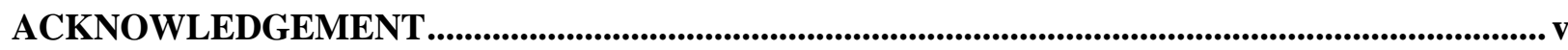

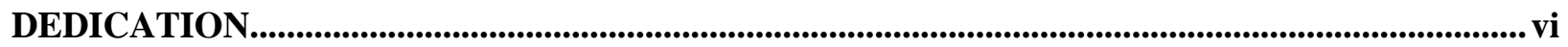

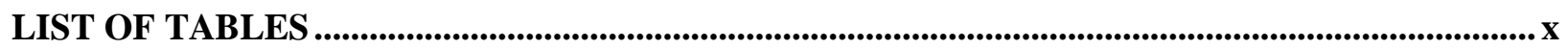

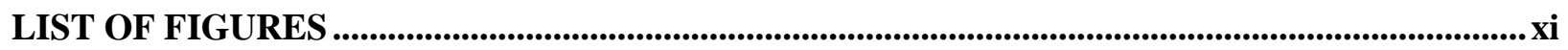

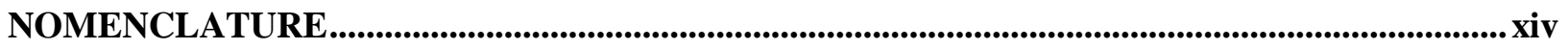

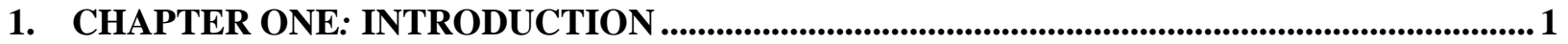

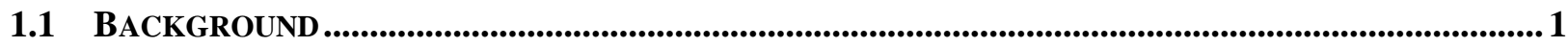

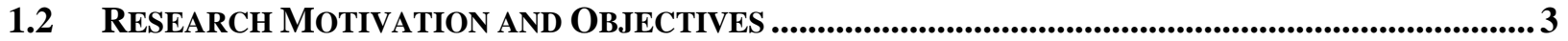

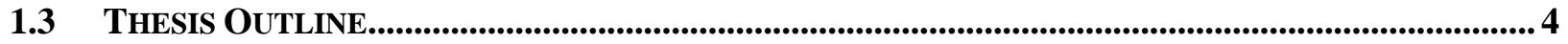

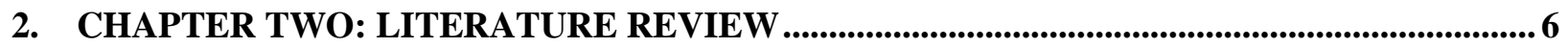

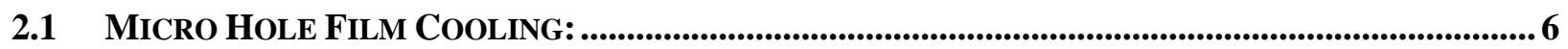

2.2 MACRO HOLE FILM COOLING: .......................................................................................................... 9

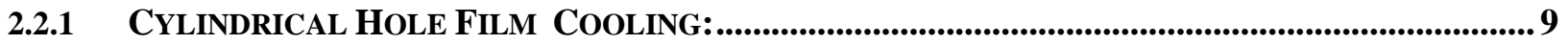

2.2.1.1 film cooling effectiveness at various coolant/freestream and hole configuration parameters .9

2.2.1.2 Cylindrical Hole Flow Physics and Flow Structure:................................................................13

2.2.2 COMPOUND ANGLE Film COOLING OF CYLINDRICAL AND SHAPED HOLE :............................16

2.2.2.1 Compound Angle of Cylindrical Hole:........................................................................................................... 16

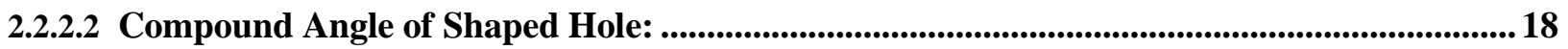

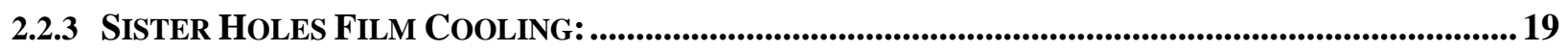

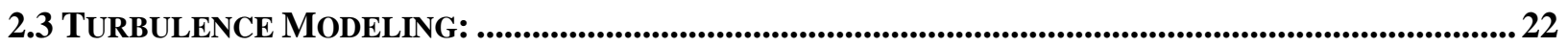




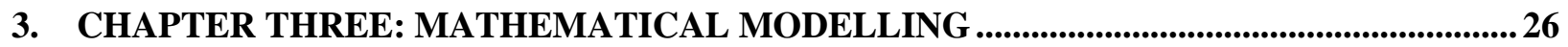

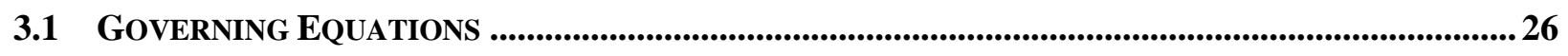

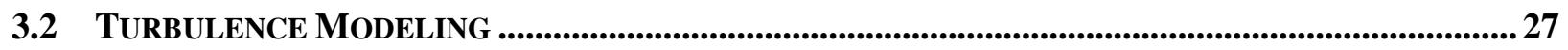

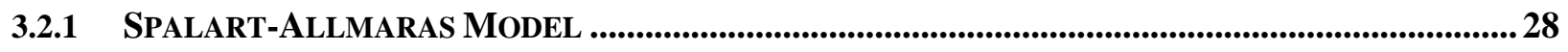

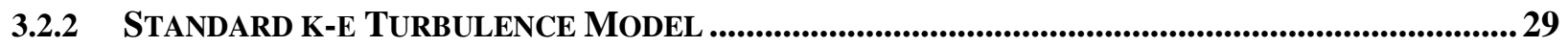

3.2.3 RNG K-E TURBULENCE MODEL .......................................................................................................30

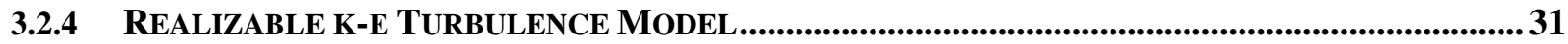

3.2.5 REYNOLDS STRESS MODEL....................................................................................................................32

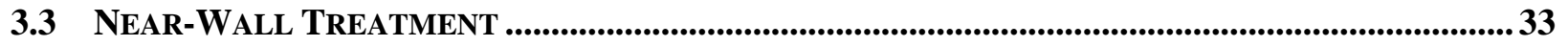

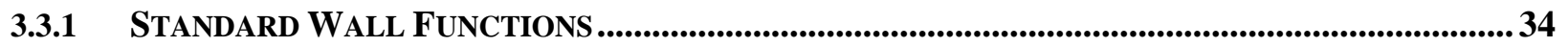

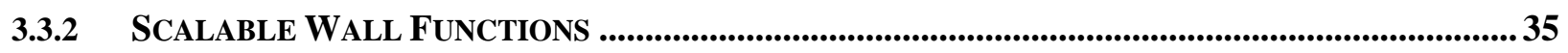

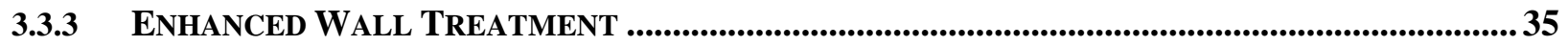

4. CHAPTER FOUR: CYLINDRICAL MICRO HOLE FILM COOLING PERFORMANCE .. 36

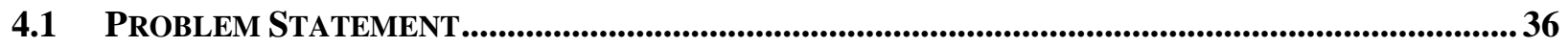

4.2 MESH GENERATION AND COMPUTATIONAL OVERVIEW...................................................................38

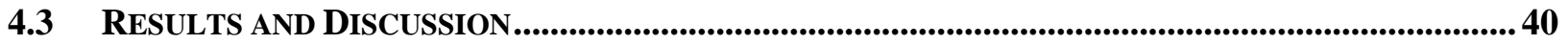

4.3.1 SENSITIVITY ANALYSIS AND NUMERICAL RESULTS VALIDATION.........................................41

4.3.2 THERMAL AND Flow Field RESUlts OF Micro Hole AT VARIOUS BLOWING RATIOS .... 43

4.3.3 THERMAL PERFORMANCE OF MiCRO HOLE AT VARIOUS FrEESTREAM TURbULENCE

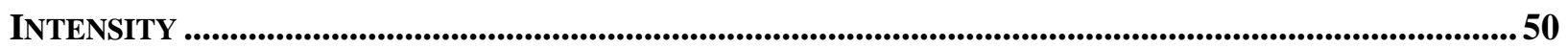

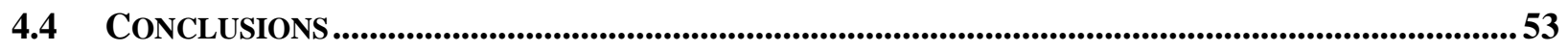

5. CHAPTER FIVE: PREDICTION OF FILM COOLING PERFORMANCE OF UPSTREAM SISTER HOLES USING DIFFERENT TURBULENCE MODELS .................................................55

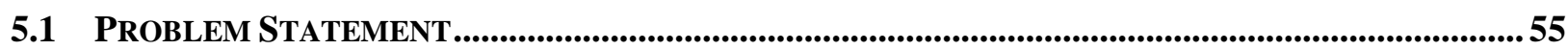

5.2 SIMULATION PARAMETERS AND COMPUTATIONAL OVERVIEW ................................................57

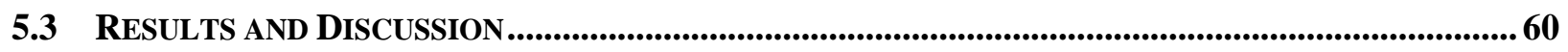

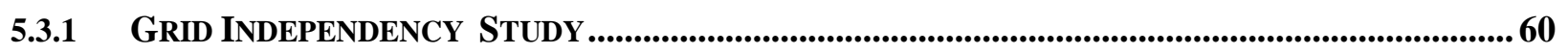


5.3.2 Turbulence Models ANd Near-Wall Treatments Prediction ReSUltS.................... 61

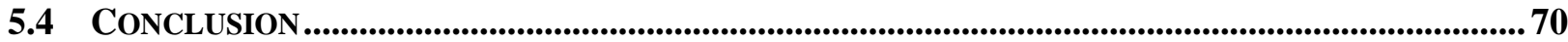

6. CHAPTER SIX: COMPOUND ANGLE UPSTREAM SISTER HOLES FILM COOLING

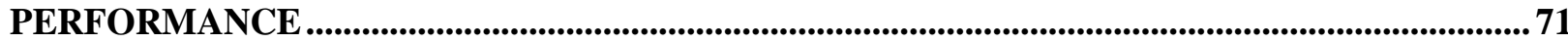

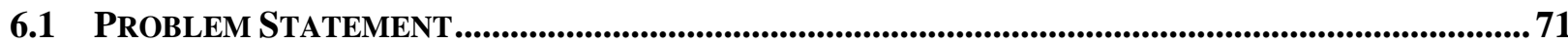

6.2 SIMULATION PARAMETERS AND COMPUTATIONAL OVERVIEW ..................................................... 74

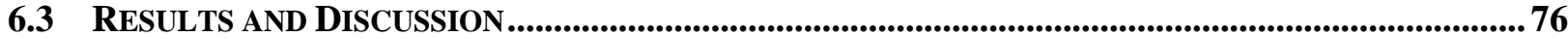

6.3.1 SENSITIVITY STUDY AND NUMERICAL RESULTS VALIDATION ...........................................76

6.3.2 The FILM EFFECTIVENESS RESUltS OF COMPOUND ANGLE UPSTREAM SISTER HOLES

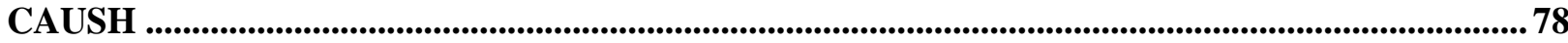

6.3.3 FLOW STRUCTURE OF COMPOUND ANGLE UPSTREAM SISTER HOLES .................................89

6.4 CONCLUSIONS ...............................................................................................................................................97

7. Chapter Seven: Streamwise Location Effect of Compound Angle Sister Holes on Film Cooling

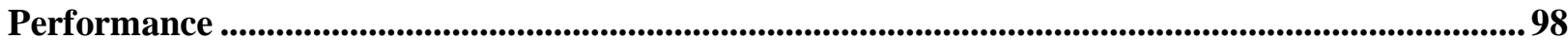

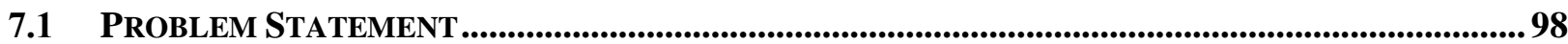

7.2 SIMULATION PARAMETERS AND COMPUTATIONAL OVERVIEW .............................................99

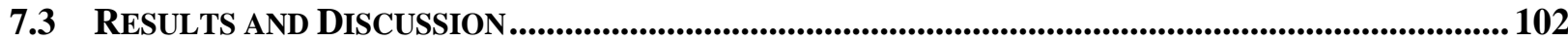

7.3.1 The Film CoOling EFFectiveness Results of The COMPOUND ANGLE Sister Holes

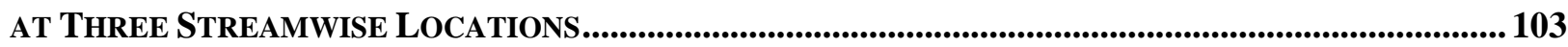

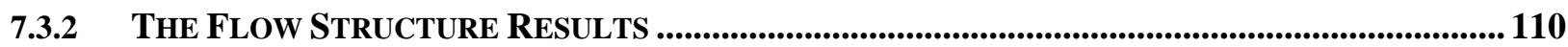

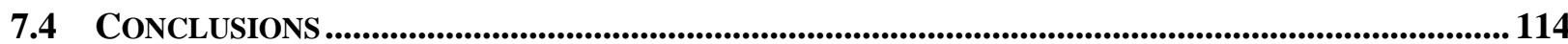

8. CHAPTER EIGHT: CONCLUSIONS AND FUTURE WORK RECOMMENDATIONS .... 116

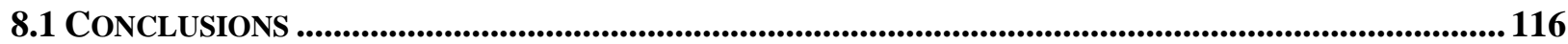

8.2. ConTRIBUTIONS OF THE PRESENT RESEARCH .......................................................................... 118

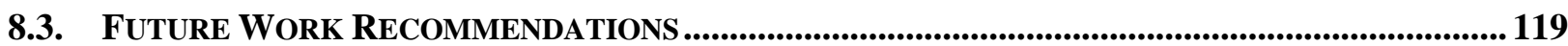

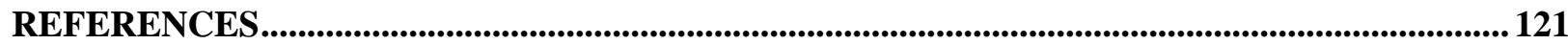




\section{LIST OF TABLES}

Table 4-1-The mainstream and coolant flow parameters. ............................................................38

Table 4-2-The coolant velocity at the plenum inlet...........................................................................38

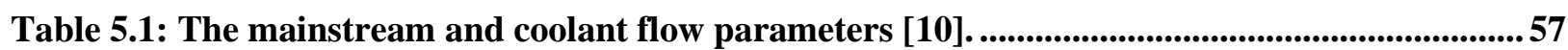

Table 5.2: The coolant velocity at the plenum inlet for the upstream sister holes.............................58

Table 6.1: The dimensions of each CAUSH configuration................................................................ 72

Table 6.2: The mainstream and coolant flow parameters ...............................................................

Table 6.3: The coolant velocity at the plenum inlet. ................................................................................ 75

Table 7.1: The dimensions of the examined CASH configurations......................................................99

Table 7.2: The mainstream and coolant flow parameters .........................................................101

Table 7.3: The coolant velocity at the plenum inlet. ........................................................................ 


\section{LIST OF FIGURES}

Figure 1.1:The chronicle development of turbine inlet temperature [2] .....................................

Figure 1.2: A typical turbine blade cooling techniques: a) Various cooling techniques [4] and (b) film cooling [5]. . .2

Figure 2.1: (a), (b), and (c) the configuration of the three different film cooling hole [7]...........10

Figure 2.2:The configuration of film cooling experimental and the computational domain of

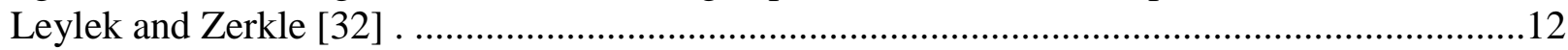

Figure 2.3: The types of vortices structure associated with the jet in crossflow [38]..................14

Figure 2.4:The geometric details of the three nozzle scheme configurations[44] ........................15

Figure 2.5: Top view of the configuration of three cooling holes a) round holes, and b) $15 \mathrm{deg}$. forward expansion holes oriented to the spanwise direction [14] .............................................16

Figure 2.6: The configuration of cooling holes injected with a different arrangement of one and multiple rows of the holes with the simple and compound angle of injection [50]....................17

Figure 2.7 : A top view of different arrangement of sister holes [61][64][62] ............................21

Figure 4.1: a) and b) The 3D model of the micro hole computational domain and boundary

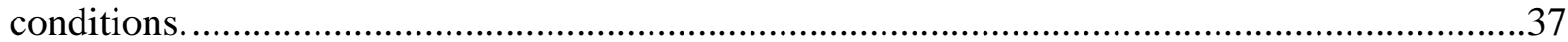

Figure 4.2: The grids generated for the micro round hole.......................................................39

Figure 4.3: The mesh sensitivity for the macro hole shows the centerline film cooling

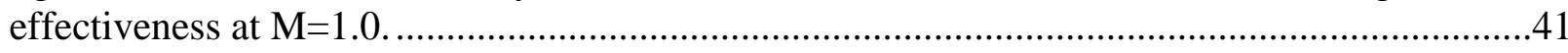

Figure 4.4: The macro hole centerline and lateral film cooling effectiveness prediction at $\mathrm{M}=0.5$ and D.R=2 in contrast to the experimental data of [89]......................................................4

Figure 4.5: The macro hole centerline and laterally averaged film cooling effectiveness at $\mathrm{M}=1.0$ and D.R=2 in contrast to the experimental data of [89]....................................................42

Figure 4.6: The micro hole centerline film cooling effectiveness at $\mathrm{M}=0.25-1.5 \ldots \ldots \ldots \ldots \ldots \ldots \ldots \ldots . . .44$

Figure 4.7: The micro hole laterally averaged cooling effectiveness at $\mathrm{M}=0.25-1.5$.................44

Figure 4.8: The comparison of the averaged laterally averaged film cooling effectiveness between macro and micro hole.

Figure 4.9: Comparison of the average overall film cooling effectiveness between macro and

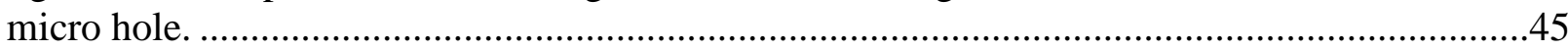

Figure 4.10: The velocity contours at midplane $\mathrm{Z}=0$ at blowing ratio $\mathrm{M}=0.25-1.5 \ldots \ldots \ldots \ldots \ldots \ldots \ldots . .47$

Figure 4.11: The flow structure results at four different locations downstream of the cooling hole at $\mathrm{X} / \mathrm{D}=0,1,3$, and 5 at various blowing rate $\mathrm{M}=0.25-1.5$.

Figure 4.12: The micro hole centerline film cooling effectiveness at various freestream

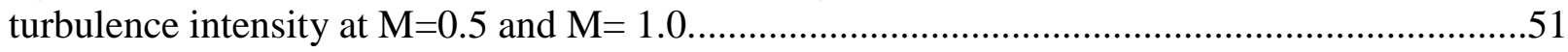

Figure 4.13: The micro hole laterally averaged film cooling effectiveness at various freestream

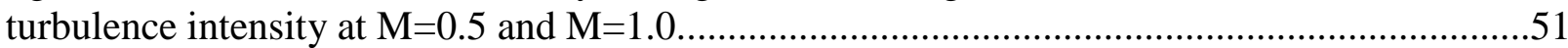


Figure 4.14: The local film cooling effectiveness contours at the plate surface at various..... .52

Figure 5.1: The 3D model of the upstream sister holes a) side view and b) top view. (Not to scale). .56

Figure 5.2: The mesh generated for the upstream sister holes................................................59

Figure 5.3: The 3D computational model boundary conditions ..........................................59

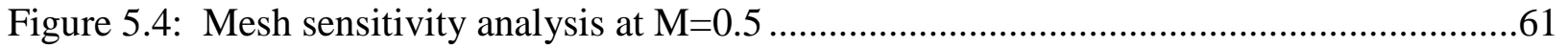

Figure 5.5: The turbulence models prediction of upstream sister holes laterally averaged film cooling effectiveness at $\mathrm{M}=0.5$ and $\mathrm{D} \cdot \mathrm{R}=1.05$.

Figure 5.6: The turbulence models prediction of upstream sister holes laterally averaged film cooling effectiveness at $\mathrm{M}=1.0$ and $\mathrm{D} . \mathrm{R}=1.05$.

Figure 5.7: The near-wall treatments prediction of the lateral effectiveness of the upstream sister holes at $\mathrm{M}=0.5$. .64

Figure 5.8: a) - d) The prediction of the upstream sister holes laterally averaged film cooling effectiveness in contrast to the experimental data of Wu et al. [10] at various blowing ratios. .66 Figure 5.9: The comparisons of the area-averaged film effectiveness to that of Wu et al. [10]..68 Figure 5.10: The comparisons of the film cooling effectiveness distribution at $\mathrm{M}=0.5$ and $\mathrm{M}=1.0$ numerical results (left) and experimental data of $\mathrm{Wu}$ et al. [10](right). ..............................68

Figure 6.1: The computational model for $0^{\circ}$ (CAUSH) (a) top view, (b) side view. (Not to

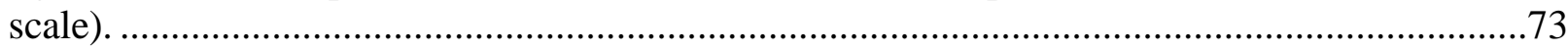

Figure 6.2: A top view focusing on the cooling holes vicinity region for ..............................73

Figure 6.3: The 3D computational domain boundary conditions showing the $45^{\circ} \mathrm{CAUSH}$ case . 75

Figure 6.4: Typical mesh generated for the $0^{\circ}$ (CAUSH) case. (Not to scale) ...........................77

Figure 6.5: The grid sensitivity study and numerical results validation of centerline effectiveness at $\mathrm{M}=0.5$. 79

Figure 6.6: a) and b) The upstream sister holes centerline and lateral film effectiveness at $\mathrm{M}=0.5$ \& D.R=1.2 compared to the CFD results of Ely and Jubran [64][65]. .79

Figure 6.7: a) - (d the centerline film cooling effectiveness of CAUSH at different blowing ratios $\mathrm{M}=0.25-1.5$.

Figure 6.8: temperature contours at three lateral planes; $\mathrm{X} / \mathrm{D}=0,1$, and 3 , and at the plate surface at $\mathrm{M}=0.5$

Figure 6.9: a) - (d The laterally averaged film cooling effectiveness of CAUSH at different blowing ratios $\mathrm{M}=0.25-1.5$.

Figure 6.10: The local film cooling effectiveness for various $\mathrm{CAUSH}$ at $\mathrm{M}=0.25 \& 0.5 \ldots \ldots \ldots . .85$

Figure 6.11: The local film cooling effectiveness for various CAUSH at $\mathrm{M}=1.0 \& 1.5 \ldots \ldots \ldots . .85$

Figure 6.12: Local lateral film effectiveness for various $\mathrm{CAUSH}$ at a) $\mathrm{M}=0.5$ \& b) $\mathrm{M}=1.5 \ldots . .87$ 
Figure 6.13: A Comparison of the overall film cooling effectiveness of (CAUSH) to that of a

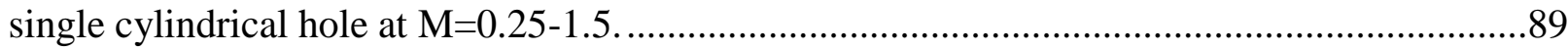

Figure 6.14: Flow structure of the examined CAUSH at $\mathrm{M}=0.25$ and $\mathrm{X} / \mathrm{D}=0,1,3$, and 5........90

Figure 6.15: Flow structure of the examined CAUSH at $M=0.5$ and $X / D=0,1,3$, and 5.........92

Figure 6.16: Flow structure of the examined CAUSH at $\mathrm{M}=1.0$ and $\mathrm{X} / \mathrm{D}=0,1,3$, and 5.........94

Figure 6.17: Flow structure of the examined CAUSH at $\mathrm{M}=1.5$ and $\mathrm{X} / \mathrm{D}=0,1,3$, and 5..........95

Figure 7.1: The side view of the computational model of the upstream $45^{\circ} \mathrm{CASH}$. (Not to scale).

Figure 7.2: A top view magnifies the holes vicinity region for the upstream compound angle sister holes: a) $\beta=45^{\circ} \mathrm{CASH}$, b) $75^{\circ} \mathrm{CASH}$, and c) $90^{\circ} \mathrm{CASH}$, respectively. 100

Figure 7.3: The 3D computational domain boundary conditions applied to the model for the upstream $45^{\circ} \mathrm{CASH}$ case

Figure 7.4: a) -c) The centerline film cooling effectiveness for the $45^{\circ}, 75^{\circ}$, and $90^{\circ} \mathrm{CASH}$, respectively, at $\mathrm{M}=0.5$.

Figure 7.5: a) -c) The centerline film cooling effectiveness for the $45^{\circ}, 75^{\circ}$, and $90^{\circ} \mathrm{CASH}$, respectively, at $\mathrm{M}=1.5$.

Figure 7.6: a) -c) The Laterally Averaged Film Cooling Effectiveness for the $45^{\circ}, 75^{\circ}$, and $90^{\circ}$

$\mathrm{CASH}$, respectively, at $\mathrm{M}=0.5$.

Figure 7.7: a) -c) The Laterally Averaged Film Cooling Effectiveness for the $45^{\circ}, 75^{\circ}$, and $90^{\circ}$ $\mathrm{CASH}$, respectively, at $\mathrm{M}=1.5$.

Figure 7.8: The comparison of a) centerline effectiveness and b) laterally averaged effectiveness for all examined locations and $\mathrm{CASH}$ at $\mathrm{M}=0.5$. 107

Figure 7.9: The comparison of a) centerline effectiveness and b) laterally averaged effectiveness

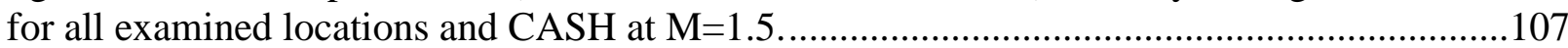

Figure 7.10: The local film cooling effectiveness for various CASH locations at $\mathrm{M}=0.5$........109

Figure 7.11: The local film cooling effectiveness for various CASH locations at $\mathrm{M}=1.5$.......109

Figure 7.12: The flow structure results of $\mathrm{CASH}$ at $\mathrm{X} / \mathrm{D}=0,1$, and $5 \ldots \ldots \ldots \ldots \ldots \ldots \ldots \ldots \ldots \ldots \ldots \ldots . . . . .111$

Figure 7.13: The flow structure results of $\mathrm{CASH}$ at $\mathrm{X} / \mathrm{D}=0,1$, and 5 ...................................113 


\section{NOMENCLATURE}

\section{Alphabetical}

C

cp

D

D.R

E

$\mathrm{H}$

I

$\mathrm{k}$

$\mathrm{k}^{\prime}$

L

L.E

M

$\operatorname{Pr}$

$\mathrm{Re}$

$\mathrm{S}$

$\mathrm{T}$

$\mathrm{t}$

T.E

U

U+

$\mathrm{u}$

$\mathrm{u}_{\tau}$

$\mathrm{V}$

$\mathrm{Y}+$

$\mathrm{X} / \mathrm{D}$

X, Y, Z

\section{Greek}

$\alpha$

$\beta$

$\varepsilon$

$\delta$

$\theta$

$\Theta$

$\kappa$

v

$\mu$
Closure coefficient

Specific heat capacity, J/kg-K

Cooling hole diameter, $\mathrm{m}$

Density Ratio

Total energy

Height

Momentum Flux Ratio

Kinetic energy of turbulence, $\mathrm{m}^{2} / \mathrm{s}^{2}$

Thermal conductivity, W/m-K

Length

Cooling Hole leading edge

Blowing Ratio or Mass Flux Ratio

Prandtl number

Reynolds number

Strain-rate tensor

Temperature, $\mathrm{K}$

Time, sec

Cooling Hole trailing edge

Mean velocity, $\mathrm{m} / \mathrm{s}$

Dimensionless velocity $=\left(\mathrm{U} / \mathrm{u}_{\tau}\right)$

Fluctuating velocity, $\mathrm{m} / \mathrm{s}$

Friction velocity $=\sqrt{ }\left(\tau_{w} / \rho\right), \mathrm{m} / \mathrm{s}$

Velocity, $\mathrm{m} / \mathrm{s}$

Normalized distance from the wall

Downstream distance of the cooling hole

Cartesian coordinate

The angle of streamwise injection.

The angle of compound angle injection.

Turbulent dissipation rate

Kronecker delta

Fluctuating temperature, $\mathrm{K}$

Mean temperature, $\mathrm{K}$

Von Karman constant $(=0.4187)$

Kinematic viscosity $=\mu / \rho, \mathrm{m}^{2} / \mathrm{s}$

Dynamic viscosity, N-s/m2 


$\begin{array}{ll}\rho & \text { Density, } \mathrm{kg} / \mathrm{m}^{3} \\ \left(\mu_{\mathrm{t}} / \mu\right) & \text { Viscosity ratio } \\ \sigma_{\mathrm{k}} & \text { Prandtl number for the kinetic energy } \\ \sigma_{\varepsilon} & \text { Prandtl number for the turbulent dissipation rate } \\ \omega & \text { Specific dissipation rate of turbulent kinetic energy, } \mathrm{k} \\ \eta & \text { Centerline Film cooling effectiveness } \\ \dot{\eta} & \text { Laterally Averaged Film cooling effectiveness }\end{array}$

Subscripts

c

$\infty$

m

Acronyms

ACRVP

CASH

CAUSH

CESH

CFD

CRVP

CVP

DES

DNS

EDM

EESH

EWT

FSTI

GTE

$\mathrm{Kn}$

LD

L/D

LES

MTJ

$\mathrm{P} / \mathrm{D}$

PIV

PSP

RANS

RKE

RNGKE

RSM

Hydraulic

Turbulent

Coolant

Freestream

Micro hole diameter

Anti-counter rotating vortices pairs

Compound angle sister holes

Compound angle upstream sister holes

Circular exit shaped hole

computational fluid dynamics

Counter-Rotating Vortex Pairs

Counter-Rotating Vortex

Detached Eddy Simulation

Direct Numerical Simulation

Electrical discharge mechanism

Elliptical exit shaped hole

Enhanced wall treatment

Freestream turbulence intensity

Gas turbine engine

Knudsen number

Laser Drilling

Cooling Hole Lenght To Diameter Ratio

Large Eddy Simulation

Micro-Tangential Jet

Pitch to Diameter Ratio

Particle Image Velocimetry

Pressure-sensitive paint

Reynolds-Averaged Navier-Stokes

Realizable k- $\varepsilon$ turbulence model

Renormalization group $\mathrm{k}-\varepsilon$ turbulence model

Reynolds stress turbulence model 


$\begin{array}{ll}\text { SA } & \text { Spalart-Allmaras turbulence model } \\ \text { ScWF } & \text { Scalable wall function } \\ \text { SIMPLEC } & \text { Semi-Implicit Method for Pressure-Linked Equations-Consistent } \\ \text { SKE } & \text { Standard k- } \varepsilon \text { turbulence model } \\ \text { SSSH } & \text { Sister-shaped single hole } \\ \text { SWF } & \text { Standard wall function } \\ \text { SUMTJ } & \text { Scaled Up Micro-Tangential Jet } \\ \text { TIT } & \text { Turbine inlet temperature } \\ \text { TLC } & \text { Thermochromic Liquid Crystal Technique }\end{array}$


This Page Intentionally Left Blank

xvii 


\section{CHAPTER ONE: INTRODUCTION}

\subsection{Background}

Gas turbine engines (GTE) are widely used in many applications such as aircraft and marine propulsion systems and electrical power generation. Since the working principle of the (GTE) is based on the Brayton thermodynamic cycle, operating the engine at a high turbine inlet temperature (TIT) enables the engine to achieve substantially high-performance parameters such as the thermal efficiency and the power output.

A typical (TIT) in today's engine reaches around 2000 [K], which is way above the material melting point of the turbine blades [1]. Therefore, in order to prevent the risk of thermal and structural failure, advanced materials technology and sophisticated cooling techniques must be applied. Figure 1.1 shows that for more than six decades, the development of turbine cooling has considerably enabled a significant increase in (TIT) while at the same time a marginal rise has been attained from materials development [2]. For a perspective on these differences, note that an $11\left[{ }^{0} \mathrm{C} /\right.$ year] increase in (GTE) maximum operating temperature was documented as a result of advancements in the thermal management techniques in contrast to $4\left[{ }^{0} \mathrm{C} /\right.$ year $]$ due to the development of alternative materials for gas turbine engines [3]: about one third.

Turbine blades are cooled by means of internal and external cooling techniques. Internal cooling is attained by passing the coolant flow through several enhanced serpentine channels inside the blades and extracting the heat from the outside of the blades. The jet impingement and pin-fin cooling are two examples used in internal cooling [2]. External cooling, also known as film cooling, is one of the most effective cooling methods being applied to turbine blades currently in use. The emphasis of this thesis will be on film cooling, which is done by discharging coolant air from the compressor and injecting it from single/multiple holes on the blade surface to generate a thin layer of cold air, which is several hundred degrees colder than the hot gas. This process then prevents the direct contact of the surface with the coming hot mainstream gases and reduces the heat transfer to it, as shown in Figure 1.2 [4] [5]. 


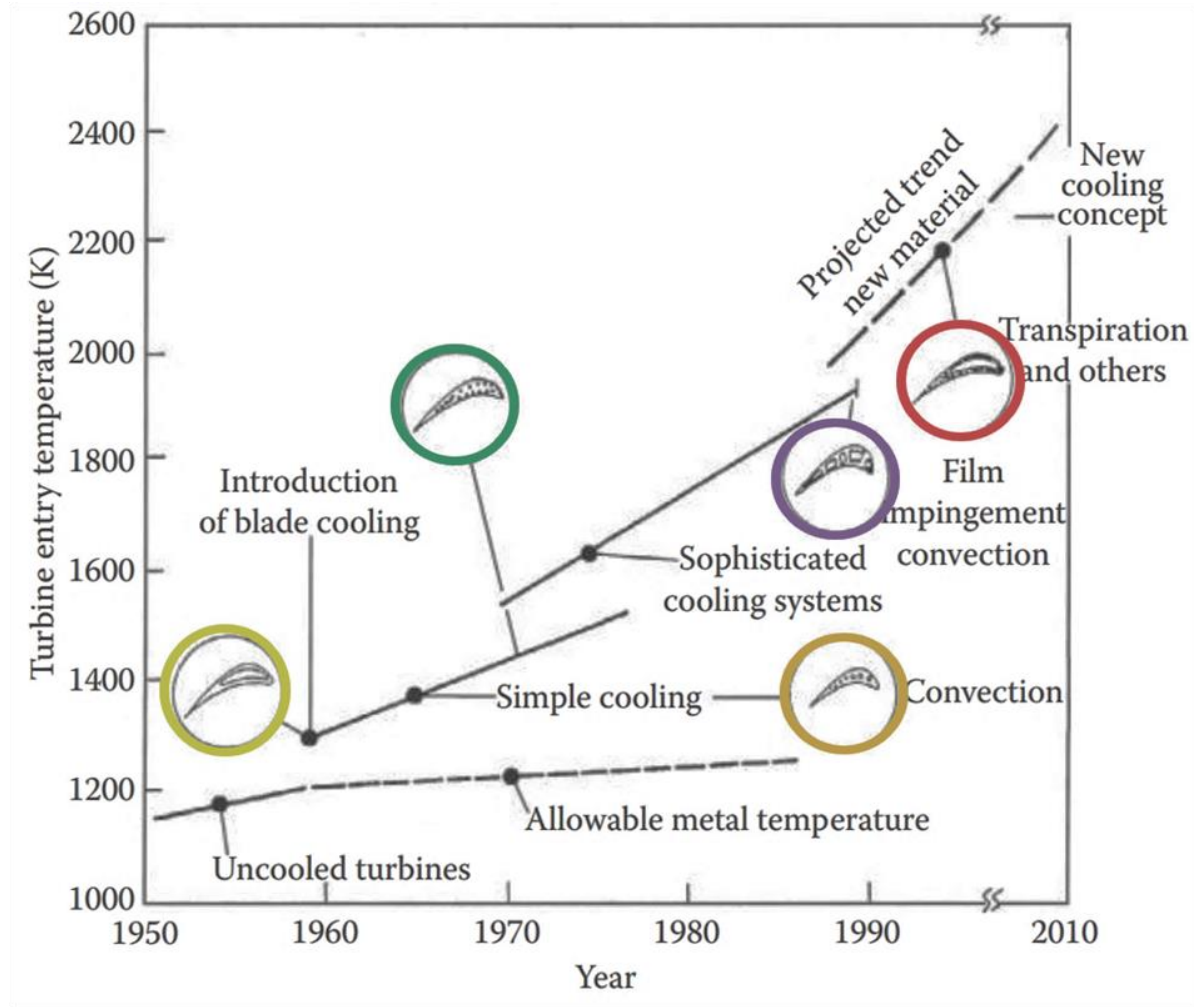

Figure 1.1:The chronicle development of turbine inlet temperature [2].

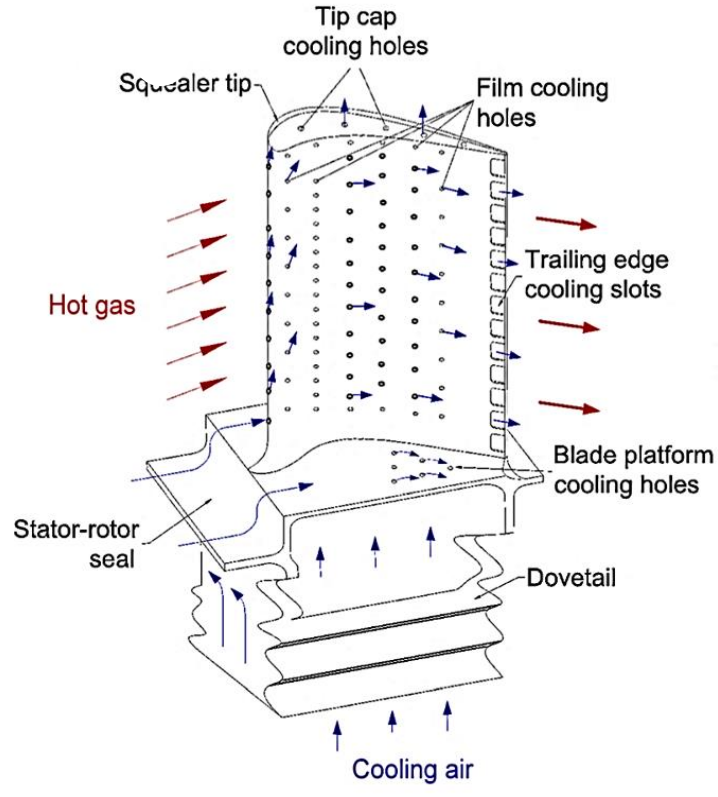

a) Various cooling techniques

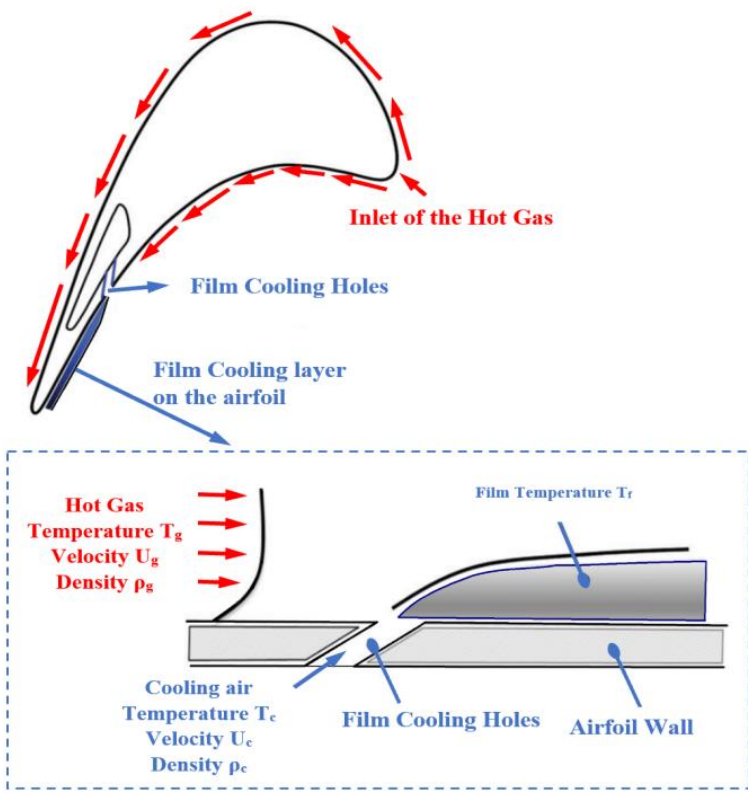

b) Film cooling

Figure 1.2: A typical turbine blade cooling techniques: a) Various cooling techniques [4] and (b) film cooling [5]. 


\subsection{Research Motivation and Objectives}

The increase of gas turbine engines performance is the driven motivation not only to reduce fuel consumption and the subsequent cost benefits but also to increase the specific thrust and mitigate the emissions of $\mathrm{CO} 2$, which is a primary contributor of the increased global warming [5][6]. This increase in performance has been made possible by the continuing development in turbine cooling techniques and manufacturing technologies.

Turbine cooling methods have developed over the years from a simple smooth cooling passage to very complex geometry, including different surfaces and fluid-surface interactions; whereas one of the main aims to enhance turbine cooling is to obtain the highest overall cooling effectiveness with the lowest possible penalty to the thermodynamic cycle performance, which can be achieved by reducing the coolant usage [7]. To give a perspective value of the coolant being used in turbine alone, it reaches approximately $20-30 \%$ of engine compressed air, which presents a severe penalty to the engine performance [8]. Therefore, controlling and optimizing the amount of coolant is crucial while maintaining the temperature of the blade surface within the limit of the maximum allowable material temperature [9]; however, the challenge is how to maintain high cooling performance with less coolant flow at a reasonable manufacturing cost. One approach that was developed is micro hole film cooling with the goal of reducing the coolant flow usage by $50 \%$ through concurrent developments in both design and manufacturing even with a farther increase in (TIT) [7].

Film cooling performance can be significantly improved through the use of the shaped cooling holes, in which many of the recent studies have focused on it to develop and introduce a new cooling hole configurations that offer better performance. On the other hand, some of the proposed shaped cooling holes are very complicated and may not be practical to be applied to real turbine blades. As such, simple and effective cooling holes configuration is always desirable to enhance the thermal protection of turbine blades and the overall engine performance.

Therefore, the research objectives in the present dissertation are;

1. To determine the film cooling performance and to analyze the thermal and flow field of a discrete cylindrical micro film cooling hole with a $200 \mu \mathrm{m}$ diameter on a flat plate model through numerical investigation at various blowing ratios and freestream turbulence intensities. 
2. To evaluate the CFD prediction capability of upstream sister holes film cooling effectiveness through examining five different turbulence models available on ANSYSFluent namely: realizable k-epsilon (RKE), standard k-epsilon (SKE), RNG k-epsilon (RNGKE), Reynolds stress model (RSM), and Spalart-Allmaras model (SA); in addition, to assess the ability of RKE with enhanced near-wall treatment to predict the lateral cooling performance and coolant coverage at low and high blowing ratios. The results will be compared and validated against the only available documented experiment study on sister holes, which was conducted by the research group of $\mathrm{Wu}$ et al. [10].

3. To introduce a novel and simple hole design (compound angle upstream sister holes (CAUSH)) in order to improve the film cooling performance of the cylindrical hole by combining two techniques: Sister holes; (two small round holes placed upstream the primary hole) and a compound angle hole. Sister holes are injected at several compound angles $\beta=0^{\circ}, 45^{\circ}, 75^{\circ}$, and $90^{\circ}$ while the main hole is injected to the streamwise direction at $\alpha=35^{\circ}$ on a flat plate model. The computational analysis of the thermal and flow field along with the flow structure will be conducted using ANSYS-Fluent at various blowing ratios $\mathrm{M}=0.25-1.5$.

4. To evaluate the effect of the streamwise location of the compound angle sister holes (CASH) on the film cooling effectiveness, and to determine the best location of sister holes that offer the optimum cooling effectiveness. Three streamwise locations will be numerically investigated upstream, midstream, and downstream for three compound angle sister holes where $\beta=45^{\circ}, 75^{\circ}$, and $90^{\circ}$ at two blowing ratios $M=0.5$ and 1.5 . The flow structure results will be used to understand the dominant flow field mechanisms responsible for the obtained thermal performance.

\subsection{Thesis Outline}

This thesis is divided into eight chapters. Chapter One, which is the introduction, presents the research motivation and main thesis objectives. In Chapter Two, a comprehensive literature review is conducted on studies that include both numerical and experimental research for the micro holes, compound holes, and sister holes film cooling. Chapter Three demonstrates in detail the thermal and flow field governing equations; Reynolds-averaged Navier-Stokes equations with 
various turbulence models and near-wall treatments. The film cooling effectiveness and the flow field analysis of a single cylindrical micro hole with a $200 \mu \mathrm{m}$ diameter at various blowing ratios and freestream turbulence intensities is covered in Chapter Four. Chapter Five investigates the prediction capability of various turbulence models and near-wall treatments to predict the upstream sister holes cooling performance in contrast to experimental data. The cooling effectiveness, both centerline and lateral, as well as the flow structure of the novel compound angle upstream sister holes (CAUSH), is presented in Chapter Six. The effect of the streamwise location of the compound angle sister holes on film cooling effectiveness and the flow structure is presented in Chapter Seven. Finally, the conclusions, research contributions, and a list of recommendations for future work are provided in Chapter Eight. 


\section{CHAPTER TWO: LITERATURE REVIEW}

Research efforts conducted over the last sixty years; both experimentally and numerically, on film cooling are mainly intended to investigate and analyze the film cooling effectiveness and the related thermal and flow field results at different freestream and coolant flow conditions, as well as cooling hole configuration parameters. The cooling hole configuration parameters include but are not limited to length to diameter ratio, the angle of injection, compound angle injection, the distance between holes, and the shape of the cooling holes. The freestream and coolant flow parameters are the blowing ratio, turbulence intensity, and density ratio. Thereby, in this chapter, the reader will be exposed to the available research on the effect of some of the stated parameters on film cooling performance starting with the micro cooling holes, and then macro cooling holes. Not that, the main focus of this dissertation is on macro cooling holes, particularly the compound angle holes and sister holes and their flow physics. Numerical considerations related to the potential of using various turbulence models to predict film cooling performance is also presented, and finally, the summary of the literature has shown.

\subsection{Micro Hole Film Cooling:}

Unlike the available research on macro hole film cooling, there is very limited research in the open literature on micro hole film cooling, and lately, a growing interest is noticed on evaluating the micro scale film cooling performance as an effective air-cooling technique.

The micro hole cooling was originally proposed to overcome the structural and mechanical strength limitations of the transpiration cooling. In the transpiration cooling, the coolant normally injected to the blade surface through the porous wall with an average of pore diameter of 10-50 $\mu \mathrm{m}$ and a wall thickness of $1 \mathrm{~mm}$ that would achieve a full uniform continuous coolant coverage. Those benefits of the transpiration cooling come at the cost of making the turbine blades structure not sufficient enough to maintain the engine durability goals, and this is the main reason why the transpiration cooling was not matured [11]. Accordingly, with the micro hole cooling, it is expected to achieve the great benefit of film cooling where the coolant remains very close to the blade surface with less mixing with the freestream while maintaining the structure and mechanical strength of the turbine blade. 
Bunker argued that all of the gas turbine blades in todays commercial operation still use the macro hole cooling, but have many weaknesses and limitations and tend to be more complex to achieve high performance[12]. Therefore, moving toward the micro hole cooling would be a simple solution for reducing the amount of coolant while producing a better uniform distribution of coolant, and keeping the coolant close to the surface. Thus, this brings better thermal protection and engine performance. He also stated that the only two problems for the micro hole could be the possibility of blocking or deposition of the cooling hole by the dust and sand particles that come with the cooling fluid and the manufacture challenge for producing this size of hole precisely by an investment casting method. However, with the fast-paced advancements in today's manufacturing and material technology, it is expected to simply produce the micro cooling hole with high accuracy and a reasonable cost using a laser drilling or electrical discharge mechanism $(E D M)$ on a single crystal superalloy whereby the hole diameter can be less than a hundred micrometre [13], [14] as well as by using the additive manufacturing techniques that are considered to be the new revolution in the gas turbine industry [15].

Green performed a preliminary numerical analysis of film cooling effectiveness and the possibility of the hole deposition for a $70 \mu \mathrm{m}$ diameter discrete/row of a cylindrical hole injected at $90^{\circ}$ to the plate surface within a range of the blowing ratio $M=(0.5-2.0)$ and freestream turbulence intensity (5\% and 20\%) [16]. It was reported that the non-existence of the counterrotating vortices structures in the micro hole flow field and increasing the turbulence intensity of the mainstream caused an increase in particle deposition on the plate surface [16].

Sriram and Jagadeesh [17] experimentally investigated the film cooling effectiveness on a nose-cone at hypersonic Mach numbers using a forward-facing array of micro jets (diameter $=300 \mu \mathrm{m}$ ) and compared its cooling performance to a single macro jet (diameter $=2 \mathrm{~mm}$ and 0.9 $\mathrm{mm}$ ). The authors reported that film cooling effectiveness for an array of the micro cooling jets was much better than that of the single macro jet. Also, unlike a single macro jet, the array of micro jets has about $50 \%$ reduction of the surface heat transfer as well as $37 \%$ less coolant consumption.

Gerdroodbary et al. [18] conducted a numerical analysis of film cooling effectiveness for an array of the micro jets at a hypersonic Mach number for the same experimental model as Sriram Jagadeesh [17]. They reported that in addition to the heat load reduction of the multi micro 
jets at the low-pressure ratio, the array of multi-jets had a weak jet momentum and complex 3D structure flow that caused less penetration of multi-jets in the mainstream.

Li et al. [19] experimentally studied film cooling effectiveness from a micro slot at different slot heights of $25 \mu \mathrm{m}, 45 \mu \mathrm{m}$, and $50 \mu \mathrm{m}$ on a flat plate at blowing rates $\mathrm{M}=(1-12.5)$. They found a good film cooling effectiveness from the micro slot, especially with proper control of film slot height and cover plate thickness. The best and the worst performance was attained at a $25 \mu \mathrm{m}$ slot height and cover plate thickness of $200 \mu \mathrm{m}$, and a $45 \mu \mathrm{m}$ slot height with a $100 \mu \mathrm{m}$ cover plate thickness, respectively. The weak performance of the latter scheme was due to the induced strong vortex generated at the back of the cover plate that enhances the mixing of the coolant jet with the mainstream. Moreover, there was a reduction of the coolant air consumption at least twofold than that for the macro scale slot [19].

Film cooling performance evaluation of a micro-tangential jet (MTJ) on the turbine vane that incorporates the benefits of micro jets and tangential injection was experimentally studied by Hassan and Hassan [20] using transient Thermochromic Liquid Crystal technique(TLC). The (MTJ) scheme started with circular cross-section micro round-tube $(600 \mu \mathrm{m})$ and then the hole exit laterally expanded to perform a squared exit at the vane surface. They revealed that MTJ had excellent cooling effectiveness, and this configuration showed a significant lateral spreading of the coolant. Also, the cooling performance from the MTJ on the turbine blade was very close to the case of the 2-D slot over a flat plate, which means that the scheme was able to achieve a performance of the continuous slot while keeping the structural integrity of the blade. On the other hand, the MTJ has unfavourably increased the pressure drop across the scheme exit as well as the thermal stresses at high blowing rates compared to the traditionally shaped holes [20]. Moreover, Hassan [21] pointed out that unlike the macro jet; the micro jet had a unique feature, which was the absence of the surface vortices with a low rate of decay in the centerline velocity and a low level of increase in the turbulence intensity. Thus, it resulted in that the micro jet kept its structure with less mixing and interacting with the mainstream.

Hassan and Hassan [22] performed another experimental investigation of the cooling performance of MTJ using the (PIV) technique at three blowing ratios (0.5, 1 , and 1.5). However, they used a scaled-up model (SUMTJ) for their previous MTJ configuration with a scaling factor of 5 to 1over a flat plate. It was found from the velocity profile results that the coolant jet remained 
attached and parallelled to the plate surface for all blowing ratios even for the far downstream region of the cooling hole exit. Moreover, using lateral expanded angle in this scheme kept the coolant continually uniform in the spanwise direction with less penetration in the mainstream. The vorticity structure results showed the deformation of the CRVP, but its strength was weaker than that of the cylindrical hole [22].

Balasubramaniyan and Jubran [23] initiated a numerical investigation of film cooling performance for a discrete micro round cooling hole that injected at $35^{\circ}$ to the mainstream at two blowing ratios $M=0.5$ and 1.0. They reported that the centerline cooling effectiveness in the closed region to the hole exit was higher than that of the macro jet. They also found that unlike the macro hole, there was a wide spreading of the coolant jet in the spanwise direction in the case of the micro hole due to the weak structure of CRVP. The spreading of the coolant jet became much better with a multiple hole configuration whereas two micro holes injected in the streamwise direction than that of the single micro hole [23]. Balasubramaniyan and Jubran extended their study to include the influence of the cooling hole $\mathrm{L} / \mathrm{D}$ ratio on cooling performance [24]. They found that at an L/D ratio greater than five, the jet became fully developed as well as a slight variation in the performance was reported by further increased in hole L/D ratio [24].

Contrary to macro cooling holes, recently, Ochrymiuk [25] found a substernal decrease in the coolant usage nearly $72.8 \%$ from micro cooling holes that has a $100 \mu \mathrm{m}$ diameter at $\mathrm{M}=1.0$ whilst maintaining the same level of the average value of cooling efficiency.

\subsection{Macro Hole Film Cooling:}

\subsubsection{Cylindrical Hole Film Cooling:}

2.2.1.1 film cooling effectiveness at various coolant/freestream and hole configuration parameters

The film cooling earlier investigations started with a slot, and this slot configuration not only enabled the flow to be two-dimensions but also gave a uniform flow in the spanwise direction. As a result, high film cooling effectiveness was achieved [26], [27]. On the other hand, the high film cooling performance obtained from slot holes came at the cost of manufacturing limitations, structure integrity, mechanical strength, and thermal stress which made the slot 
configuration practically undesirable[26], [28]. Instead, film cooling through a discrete circular cooling hole was used and has been widely applied in cooling a real turbine engine blades.

Goldstein et al. [29] carried out an experimental study to determine the film cooling performance from a cylindrical hole streamwise injected at $35^{\circ}$ and $90^{\circ}$ on a flat plate. They reported that the cooling performance reached its optimum at a blowing ratio of $\mathrm{M}=0.5$ and injection angle of $35^{\circ}$. As the blowing ratio increased further, the cooling effectiveness decreased due to the penetration of the coolant jet into the mainstream and the jet lifting off from the plate surface, which were considered to be the main reasons for reducing the cooling effectiveness of the round hole [29].

Another experimental study was conducted by Goldstein et al. [30] for a discrete/rows of

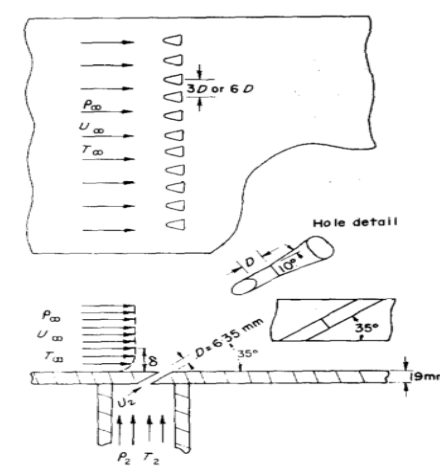

a) Shaped injection
channels b) Long cylindrical injection channels
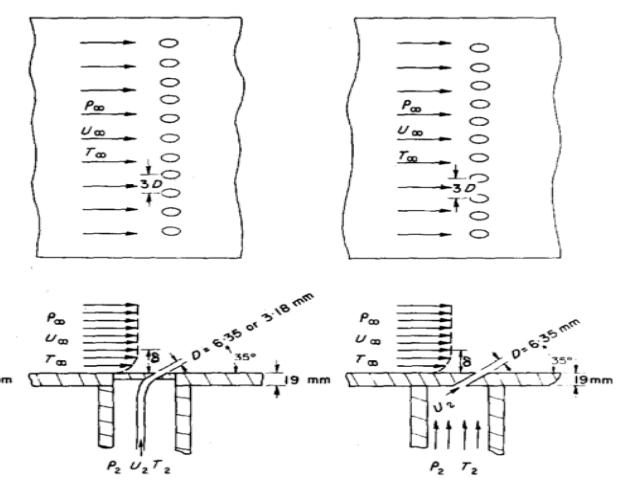

c) Cylindrical injection channels through a thin wall

Figure 2.1: (a), (b), and (c) the configuration of the three different film cooling hole [7].

cylindrical hole(s) to compare its cooling performance with shaped holes; the holes that have diffusion of $10^{\circ}$ near the hole exit on both sides, as shown in Figure 2.1, as well as to evaluate the influence of density ratio on cooling effectiveness. They found that the discrete cylindrical hole has low cooling effectiveness at blowing ratios; $\mathrm{M}>0.5$, and these results agreed well with the previous results of Goldstein [29]. Furthermore, the cooling effectiveness outcomes from a discrete circular and shaped holes were the same at a low blowing ratio $\mathrm{M}=0.5$ while shaped holes gave much better results in which a notable increase in both centerline and lateral effectiveness from a row of the shaped hole at all blowing ratios was documented. Also, it was found that using an approximately dense injected coolant; which is more relevant to the application of the gas turbine, makes the separation of the coolant from the surface occur mainly 
at high blowing ratios comparing it with the case when both coolant and the freestream have the same density.

Cooling hole length to the diameter ratio (L/D) is one of the parameters that have a significant impact on film cooling performance. It is common in the literature to classify the cooling hole with an (L/D) ratio < 3 as a short cooling hole and typically used for film cooling of the combustion chamber and the afterburner liner of the aero-engine while cooling hole with an (L/D) ratio > 3 is classified as a long hole and usually used in the cooling of gas turbine blades [31].

Leylek and Zerkle [32] carried out a numerical study into the effect of cooling hole length to diameter ratio on film cooling effectiveness and flow field. They used the standard $\mathrm{k}-\varepsilon$ turbulence models to predict the film cooling effectiveness and the flow structure. The film cooling experimental setup is shown in Figure 2.2 which consisted of a single row of cylindrical holes inclined at 35 degrees to the freestream direction with (L/D) of 1.75 and 3.5 at a blowing ratio range ( $M=0.5$ to 2$)$. It was found that there is a good agreement between the predicted and experimental results and from a detailed analysis of the complex flow structure inside the cooling hole itself, for both short and long holes, it was revealed that the generation of the counter-rotating vortices that has a predominant effect on cooling effectiveness [32]. In addition, they concluded that a combination of three primary parameters; blowing ratio, hole length to diameter ratio, and the injection angle control the strength of this flow structure [32].

Lutum and Johnson [33] conducted an experimental investigation for the effect of a hole length-to-diameter ratio (L/D) on film cooling effectiveness for a row of cylindrical holes streamwise injected at $35^{\circ}$ at blowing ratios $(0.52 \leq \mathrm{M} \geq 1.52)$ for a range of cooling hole lengthto-diameter ratios of $(\mathrm{L} / \mathrm{D}=1.75,3.5,5,7$, and 18). They found that in the range of $\mathrm{L} / \mathrm{D}$ ratio $(1.75 \leq \mathrm{L} / \mathrm{D} \leq 5)$, film cooling effectiveness decreased by reducing the length to diameter ratio because of the undeveloped flow through the cooling holes and the significant influence of coolant flow injection angle. Moreover, the lowest cooling effectiveness was reported at the hole (L/D) ratio of 1.75, while there were insignificant changes in film cooling effectiveness for

long holes $(\mathrm{L} / \mathrm{D}>5)$.

Aziz and Jubran [34] numerically evaluated the short cooling hole effect on film cooling performance and its thermal and hydrodynamic fields. The model consisted of a single cylindrical 
hole injected at $35^{\circ}$ to streamwise while the hole (L/D ) ratio varied from 1.75 to 8 at two blowing ratios $(0.5-1)$. It was concluded that applying the standard $\mathrm{k}-\varepsilon$ turbulence model with the wall function enhanced film cooling effectiveness prediction precisely at low blowing ratio, and there was a reduction on film cooling effectiveness by decreasing the hole L/D ratio.

Singh et al. [31] experimentally investigated the impact of injection angle ( $\alpha$ ) and (L/D) ratio on film cooling performance of a row of three cylindrical holes over a flat plate at blowing ratios of $M=0.5$ and 1 . The examined angles of injection were $\alpha=\left(15^{\circ}, 30^{\circ}, 45^{\circ}, 60^{\circ}\right.$, and $\left.90^{\circ}\right)$ while the (L/D) ratios were $(1,2,3,4$, and 5). They reported that short holes; (L/D) $\leq 3$, have approximately the same cooling effectiveness whilst long holes; (L/D) $=(4-5)$, showed a major difference in the performance whereby the effectiveness increased with increasing the hole (L/D) ratio [31]. It was also reported that $(\alpha)$ has a major impact on the cooling effectiveness in which the highest performance is attained at $\alpha=15^{\circ}$ for a short hole and $\alpha=45^{\circ}$ for a long hole [31]. Foster and Lampard [35] found that injecting the coolant jet at a small injection angle in the cylindrical hole gave high cooling effectiveness at a low blowing ratio, however, at a high blowing ratio, the large injection angle offered better performance.

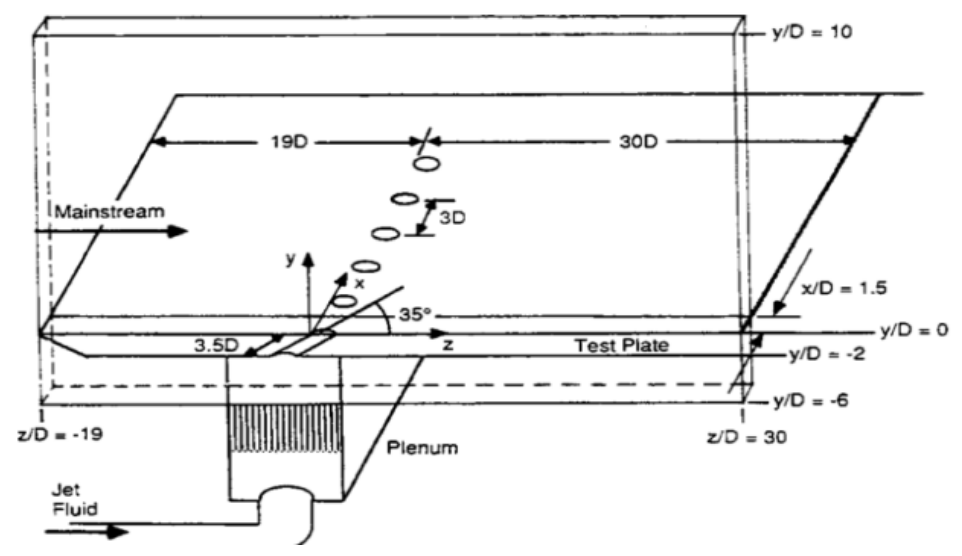

\section{Figure 2.2: The configuration of film cooling experimental and the computational domain of Leylek and Zerkle [32].}

Kohli and Bogard [36] carried out experimental work to investigate the film cooling effectiveness for a row of cylindrical hole inclined at $\alpha=35^{\circ}$ and $55^{\circ}$ at $D \cdot R=2$ and various momentum flux ratio $I=0.08-0.63$. They reported that in contrast to $\alpha=35^{\circ}$, the $\alpha=55^{\circ}$ had shown a minor reduction in the centerline effectiveness at a low momentum flux ratio while at a 
high momentum flux ratio, a degradation on the effectiveness becomes more noticed because of the greater diffusion of the cooling jets near the holes [36].

The influence of injection angle on the local film cooling effectiveness distributions of a discrete cylindrical cooling hole over a flat plate was investigated on the experimental work of Baldauf et al. [37]. The results showed that a steep injection angle at a high blowing ratio leads to uniform lateral coolant distributions as well as high cooling effectiveness compared with a normal coolant injection. On the other hand, the normal injection of the coolant increased the mixing and the penetration $f$ the cooling jet into the mainstream due to the high jet momentum at the hole exit, and thus reducing the cooling effectiveness [37].

Recently, Abd Alsalam and Jubran [38] have found a good centerline and laterally averaged film cooling effectiveness for a cylindrical hole injected at $\alpha=35^{\circ}$ compared with low performance of the large angles of injection: $60^{\circ}$ and $90^{\circ}$ at $\mathrm{M}=0.5$. Furthermore, it was found that the cooling effectiveness of a cylindrical hole decreased as the blowing ratio increased above $M>0.5$ due to the jetting lift-off effect and detachment of the coolant jet from the plate surface which adversely affects the cooling effectiveness.

\subsubsection{Cylindrical Hole Flow Physics and Flow Structure:}

A well-documented feature of the complex 3D-flow structure of a jet in a cross-flow that occurred from the interaction of injected coolant with the freestream as in film cooling of gas turbine blades was reported by Fric and Roshko [39]. The flow structure composed of four types of vortices, as shown in Figure 2.3, specifically jet shear layer vortices, weak vortices, horseshoes vortices, and the pairs of counter-rotation vortices (CRVP). For a simple injection of a round hole, the existence of (CRVP) at the cooling hole exit caused the up wash vortices to push the coolant jet away from the plate surface and force the freestream toward it. This structure of the (CRVP) adversely affects the thermal production of the blade surface and leads to a reduction in cooling effectiveness.

Smith and Mungal experimentally investigated the flow structure and the mixing of the round jet that was normally injected into a uniform cross-flow with varying jet-to-cross flow velocity ratios of 5 to 200 [40]. The results showed that the mixing and penetration of the jet into the freestream increased as the velocity ratio increases and its structure was asymmetric about the plane $\mathrm{z}=0$. 
Also, the development of counter-rotating vortices CVP was reported to occur in the near field region while in the far-field region the CRVP were fully developed [40].

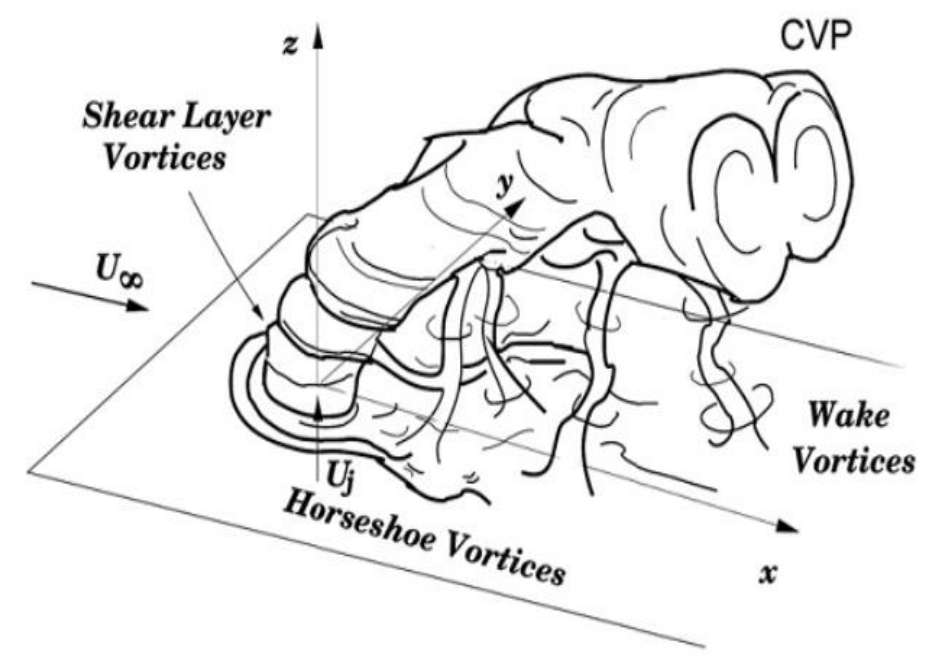

Figure 2.3: The types of vortices structure associated with the jet in crossflow [38].

By comparing the experimental data of a row of short cylindrical cooling holes that were streamwise injected at $\alpha=35^{\circ}$ to the numerical results, Walters and Leylek [41] revealed that the main contributed reason for the existence of counter-rotating vortices flow structure downstream of the hole exit was the dominant streamwise vorticity from the hole boundary layer. The shear existed between the coolant and the freestream, however, had an insignificant impact on cooling flow physics [41]. Unlike the flow structure featured the simple injected round hole that appeared on symmetrical (CRVP), McGovern and Leylek [42] documented that the compound angle injection had a specific flow structure presented on the asymmetric CRVP and this asymmetry increased by increasing the compound angle. At $\beta=90^{\circ}$, the asymmetric CRVP became one single vortex that affected the interaction of the coolant and mainstream and enhanced the lateral spreading of coolant jet [42].

Using the particle image velocimetry (PIV) measurements, Bernsdorf et al. [43] experimentally examined the 3D flow structure of a row of seven cylindrical film cooling holes on the pressure side of the turbine blade at two injection angles $\alpha=30^{\circ}$ and $50^{\circ}$ over a range of flow parameters. The results documented the traditional phenomena of the structure of the 
counter-rotating vortices and the jet lift-off at the high momentum ratios. Furthermore, it was reported that as the blowing ratio increased, the boundary layer thickness became thinner while growth in size and the strength of the rotating vortices perceived, which unfavourably decreased the cooling performance [43].

Li and Hassan [44] numerically presented the effect of the intensity of (CRVP) on film cooling effectiveness of a cylindrical hole and the three new proposed hole configurations called nozzle schemes, shown in Fig 2.4, which were introduced to control the CRVP intensity as well as to isolate the effect of the momentum flux ratio (I). The nozzle configurations A1 and A3 consisted of a couple of semicircular orifice plates placed at the spanwise sides and perpendicular to the streamwise jet direction. However, they had different gap spacing between the orifice plate and the central point of the tube's leading-edge; $0.3 \mathrm{D}$ and $0.0 \mathrm{D}$ for $\mathrm{A} 1$ and A3, respectively. The configuration A2 had a horizontal U-shaped orifice plate with a $0.3 \mathrm{D}$ gap spacing. They revealed that the intensity of the counter-rotating vortices pair was the most critical factor controlling the film cooling effectiveness; specifically, it's x-component, rather than the momentum flux ratio. Also, the results showed that the two nozzles configurations A1 \& A2 effectively reduced the

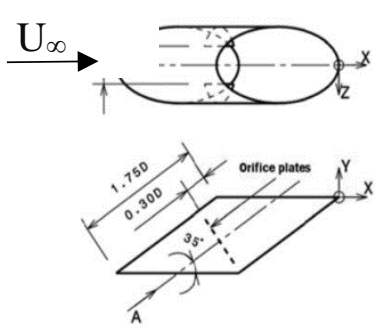

a) Configuration $\mathrm{A} 1$
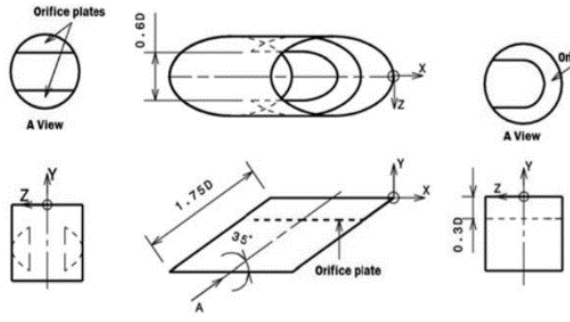

b) Configuration A2
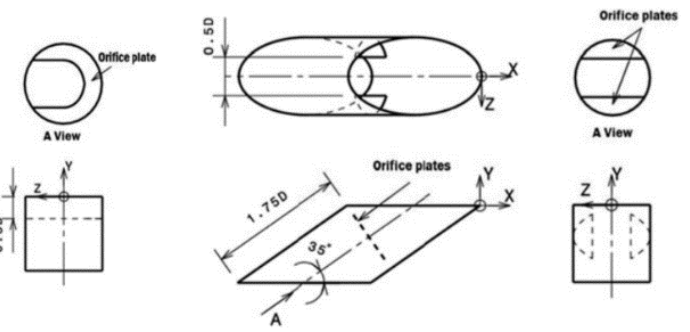

Figure 2.4: The geometric details of the three nozzle scheme configurations[44].

intensity of the CRVP, which resulted in the higher centerline and lateral cooling effectiveness while the best performance was given by the nozzles configurations A1 [44].

Li et al. [45] found from CFD flow structure visualization results that vorticity dynamic developed in the jet-mainstream shear layer near the cooling hole exit was the paramount factor in controlling the deformation and development of CRVP, while the in-tube boundary layer and in-tube vorticity had less impact on CRVP. Furthermore, the results concluded that the CRVP 
strength has a significant effect on the jet lift-off-, attachment, and reattachment of the coolant jet and if the CRVP strength reduced effectively, better cooling performance would be gained [45].

\subsubsection{Compound Angle Film Cooling of Cylindrical and Shaped Hole :}

\subsubsection{Compound Angle of Cylindrical Hole:}

One of the techniques employed to enhance the low film cooling performance of the round holes at a high blowing ratio is compound angle injection. The compound angle of injection ( $\beta$ ) is defined as the angle at which the cooling hole ejects with a primary injection angle with respect to the surface and then orientated via a secondary angle with respect to the mainstream [46]. One of the earliest experimental investigation conducted on the compound angle hole effect on film cooling was performed by Schmidt et al. [47]. They studied a single row of cylindrical holes as a base case and shaped holes; holes have a $15^{\circ}$ forward expanded exit, both injected at $\alpha=35^{\circ}$ and then the two rows of holes oriented with a compound angle $\beta=60^{\circ}$ [47], as shown in Figure 2.5. They found that all examined holes configurations had shown the same spatially averaged cooling effectiveness at a low momentum flux ratio $(I<0.5)$. On the other hand, including the compound angle to the round holes and shaped holes offered a notable increase in the performance specifically at high momentum flux ratio (I > 1) whereas the optimum effectiveness was given by the shaped holes with a $60^{\circ}$ compound angle as a result of improvement in the spanwise distribution of the coolant [47].

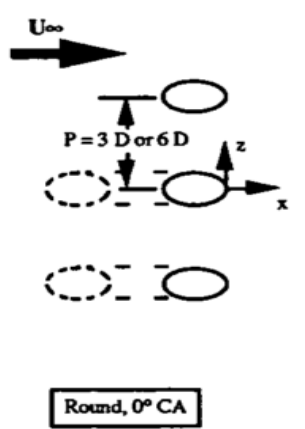

a) Cylindrical holes

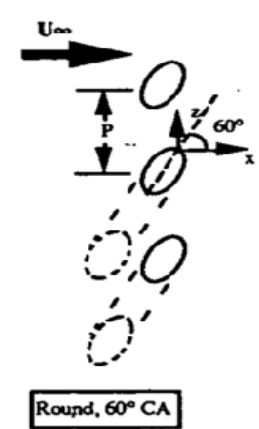

Round, $60^{\circ} \mathrm{CA}$

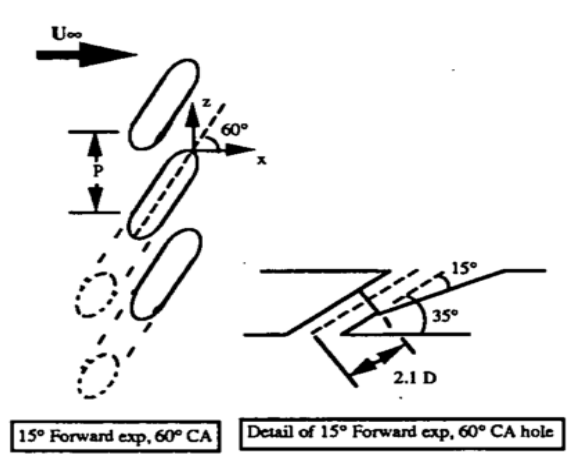

b) $15 \mathrm{deg}$. forward expansion holes.

Figure 2.5: Top view of the configuration of three cooling holes a) round holes, and b) 15 deg. forward expansion holes oriented to the spanwise direction [14].

Ligrani and Lee [48] and Ekkad et al. [49] also experimentally investigated the film cooling effectiveness of a single row of cylindrical holes with compound angle against a row of 
simple injected holes, and it was found that compound angle holes provided better film cooling effectiveness, specifically in the hole near the region compared to streamwise injected holes at the same blowing ratios [48] [49].

Aziz et al. numerically investigated the film cooling effectiveness and its flow field of one and two staggered rows of simple and compound angle holes [50]. The configuration of the computational domain used in this study is shown in Figure 2.6. They concluded that the numerical simulation in most of the cases well predicted the flow field temperature and velocity contours, as well as lateral effectiveness, compared it with the experimental results [50]. Also, in the immediate region downstream of the cooling holes, they predicted that the best film cooling effectiveness was achieved by a blowing ratio of 0.5 for both the simple and compound angles, while an improvement on the spanwise cooling effectiveness was documented for the compound angle injection in contrast to that of simple injected holes [50].

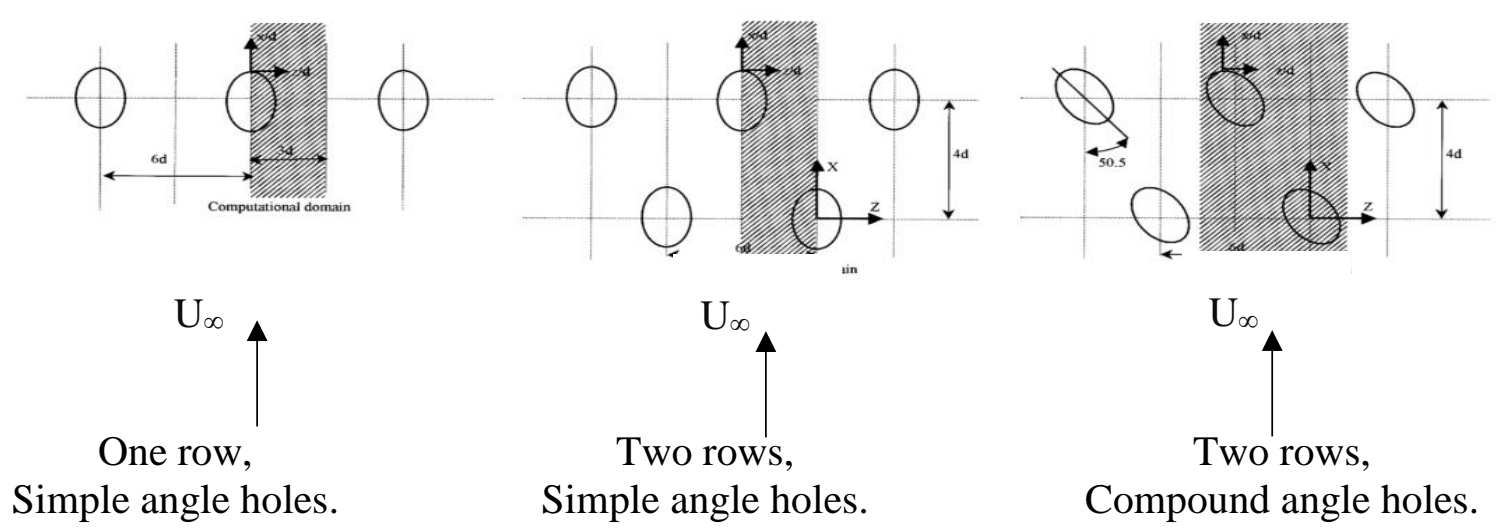

Figure 2.6: The configuration of cooling holes injected with a different arrangement of one and multiple rows of the holes with the simple and compound angle of injection [50].

Sharma and Garg carried out a CFD investigation on the effects of both compound angle and length to diameter ratio on film cooling effectiveness for a single round hole on a flat plate at blowing ratio $(\mathrm{M}=0.5)$ [51]. The range of compound angles was $\beta=\left(0^{\circ}, 30^{\circ}, 45^{\circ}\right.$, and $\left.60^{\circ}\right)$, and (L/D) ratios were $(1,2,3$, and 4). They reported that the lateral coverage of coolant jet in the spanwise direction was increased by increasing the compound angle of injection due to the generation of a single vortex that helped the diffusion of the velocity of the coolant in the lateral that of the simple injected hole for the $\mathrm{L} / \mathrm{D}$ ratios of 1,3 , and 4 , however, at $\mathrm{L} / \mathrm{D}=2$ the effectiveness from a simple injection hole was higher [51]. Also, it was concluded that the combination of a large injection angle with a high (L/D ) ratio increased the mixing of the 
freestream with the coolant jet, and this configuration was inefficient for high cooling performance [51].

Natsui et al. [52] experimentally studied a large spacing compound angle holes for four staggered arrays of cylindrical holes injected at $\beta=45^{\circ}$. The holes spacing were 14.5, and 19.8 times the hole diameter, examined at inclination angles $\alpha=30^{\circ}$ and $45^{\circ}$ at three blowing ratios $\mathrm{M}=0.4,0.8$, and 1.6. They concluded that the blowing ratio and the spacing of the hole have a predominant effect on the film cooling performance, while the angle of the injection effect was second order. Also, the arrays of compound angle jet coolant took a few rows to start interacting and mixing with the coolant of lateral holes at these large hole spacing [52]. In general, the compound angle array of the cylindrical jet was able to provide a significant level of laterally averaged cooling effectiveness at the large hole spacing of $\mathrm{P} / \mathrm{D}=\mathrm{X} / \mathrm{D}=19.8$ [52].

Using a pressure-sensitive paint (PSP) technique, Bashir et al.[53] experimentally evaluated film cooling effectiveness of three rows of compound angle holes in-line and staggered arrangements over a flat plate in which they examined $\beta= \pm 45^{\circ}$ at various blowing ratios $(0.5-$ 1.5). They observed that at a similar $\beta$, the staggered arrangement of compound angle holes

yielded the best cooling performance while the compound angle and holes arrangement observed to have a combined effect on a jet behaviour [53].

More recently, Li et al. [54] experimentally investigated the local distributions of film cooling effectiveness and the laterally averaged effectiveness of a row of simple and compound angle holes over a flat plate surface at different blowing ratios $(\mathrm{M}=0.3-2.0)$ and hole a $(\mathrm{L} / \mathrm{D}=$ 0.5- 5.0), using a high-resolution pressure-sensitive paint (PSP) technique. It was reported that the cooling effectiveness became better with compound angle injected holes for all examined blowing ratios and $(\mathrm{L} / \mathrm{D})$ ratios, while the best performance was attained at $\mathrm{L} / \mathrm{D}=1.0$ for the blowing ratios of $0.5 \leq \mathrm{M} \leq 1.5$ as a result of enhancing the spanwise coolant distribution.

\subsubsection{Compound Angle of Shaped Hole:}

The shape of the cooling hole has been getting considerable attention in the research of film cooling for the last five decades whereas hundreds of new cooling hole configurations proposed and became state of the art due to its superior performance compared to the cylindrical holes. Compound angle injection has also been used with shaped cooling holes to improve further the film cooling effectiveness. Bunker [28] stated that orienting the cooling hole to the axial 
direction was significantly beneficial because it reduced the mixing with the freestream as well as it minimized the mixing losses. A number of publications available on literature documented the advantage of using a compound angle with a shaped hole such as in [47][55] [56] [57]. The numerical study of Brittingham and Leylek [55] found the best lateral cooling effectiveness was obtained by forward- diffused shaped hole with a $60^{\circ}$ compound angle compared to the other examined holes. Baheri et al. [56] computationally found that trenched compound angle shaped hole generated considerably high film cooling protection of both centerline and lateral effectiveness than the other examined configurations. One of the most recent experimental studies was conducted by Haydt and Lynch [57] who found that increasing the compound angle prompted better lateral spreading of the coolant jet and higher lateral effectiveness at high blowing ratios for the triple seven shaped film cooling. It is important, however, to point out to the reader that there are some challenges ultimately facing the practical implementation of the shaped holes with

the compound angle in a real gas turbine engine such as manufacturing difficulties, operability, and cost-effectiveness [58].

\subsubsection{Sister Holes Film Cooling:}

As shown in the previous section of the literature review, film cooling performance can be improved by controlling the flow structure and reducing the strength of the CRVP. Sister holes film cooling is one of the flow control approaches that proved to achieve notable cooling effectiveness from a different arrangement of round holes; two or four small round holes placed at distinct locations from the center of the main manufacturing, which could solve the complexity and manufacture challenges involved in shaped holes. Sister holes specific flow structure appears in the anti-counter rotating vortices pair (ACRVP) produced from the sister hole that worked well to reduce the main hole (CRVP), hence, assessed the adhesion of the coolant jet to the blade surface.

The first studies documented on sister holes was carried out by Javadi et al. [59] and Javadi et al. [60] using combined jets; called " triple jets" with a rectangular cross-section, whereas two small rectangular holes placed downstream of the main hole, and all injected at $\alpha=90^{\circ}$. Their primary goal of this configuration was to control the vortices generated from the main jet and reduce the mixing and the interacting of the combined jets with the mainstream, and it was 
reported that such sister hole arrangements tended to lead to considerable high cooling effectiveness as well as more uniform coolant in the spanwise direction.

Ely and Jubran [61]-[65] conducted series of studies in sister holes film cooling, which were the first to document the sister hole film cooling using multiple round holes, as shown in Figure 2.7. They found a significant improvement in both centerline and lateral cooling effectiveness, not only at low blowing ratios but also at high blowing ratios in comparison to a single round hole [61]-[65].

Further numerical analysis was performed by Ely and Jubran [64] [65] documented that optimal film cooling protection of long sister holes $(\mathrm{L} / \mathrm{D}=5)$ was obtained by placing the sister holes left and right from main hole center, as shown in Figure $2.7 \mathrm{c}$ ), while the maximum performance for short sister holes $(\mathrm{L} / \mathrm{D}=1.16)$ was found via employing four active sister holes, placed 0.75D upstream/downstream the main hole. Moreover, Ely and Jubran [65] compared sister holes film cooling performance of long holes; (L/D) ratio= 5 [64], with that for the short holes; $(\mathrm{L} / \mathrm{D})$ ratio $=1.16$, and they concluded that regardless of the length of the sister hols, a notable increase in film cooling effectiveness was found. However, the main difference identified was in the flow structure generated from short holes that tended to have less defined counterrotating vortex pairs [65][64].

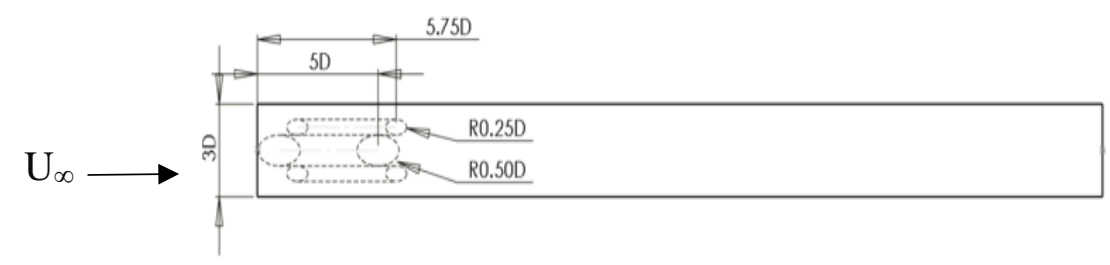

a) Downstream of sister holes

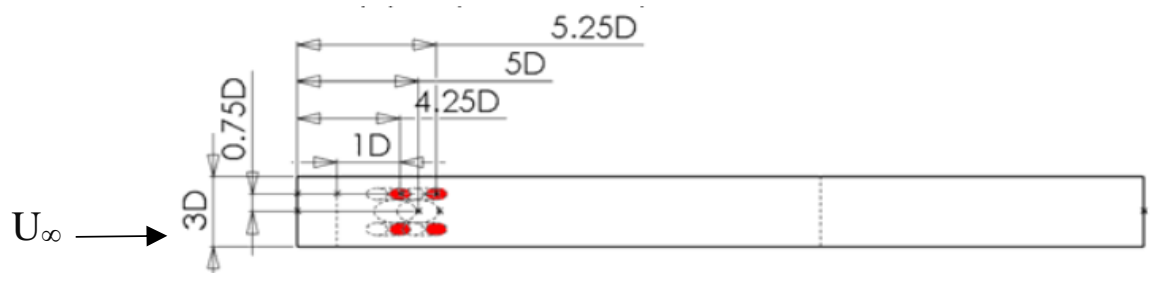

b) Up/downstream sister holes. 


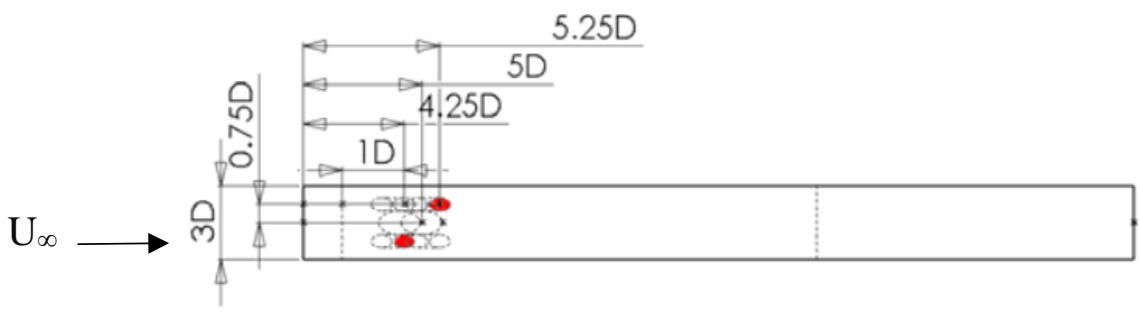

c) Left/ right and up/downstream sister holes

\section{Figure 2.7: A top view of different arrangement of sister holes [61][64][62].}

The impact of sister holes spanwise and streamwise locations on film cooling performance of the up/downstream configuration of sister holes was numerically investigated by Khajehhasani and Jubran [66] in which five sister hole locations were tested in both directions at $\mathrm{M}=0.2-1.5$. It was reported that highest cooling performance was obtained from the modification of the spanwise location of sister holes and the maximum gain in lateral effectiveness was reported at the case of $\mathrm{z} / \mathrm{D}= \pm 1.0$ at $\mathrm{M}=1.0$ [66]. On the other hand, the modification of the streamwise location of sister holes had shown insignificant influence on the cooling effectiveness and the base-case sister holes' location at $\mathrm{x} / \mathrm{D}= \pm 0.75$ yielded the optimal film effectiveness contrary to the other examined cases.

Khajehhasani and Jubran [67] determined that sister holes cooling effectiveness could be increased via an elliptical injection of cooling hole that leads to a circular exit shaped hole in contrast to the base sister holes case [66], in which the steeper injection of a round cooling hole made the shape of the hole to be seen as an elliptical at the plate surface. Better centerline and lateral effectiveness were revealed for the elliptical injection, mostly at high blowing ratios [67].

Khajehhasani and Jubran [68] developed three novel shaped cooling holes based on the sister holes idea through joining discrete sister holes to the primary hole so-called the downstream sister-shaped single hole, the upstream sister-shaped single hole, and the Up/downstream sistershaped single hole. It was reported that the highest film cooling effectiveness was given by the downstream and up/downstream sister-shaped single hole, mainly at high blowing ratios $\mathrm{M}=1.0$ and 1.5, while a slight difference was noticed in the centerline and lateral effectiveness between those two shaped holes [68].

Dai et al. numerically evaluated film cooling effectiveness of various sister holes arrangements while they named parallel-auxiliary holes over a flat plate and found that the case 
of upstream/downstream parallel-auxiliary holes provided the highest film-cooling performance for all examined blowing ratios $(0.5-2.0$. $)[69]$.

\subsection{Turbulence Modeling:}

Numerical simulation of film cooling is done over the years with a progressive improvement in predicting the cooling performance and its associated thermal and flow fields. The challenge, however, still exists because of the complex three dimensions turbulence natural flow field and the involvement of different operating parameters that affect the cooling performance.

Considerable research effort has been made in turbulence modeling prediction of film cooling performance which can be divided into four categories based on turbulence models, namely: 1) Reynolds Averaged Navier Socks RANS- turbulence, 2) large eddy simulation (LES) turbulence, 3) the detached eddy simulation (DES) turbulence, and 4) direct numerical simulation (DNS). The last three models are computationally more expensive and more accurate than the (RANS) and are beyond the scope of this dissertation. Nonetheless, the vast majority of the numerical investigations of film cooling performance are carried out using RANS based turbulence models as they are efficient in capturing the cooling performance as well as they generate the solutions pretty fast.

Leylek and Zerkle conducted one of the first computational studies for a full solution of 3-D Navier-Stokes equations of film cooling that involved a plenum, a discrete short cooling hole, and the mainstream channel [70]. It was documented that they were able to well capture the film cooling effectiveness in contrast to the experimental data, and the flow field results presented the existence of counter-rotating vortices and the local jetting effect from a short cooling hole [70].

Amer and Jubran [71] predicted the cooling performance of two rows of round holes through two different turbulence models, the k- $\omega$ model with its modified version and the standard k- $\varepsilon$ model with its nonisotropic version. It was found that film cooling effectiveness prediction was better using k- $\omega$ turbulence model at high blowing ratios while the standard k- $\varepsilon$ turbulence model and its nonisotropic version well capture the velocity profile at low blowing ratios. 
Walters and Leylek [72] performed a comprehensive 3-D systematic numerical analysis to predict the film cooling performance and have shown the capability of the standard two equations $k-\varepsilon$ turbulence model to predict the performance effectively with less error. Further computational analysis to predict the film cooling effectiveness for a cylindrical hole over the flat plate was performed by Walter and Leylek [41], and it was found that using the two-layer wall treatment instead of wall functions with the standard k- $\varepsilon$ turbulence model well predicted the weak zone in the immediate region of the cooling hole exit even though it increased the computational effort [41].

Azzi and Lakehal [73] reported that the correct spanwise distribution of the temperature field and the vorticial structure strength could only be predicted through the anisotropic eddy viscosity/diffusivity model.

$\mathrm{Na}$ et al. [74] examined the capabilities of three turbulence models realizable k- $\varepsilon$, shearstress transport, and Spalart Allmaras to capture the film cooling effectiveness of a row of inclined circular holes over a flat plate, and it was concluded that a good lateral effectiveness was predicted through the SST model while all the examined models notably overpredicted centerline effectiveness [74].

Hassan and Yavuzkurt [75] investigated four turbulence models standard k- $\varepsilon$, RNG k- $\varepsilon$, realizable k- $\varepsilon$, and standard k- $\omega$, all integrated with EWT to predict both centerline and lateral effectiveness of a discrete and a row of three round holes over a flat plate. It was reported that k$\varepsilon$ turbulence models well predicted the centerline effectiveness where the best numerical result that almost identical to the experimental data was obtained by standard k- $\varepsilon$. On the other hand, the lateral coolant spreading was better predicted by RNG k- $\varepsilon$ and realizable k- $\varepsilon$ models [75].

Harrison and Bogard compared the performance of three variants of RANS turbulence models realizable $\mathrm{k}-\varepsilon$, standard $\mathrm{k}-\omega$, and RSM to predict film cooling effectiveness of a cylindrical hole over a flat plate model [76]. It was concluded that standard k- $\omega$ predicted the lateral film cooling effectiveness very well compared to the experimental data, but this model was not able to capture the centreline cooling performance, which was found to be best predicted by the realizable k- $\varepsilon$ model. Also, the anisotropic Reynolds-stress model RSM did not show any realistic prediction of the coolant spreading among the other models [76]. 
Li et al. [77] employed the algebraic anisotropic turbulence model to improve the prediction of the turbulent eddy viscosity and the scalar diffusivity in the RANS equations. They found that the anisotropic turbulence model more efficiently and accurately predicted the 3D centerline and lateral film cooling effectiveness as well as the flow field at various flow conditions compared to the experimental results.

More recently, Khajehhasani and Jubran [78] examined the ability of four turbulence models; standard $\mathrm{k}-\varepsilon$, realizable $\mathrm{k}-\varepsilon$, RNG $\mathrm{k}-\varepsilon$, and Reynolds-stress model, to predict the centerline effective of a discrete cylindrical hole at a blowing ratio of one. It was reported that the RKE turbulence model outperformed all other examined models and captured very well the centreline effectiveness, the jet lift-off, and attachment in the near hole region. They also examined the near-wall modeling prediction effect using three wall functions, specifically ; enhanced wall treatment, standard wall function, and scalable wall function, while the turbulence model was RKE. It was found that standard wall function provided excellent results of the centerline effectiveness compared to other near-wall approaches in capturing the experimental data.

As shown from this section, multiple RANS turbulence models have been used to predict film cooling performance; however, some of these RANS turbulence models perform better than others, and each RANS turbulence model has its own benefits and drawbacks. It can be concluded that there is no general consensus that exists on the best turbulence model that could be used on film cooling applications as a result of the involvement of wide range of crucial parameters affecting cooling performance( computational model domain size, coolant hole configuration, coolant and freestream operating conditions) to be considered when choosing certain turbulence model [79].

\subsection{The Summary of the Literature Review of Film Cooling:}

Considerable experimental and numerical research efforts are presented in this literature review on film cooling of gas turbine blades to understand the fundamental physics of the thermal and flow fields as well as to highlight new film cooling hole designs and configurations to enhance cooling performance and can be summarized as follows: 
1. Turbine cooling consumes about $20-30 \%$ of the overall compressor bleeding air as a coolant, which is considered a penalty to the engine thermodynamic efficiency. Accordingly, the research on lowering the usage of the coolant jet in film cooling will have great potential in developing new film cooling hole configurations such as a micro hole film cooling.

2. The presented micro hole literature indicated that there is a lack of a detailed analysis of the single micro cylindrical hole thermal and flow fields under engine like operating conditions whereby most of the studied were performed at a low freestream turbulence intensity at limited blowing ratios.

3. The cooling performance of the discrete cylindrical holes is notably diminished by increasing the blowing ratio because of the jet lifting-off. However, cylinder holes remain the most convenient shape of the cooling holes have been used in real turbine blades.

4. The compound angle injection hole enhances both the discrete cylindrical hole and shaped holes film cooling effectiveness as well as the lateral spreading of the coolant jet.

5. Sister holes film cooling are only examined when sister holes injected to the streamwise direction and have shown an improvement in film cooling effectiveness while the existence of ACRVP assists the coolant jet to remain adhesion to the plate surface.

6. There is always a need to develop a simple, effectiveness, and practical cooling holes design that enhances the thermal protection of the turbine blade taking into consideration the manufacturing time and cost. 


\section{CHAPTER THREE: MATHEMATICAL MODELLING}

This chapter presents the governing equations used in computational fluid dynamics (CFD) Reynolds Averaged Navier Stokes equations(RANS), as well as the basic equations of the turbulence models that are available on ANSYS- FLUENT which will be used to evaluate the film cooling effectiveness of the upstream sister holes.

\subsection{Governing Equations}

Reynolds Averaged Navier Stokes (RANS) equations describe the time-averaged form of the governing equations for mean flow quantities. The continuity equation, momentum equation, and energy equation can be written in a cartesian tensor form as:

Continuity equation:

$$
\frac{\partial U_{i}}{\partial X_{i}}=0
$$

Momentum equation:

$$
\rho U_{i} \frac{\partial U_{j}}{\partial X_{i}}=-\frac{\partial p}{\partial X_{j}}+\mu \frac{\partial^{2} U_{j}}{\partial X_{i} \partial X_{i}}-\frac{\partial}{\left(\partial X_{i}\right)}(\rho \overline{u l u j})
$$

Energy equation:

$$
U_{i} \frac{\partial \Theta}{\partial X_{i}}=\alpha \frac{\partial^{2} \Theta}{\partial X_{i} \partial X_{i}}-\frac{\partial}{\partial X_{i}}(\overline{u l \theta})
$$

Whereas $(\rho \overline{u l u j})$ in Eq. (3.2) is the Reynolds stress tensor term, and $(\overline{u l \theta})$ in Eq, (3.3) is the turbulent heat flux term.

In the present study, consistent with the experiments, the simulations are performed for flow over a flat plate. The fluid is considered three-dimensional, incompressible, a Newtonian fluid, turbulent, and steady-state. Furthermore, the working fluid is air and considered to be an ideal fluid. 


\subsection{Turbulence Modeling}

The Reynolds stress tensor term ( $\rho \overline{u l u j})$ and the turbulent heat flux term $(\overline{u \imath \theta})$ shown in equations (3.2) and (3.3), respectively, yield a closure problem for this system of the equations and incorporate the effects of the unresolved turbulent fluctuations (i.e. unresolved by the mean flow equations) on the mean flow. Therefore, turbulence modelling provides the necessary closure by allowing a means for specifying them in terms of mean flow solution quantities.

RANS turbulence models can be divided into two main categories: (1) eddy-viscosity models (based on Boussinesq approximation), which include zero, one, and two-equation models, (2) non-eddy viscosity models or so-called second-moment closure models such as Reynolds stress model that has seven transport equations [80]. The eddy viscosity models invoke the Boussinesq approximation that enforces a linear relationship between the Reynolds stress tensor and the mean strain-rate tensor with a so-called scalar eddy viscosity serving as the isotropic proportionality factor [80].

The Reynolds stresses [80] can be written as:

$$
(\overline{u \imath u \jmath})=\frac{2}{3} k \delta_{i j}-v_{t}\left(\frac{\partial U_{i}}{\partial x_{j}}+\frac{\partial U_{j}}{\partial x_{i}}\right)
$$

Whereas

$\delta_{i j}$ is the Kronecker delta. The turbulent eddy viscosity $\left(v_{t}\right)$ is defined by relating the turbulent kinetic energy $\mathrm{k}$ and the rate of dissipation $\varepsilon$ as follows:

$$
v_{t}=C_{\mu} \frac{k^{2}}{\varepsilon}
$$

The turbulent kinetic energy is defined as

$$
k=\frac{1}{2}\left(\overline{\bar{u}_{\imath}^{2}}+\overline{\grave{u}^{2}}+\overline{{\overline{u_{k}}}^{2}}\right)=\frac{1}{2}(\overline{u \imath u})
$$

The turbulent eddy viscosity displayed in Eq. (3.5) is valid for the standard k- $\varepsilon$, RNG k$\varepsilon$, and realizable k- $\varepsilon$ turbulence models.

On the other hand, the turbulent heat flux $(\overline{u \imath \theta})$ is modeled by applying the simple eddy diffusivity and given as: 


$$
(\overline{u l \theta})=\frac{v_{t}}{P_{r t}} \frac{\partial \Theta}{\partial x_{i}}
$$

Whereas $C_{\mu}$ in Eq. (3.5) $=0.09$ and turbulent Prandtl number in Eq. (3.7) $P_{r t}=0.85$.

The transport equations and a brief overview of turbulence models will be used to investigate film cooling performance in this dissertation are presented in the next subsections, specifically the Spalart-Allmaras model, standard k- $\varepsilon$, RNG k- $\varepsilon$, realizable k- $\varepsilon$, and Reynolds stress model. A detailed description of the governing equations can be found in refs. [67] [80][81] [82].

\subsubsection{Spalart-Allmaras Model}

The Spalart-Allmaras model is a relatively simple one-equation model that solves the transport equation for the turbulent kinematic eddy viscosity. It includes eight closure coefficients and three closure functions [80]. Its defining equations are as follows:

Kinematic Eddy Viscosity

$$
v_{T}=\tilde{v} f_{v 1}
$$

Eddy Viscosity Equation

$$
\frac{\partial \widetilde{v}}{\partial t}+U_{j} \frac{\partial \widetilde{v}}{\partial x_{j}}=c_{b 1}\left[1-f_{t 2}\right] \tilde{S} \tilde{v}-c_{w 1} f_{w}\left(\frac{\widetilde{v}}{d}\right)^{2}+\frac{1}{\sigma} \frac{\partial}{\partial x_{k}}\left[(v+\tilde{v}) \frac{\partial \widetilde{v}}{\partial x_{k}}\right]+\frac{c_{b 2}}{\sigma} \frac{\partial \widetilde{v}}{\partial x_{j}} \frac{\partial \widetilde{v}}{\partial x_{j}}
$$

Whereas the closure coefficients and auxiliary relations in Eq. (3.9) are given by:

$$
\begin{aligned}
& c_{b 1}=0.1355, c_{b 2}=0.622, \quad \sigma=(2 / 3), \text { and } c_{v 1}=7.1 \\
& c_{w 1}=\frac{c_{b 1}}{\kappa^{2}}+\frac{\left(1+c_{b 2}\right)}{\sigma}, c_{w 2}=0.3, \quad c_{w 3}=2 \text {, and } k=0.41 \\
& f_{v 1}=\frac{\chi^{3}}{\chi^{3}+c_{v 1}{ }^{3}}, f_{v 2}=1-\frac{\chi}{1+\chi f_{v 1}} \text {, and } f_{w}=g\left[\frac{1+c_{w 3}{ }^{6}}{g^{6}+c_{w 3}{ }^{6}}\right]^{1 / 6} \\
& \chi=\frac{\tilde{v}}{v}, g=r+c_{w 2}\left(r^{6}-r\right), \text { and } r=\frac{\tilde{v}}{\tilde{S} \kappa^{2} d^{2}}
\end{aligned}
$$




$$
\tilde{S}=S+\frac{\tilde{v}}{\kappa^{2} d^{2}} f_{v 2}, \text { and } S=\sqrt{2 \Omega_{i j} \Omega_{i j}}
$$

The tensor $\Omega_{i j}=\frac{1}{2}\left(\frac{\partial U_{i}}{\partial x_{j}}-\frac{\partial U_{j}}{\partial x_{i}}\right)$ is the rotation tensor and $\mathrm{d}$ is the distance from the closest surface.

Spalart-Allmaras model is known for being suitable for large meshes. It performs well for mildly complex (quasi-2D) external/internal flows and boundary layer flows under a pressure gradient. On the other hand, it performs poorly for 3D flows and flows with strong separation [81][82]. Since film cooling flow field is a 3D flow field featured mainly by CRVP, the SpalartAllmaras might perform poorly. For this reason, this model is not widely used in the literature to predict film cooling performance.

\subsubsection{Standard k- $\varepsilon$ Turbulence Model}

Standard k- $\varepsilon$ turbulence model is the first variant of the k-epsilon model, which is the most common two-equation model used in computational fluid dynamics (CFD) to simulate mean flow characteristics for turbulent flow conditions. This model is a semi-empirical model proposed by Launder and Spalding [83]. The turbulent eddy diffusivity is expressed in terms of two turbulent parameters; the turbulent kinetic energy $(\mathrm{k})$ and the dissipation rate $(\varepsilon)$, which are expressed in equations of (3.15) and (3.16), respectively.

$$
\begin{aligned}
& U_{i} \frac{\partial k}{\partial X_{i}}=\frac{\partial}{\partial X_{j}}\left[\left(v+\frac{v_{t}}{\sigma_{k}}\right) \frac{\partial k}{\partial X_{j}}\right]+\left[-\overline{u_{\imath}^{\prime} u_{\jmath}^{\prime}} \frac{\partial U_{j}}{\partial X_{i}}\right]-\varepsilon \\
& U_{i} \frac{\partial \varepsilon}{\partial X_{i}}=\frac{\partial}{\partial X_{j}}\left[\left(v+\frac{v_{t}}{\sigma_{\varepsilon}}\right) \frac{\partial \varepsilon}{\partial x_{j}}\right]+C_{1 \varepsilon} \frac{\varepsilon}{k}\left[-\overline{u_{\imath}^{\prime} u_{\jmath}^{\prime}} \frac{\partial U_{j}}{\partial x_{i}}\right]-C_{2 \varepsilon} \frac{\varepsilon^{2}}{k}
\end{aligned}
$$

Whereas the standard constants in Eq. (3.15) and (3.16) are given by;

$$
C_{1 \varepsilon}=1.44, C_{2 \varepsilon}=1.92, \sigma_{k}=1.0, C_{\mu}=0.09 \text { and } \sigma_{\varepsilon}=1.3
$$

Standard K- $\varepsilon$ turbulence model is recognizable by its robustness and widely used in film cooling performance numerical evaluation. However, it is also known to perform poorly for 
complex flows involving severe pressure gradients, separation, and strong streamline curvature [81][82].

\subsubsection{RNG k- $\varepsilon$ Turbulence Model}

The Re-Normalisation Group (RNG) k- $\varepsilon$ turbulence model is the second variant of the kepsilon model. The model was derived using a statistical technique called renormalization group theory from the instantaneous Navier-Stokes equations. RNG k- $\varepsilon$ model is similar in form to the standard K- $\varepsilon$ model but includes additional terms and functions in its transport equations for the turbulent kinetic energy and the dissipation rate, as shown in equations of (3.18) and (3.19), respectively [81].

$$
\begin{aligned}
& U_{i} \frac{\partial k}{\partial X_{i}}=\frac{1}{\rho} \frac{\partial}{\partial X_{j}}\left[\left(\alpha_{k} \mu_{e f f}\right) \frac{\partial k}{\partial X_{j}}\right]+\left[-\overline{u_{\imath}^{\prime} u_{\jmath}^{\prime}} \frac{\partial U_{j}}{\partial X_{i}}\right]-\varepsilon \\
& U_{i} \frac{\partial \varepsilon}{\partial X_{i}}=\frac{1}{\rho} \frac{\partial}{\partial X_{j}}\left[\left(\alpha_{\varepsilon} \mu_{e f f}\right) \frac{\partial \varepsilon}{\partial x_{j}}\right]+C_{1 \varepsilon} \frac{\varepsilon}{k}\left[-\overline{u_{\imath}^{\prime} u_{\jmath}^{\prime}} \frac{\partial U_{j}}{\partial X_{i}}\right]-C_{2 \varepsilon} \frac{\varepsilon^{2}}{k}-\frac{R_{\varepsilon}}{\rho}
\end{aligned}
$$

Whereas the additional term shown in the dissipation rate Eq. (3.19) $R_{\varepsilon}$ is identified as

$$
R_{\varepsilon}=\frac{C_{\mu} \rho \eta^{3}\left(1-\frac{\eta}{\eta_{0}}\right)}{1+\grave{\beta} \eta^{3}} \frac{\varepsilon^{2}}{k}, \quad \eta=S\left(\frac{k}{\varepsilon}\right), S=\sqrt{2 S_{i j} S_{i j}}, \quad S_{i j}=\frac{1}{2}\left(\frac{\partial U_{j}}{\partial X_{i}}+\frac{\partial U_{i}}{\partial X_{j}}\right)
$$

While the model constants are given below:

$$
C_{1 \varepsilon}=1.42, C_{2 \varepsilon}=1.68, \eta_{0}=4.38, \quad \grave{\beta}=0.012, \text { and } C_{\mu}=0.0845
$$

A detailed overview of the RNG k-epsilon method can be found in [84].

It is believed that (RNG) $\mathrm{k}-\varepsilon$ turbulence model is suitable in modeling rotating flows and rotating cavities but shown no improvements over the standard model for predicting vortex rotation [82][85]. 


\subsubsection{Realizable k- $\varepsilon$ Turbulence Model}

The Realizable k- $\varepsilon$ turbulence model is the third variant of the k-epsilon model. This model differs from the standard k- $\varepsilon$ model in two ways: (1) it contains an alternative formulation for the turbulent viscosity, and (2) a modified transport equation for the dissipation rate, which has been derived from an exact equation for the transport of the mean-square vorticity fluctuation [81]. The realizable $\mathrm{k}-\varepsilon$ model allows certain mathematical constraints to be satisfied in the Reynolds stresses, which leads to improving its performance [86]. The model transport equations for the turbulent kinetic energy are similar to that of the standard k- $\varepsilon$ model; (Eq. 3.15), while the transport equation of the dissipation rate is defined below in Eq. (3.22) [81].

$$
U_{i} \frac{\partial \varepsilon}{\partial X_{i}}=\frac{\partial}{\partial X_{j}}\left[\left(v+\frac{v_{t}}{\sigma_{\varepsilon}}\right) \frac{\partial \varepsilon}{\partial x_{j}}\right]+C_{1} S_{\varepsilon}-C_{2} \frac{\varepsilon^{2}}{k+\sqrt{v \varepsilon}}
$$

Whereas the additional terms in the dissipation rate Eq. (3.22) are identified as

$$
C_{1}=\max \left[0.43, \frac{\eta}{\eta+5}\right], \eta=S \frac{k}{\varepsilon}, S=\sqrt{2 S_{i j} S_{i j}}, \quad S_{i j}=\frac{1}{2}\left(\frac{\partial U_{j}}{\partial X_{i}}+\frac{\partial U_{i}}{\partial X_{j}}\right)
$$

Unlike the standard and RNG k- $\varepsilon$ models; which has $C_{\mu}$ constant to determine the turbulent eddy viscosity shown in Eq.(3 .5), $C_{\mu}$ for realizable k- $\varepsilon$ models is no longer constant and is found from Eq. (3.24)

$$
C_{\mu}=\frac{1}{A_{0}+A_{s} \frac{k U^{*}}{\varepsilon}}
$$

Where

$$
\begin{gathered}
U^{*} \equiv \sqrt{S_{i j} S_{i j}+\breve{\Omega}_{i j} \breve{\Omega}_{i j}},\left\{\begin{array}{c}
\breve{\Omega}_{i j}=\Omega_{i j}-2 \varepsilon_{i j k} \omega_{k} \\
\Omega_{i j}=\breve{\Omega}_{i j}-\varepsilon_{i j k} \omega_{k}
\end{array}, \mathrm{~A}_{0}=4.04, \text { and } \mathrm{A}_{S}=\sqrt{6} \cos \phi\right. \\
\phi=\frac{1}{3} \cos ^{-1}(\sqrt{6} W), \text { and } W=\frac{S_{i j} S_{j k} S_{k i}}{\sqrt{S_{i j} S_{i j}}}
\end{gathered}
$$

The realizable $k-\varepsilon$ model default constants are given as 


$$
C_{1 \varepsilon}=1.44 \quad C_{2 \varepsilon}=1.9 \quad \sigma_{k}=1.0 \quad \sigma_{\varepsilon}=1.2
$$

Realizable k- $\varepsilon$ model has shown improvement in predictions of the spreading rate of planar and round jets as well as it exhibits superior performance for flows involving rotation, boundary layers under strong adverse pressure gradients, separation, and recirculation [81] [82][85]. It is stated in the literature that realizable $k-\epsilon$ model has a promising capability in predicting film cooling performance and flow field amongst other two equations turbulence models.

\subsubsection{Reynolds Stress Model}

The Reynolds stress turbulence model avoids the isotropic viscosity assumption for Reynolds stresses considered in other models. It closes the RANS equations by solving direct transport equations for the Reynolds stresses $(\overline{u l u j})$ in addition to the equation for the dissipation rate. i.e. for $2 \mathrm{D}$ flow, five additional transport equations need to be solved contrary to seven equations for 3D [81]. This model considers the effects of streamline curvature, swirl, rotation, and rapid changes in strain rate and has greater potential to give accurate predictions for 3D complex flows. However, it is computationally more expensive, and its prediction accuracy is limited by the closure assumptions employed to model various terms in the exact transport equations for the Reynolds stresses [81]. The model transport equations of the Reynolds stresses, dissipation rate, and the constants are shown below in Eqs. (3.28), (3.29), and (3.30), respectively[78].

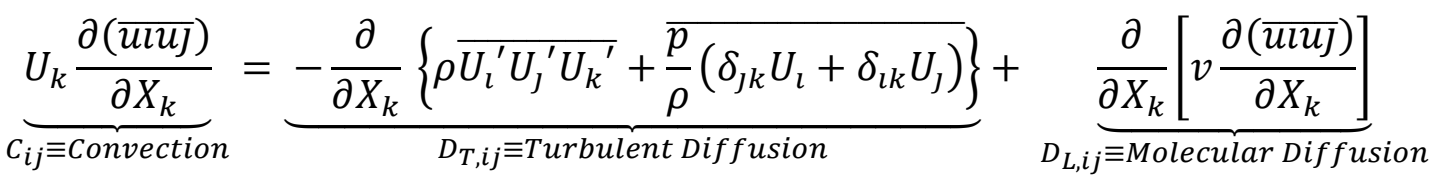

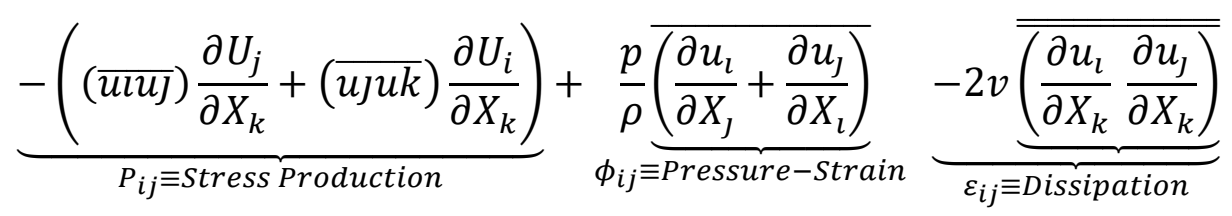

$$
\begin{aligned}
& U_{i} \frac{\partial \varepsilon}{\partial X_{i}}=\frac{\partial}{\partial X_{j}}\left[\left(v+\frac{v_{t}}{\sigma_{\varepsilon}}\right) \frac{\partial \varepsilon}{\partial x_{j}}\right]+C_{1 \varepsilon} \frac{\varepsilon}{k}\left[-\overline{u_{\imath}^{\prime} u_{\jmath}^{\prime}} \frac{\partial U_{j}}{\partial x_{i}}\right]-C_{2 \varepsilon} \frac{\varepsilon^{2}}{k}
\end{aligned}
$$


Where

$$
C_{1 \varepsilon}=1.44 \quad C_{2 \varepsilon}=1.92 \quad \sigma_{\varepsilon}=1.0
$$

\subsection{Near-Wall Treatment}

Turbulent flows are significantly affected by the presence of walls. Near-wall modeling has a major contribution to the accuracy of the numerical solutions whereby the high gradients of the solution of the flow variables such as velocity and temperature occur near the wall region. Hence, a proper presentation of the flow in the near-wall area is very important to the success of simulation and the right prediction of the flow parameters [87].

The flow in near the wall boundary layer is divided into two main regions: (1) the inner layer that consists of the viscous sublayer, buffer layer, and the log-law region, and (2) the outer region. The flow in the viscous sublayer is laminar, and the molecular viscosity has a key role in momentum and heat or mass transfer; however, in the log-law region turbulence dominants [81]. y plus is commonly used to switch between the mentioned regions, and a special attention should be paid to mesh size to get the practical $y+$ within any boundary layer areas in which in the viscous sublayer $(y+<5)$, in the buffer layer $(5<y+<30)$, and in the log-law region $(30<y+<300)$. It is always recommended to void the solution of the flow variables in the buffer layer because no turbulent model is available in this region [81]. On the other hand, it is most recommended for the wall boundary layer to have sufficient overall resolution of the boundary layer to achieve high-quality predictions of the numerical results rather than achieving a particular $y+$ values [8].

Fluent-Ansys offers two common techniques to model the near-wall region. The first one is done by using wall functions; semi-empirical formulas to bridge the viscosity-affected region between the wall and the fully turbulent region and in this case, there is no need to modify the turbulence models to account for the presence of the wall. The second approach is called "nearwall modeling," and is made by resolving the whole region all the way to the wall, including the viscous sublayer, whereas turbulence models are modified to enable the viscosity-affected region to be resolved [81].

Based on the choice of turbulent model, Fluent-ANSYS adapted four different wallfunctions, specifically standard wall functions, scalable wall functions, non-equilibrium wall 
functions, user-defined wall functions, and one choice for near-wall modeling via enhanced wall function[81]. In this dissertation, three wall treatments are used to assess the near-wall treatment effect on predicting film cooling performance of upstream sister holes, which are standard wall function, scalable wall function, and enhanced wall treatments. So, a brief description of those three near-wall treatments will be given below.

For clarification, Fluent-ANSYS uses $\mathrm{y}^{*}$ and $\mathrm{U}^{*}$ instead of $\mathrm{y}+$ and $\mathrm{U}+$ in the set of the equation to specify the near-wall function requirements and features. $\left(y^{*}\right)$ is defined as the dimensionless distance from the wall and is used to check the location of the first node away from a wall, which can be calculated as

$$
y^{*}=\frac{\rho C_{\mu}{ }^{1 / 4} k_{p}{ }^{1 / 2} y_{p}}{\mu}
$$

$\left(\mathrm{U}^{*}\right)$ is defined as the dimensionless velocity and can be calculated as

$$
U^{*}=\frac{U_{p} C_{\mu}{ }^{\frac{1}{4}} k_{p}{ }^{\frac{1}{2}}}{U_{\tau}}
$$

Whereas

$U_{p}$ is the mean velocity of the fluid at the wall-adjacent cell centroid,

$k_{p}$ is the turbulence kinetic energy at the wall-adjacent cell centroid,

$y_{p}$ is the distance from the centroid of the wall-adjacent cell to the wall,

$\mu$ is the dynamic viscosity of the fluid,

$U_{\tau}$ is the friction velocity $=U_{\tau}=\sqrt{\left(\tau_{w} / \rho\right)}$.

\subsubsection{Standard Wall Functions}

The standard wall function is the default option in ANSYS Fluent and is extensively used in industrial flows. It is a semi-empirical formula based on the work of Launder and Spalding [88]. In the viscous sub-layer region $\left(y^{*}<11.225\right)$ and the linear laminar stress-strain relationship is applied and given in the following formula [81].:

$$
U^{*}=y^{*}
$$


In the log-law region $\left(y^{*}>11.225\right)$ is applied and a logarithmic relationship is specified between the dimensionless velocity and dimensionless distance as given below :

$$
U^{*}=\frac{1}{\kappa}\left(E y^{*}\right)
$$

Whereas

$\kappa$ is von Kármán constant $(=0.4187)$

$E$ is an empirical constant $(=9.793)$

\subsubsection{Scalable Wall Functions}

The goal of scalable wall functions is to force the use of the log-law in conjunction with the standard wall function, and a restriction is applied on $y^{* \sim}$ in which it does not allow $y^{*}$ to fall below $\left(y_{\text {limit }}^{*}=11.225\right)$ using the following formula [81].

$$
y^{* \sim}=\max \left(y^{*}, y_{\text {limit }}^{*}\right)
$$

\subsubsection{Enhanced Wall Treatment}

Enhanced wall treatment in the near-wall modeling method combines a two-layer model with enhanced wall functions and needs a very fine mesh near the wall to resolve the whole viscous sublayer all the way to the wall (typically $\mathrm{y}^{+} \approx 1$ ), and then the enhanced wall treatment will be identical to the traditional two-layer zonal model [81].

In terms of the application, standard wall functions are suggested for a wide range of highReynolds number wall-bounded flows because they give appropriate predictions of the numerical solution. However, they become less efficient for the following conditions: low-Reynold-number fluid flow, massive transpiration through the wall (blowing/suction), severe pressure gradients leading to boundary layer separations, and high three-dimensionality in the near-wall region. If that is the case, the near-wall modeling technique must be used combined with the adequate mesh resolution in the near-wall region [81]. 


\section{CHAPTER FOUR: CYLINDRICAL MICRO HOLE FILM COOLING PERFORMANCE}

Numerical investigation of the discrete cylindrical micro hole is presented in this chapter with the emphasis on the influence of the blowing ratio and the freestream turbulence intensity on film cooling performance. The study case features round micro hole with a $200 \mu \mathrm{m}$ diameter over a flat plate. Cooling effectiveness is assessed at four blowing ratios $\mathrm{M}=0.25,0.5,1.0$, and 1.5, while five values of freestream turbulence intensity; FSTI $=0.2 \%, 1 \%, 5 \%, 10 \%$, and $20 \%$, are examined at two blowing ratios, $\mathrm{M}=0.5$ and 1.0 to address its influence on the performance.

\subsection{Problem Statement}

To the best of the author's knowledge, no experimental work has been done yet on the discrete round micro hole film cooling. Therefore, the 3D computational model investigated in this study for the single round micro hole is reduplicated from the experimental study of Sinha et al. [89]. However, the reference hole diameter herein is the diameter of the micro hole $\mathrm{Dm}=200$ $\mu \mathrm{m}$. The model has three main elements; cooling hole, freestream channel, and the plenum. The cooling hole has ( $\mathrm{L} / \mathrm{D}=1.75), \mathrm{P} / \mathrm{D}=3$, and injected to the streamwise at $\alpha=35^{\circ}$. The origin point is located at the cooling hole trailing edge in which the computational model is extended 30D downstream and 19D upstream from the hole leading edge. The freestream channel has a height 10D measured from the plate surface. The plenum has a dimension of $(8 \mathrm{D}, 4 \mathrm{D}$, and $3 \mathrm{D})$ in $(\mathrm{X}, \mathrm{Y}$, and $\mathrm{Z}$ ), respectively. The 3D-geometry is shown below in Figure 4.1. Balasubramaniyan and Jubran [23] have used the same hole configuration used herein; however, the only exception is the location of origin point whereby it was placed at the center of the cooling hole.

Dealing with the micro size of the cooling hole necessitates the validation of the continuum concept and the justification of using the Navier-Stokes equations for the modeling of the micro hole problem on hands. This can be confirmed by calculating the Knudsen number (Kn) [90][91][92]. Knudsen number $(\mathrm{Kn}=\mathrm{K} / \mathrm{H})$ is defined as the ratio between the molecular mean free path of the mainstream and the physical length scale; which is in here the hole diameter $\mathrm{D}=200$ $\mu \mathrm{m}$. It was found that mean free path of air at $(1 \mathrm{~atm})=70 \mathrm{~nm} ; \lambda=70 \mathrm{~nm}$ [93], and when $\mathrm{Kn}<$ 0.001, the continuum concept is valid [90][91]. In this study, the $\mathrm{Kn}$ is found $=(0.00035)$ for the $200 \mu \mathrm{m}$ film hole. Hence the continuum approach is assured, which means that no-slip boundary condition imposed at the solid-gas interface and the fluid is at a local thermodynamic 


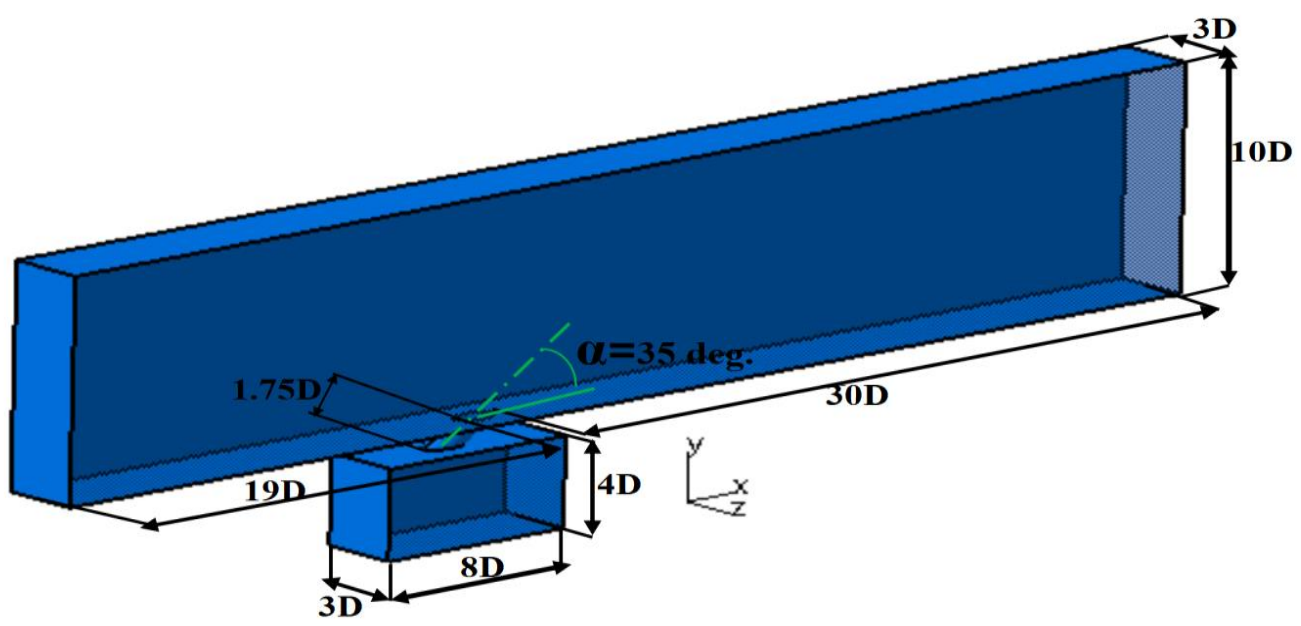

a) The computational domain and its dimensions.

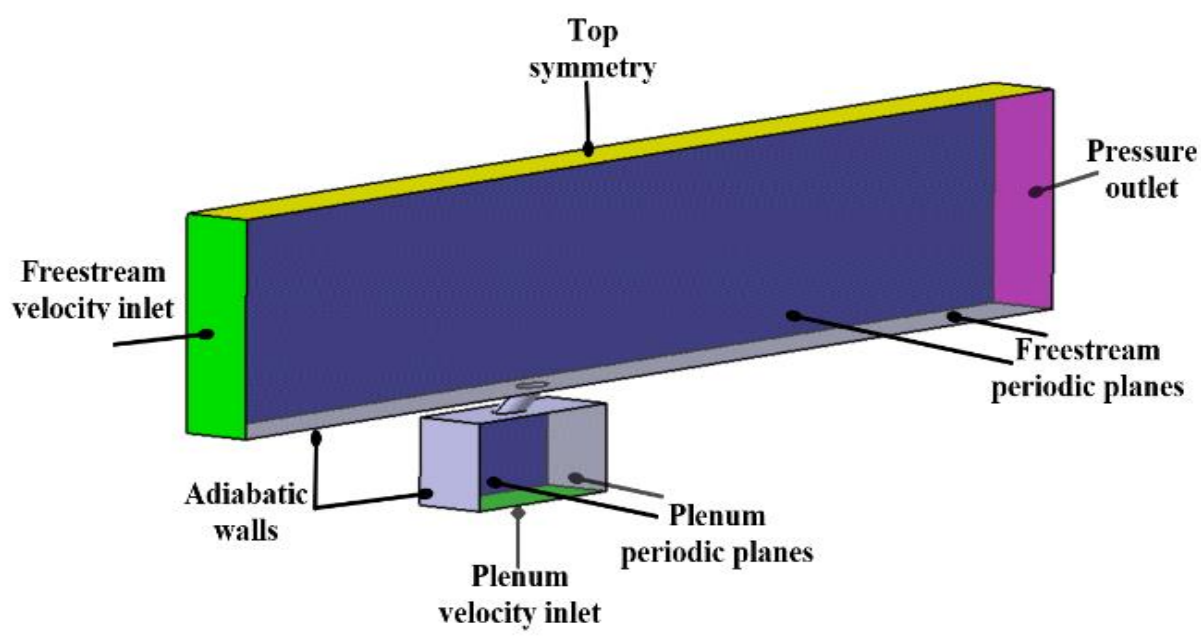

b) The model applied boundary conditions.

\section{Figure 4.1: a) and b) The 3D model of the micro hole computational domain and boundary conditions.}

equilibrium. The coolant and freestream parameters in the numerical simulation are selected to match the experimental data of Sinha et al. [89] listed in Table 4-1. The freestream temperature is $300 \mathrm{~K}$, and the coolant temperature is $150 \mathrm{~K}$ that maintains the flow at a density ratio of two (D.R=2). The turbulent parameters include turbulence intensity of the freestream (FSTI) $=0.2 \%$ and the turbulence intensity for the plenum is $2.0 \%$, and the viscosity ratios $\left(\mu_{t} / \mu\right)=30 \%$ for the plenum and $50 \%$ for the freestream [67]. The model applied boundary conditions are shown in Figure $4.1 \mathrm{~b}$ ). Four blowing ratios are used to evaluate micro hole film cooling performance, $\mathrm{M}$ $=0.25,0.5,1.0$, and 1.5. Noting that the blowing ratio is defined as $\left(\mathrm{M}=\mathrm{D} \cdot \mathrm{R} *\left(\mathrm{~V}_{\mathrm{c}} / \mathrm{V}_{\infty}\right)\right.$, whereas $\left(\mathrm{V}_{\mathrm{c}} / \mathrm{V}_{\infty}\right)$ is the velocity ratio between the coolant and the mainstream. Thus, to get the desired blowing ratio, the freestream velocity is kept constant at $20 \mathrm{~m} / \mathrm{sec}$ while the coolant velocity is 
changed. Table 4-2 illustrates the calculated coolant velocity at the plenum inlet at various blowing ratios. For the cases that employed to analyze the effect of the freestream turbulence intensity on the micro hole cooling effectiveness, other than the previously mentioned value, (FSTI $=1 \%, 5 \%, 10 \%$, and 20\%) are also simulated at two blowing ratios $\mathrm{M}=0.5$ and 1.0.

Table 4-1-The mainstream and coolant flow parameters.

\begin{tabular}{|l|l|}
\hline Property & Value/units \\
\hline Coolant temperature & $150 \mathrm{~K}$ \\
Freestream velocity & $20(\mathrm{~m} / \mathrm{sec})$ \\
Freestream temperature & $300 \mathrm{~K}$ \\
Density ratio (D.R) & 2 \\
Operating pressure & $101.325(\mathrm{kPa})$ \\
\hline
\end{tabular}

Table 4-2-The coolant velocity at the plenum inlet.

\begin{tabular}{|l||c|}
\hline M & Coolant velocity Vc (m/sec) \\
\hline \hline $\mathbf{0 . 2 5}$ & 0.082 \\
$\mathbf{0 . 5}$ & 0.164 \\
$\mathbf{1}$ & 0.327 \\
$\mathbf{1 . 5}$ & 0.491 \\
\hline
\end{tabular}

\subsection{Mesh Generation and Computational Overview}

The grid of the micro cooling hole computational model is performed on ANSYS-ICEM 17.1 using the hexahedral unstructured mesh. The hexahedral cells are generated carefully for the whole domain, and O-grid cells are wrapped around the cooling hole, as depicted in Figure 4.2. Moreover, the mesh is generated to be very fine near the wall and close to the hole region to maintain $(y+<1)$ at all locations to ensure the viscous sublayer is resolved all the way to the wall.

The micro hole cooling performance will be investigated in terms of adiabatic film cooling effectiveness, including both centerlines and laterally averaged film cooling effectiveness, using equations (4.1) and (4.2), respectively. 


$$
\begin{gathered}
\eta_{c}=\frac{\mathrm{T} \infty-\mathrm{Taw}}{\mathrm{T} \infty-\mathrm{Tc}} \\
\bar{\eta}=\frac{1}{\mathrm{l}} \int_{0}^{\mathrm{l}} \eta \mathrm{dz}
\end{gathered}
$$

Whereas $\mathrm{T}_{\infty}, \mathrm{T}_{\mathrm{aw}}$, and $\mathrm{T}_{\mathrm{c}}$ are the temperature of the freestream, the adiabatic wall, and the coolant, respectively. (l) is the length of the lateral distance in the spanwise direction.

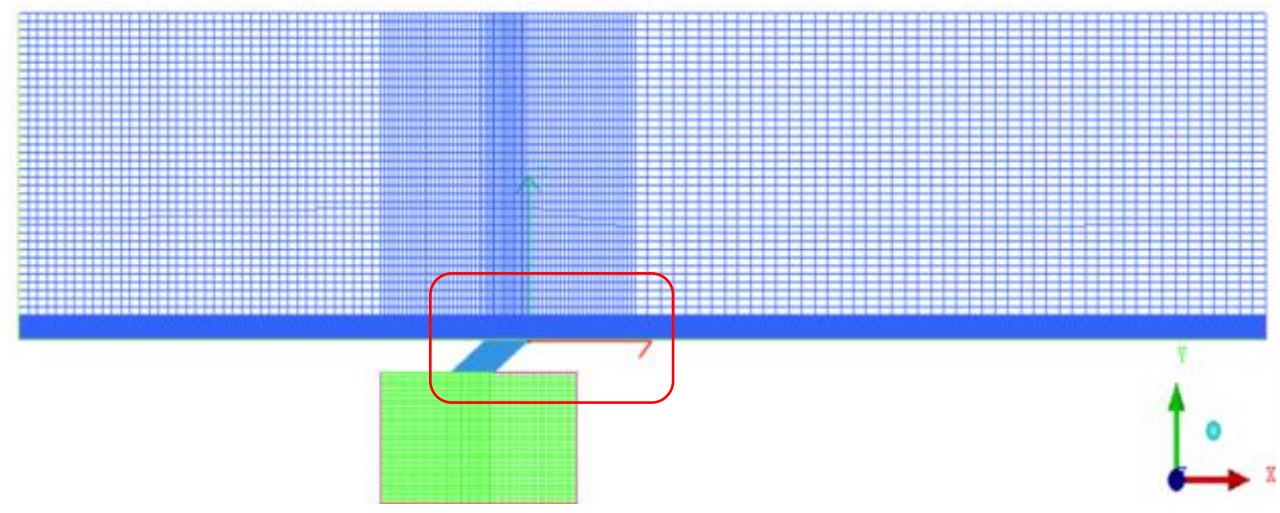

a) A front view of the mesh.

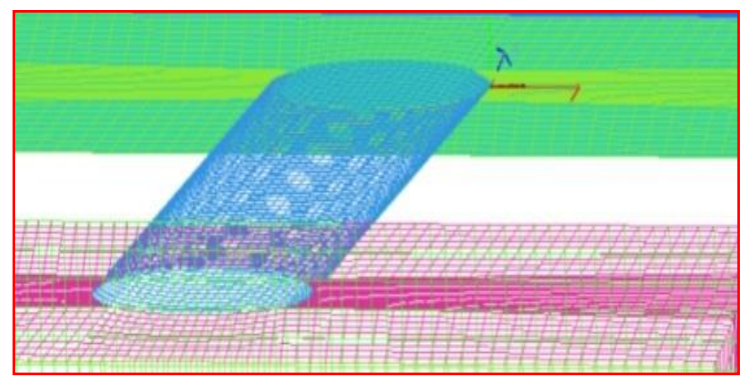

b) The mesh generated for the cooling hole vicinity region.

Figure 4.2: The grids generated for the micro round hole.

The simulation cases of the micro cooling hole are run in ANSYS Fluent 17.1 commercial package. The analysis is carried out as a steady-state, and RANS equations are solved based on the second-order upwind scheme. Because of the turbulence nature of film cooling, appropriated selection of the turbulence model is essential to capture the film-cooling thermal and flow fields. It has been found that the cylindrical cooling hole can be well captured using the realizable $\mathrm{k}-\varepsilon$ turbulence model as documented on the study of Zhang and Hassan [94], Balasubramaniyan and Jubran [23], Khajehhasani and Jubran [78], and Wu et al.[10]. Therefore, the turbulent of the flow is presented thought the realizable $\mathrm{k}-\varepsilon$ (RKE) turbulence model, while the enhanced wall treatment (EWT) is selected for near-wall modeling. The SIMPLEC algorithm is applied as a 
discretized scheme to solve the velocity-pressure coupling. Consistently with the experiment, the working fluid is air, which is considered as an incompressible and ideal gas flow. The numerical results are considered to reach convergence when the normalized residuals for the continuity equation and all velocity components reaching $\left(1 \times 10^{-4}\right)$, while also the normalized residuals of energy equation reaching $\left(1 \times 10^{-6}\right)$.

It should be noted that the cylindrical macro cooling hole is also investigated herein to validate the numerical results as well as for comparison purpose. The $3 \mathrm{D}$ model, in this case, is a discrete round hole of the same experimental setup of Sinha et al. [89] with a hole diameter $(\mathrm{D}=12.7 \mathrm{~mm})$. In addition, the procedures of mesh generation and numerical setup are identical to that stated above for the micro hole case.

It is significant to point out why the experimental study of the Sinha et al. [89]; in particular, is selected to validate the current computational results. The first reason is that Sinha et al. used a short cooling hole in their experiment and included the plenum, which is a real representation of turbine blades. The second reason is that they eliminated the conduction error by using a flat plate made from polystyrene foam with very low thermal conductivity $(0.027 \mathrm{~W} / \mathrm{m} . \mathrm{K})$. This error has not been considered in many of the previous experimental studies which used for validation of the numerical code. The third reason is the uncertainties in the experimental measurements were $( \pm 1.0 \%),( \pm 0.7 \%)$, and $( \pm 0.5 \%)$ for freestream velocity, the total mass flow rate of the jet, and density ratio, accordingly. Therefore, it is generally believed that the film cooling distribution of cylindrical hole in the experimental analysis of Sinha et al. [89] are very accurate and has been extensively used for validation of numerical results [94].

Primary results of the current investigation of the cylindrical micro cooling hole are presented by Abd Alsalam and Jubran [95].

\subsection{Results and Discussion}

This section of the results will be started by the validation of the computational results and comparing them to the experimental data for the case of macro hole, and then the results of micro hole film cooling effectiveness for both centerline and lateral will be presented as well as the flow structure results at various blowing ratios and freestream turbulence intensities. 


\subsubsection{Sensitivity Analysis and Numerical Results Validation.}

The grid independence study is carried out at blowing ratio $M=1.0$ for the case of the macro hole for the centerline effectiveness distribution, as illustrated in Figure 4.3. Four various mesh sizes are generated, which started by a coarser mesh and ended by a very fine mesh with the cells count are varied from $0.49 \times 10^{6}$ to $2.505 \times 10^{6}$ cells, respectively. As Figure 4.3 shows, it can be considered that the mesh sensitivity is established via the fine mesh that has $1.45 \times 10^{6}$ cells whereas a further refinement of the mesh has shown almost no change on the predicted centerline effectiveness. Therefore, fine mesh with $1.45 \times 10^{6}$ cells will be used in the forthcoming analysis to validate the CFD results of the macro hole with the experimental data of [89] and predict both centerline and the lateral effectiveness at low and high blowing ratios. Also, this mesh with $1.45 \times 10^{6}$ cells will be used as the grid sensitivity solution for the discrete round hole case in the following simulations in chapter 6 and chapter 7.

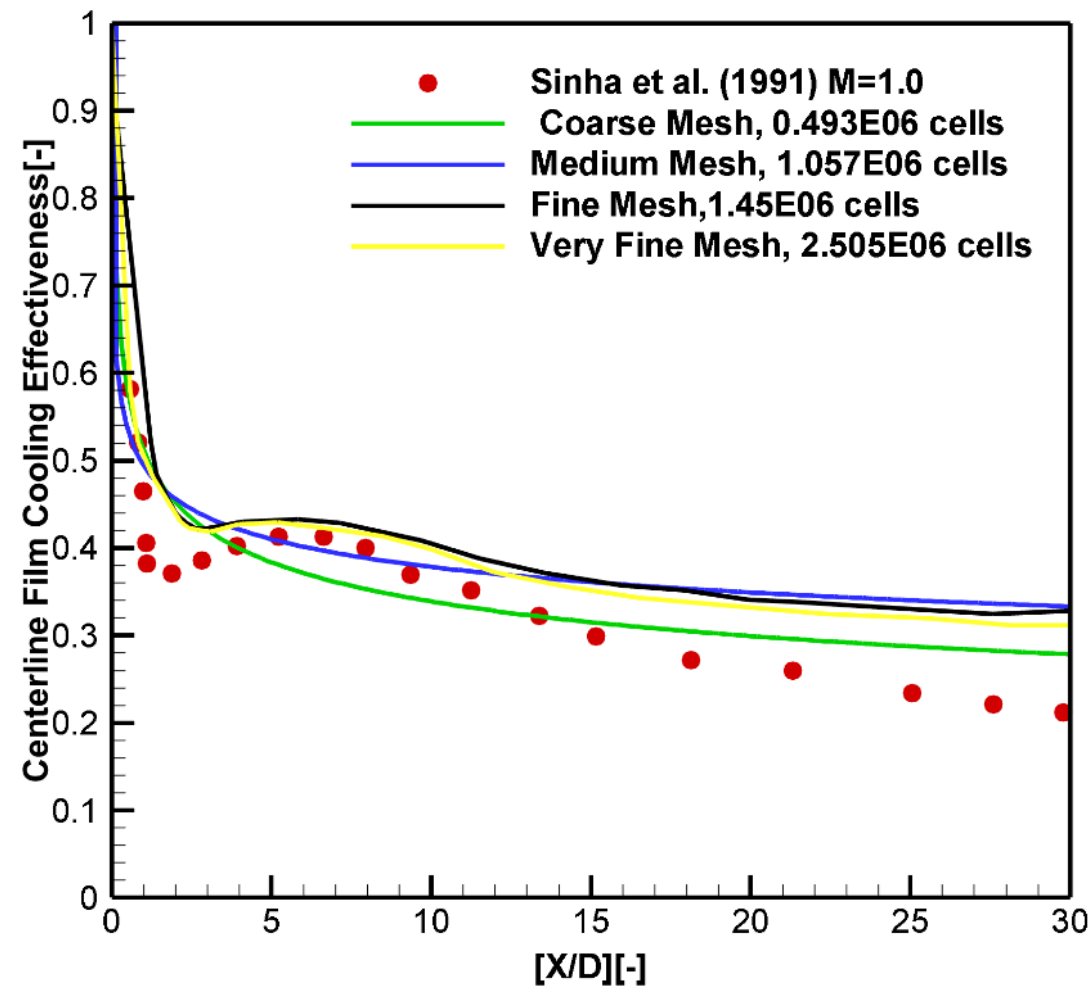

Figure 4.3: The mesh sensitivity for the macro hole shows the centerline film cooling effectiveness at $M=1.0$. 


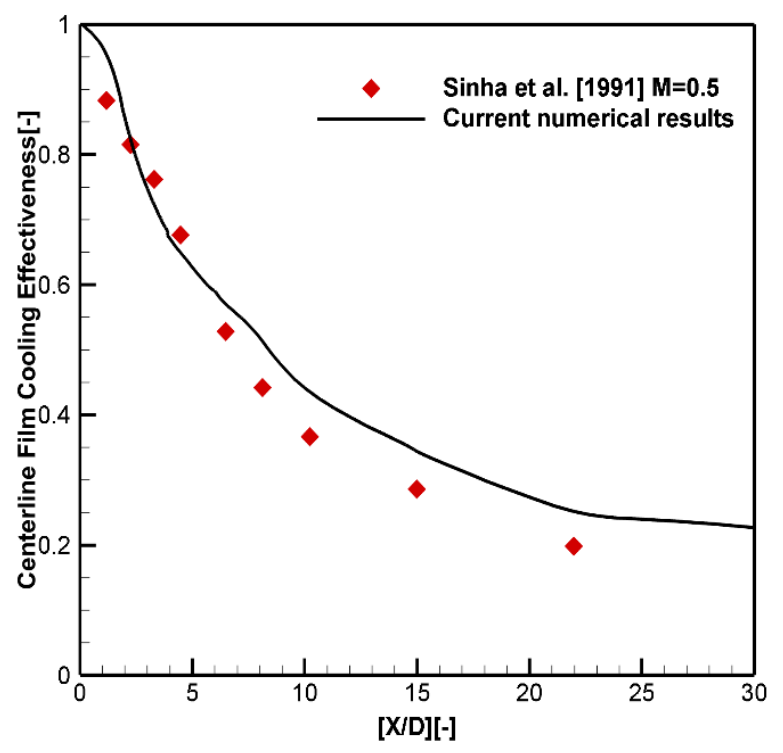

a) The centerline film cooling effectiveness.

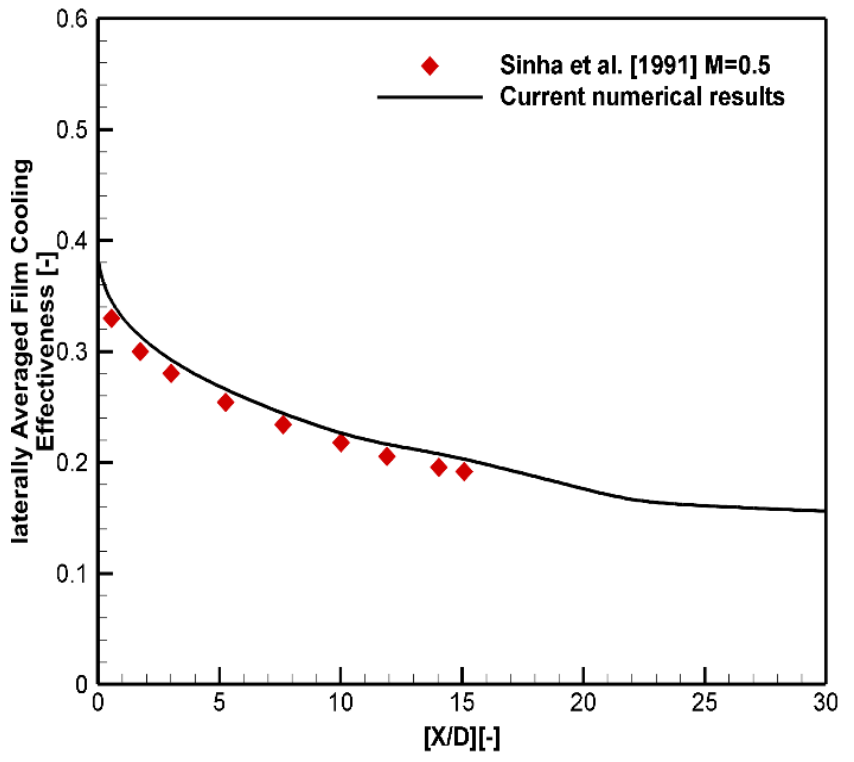

b) The laterally averaged effectiveness.

Figure 4.4: The macro hole centerline and lateral film cooling effectiveness prediction at $M=0.5$ and $D . R=2$ in contrast to the experimental data of [89].

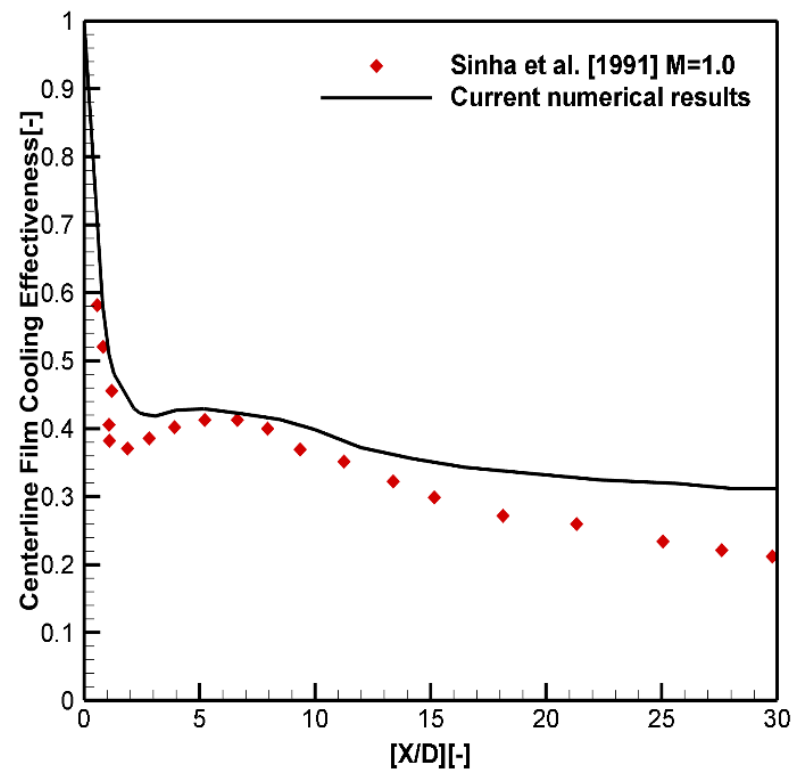

a) The centerline effectiveness.

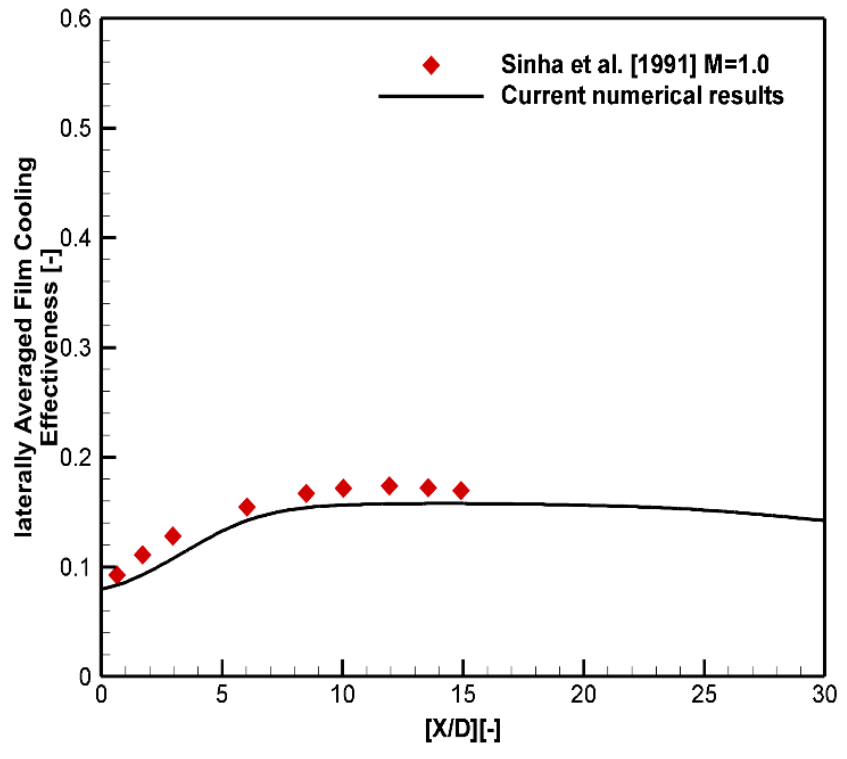

b) The laterally averaged effectiveness.

Figure 4.5: The macro hole centerline and laterally averaged film cooling effectiveness at $M=1.0$ and $D . R=2$ in contrast to the experimental data of [89] 
The macro hole centerline and the laterally averaged effectiveness numerical results at blowing ratio $\mathrm{M}=0.5$ are presented in Figure $4.4 \mathrm{a}$ ) and b), respectively. Both effectiveness results depicted in Figure 4.4 a) and b) show a reasonably good agreement to capture the experimental data, but a slight overprediction of the centerline effectiveness is noticed in the hole vicinity region at $(\mathrm{X} / \mathrm{D}<3)$ and further downstream at $(\mathrm{X} / \mathrm{D}>10)$. The finding of predicted results of film cooling effectiveness at low blowing ratio is similar to the finding of many of the previous numerical studies whereas the cooling performance is well captured when the coolant attached and before the separation occurs. Figure 4.5 a) presents the numerical centerline effectiveness results, while Figure 4.5 b) shows the lateral performance at high blowing ratio $M=1.0$. Even though there is a clear overprediction of centerline effectiveness in the hole vicinity region at $(\mathrm{X} / \mathrm{D}<2)$, the separation and reattachment region at $(2 \leq \mathrm{X} / \mathrm{D} \leq 5)$ is well captured. Further downstream, the overprediction still exists. However, it is not as much intense as immediately downstream the hole exit. This overprediction of the centerline effectiveness distribution at $\mathrm{M}=1.0$ is compensated by a slight underprediction of the spreading of the coolant in the spanwise direction. This typical overprediction of the centerline effectiveness and underprediction of the lateral effectiveness comes as a result of the isotropic feature of the turbulence model; realizable $\mathrm{k}-\varepsilon$ turbulence model, as reported in [96], [97].

Overall, it can be stated that the prediction of the numerical results of the macro hole film cooling effectiveness using the realizable $\mathrm{k}-\varepsilon$ turbulence model is reasonably well captured compared to the experimental data. As such, following the same steps, the mesh sensitivity of the

$200 \mu \mathrm{m}$ diameter discrete cylindrical micro hole is performed and obtained at about $1.39 \times 10^{6}$ cells. The thermal and flow results of the micro hole will be presented and discussed next.

\subsubsection{Thermal and Flow Field Results of Micro Hole at Various Blowing Ratios}

The micro hole centerline and laterally averaged film cooling effectiveness results at varying blowing ratios are depicted in Figure 4.6 and Figure 4.7, respectively. Figure 4.6 shows that for all examined blowing ratios, the centerline effectiveness reaches almost unity immediately at the cooling hole exit. At low blowing ratios; $M=0.25$ and $M=0.5$, the centerline effectiveness is gradually decreased but at high blowing ratios; $M=1.0$ and $M=1.5$, a sharp reduction in the performance are noticed in the hole vicinity region as a result of jet lift-off 


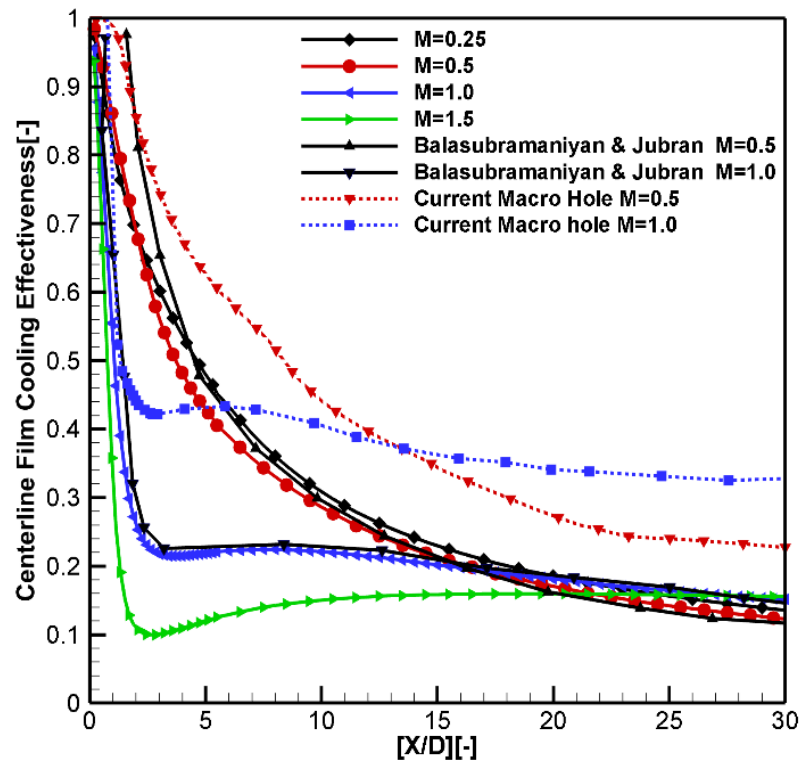

Figure 4.6: The micro hole centerline film cooling effectiveness at $\mathrm{M}=\mathbf{0 . 2 5}-1.5$.

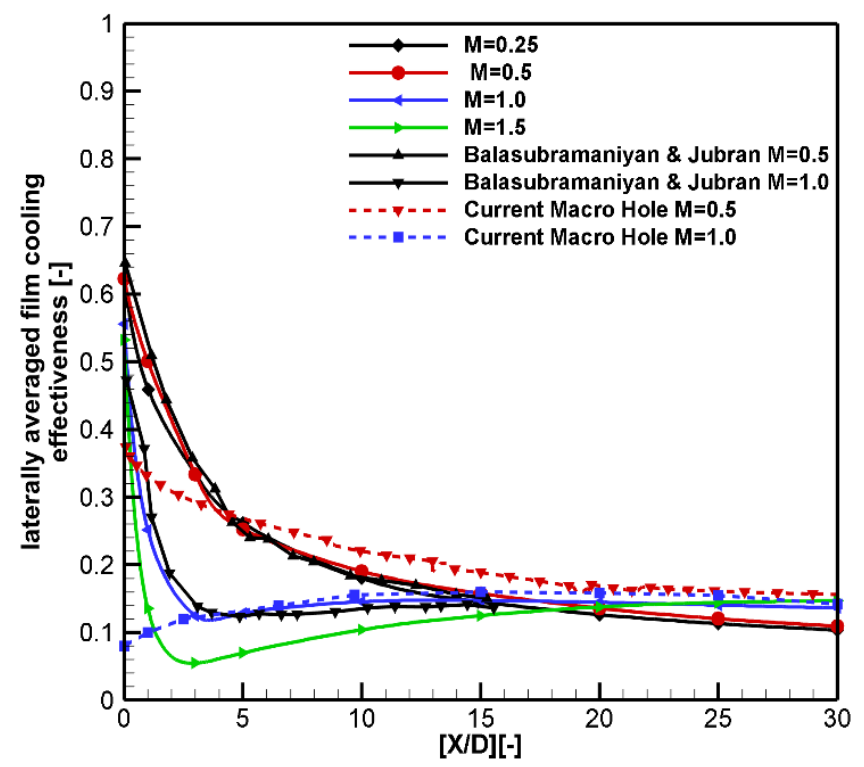

Figure 4.7: The micro hole laterally averaged cooling effectiveness at $M=0.25-1.5$.

phenomenon and the separation of the coolant jet from the plate surface. After that, the micro hole slightly restores its cooling performance due to the reattachment of the coolant jet and shows nearly a plateau trend further downstream. Interestingly, the micro hole at $\mathrm{M}=0.25$ exhibits the best centerline effectiveness, which is approximately $5 \%$ greater than that at $\mathrm{M}=0.5$. This might be interpreted as the micro hole could show promising flexibility in protecting the turbine blades during operation in this range of blowing ratios, low blowing ratio, whilst attaining high engine performance with less coolant consumption. Comparing the centerline effectiveness of micro and macro hole, Figure 4.6 illustrates that directly downstream the cooling hole exit, performance is almost the same for both holes. After that at $(\mathrm{X} / \mathrm{D}>1)$; however, the rate of the reduction of the centerline performance of the micro hole cases is higher than that for the macro hole specifically at high blowing ratio. This may be due to the rapid mixing and penetration of the coolant into the freestream that leads to reduce the micro jet ability to maintain its structure.

Comparing the micro hole cooling performance of the current study to the previous numerical results of Balasubramaniyan and Jubran [23], there is a good agreement between both results at blowing ratios $\mathrm{M}=0.5$ and 1.0 in the far downstream. But in the hole vicinity region, a slight divergence is noticed whereas Balasubramaniyan and Jubran [23], have stated that the micro hole performs somewhat better than that for the macro hole at $(\mathrm{X} / \mathrm{D} \leq 1.0)$, which is not the case in the 
current study. This might be due to the difference in the applied turbulence boundary conditions that are resulting in a different prediction of flow field results in the hole vicinity region.

The micro hole laterally averaged film cooling effectiveness predicted results at various blowing ratio are shown in Fig. 4.7. It can be seen from the plot that for all examined blowing ratios micro hole laterally averaged effectiveness is better than that of the macro hole in the adjacent region downstream hole at $(\mathrm{X} / \mathrm{D} \leq 5.0)$, however, it shows almost the same performance far downstream at high blowing ratios. It can also observe that spreading of the coolant jet in the lateral direction at low blowing ratios is wider in the hole adjacent downstream region up to $(\mathrm{X} / \mathrm{D} \leq 5.0)$. But in the far downstream area, the high blowing ratios show slightly better diffusion of the coolant jet. The Blowing ratio $\mathrm{M}=0.5$ gives the optimal laterally averaged effectiveness at $(\mathrm{X} / \mathrm{D} \leq 5.0)$ whilst the blowing ratio $\mathrm{M}=1.5$ yields the best performance in the far downstream area. Hassan [21] stated that micro jet has its unique feature

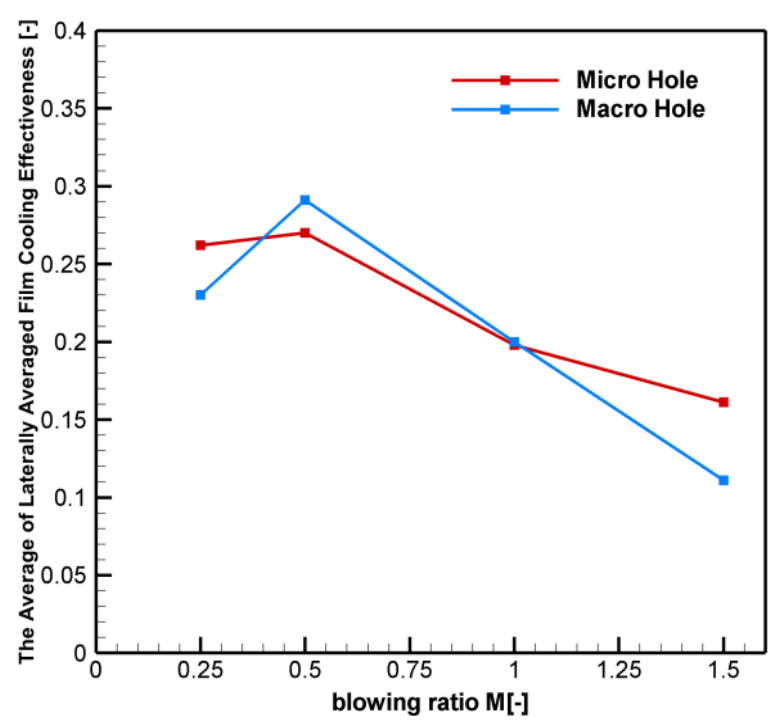

Figure 4.8: The comparison of the averaged laterally averaged film cooling effectiveness between macro and micro hole.

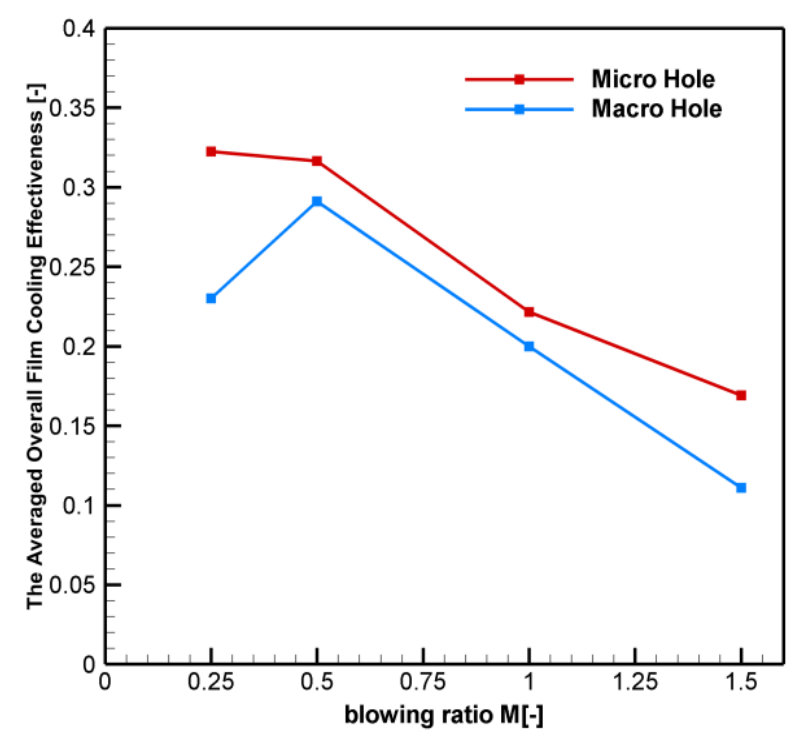

Figure 4.9: Comparison of the average overall film cooling effectiveness between macro and micro hole.

which appears in less turbulent enhancement and penetration into the mainstream, and it is believed that this is the reason for the good performance of the micro hole at blowing ratio $\mathrm{M}=0.5$. Moreover, the second reason could be due to the 3D flow structure of the micro jet that shown a reduction in the CRVP strength and size, which resulted in a better local lateral performance at low and high blowing ratios $(\mathrm{M}=0.5$ and 1.0) as reported by Balasubramaniyan and Jubran [23]. 
A comparison of the averaged laterally averaged effectiveness at various blowing ratios between the macro and the micro hole is shown in Figure 4.8. Interestingly, Figure 4.8 exhibits that the micro hole in most of the examined blowing ratio cases provided better averaged laterally averaged effectiveness as a result of the enhancement in the lateral coolant spreading and coolant uniformity. On the other hand, it shows a slightly lower performance at $M=0.5$, which could be attributed to the fact that coolant jet is not able to sustain its structure due to mixing and thus caused a reduction in the cooling performance. Moreover, it can be observed in Fig. 4.8 that the micro hole has achieved good averaged laterally averaged effectiveness with a $12 \%$ reduction in the coolant blowing ratio at the range of the highest averaged lateral film cooling effectiveness. This reduction of the coolant blowing ratio is an indication of saving in coolant flow consumptions.

A comparison of the overall averaged film cooling effectiveness for micro and macro holes at different blowing ratios is illustrated in Fig. (4.9). It can be seen from the plot that the micro hole offers higher overall averaged film cooling effectiveness contrary to that of the macro hole, and the overall increase is about $30 \%$. This progression in the micro hole film cooling performance increases the possibility of applying micro film cooling technology as an effective method to cool turbine blades.

The effect of the micro hole film cooling technique on the surrounding flow field will be given in this section by visualizing the velocity contours and flow structure results. The velocity contours results, depicted in Figure 4.10, are presented at the midplane $\mathrm{z}=0$ at blowing ratios $\mathrm{M}=$ $0.25-1.5$, while the emphasis will be primarily in the hole vicinity region. Figure 4.10 a) and b) demonstrates that the velocity contours at a low blowing ratios, $\mathrm{M}=0.25$ and $\mathrm{M}=0.5$, have a highly skewed jet shown inside the cooling hole, and then it turns toward the hole leading edge resulting in a low jet momentum at the cooling hole trailing edge. Subsequently, at the time coolant and the freestream meet and interact, the freestream will force the coolant to remain attached and parallel to the plate surface since it has a high momentum. For this reason, the micro hole has shown a good cooling performance at low blowing ratios $\mathrm{M}=0.25$ and 0.5 . The velocity contours demonstrate a broader reverse region directly downstream of the hole as well as a high jet momentum at the hole leading edge for the case of blowing ratio $M=1.0$, as shown in Fig. 4.10 c). Hence, the jet lifted off from the plate surface and caused a reduction in the centerline and laterally averaged film cooling effectiveness. At a blowing ratio of $\mathrm{M}=1.5$ Fig. $4.10 \mathrm{~d}$ ) confirms 
that the micro jet has a very high coolant momentum inside the cooling hole, which causes the jet to lift-off from the surface. Accordingly, micro hole cooling effectiveness sharply drops in the hole adjacent region at $(1 \leq \mathrm{X} / \mathrm{D} \leq 4)$, as depicted early in Figure 4.6. Overall, the analysis of micro hole velocity flow field results at various blowing ratios revealed a comparable trend to that of the macro hole whereas the coolant jet either remains attached, lifted off, or reattached to the plate surface based on the blowing ratio which was reported in many previous CFD and experimental studies [89],[94],[96], [98], and [99].
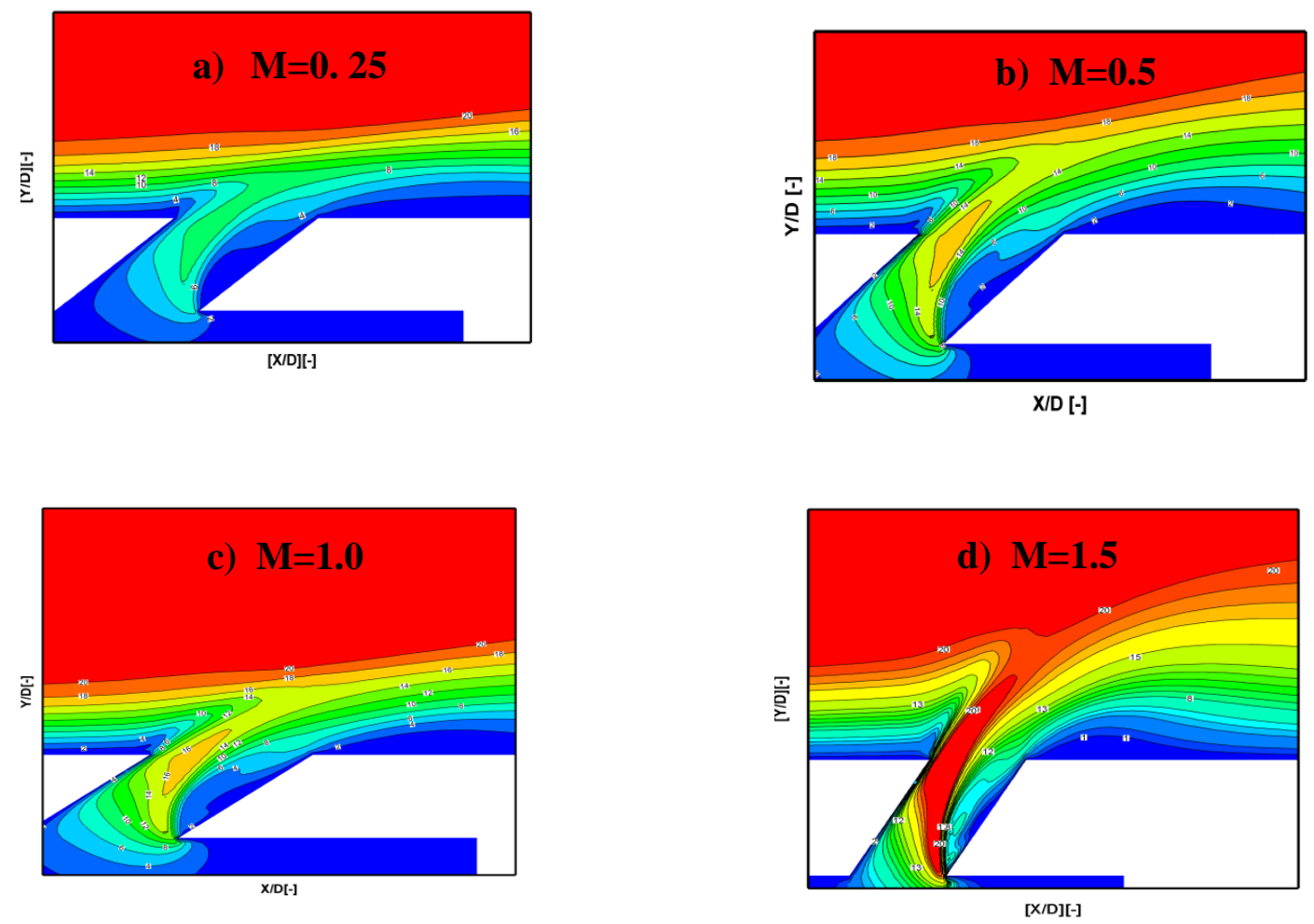

Velocity contour [m/sec] \begin{tabular}{llllllllllllllll|}
\hline & 2 & 4 & 5 & 6 & 8 & 9 & 10 & 11 & 12 & 13 & 15 & 17 & 18 & 19 & 20 \\
\hline
\end{tabular}

Figure 4.10: The velocity contours at midplane $Z=0$ at blowing ratio $M=0.25-1.5$.

The flow structure in film cooling produced by the interaction of the freestream and the coolant has a significant effect on the cooling performance, and it is primarily shown in the pair of counter-rotating vortices (CRVP). Therefore, the micro hole flow structure will be assessed herein at different blowing ratios, as shown in Figure 4.11. The assessment of the flow structure is carried out first between the macro and micro holes at blowing ratio $\mathrm{M}=0.5$ at four downstream locations; $\mathrm{X} / \mathrm{D}=0,1,3$, and 5 , and then only micro hole flow structure results will be presented at $\mathrm{M}=0.25,1.0$, and 1.5 . The flow structure results will be given in the $\mathrm{YZ}$ plane perpendicular to 
the plate surface using the velocity vectors and temperature contours. The velocity vectors will indicate the vortices strength and size, while the temperature contours will express the mixing rate of the coolant with the mainstream. Figure $4.11 \mathrm{a}$ ) and b) exhibits the flow structure results at $\mathrm{M}=0.5$ for macro and micro holes, and it can be observed that the coolant core is significantly attached to the plate surface for both holes at the downstream locations of $(X / D=0$ and 1$)$. However, the core attachment region of the micro hole is much wider than that of the macro hole whereas the coolant core at $(\mathrm{X} / \mathrm{D}=0)$ covers the lateral domain of $( \pm 0.4)$ and $( \pm 0.5)$ for the macro and micro holes, respectively, while at $(X / D=1)$ it covers the domain of $( \pm 0.15)$ and $( \pm 0.3)$, for the macro and micro holes, respectively. The vertical distribution of the coolant from the micro and macro hole is almost identical. Moreover, the mixing of coolant and freestream from the micro hole is less than that of the macro hole, and the coolant core is more competed for the macro hole, as depicted by temperature contours. In terms of the vortices structure, Figure 4.11 a) illustrates the generation of a bit stronger counter-rotating vorticity pair structure in the case of the macro hole, contrary to that of the micro hole, which attempts to lift the coolant away from the surface and turns the freestream flow toward it. Instead, the micro hole has waker and less defined vortices structure, which enhances the coolant spreading in the spanwise direction and reduces the mixing of coolant with the mainstream, as shown in Figure $4.11 \mathrm{~b}$ ). Moving further downstream, at $(X / D=3$ and 5), the coolant core is still attached for the macro and micro hole at those two positions, but the micro hole still shows a wider coolant distribution spanwisely whereas at $(X / D=3)$ the coolant covers the lateral region of $( \pm 0.135)$ for the macro hole, and it covers the area of $( \pm 0.28)$ for the micro hole, while at $(X / D=5)$ the coolant covers the region of $( \pm 0.12)$ and $( \pm 0.25)$ for the macro and micro holes, respectively. Also, because of the devolvement of the CRVP and its stronger structure, it enhances the mixing, and it reduces the coolant lateral distribute compared to that of the micro hole. On the other hand, the swirling flow starts to be less notable from the micro hole, and the CRVP is very weak. This flow structure attained from the micro hole at $M=0.5$ explains the high lateral effectiveness results shown previously in Fig. 4.7, which resulted from better coolant spanwise coverage. The flow structure for the lowest blowing ratio case of the micro hole, $M=0.25$, shown in Figure $4.11 \mathrm{c}$ ) displays the coolant jet is attached to the plate 
a) Macro hole $\mathrm{M}=0.5$
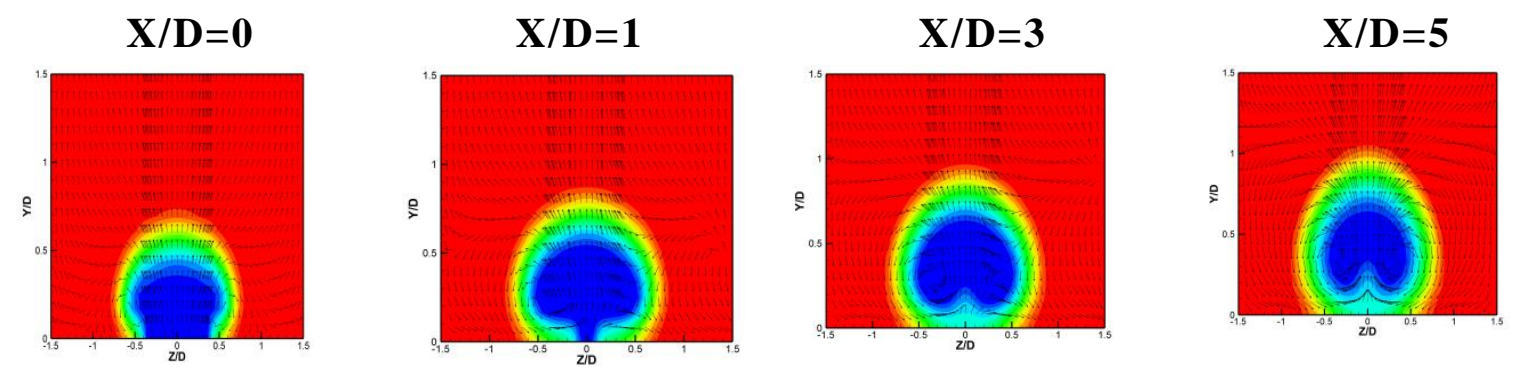

b) Micro hole $\mathrm{M}=0.5$
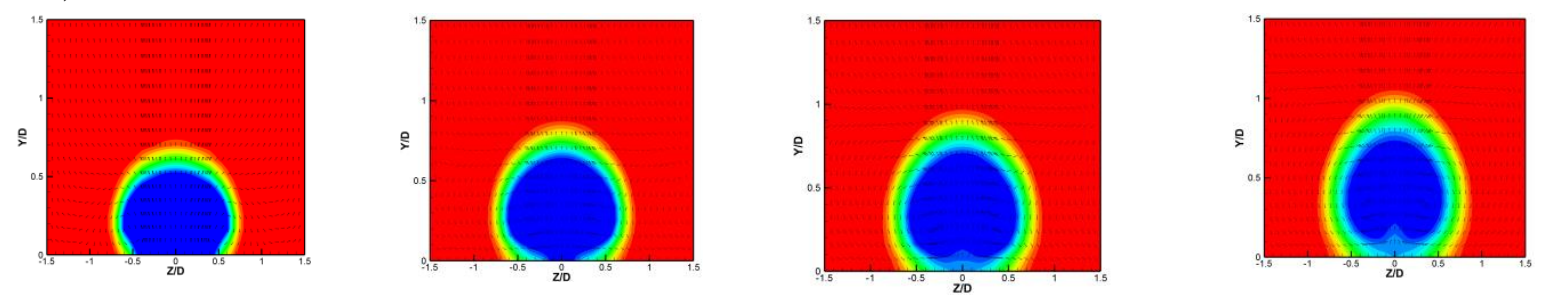

c) Micro hole $\mathrm{M}=0.25$
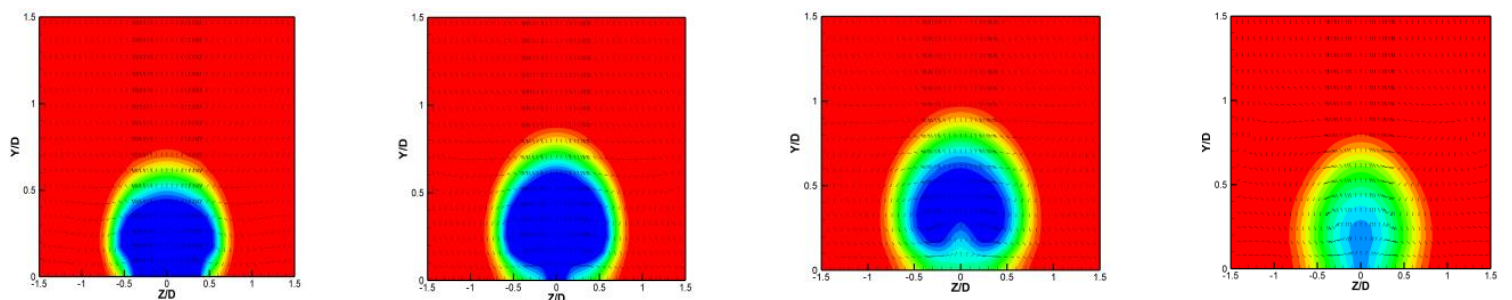

d) Micro hole $\mathrm{M}=1.0$
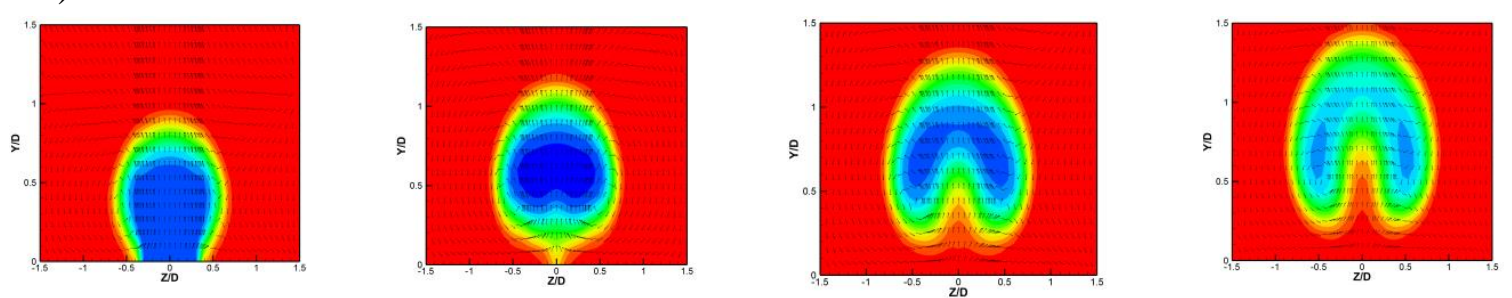

e) Micro hole $\mathrm{M}=1.5$
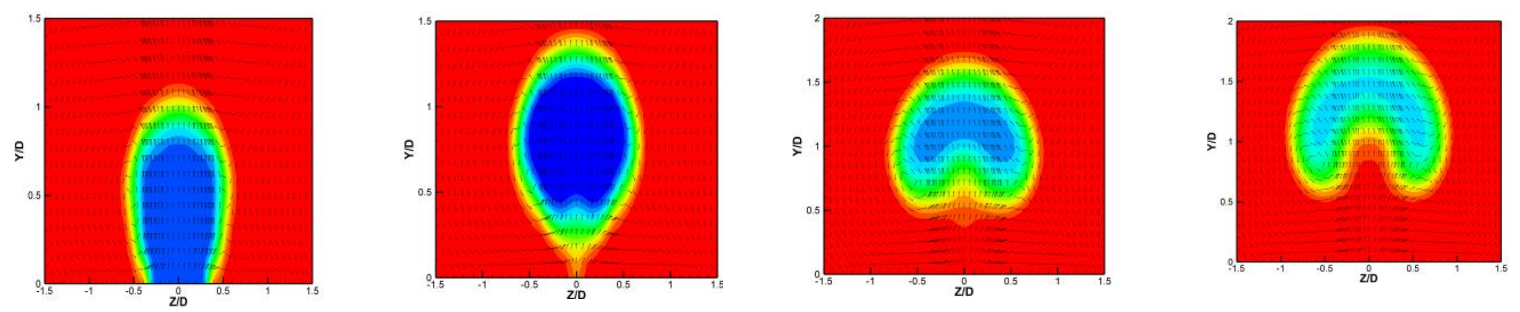

Temperature [K] 150160170180190200210220230240250260270280290300

Figure 4.11: The flow structure results at four different locations downstream of the cooling hole at $X / D=0,1,3$, and 5 at various blowing rate $M=0.25-1.5$. 
surface in the hole vicinity area at $(\mathrm{X} / \mathrm{D}=0$ and 1$)$, while the coolant lateral coverage is slightly less than that of the micro hole at $M=0.5$. Further downstream at $(X / D=3$ and 5$)$, the coolant jet could not hold its structure, and it gets mixed rapidly with the freestream as a result of low coolant jet momentum while a less define CRVP has appeared. The CRVP adverse effect is more distinct on the micro hole cooling performance in the hole adjacent area at high blowing ratios, as shown in the case of $M=1.0$ and 1.5, Figure $4.11 \mathrm{~d}$ ) and e). In those two cases, the coolant core is shown only to be attached to the surface at $(X / D=0)$ while the coolant has a high jet momentum that causes the jet to lift-off from the plate surface while intense mixing occurs. As such, micro hole cooling performance is degraded in the downstream region $(1<\mathrm{X} / \mathrm{D}<5)$ at high blowing ratios.

\subsubsection{Thermal performance of Micro Hole at Various Freestream Turbulence Intensity}

Mainstream turbulence intensity is one of the operating conditions that strongly affect the film cooling performance. In real engine operating conditions, the flow upstream the turbine is typically presented by a high level of freestream turbulence intensity (FSTI) that might be beyond $20 \%$, which changes the mixing and penetration of the coolant jet with freestream [100]. As such, this section assesses the influence of the freestream turbulence intensity (FSTI) on centerline effectiveness, laterally averaged effectiveness, and the local effectiveness of micro hole at two blowing ratios; $\mathrm{M}=0.5$ and $\mathrm{M}=1.0$. The numerical results of the centerline film cooling effectiveness at various freestream turbulence intensities (FSTI $=0.2 \%, 1 \%, 5 \%, 10 \%$, and 20\%) at $M=0.5$ and $M=1.0$ are depicted in Figure 4.12 a) and b), respectively. Ultimately, Figure 4.12 a) and b) demonstrate a similar trend for centerline effectiveness discussed early in Figure 4.6, however, the increase in the (FSTI) leads to a diminutive decrease in the performance. At M=0.5, Figure 4.12 a) exhibits that increasing (FSTI) from $0.2 \%$ to $20 \%$ has a negligible effect on reducing the centerline effectiveness which is, in general, does not exceed $\approx 6 \%$. This degradation of the centerline film cooling performance by elevating FSTI comes as a result of enhancing mixing, and it seems that the coolant jet is totally dissipated after downstream distance $(\mathrm{X} / \mathrm{D} \geq 20)$. Figure $4.12 \mathrm{~b}$ ) exhibits the centerline effectiveness at a high blowing ratio $\mathrm{M}=1.0$. It can be observed from this plot that by increasing the freestream 


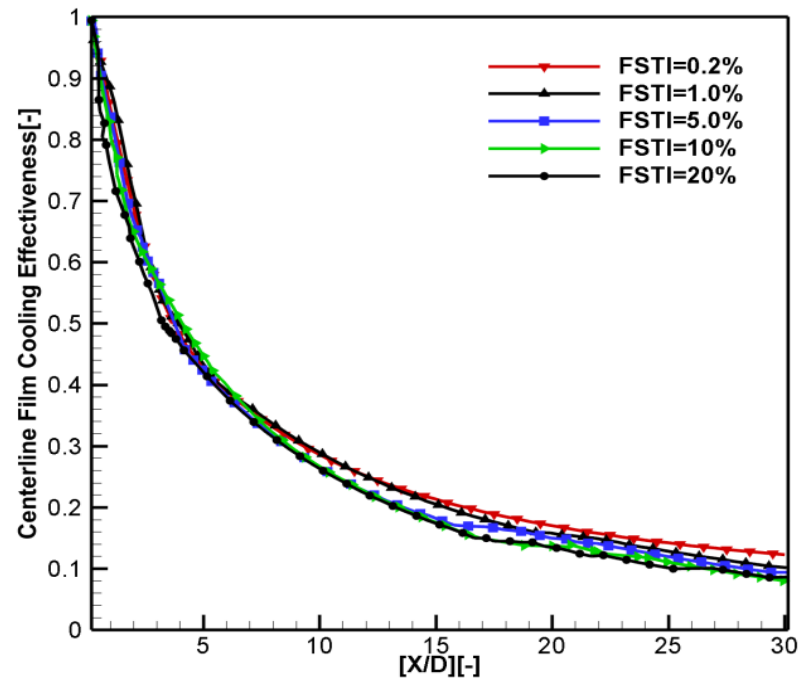

a) Centerline effectiveness at $\mathrm{M}=0.5$.

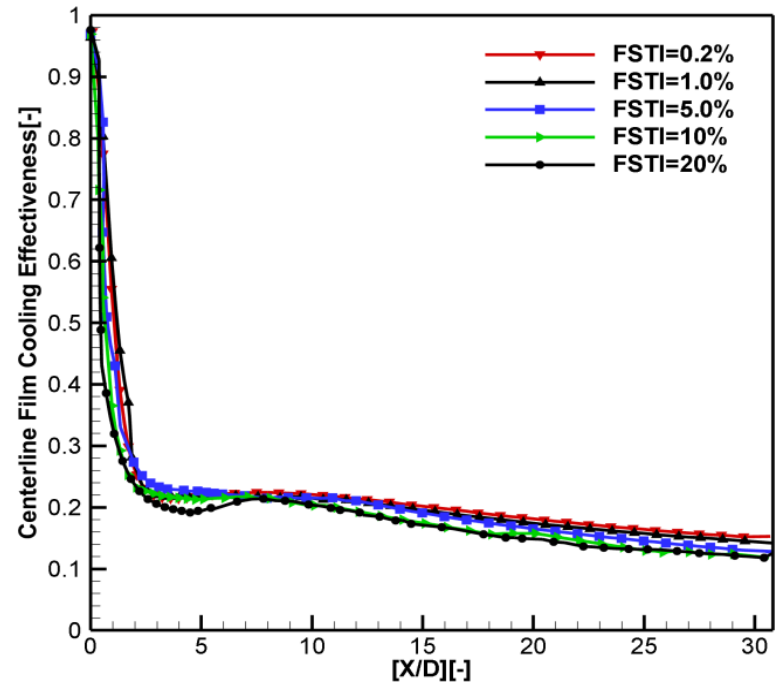

b) Centerline effectiveness at $\mathrm{M}=1.0$.

Figure 4.12: The micro hole centerline film cooling effectiveness at various freestream turbulence intensity at $M=0.5$ and $M=1.0$.

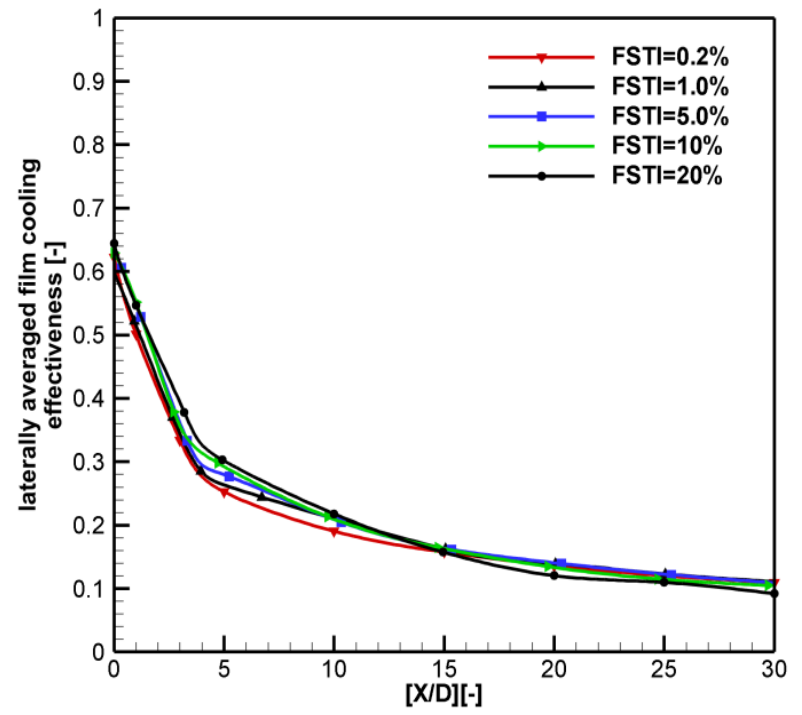

a)Laterally averaged effectiveness at

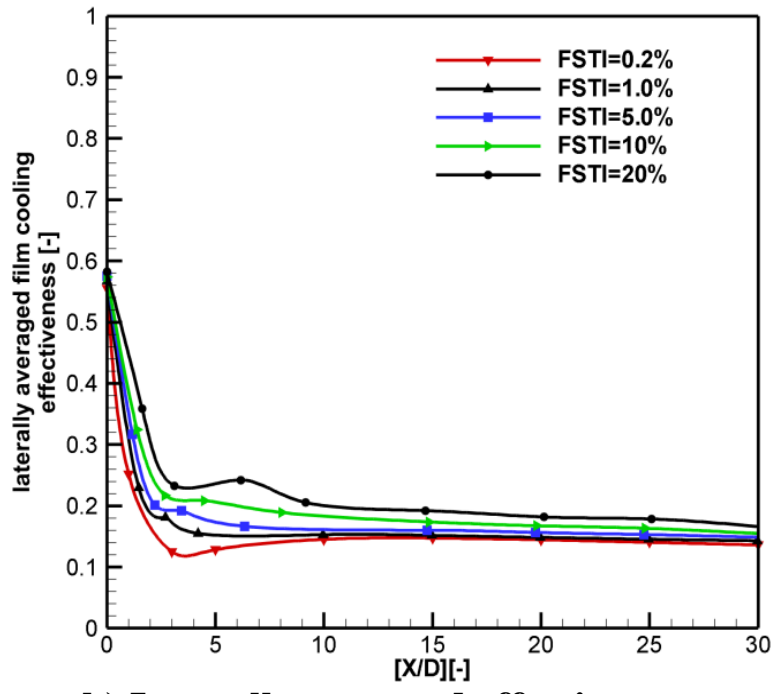

b) Laterally averaged effectiveness at $\mathrm{M}=1.0$.

Figure 4.13: The micro hole laterally averaged film cooling effectiveness at various freestream turbulence intensity at $M=0.5$ and $M=1.0$.

turbulence intensity; (1) a continuation in the decay of the centerline effectiveness in the hole adjacent region and far downstream, (2) the separation and the reattachment region is being slightly shifted downstream in most of the cases as a result of the intensive mixing between the coolant and the freestream at this high blowing ratio. 
The laterally averaged effectiveness results of the micro hole at different freestream turbulence intensities at blowing ratios $M=0.5$ and $M=1.0$ are shown in Figure 4.12 a) and b), respectively. At low blowing ratio, the lateral effectiveness performance shown in Fig. 4.12 a) is almost the same for all examined (FSTI), while there is a minor change that appeared within the region of $(5 \leq X / D \leq 15)$. But, at a high blowing ratio, increasing the FSTI looks to change the lateral cooling performance significantly, as shown in Fig. 4.12 b), whereas the effectiveness is improved for the entire downstream region. Moreover, the plot shows that increasing the FSTI has affected the lateral coolant distribution within the range of $(2 \leq X / D \leq 10)$ in which a better lateral spreading is noticed whereby the effectiveness curve is shifted upstream compared to lowest FSTI case. The best lateral performance is obtained at high blowing ratio $\mathrm{M}=1.0$ at FSTI $=20 \%$, which is $\approx 15 \%$ higher than that at FSTI $=0.2 \%$. A likely cause for this increase in micro hole lateral cooling performance at high blowing ratio is that the coolant has high momentum and the increase in turbulence intensity caused an increase in the mixing rate between the coolant jet and the freestream, which in turn helps to distribute the coolant jet more widely in the spanwise direction.

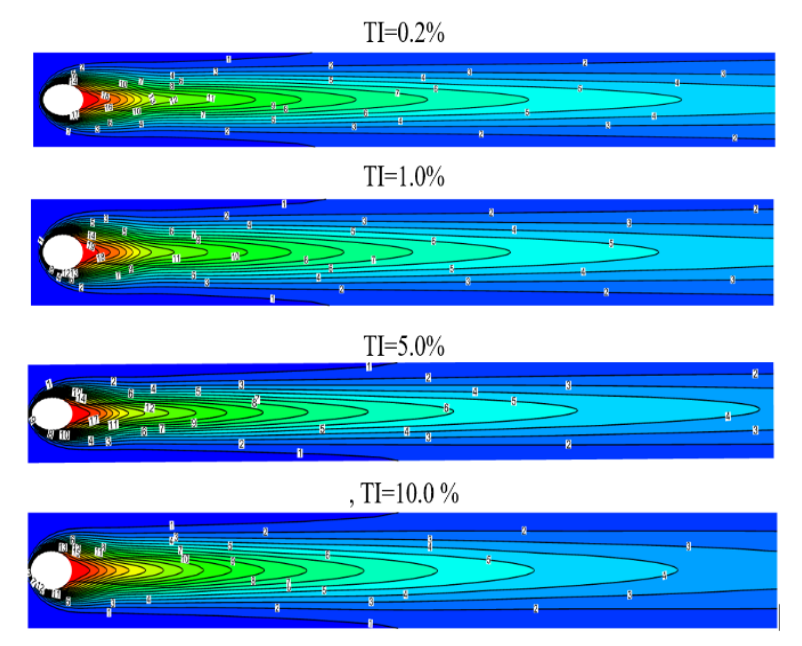

$\mathrm{TI}=20.0 \%$

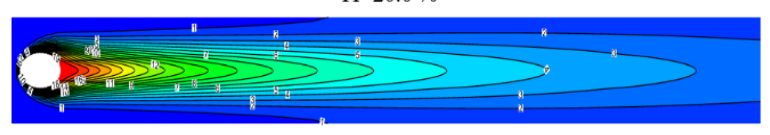

a) $\mathrm{M}=0.5$

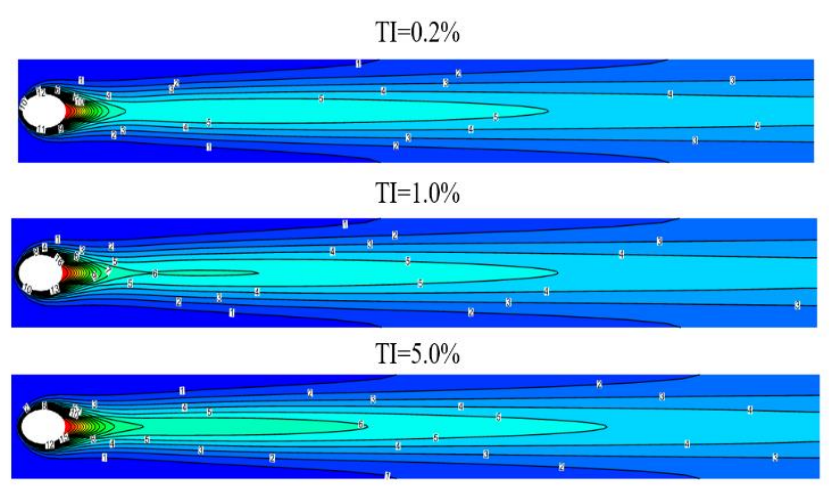

$\mathrm{TI}=10.0 \%$

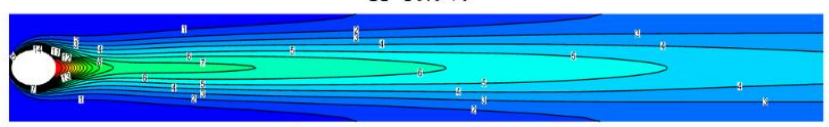

$\mathrm{TI}=20.0 \%$

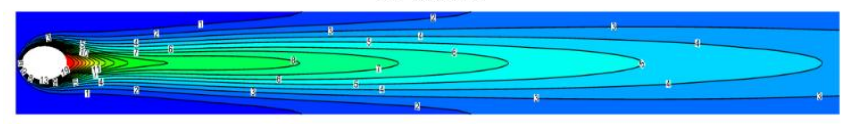

b) $\mathrm{M}=1.0$

$\left.\begin{array}{ccccccccccccccccccc}1 & 2 & 3 & 4 & 5 & 6 & 7 & 8 & 9 & 10 & 11 & 12 & 13 & 14 & 15 & 16 & 17 & 18 & 19 \\ 0.05 & 0.1 & 0.15 & 0.2 & 0.25 & 0.3 & 0.35 & 0.4 & 0.45 & 0.5 & 0.55 & 0.6 & 0.65 & 0.7 & 0.75 & 0.8 & 0.85 & 0.9 & 0.95\end{array}\right]^{2} \times$

Figure 4.14: The local film cooling effectiveness contours at the plate surface at various (FSTI) at blowing ratio $\mathrm{M}=\mathbf{0 . 5}$ and $\mathrm{M}=\mathbf{1 . 0}$. 
Figure 4.14 illustrates a comparison of a 2-D contour plot of the micro hole local film cooling effectiveness at the plate surface at two blowing ratios and various freestream turbulence intensity. The plot displays that the distribution of the local effectiveness for both blowing ratios is symmetry about the centerline $\mathrm{Z}=0$. It is obvious from the plot that the zone of the high local film cooling effectiveness appears in the adjacent region of the hole exit for both blowing ratios at all examined levels of (FSTI), while the optimum coverage mostly appears at a blowing ratio of $\mathrm{M}=0.5$ and $\mathrm{FSTI}=20 \%$. Also, Figure 4.14 a) shows that at $\mathrm{M}=0.5$ there is a spanwise coolant coverage improvement up to a downstream distance $(\mathrm{X} / \mathrm{D} \leq 15)$ when increasing the FSTI, but further downstream it illustrates a slight reduction in performance as a result of coolant mixing and dispersion of coolant into the freestream. Figure 4.14) b) exhibits that at high blowing ratio $\mathrm{M}=1.0$, lower performance is attained compared to that at $\mathrm{M}=0.5$ while a wider zone of coolant distribution spanwisely occurs far downstream. This reflects the better lateral performance of the micro hole at high blowing ratios in the far downstream region, whereas the coolant coverage improves by increasing the FSTI. The wide spreading of the coolant in the spanwise direction is attributed to the reattachment of the coolant jet far downstream of the hole exit at high blowing ratio.

\subsection{Conclusions}

In this chapter, the film cooling performance and flow field of a discrete cylindrical micro hole with a $200 \mu \mathrm{m}$ diameter over a flat plate have presented at different coolant to freestream operating conditions. It is found that the best centerline and lateral cooling performance of the micro hole are obtained at low blowing ratios. Moreover, it is revealed that micro hole offers about a $30 \%$ increase in the overall film cooling effectiveness while there is about a $12 \%$ reduction in the coolant blowing ratio within the range of the highest averaged lateral effectiveness; which means a saving in coolant consumption. The flow field results show the existence of the CRVP; however, at a smaller size and less strength of the vorticial structure, which assists the coolant spreading more widely in the spanwise direction. At a high blowing ratio, increasing the freestream turbulence intensity to match the engine like operating conditions slightly reduced the centerline effectiveness and caused an increase in the laterally averaged effectiveness. Finally, the micro cooling hole offers a considerable improvement in the overall film cooling performance and has the potential to effectively prevent the thermal failure of 
turbine blade while maintaining its structure and material strength. As such, this micro cooling technique could lead to more efficient and environmentally friendly aero- engines. 


\section{CHAPTER FIVE: PREDICTION OF FILM COOLING PERFORMANCE OF UPSTREAM SISTER HOLES USING DIFFERENT TURBULENCE MODELS}

Numerous computational and experimental investigations have been conducted to develop new film cooling hole shapes and arrangements to improve cooling performance at high blowing ratios by eliminating the jet lift-off. Sister holes is one of film cooling techniques that involves certain hole arrangements, and were introduced almost decade ago and has revealed a notable improvement in the thermal performance over a wide range of blowing ratios as well as a dramatic reduction on the strength of the counter-rotating vortices of the primary hole and maintained the coolant jet attached to the plate surface. The investigation reported in this chapter is different from all of the previous research available in open literature on sister holes film cooling, in such that a numerical prediction of the upstream sister holes thermal performance is carried out based on the only one experimental data avalibe on sister holes in the study of Wu et al. [10]. The investigation is performed at low and high blowing ratios to examine the ability of several turbulence models and near-wall treatments to predict the upstream sister holes lateral performance. From this chapter and onwards, the focus of the thesis will be on sister holes film cooling numerical prediction of their cooling performance.

\subsection{Problem Statement}

To the best of our knowledge, numerical prediction of the sister holes film cooling performance using various turbulence models has not been documented yet. As such, in this chapter, a CFD investigation is performed to evaluate the prediction capability of five turbulence models available on ANSYS-Fluent, specifically: realizable k-epsilon, standard k-epsilon, RNG k-epsilon, Reynolds stress model, and Spalart-Allmaras model combined with enhanced wall treatment to predict the film cooling effectiveness of the upstream sister holes in comparison to the only experiment study available on sister holes that was carried out by Wu et al. [10] at low and high blowing ratios. Moreover, the effect of the near-wall modeling on the prediction of film cooling performance is studied using various near-wall treatments along with realizable k-epsilon at low blowing ratio. The recent experimental work of $\mathrm{Wu}$ et al. [10] is the benchmark study for the current research with the emphasis on the upstream sister holes case. 


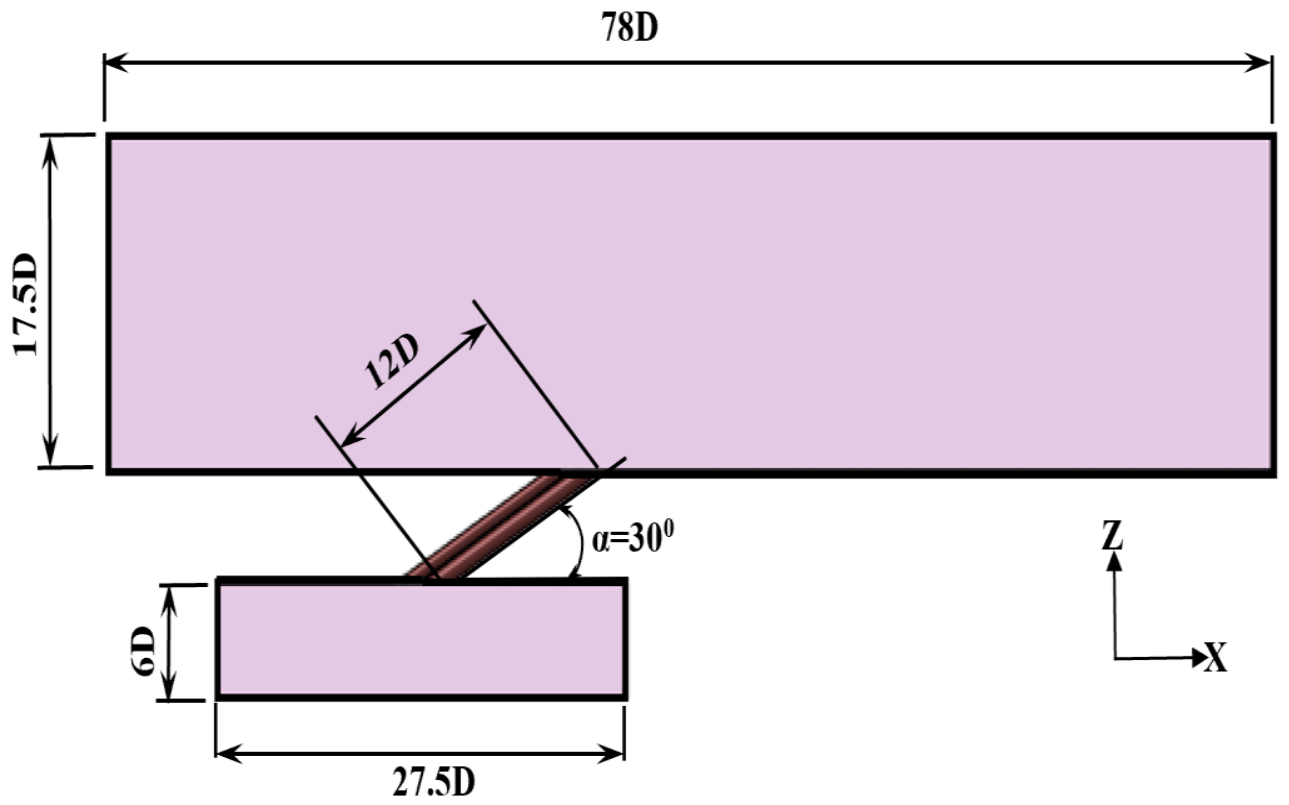

a) Side View

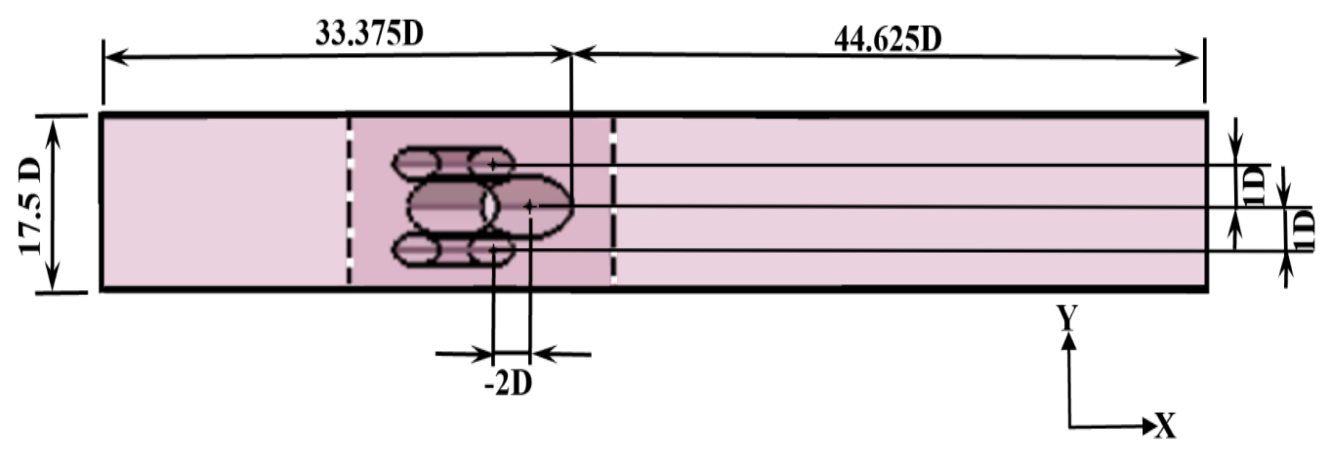

b) Top view

Figure 5.1: The 3D model of the upstream sister holes a) side view and b) top view. (Not to scale).

To replicate their model, a 3D computational model is built, which consists of a primary cooling hole with a diameter of $(\mathrm{D}=4 \mathrm{~mm})$ that is used as a reference scale to describe the main model parts. The primary cooling hole has the length to diameter ratio (L/D) $=12$ and injected to the streamwise at $\left(\alpha=30^{\circ}\right)$. As reported in the experiment [10], the model comprises three main parts, specifically : part A; mainstream channel, part B; cooling holes, and part C; the plenum. The mainstream channel has a dimension of $(78 \mathrm{D}, 17.5 \mathrm{D}$, and $17.5 \mathrm{D}$,$) in (\mathrm{X}, \mathrm{Y}$, and $\mathrm{Z})$ directions, respectively. The sister holes have a diameter of $0.75 \mathrm{D}$ and are located (-2D) upstream the primary hole with the same (L/D) ratio and the injection angle of the main hole. The cooling air plenum has a dimension of $(27.5 \mathrm{D}, 17.5 \mathrm{D}$, and $6 \mathrm{D})$ in $(\mathrm{X}, \mathrm{Y}$, and $\mathrm{Z})$ directions, respectively. The 
point of origin is placed at the main hole trailing edge whereas the computational domain is extended 33.37D upstream it in X-axis. To reduce the unnecessary complication of the 3D model and the computational intensity, the coolant flow is assumed to be injected from the plenum lower surface while in the experiment the coolant jet was injected from a pipe attached to the side face of the plenum that is parallel to the positive $\mathrm{X}$-axis. Figure $5.1 \mathrm{a}$ ) and b) shows a 3D computational model of the upstream sister holes side and top views, respectively.

\subsection{Simulation parameters and Computational Overview}

The applied boundary conditions in this section are identical to the experimental data of $\mathrm{Wu}$ et al. [10]. The computational analysis is performed at Reynolds number of $\operatorname{Re}_{D}=3400$, which is based on the mainstream velocity and primary hole diameter. Moreover, the mainstream temperature is $323.15 \mathrm{~K}$ while the coolant temperature is $306.15 \mathrm{~K}$, which maintained a density ratio of (D.R=1.05). Table 5.1 shows the freestream and coolant boundary conditions, as stated in the experimental measurement of $\mathrm{Wu}$ et al. [10].

Table 5.1: The mainstream and coolant flow parameters [10].

\begin{tabular}{|c|c|}
\hline \hline Property & Value/Units \\
\hline Freestream Temperature & $323.15 \mathrm{~K}$ \\
Coolant Temperature & $306.15 \mathrm{~K}$ \\
Freestream Velocity & $15.28(\mathrm{~m} / \mathrm{sec})$ \\
Density Ratio (D.R) & 1.05 \\
Freestream Turbulence Intensity & $5.0 \%$ \\
Coolant Turbulence Intensity & $1.0 \%$ \\
M & $0.3-2.5$ \\
Operating Pressure & $101325(\mathrm{~Pa})$ \\
\hline
\end{tabular}

To get a precise numerical solution, it is essential in the simulation to define the turbulence parameters such as the turbulence intensity. The turbulence intensity of the freestream is set to FSTI $=5 \%$. However, as a result of lack of detailed empirical data of the coolant jet during the experimental setup of $\mathrm{Wu}$ et al. [10], the turbulence intensity of coolant is assumed a low value $\approx 1 \%$, as shown in Table 5.1. The second turbulence parameter is the turbulent length scale $\left(\mathrm{L}_{\mathrm{t}}\right)$ for the freestream inlet and plenum inlet, which can be calculated as $\left(\left(\mathrm{L}_{\mathrm{t}}\right)_{\infty}=0.05 \mathrm{~h}\right)$ and $\left(\left(\mathrm{L}_{\mathrm{t}}\right)_{\mathrm{c}}\right.$ $=0.07 \mathrm{Dh}$ ), respectively, whereas $(\mathrm{h})$ is the height of the mainstream channel, and $\mathrm{D}_{\mathrm{h}}$ is the hydraulic diameter of the coolant inlet section [81], [85].The cooling performance of the upstream 
sister holes are examined at six blowing ratios $\mathrm{M}=0.3-2.5$, whereas the blowing ratio can be determined by $\left(M=D \cdot R * V_{c} / V_{\infty}\right)$. To get the desired blowing ratio, freestream velocity $\left(V_{\infty}\right)$ is kept constant at $15.28(\mathrm{~m} / \mathrm{sec})$ at the freestream inlet, whilst the coolant inlet velocity $\left(\mathrm{V}_{\mathrm{c}}\right)$ is changed at the plenum inlet. The coolant velocity at the plenum inlet for the upstream sister holes for various blowing ratios is given below in Table 5.2. It is to note that the upstream sister holes in the current investigation required 2.125 more coolants than that of a single cylindrical hole since the sister holes have a diameter $=0.75 \mathrm{D}[10]$.

Table 5.2: The coolant velocity at the plenum inlet for the upstream sister holes.

\begin{tabular}{|c|c|}
\hline Blowing Ratio M & Coolant Velocity $(\mathbf{m} / \mathbf{s e c})$ \\
0.3 & 0.015 \\
0.5 & 0.025 \\
1 & 0.050 \\
1.5 & 0.076 \\
2.0 & 0.101 \\
2.5 & 0.126 \\
\hline
\end{tabular}

The computational analysis is carried out on FLUENT-ANSYS 17.1, which is a finite volume solver that solves the 3D Reynolds Averaged Navier-Stokes equations and the energy equation. First, the Realizable k-epsilon is used to model the turbulence of the flow field in combination with enhanced wall treatment (EWT). Because of the significant impact of the mesh quality in the accuracy of the numerical results, the mesh is constructed very carefully in ANSYS-ICEM 17.1 using the unstructured hexahedral mesh feature, and O-grids are created around the cooling holes. To resolve the whole viscous sublayer, enhanced wall treatment is employed, which required that ( $y+$ less than one). Therefore, the density of the generated mesh in the near-wall has been carefully established to satisfy the $(\mathrm{y}+)$ requirement. A sample of the typical mesh generated is shown in figure $5.2 \mathrm{a}$ ) and b). The applied boundary conditions to the freestream channel, cooling holes, and the plenum is depicted in Figure 5.3, while all of the remaining computational model surfaces are identified as adiabatic walls.

The computational analysis is performed as time-independent, pressure base, and doubleprecision analysis. Consistent with the experiment, the working fluid is set as air, which is considered to be incompressible and ideal gas flow. The discretization scheme for the RANS 


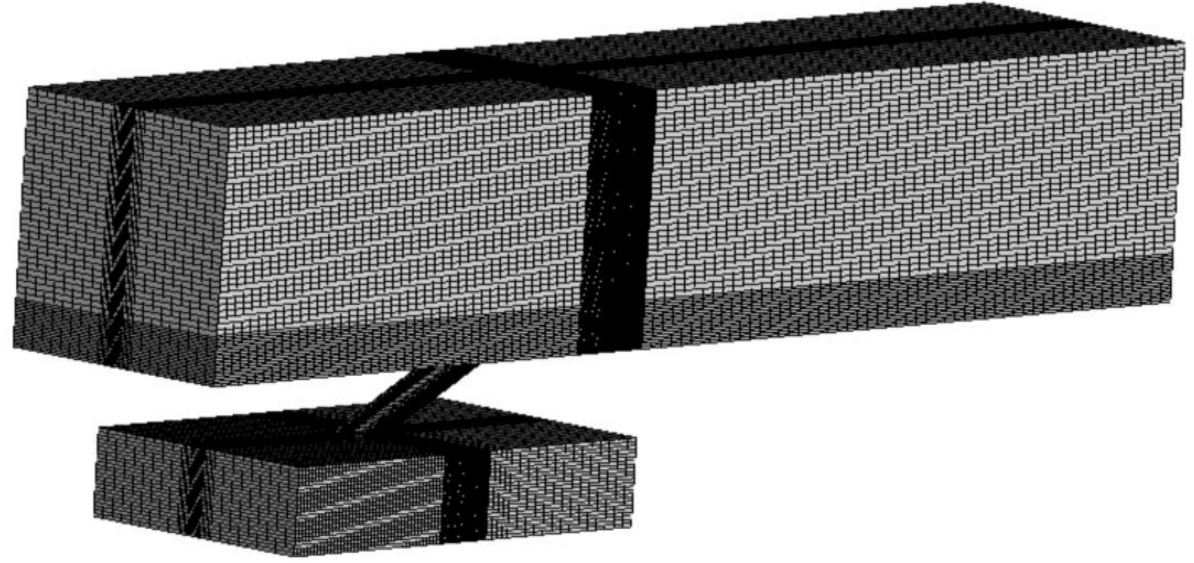

a) Isometric view

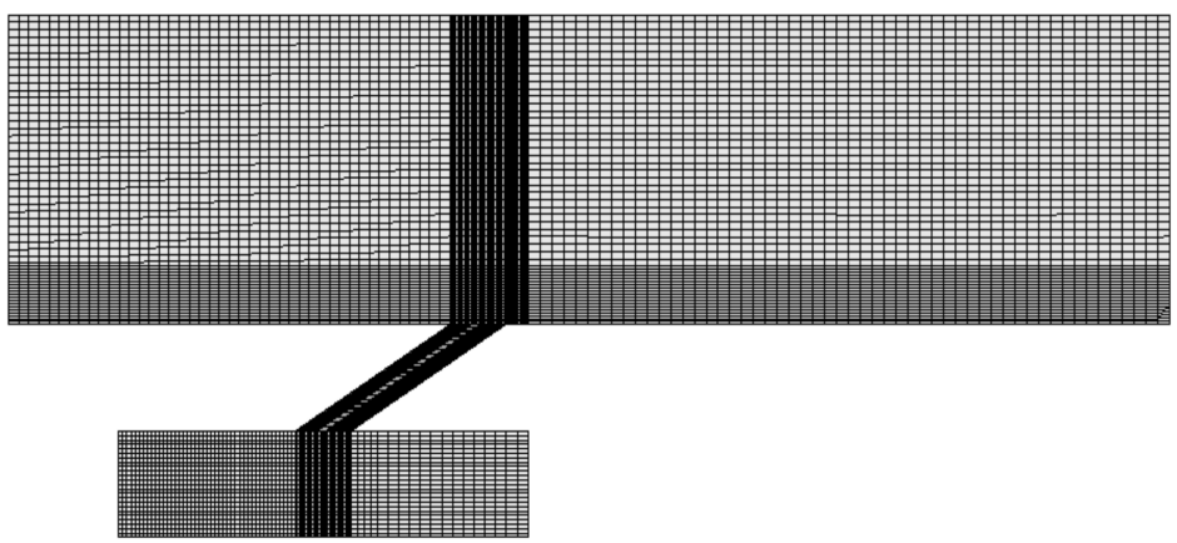

b) Front view.

Figure 5.2: The mesh generated for the upstream sister holes. (Not to scale)

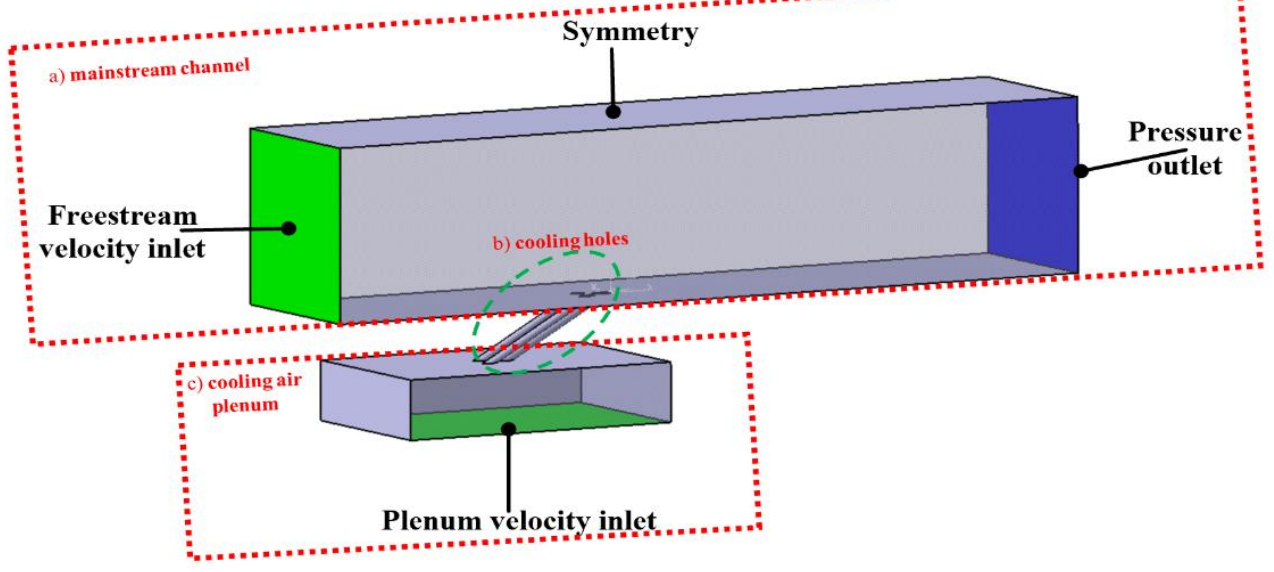

Figure 5.3: The 3D computational model boundary conditions 
equations, energy equation, and turbulence model equations is the second-order upwind scheme. The SIMPLEC algorithm is used as a solution method for the velocity-pressure coupling. Normalized residuals are set as the main the convergence criteria whereas the convergence of the numerical solutions is established when nonnormalized residuals of the continuity equation and all velocity components are less than $\left(1 \times 10^{-4}\right)$ and energy equation residuals are less than $\left(1 \times 10^{-}\right.$

$\left.{ }^{6}\right)$. High order term of relaxation $(0.75)$ is considered to assist the solution convergence stability.

\subsection{Results and Discussion}

In this section, the numerical results of the upstream sister holes will start by first presenting the mesh independency study, which is done by predicting the laterally averaged effectiveness at $\mathrm{M}=0.5$. Second, the prediction of the lateral effectiveness performance from five turbulence models available on ANSYS-FLUENT is evaluated at two blowing ratios $\mathrm{M}=0.5$ and 1.0, whereas the results are compared with the experimental data. Third, the near-wall modeling effect on the numerical results is presented by examining three wall functions along with the RKE turbulence model. Finally, the prediction of the area-averaged film effectiveness, as well as the local film cooling effectiveness at the plate surface is shown at various blowing ratios. A discussion of the possible reasons for the differences and similarity amongst the studied turbulence models are also included. Very initial results of the current study are presented by Abd Alsalam and Jubran in [101].

\subsubsection{Grid Independency Study}

The experimental results of the upstream sister holes by $\mathrm{Wu}$ et al. [10] are primarily geared towards the holes adjacent area unto $(X / D=11)$ along with the lateral spacing of $( \pm 4 D)$ in the y-direction. Moreover, the major parameters that used in the experimental analysis are the laterally averaged film cooling effectiveness and the area-averaged film effectiveness; which are done both numerically and experimentally, at several blowing ratios $\mathrm{M}=0.3-2.5$ [10]. Consequently, The mesh sensitivity study is carried out to predict the laterally averaged effectiveness of the upstream sister holes at a blowing ratio of $\mathrm{M}=0.5$, as depicted in Figure 5.4. As the plot shows, there were five different grid sizes created whereby the grid density is gradually increased from coarse grid to a very fine grid whilst the range of their cells count is within the range of $\left(\approx 6.57 \times 10^{5}\right)$ cells to $\left(\approx 2.36 \times 10^{6}\right)$ cells. Also, the plot shows that prediction of the lateral effectiveness using the very fine grid; consisting of $\left(\approx 2.36 \times 10^{6}\right)$ cells, is nearly 
similar to the result obtained by fine mesh; consisting of $\left(\approx 2 \times 10^{6}\right)$ cells. Therefore, the solution through a fine grid with $\left(\approx 2 \times 10^{6}\right)$ cells is considered the grid that accomplishes the grid independence study and will be used to complete the turbulence models investigation.

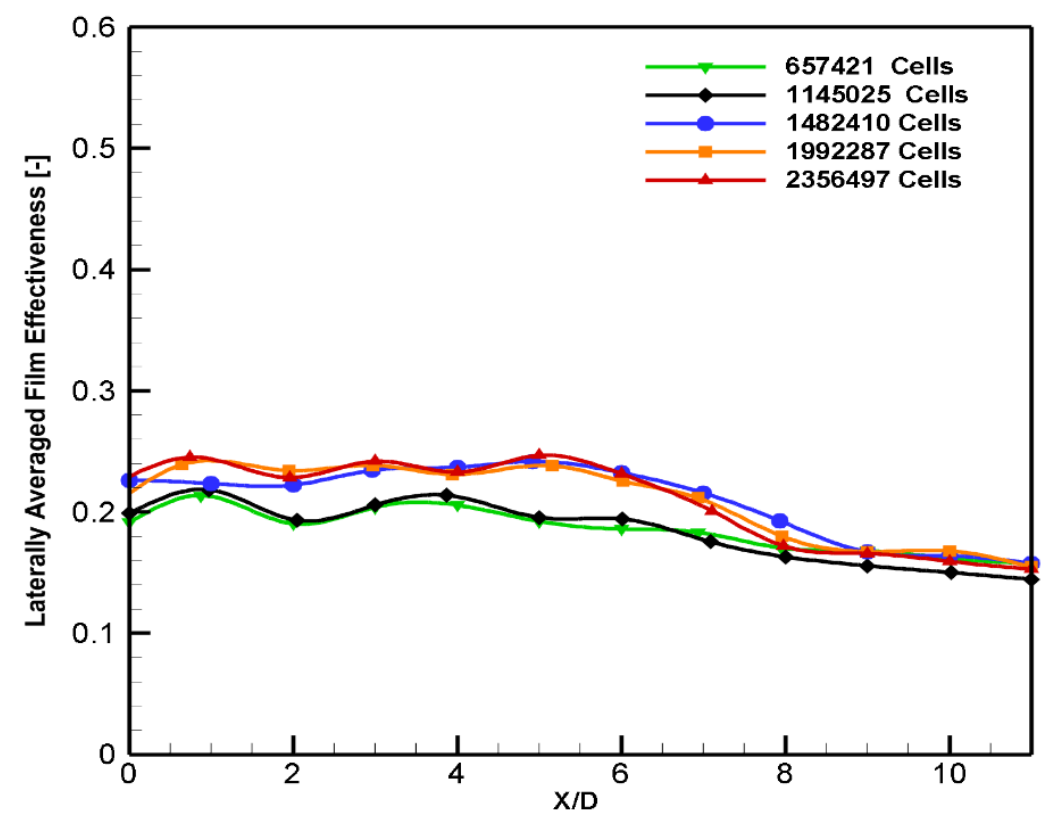

Figure 5.4: Mesh sensitivity analysis at $M=0.5$

\subsubsection{Turbulence Models and Near-Wall Treatments Prediction Results}

The thermal and flow fields of sister holes film cooling are naturally very complicated due to the involvement of the strong secondary flow whereas when it gets mixed with the mainstream, it leads to various degrees of interaction and based on the operating conditions it changes the thermal performance. Therefore, for an accurate and well prediction of the sister holes cooling performance, a proper and careful selection of the turbulence model and near-wall treatment is heavily required.

The prediction of the laterally averaged effectiveness of the upstream sister holes through various turbulence models at low blowing ratio is shown in Figure 5.5. The plot exhibits that all of the used turbulence models have presented kind of diverse behaviour in capturing the laterally averaged effectiveness; precisely in the holes vicinity area at $(\mathrm{X} / \mathrm{D}<2)$. However, further downstream all of the examined turbulence models show a slight underprediction of lateral performance that doesn't go above $(\sim 12 \%)$ as a result of a deficiency in predicting the lateral 
spreading of the coolant jet. By comparing the prediction capability of three categories of k- $\varepsilon$ turbulence models in capturing the lateral performance, RKE outperforms the two other turbulence models; the RNGKE and SKE, and offers an excellent numerical results in the hole vicinity region at $(\mathrm{X} / \mathrm{D} \leq 5)$ that are well agreed with the experimental data as well as it captures the general trend of the lateral effectiveness distribution. Further downstream; however, RKE underestimates the lateral effectiveness like other turbulence models. Alternatively, RNGKE and SKE yield a significant overprediction of the lateral performance whereas immediately at the hole exit the overprediction is almost 1.75 times more than that of the experimental data, and then the overprediction gradually decreases in the holes vicinity region at $(0<\mathrm{X} / \mathrm{D}<2)$ to approach the experimental data. Interestingly, at $(X / D=2)$, the three models of $k-\varepsilon$ present similar results and exceptionally match the experimental data. The SA model; which is a one equation turbulence model, shows numerical results that are fairly comparable to that obtained by RKE, while it slightly overpredicts the performance in the hole vicinity area at $(\mathrm{X} / \mathrm{D}<2)$. On the other hand, the SA model illustrates better predictions, even better than that achieved through RKE further downstream at $(X / D \geq 5)$. Moreover, within the hole adjacent region at $(2<X / D<5)$, SA model offers the poorest prediction of the lateral performance in contrast to the other turbulence models and the experimental data. Fig. 5.5 also illustrates that the Reynolds stress model RSM) is not offering any presumed better prediction of the lateral performance and consistently underestimates the effectiveness of the whole downstream area. Additionally, RSM has shown the worsen underprediction case of the lateral performance in the downstream region at (X/D > 4). Harrison and Bogard [76] have previously found that the RSM had not expressed any improvement in predicting the lateral performance in comparison to the $\mathrm{k}-\varepsilon$ models for the single cylindrical hole. Therefore, in general, at a low blowing ratio; $M=0.5$, it can be stated that the performance attained by RKE model is considered the best performance and the numerical results are well predicted and agreed with lateral effectiveness experimental data of $\mathrm{Wu}$ et al. [10]. 


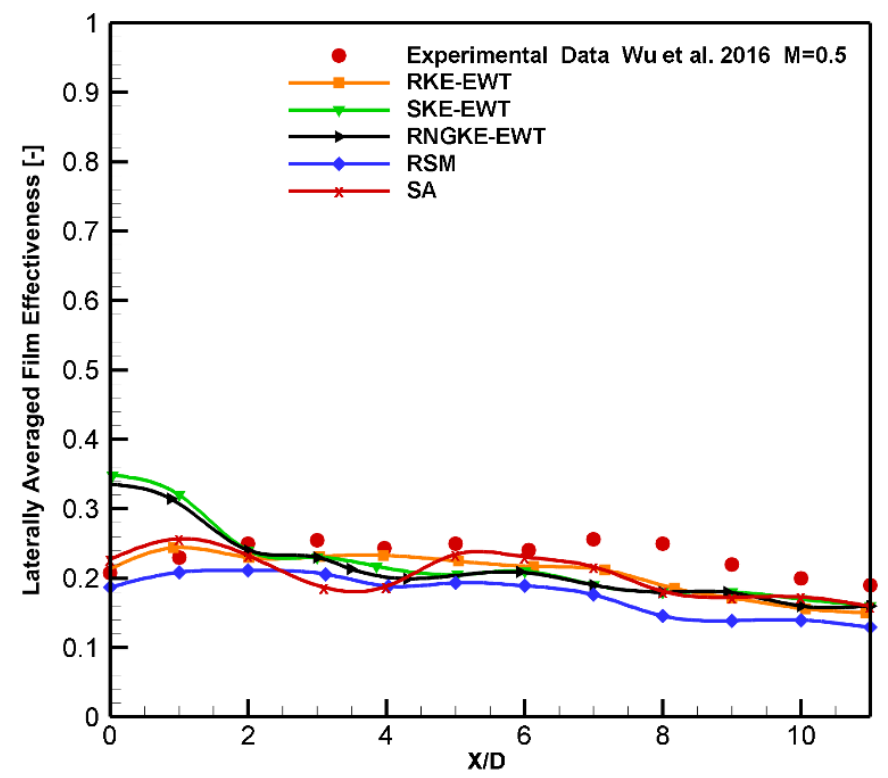

Figure 5.5: The turbulence models prediction of upstream sister holes laterally averaged film cooling effectiveness at $\mathrm{M}=0.5$ and D.R= 1.05.

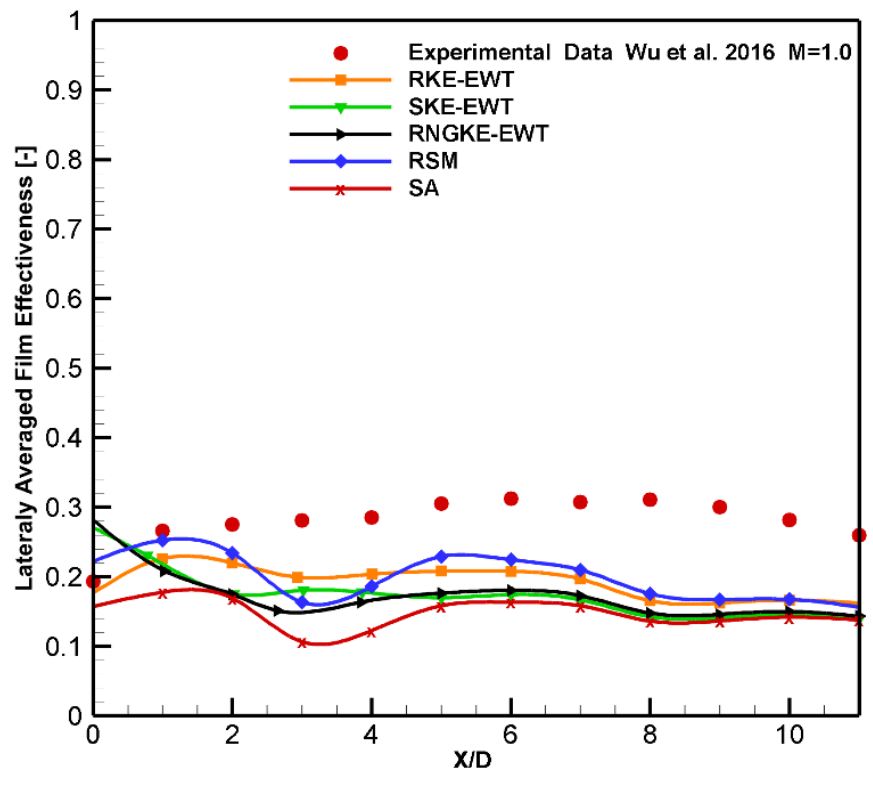

Figure 5.6: The turbulence models prediction of upstream sister holes laterally averaged film cooling effectiveness at $M=1.0$ and $\mathbf{D . R}=\mathbf{1 . 0 5}$.

The numerical prediction of the upstream sister holes laterally averaged effectiveness at $\mathrm{M}=1.0$ is depicted in Figure 5.6. The plot shows that in the hole vicinity area at $(\mathrm{X} / \mathrm{D}<2)$ the examined turbulence models present a notable variation in capturing the lateral spreading of the coolant flow, further downstream; however, the underprediction is the general trend for all investigated models. Moreover, in the hole vicinity area at $(\mathrm{X} / \mathrm{D} \leq 2)$, Fig. 5.6 exhibits that $\mathrm{RKE}$ and RSM models offer somewhat a reasonable prediction of the experimental data, whereas RKE is slightly underpredicted the performance, while RSM shows a minor overprediction. However, the prediction results of RKE are better than the RSM in capturing the experimental data. In the far downstream region, none of the studied turbulence models are likely to capture the general trend of the lateral performance. Hassan and Yavuzkurt [75] reported that k- $\boldsymbol{\varepsilon}$ turbulence models are typically presented an underprediction of lateral effectiveness distribution as a result of the underlying hypothesis of isotropic of the turbulent viscosity [75]. Even though the RSM is used as an anisotropic turbulence model while looking for a better prediction of the results, it underpredicts the lateral performance similar to other investigated models; k-epsilon models and SA model. SA model entirely failed to produce any meaningful results, whereas the worst 
underprediction of the lateral performance for the whole downstream region is presented by this turbulence model and the deviation between the experimental data and SA numerical results is found nearly $45 \%$.

According to the reasonable prediction of the lateral performance attained by RKE model at low blowing ratio $M=0.5$, it is selected to perform the analysis on how the near-wall treatment affects the prediction of sister holes lateral effectiveness in comparison to the experimental data of [10] at $\mathrm{M}=0.5$. The near-wall treatments are modelled using three wall functions available on FLUENT-ANSYS along with the RKE, namely: standard wall function (SWF), scalable wall function (ScWF), and enhanced wall treatment (EWT), as shown in Figure 5.7. Note that, the generated mesh of the upstream sister holes computational model has been updated to fulfill the $y+$ requirements of the examined near-wall functions. As stated previously, $(y+<1)$ is required for enhanced wall treatment, while $(30<y+<300)$ is the limit for the standard wall function and scalable wall function [67] [78].

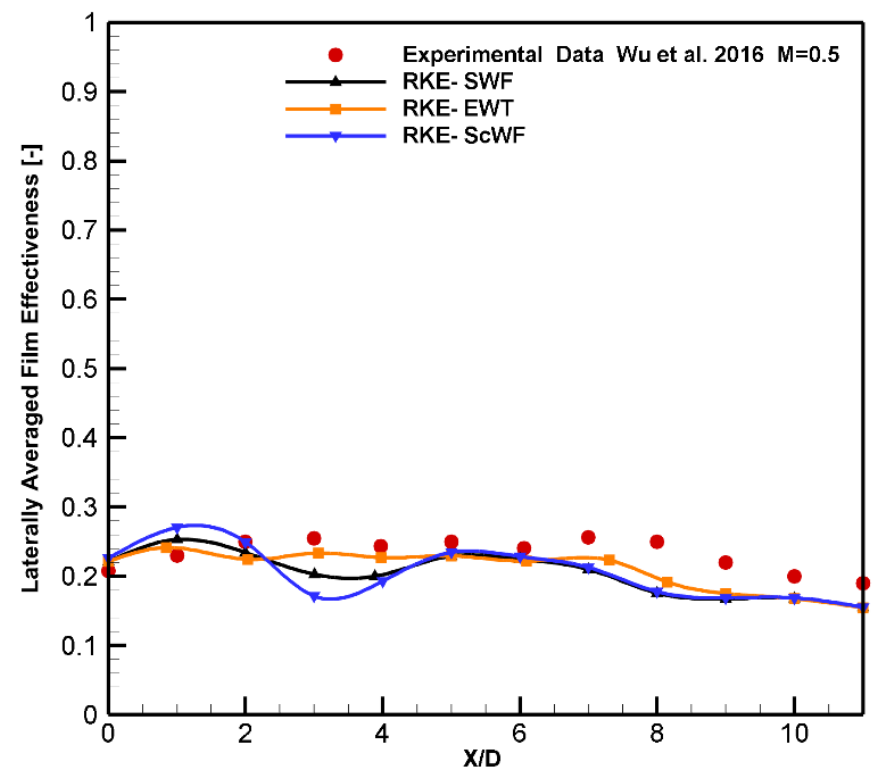

Figure 5.7: The near-wall treatments prediction of the lateral effectiveness of the upstream sister holes at $\mathrm{M}=0.5$.

It is generally believed that the centerline and lateral performance of discrete cylindrical hole at low and high blowing ratio are reasonably well captured and predicted through the standard wall function along with relizable k- $\varepsilon$ [78] [94]. But, in the present investigation, the prediction of SWF and ScWF of lateral performance of upstream sister holes, depicted in Fig. 5.7 , failed to produce any meaningful result in the hole vicinity region at $(X / D \approx 5)$. Whereas 
they both present some sort of oscillation pattern; in which an overprediction of the lateral performance is shown at $(X / D<2)$, and then after an underprediction is present in most of the downstream area. Interestingly, at $(\mathrm{X} / \mathrm{D} \geq 5)$ the $\mathrm{SWF}$ and the ScWF offer the same performance while they underestimated the lateral spreading of the coolant. On the other hand, EWT is clearly offering the best numerical results that considerably well predict the lateral performance up to the $(\mathrm{X} / \mathrm{D} \leq 6)$, whilst further downstream, it is also slightly underpredicted the performance but not as much as the two other wall functions. Moreover, it is interestingly to notice that the three nearwall functions; (SWF), (ScWF), and (EWT) exhibit an excellent and alike performance within the region of $(5 \leq X / D \leq 6)$ in comparison to experimental data. Therefore, it might be argued that for a better prediction of sister holes cooling performance, the best approach is to resolve the whole wall boundary layer to the viscous sublayer through the enhanced wall treatment along with the Realizable k-epsilon turbulence model. Harrison and Bogard [102] studied different hole configurations; narrow and wide trenched holes, and their findings are consistent with the current findings whereas they were able to obtain a good prediction to the experimental data and captured the separation of the coolant jet using the RKE with enhanced wall treatment.

Wu et al. [10] found that there is a substantial reduction in the lateral cooling performance in the holes vicinity region of the upstream sister holes at high blowing ratio $\mathrm{M}=2.0$ as a result of the jet lift-off. Accordingly, it is ultimately important to check the ability of Relizable k- $\varepsilon$ turbulence model with the enhanced wall treatments in capturing the jet lift-off phenomena. Figure 5.8 a)-d) demonstrates the prediction of the lateral effectiveness at several blowing ratios $\mathrm{M}=0.3,1.5,2.0$, and 2.5 in contrast to the experimental data. Fig 5.8 a) exhibits that at a low blowing ratio; $M=0.3$, the numerical results quite well capture the lateral performance in contrast to the experimental data, but at $(\mathrm{X} / \mathrm{D}>6)$ a slight underprediction is observed. The prediction of numerical results at $\mathrm{M}=0.3$ demonstrates a comparable trend to the performance predicted herein at $\mathrm{M}=0.5$, which is shown in Fig. 5.5. 


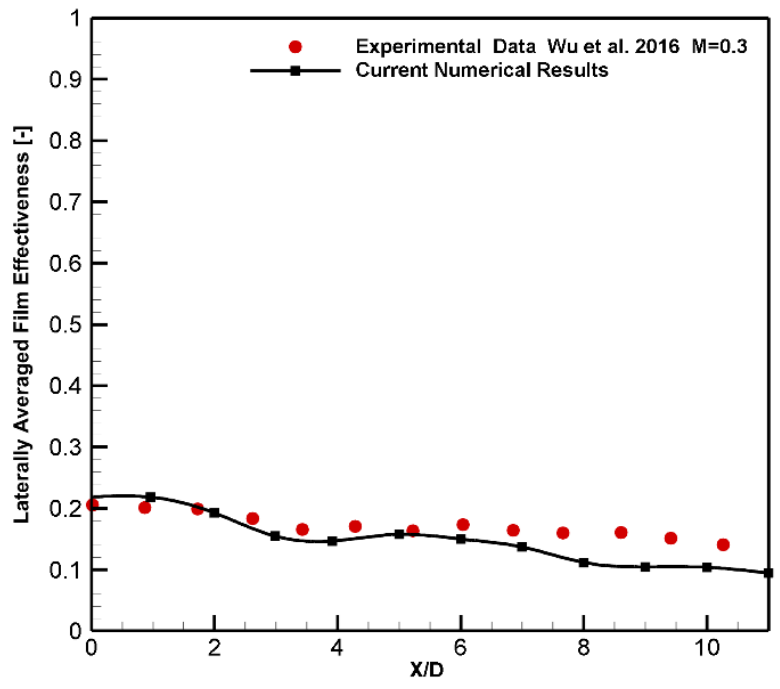

a) $\mathrm{M}=0.3$

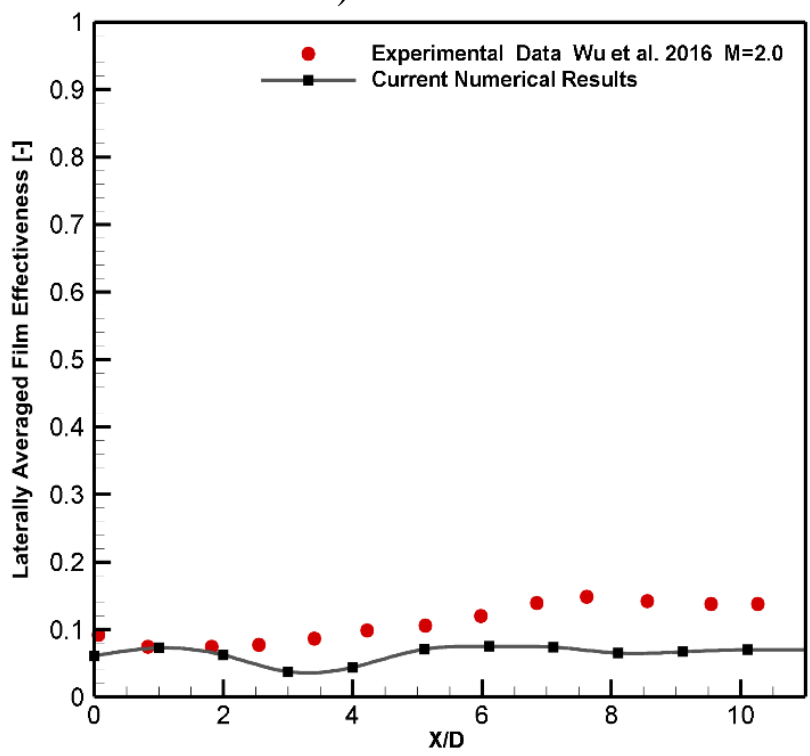

c) $\mathrm{M}=2.0$

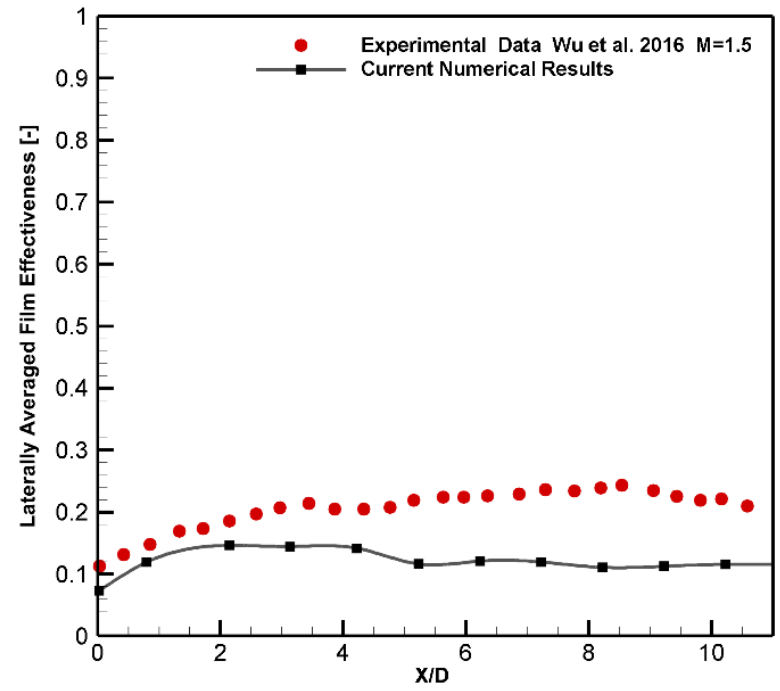

b) $\mathrm{M}=1.5$

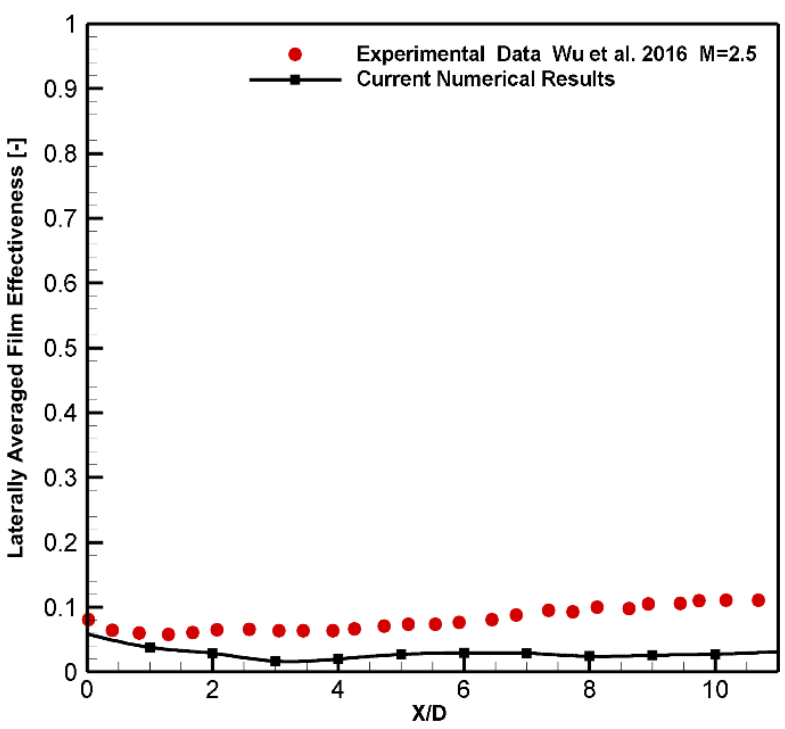

d) $\mathrm{M}=2.5$

Figure 5.8: a) - d) The prediction of the upstream sister holes laterally averaged film cooling effectiveness in contrast to the experimental data of Wu et al. [10] at various blowing ratios.

At high blowing ratios; $M=1.5,2.0$, and 2.5, the predicted results of lateral effectiveness manifest a systematic underprediction for the whole downstream area, as revealed in Figure 5.8 b), c), and d). Surprisingly, at $M=2.0$, in the vicinity holes region at ( $1 \leq X / D \leq 2)$ numerical results and the experimental data are nearly similar, while further downstream at (X/D > 4 ), the numerical results show almost a plateau trend which might be a result of the intensive mixing that arose between the coolant jet emerged from the sister holes and the freestream. The lowest 
performance is shown at $\mathrm{M}=2.5$, as depicted in Fig. $5.7 \mathrm{~d}$ ), which is attributed to the strong mixing and deep penetration of the coolant jet that has a very high jet momentum into the freestream. As a result, a dramatic deterioration in the coolant coverage and effectiveness is attained.

Nevertheless, the CFD results presented here have revealed an underprediction of the later effectiveness of upstream sister holes at high blowing ratios $M=1.5-2.5$, while good numerical results are attained at low blowing ratios. It can be fairly argued that a reasonable prediction of the lateral cooling performance is accomplished. Overall, the underprediction of the lateral cooling performance of the upstream sister holes at high blowing ratios might be caused by two main reasons. The first reason could be the isotopic assumption in the applied turbulence model; Relizable $\mathrm{k}-\varepsilon$, whereas it is typically documented in many of the previous numerical studies. Recently, Stratton and Shih [103] found that RANS turbulence models have the tendency to underpredict the near-wall temperature gradient, which caused a significant deficiency in predicting the lateral spreading of coolant jet. The other reason that possibly caused the discrepancy between the numerical results and the experimental data is the conduction error [94], whereas the test surface of the flat plate was made from polymethylmethacrylate (PMMA) with thermal conductivity of $0.19 \mathrm{~W} / \mathrm{mK}$. This conduction error was ignored in the experimental work of Wu et al. [10]. It was reported by Han [4] that the detailed experimental measurement of film cooling effectiveness distributions using the liquid crystal technique is suffering from heatconduction correction problems, which can be avoided by utilizing advanced technologies such as pressure-sensitive paint method.

A comparison of the area-averaged film cooling effectiveness predicted results of the upstream sister holes to that of $\mathrm{Wu}$ et al. [10] in which the results are available experimentally and numerically are presented in Figure 5.9. The plot obviously manifests that the current numerical results are practically matching to the numerical results of $\mathrm{Wu}$ et al. [10], although the standard wall function with RKE is used in their numerical analysis. Moreover, the two numerical results demonstrate a somewhat of underprediction at high blowing ratios and comparable trend in contrast to the experimental data. Yet, it can be concluded that Relizable k- $\varepsilon$ turbulence model presents its ability in predicting the lateral effectiveness distribution and the area-averaged film effectiveness of the upstream sister holes at low blowing ratio, but at high blowing ratio, it is not capable of capturing the cooling performance to some degree; mainly in the far downstream region. 


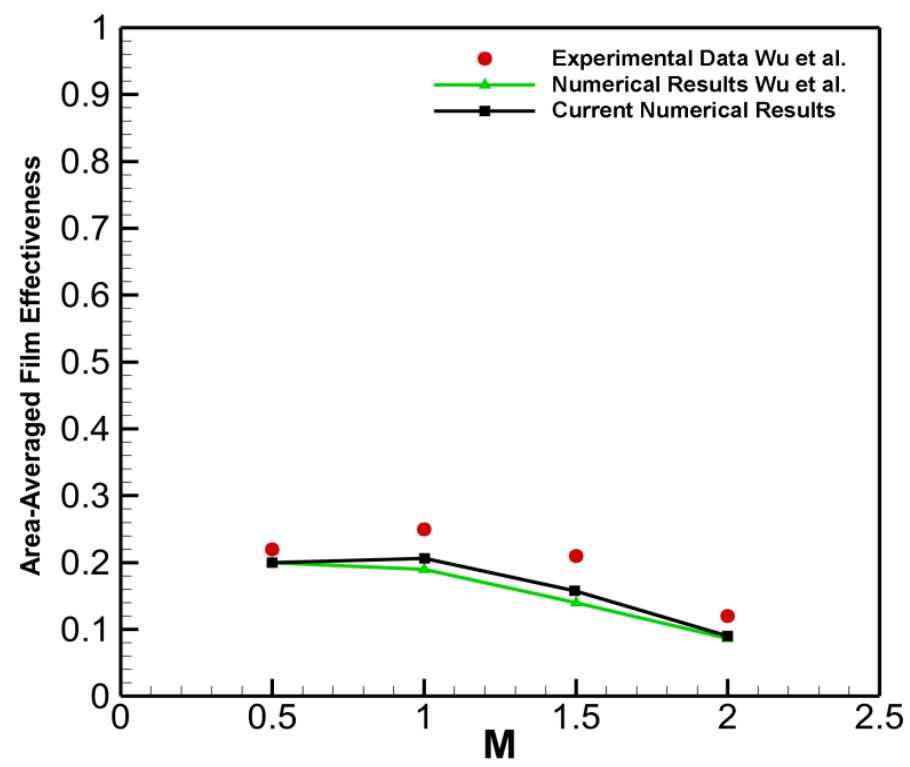

Figure 5.9: The comparisons of the area-averaged film effectiveness to that of $\mathrm{Wu}$ et al. [10].
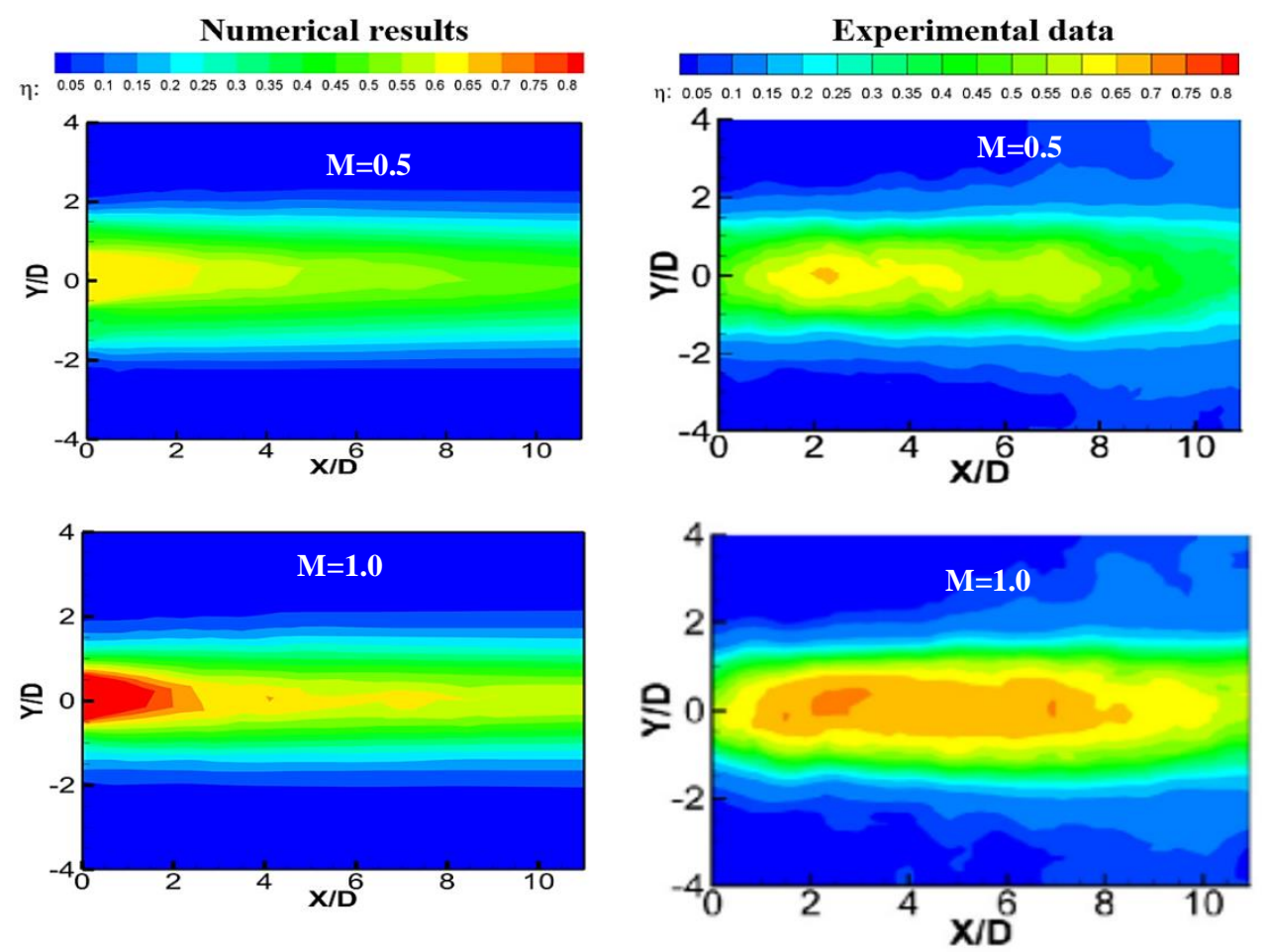

Figure 5.10: The comparisons of the film cooling effectiveness distribution at $M=0.5$ and $M=1.0$ numerical results (left) and experimental data of $W u$ et al. [10](right).

The upstream sister holes have shown the best area-averaged film effectiveness performance at two blowing ratios $\mathrm{M}=0.5$ and 1.0, as depicted in Fig. 5.9, while Wu et al. [10] 
stated that the optimal effectiveness and coolant coverage is attained at blowing ratio $\mathrm{M}=1.0$. As such, the prediction of local film cooling effectiveness is performed at $\mathrm{M}=0.5$ and $\mathrm{M}=1.0$, as depicted in Figure 5.10. Fig. 5.10 exhibits the contours results that predicted the local film effectiveness distribution (left column) of the upstream sister holes at the plate surface in comparison to the experimental data (right column) of Wu et al. [10]. The effectiveness contours vertically cover the region of $\mathrm{Y} / \mathrm{D}= \pm 4$, while it horizontally covers the entire holes downstream area $(X / D=0$ to 11$)$. Fig. 5.10 shows that at $M=0.5$ the numerical results predict the lateral coolant distribution pretty well whereas the coolant covers the vertical domain of $(\mathrm{Y} / \mathrm{D}= \pm 2)$ and those results are in a good agreement with the experimental data. Moreover, close to the center of the vertical domain, the numerical results capture the coolant attachment region at the plate surface, in which it mostly covers the holes downstream area up to $(\mathrm{X} / \mathrm{D}=8)$. On the other hand, the experimental results show a broader lateral coolant distribution at $(\mathrm{X} / \mathrm{D}>6)$, which is underestimated with the current numerical results.

At blowing ratio of $\mathrm{M}=1.0$ Fig. 5.10 presents that the numerical results reasonably predict the lateral coolant distribution in the holes vicinity area at $(X / D<4)$, while it exhibits underprediction of the lateral coolant distribution and the coolant jet attachment region farther downstream. Additionally, the numerical results predicted higher film cooling effectiveness and more coolant concentration in the middle of the vertical domain while it horizontally includes the hole vicinity region at $(X / D \approx 0$ to 2$)$; this does not appear in the experimental results. The experimental data, on the other hand, exhibit a higher coolant coverage that includes the vertical domain of $(Y / D= \pm 0.5)$ within the horizontal region of $(0.5<\mathrm{X} / \mathrm{D}<8)$, which is underpredicted numerically. Thus, it can be interpreted that as the underprediction of the lateral effectiveness is compensated by an overprediction of centerline performance. As such, it would have been ideal to have the experimental data of the centerline effectiveness distribution of the upstream sister holes in the experimental work of Wu et al. [10], which can be used to examine and justify the numerical results that offered sort of divergence close to the centerline of the cooling holes. Overall, the contours of local effectiveness results presented in Figure 5.10 support the previous results of the lateral effectiveness presented herein in Figure 5. 5 and Figure 5.6 whereas at blowing ratio; $M=0.5$, a good agreement is attained between the computational results and experimental data, but at high blowing ratio; $M=1.0$, the computational results underpredicted the lateral effectiveness. 


\subsection{Conclusion}

In this chapter, a numerical investigation is performed in ANSYS- Fluent for the upstream sister cooling holes to examine computational results ability in capturing and predicting the sister holes cooling performance in comparison with experimental data. Five turbulence models available in Fluent are examined at two blowing ratios $\mathrm{M}=0.5$ and $\mathrm{M}=1.0$ to predict the lateral effectiveness, which are realizable k- $\varepsilon$, standard k- $\varepsilon$, RNG k- $\varepsilon$, Reynolds stress model (RSM), and Spalart-Allmaras. Moreover, the realizable $\mathrm{k}-\varepsilon$ is utilized to evaluate the effect of near-wall modeling on the predicted lateral cooling effectiveness at $M=0.5$. It is found that at low blowing ratio, the Realizable k- $\varepsilon$ along with the enhanced wall treatment provided the best numerical results of the lateral effectiveness of the upstream sister holes and notably predicted the experimental data of $\mathrm{Wu}$ et al. [10]. On the other hand, it consistently presents an underprediction to the lateral effectiveness at a high blowing ratio. Reynolds stress model has not given any meaningful numerical results, and it underpredicts the cooling performance at low and high blowing ratios as well as it doesn't show any predication improvements in comparison to the other examined turbulence models. Near-wall modeling notably affects performance prediction whereas resolving the viscous sublayer through the enhanced wall treatment tends to yield more accurate numerical results and well prediction for the upstream sister holes lateral effectiveness performance, in contrast to a standard wall function and scalable wall function. On the other hand, it is computationally more expensive in terms of mesh density and computational time. Overall, for a reasonable prediction of the sister holes film cooling performance, special attention has to be paid to the numerical setup, which includes a proper computational model of flow physics, exact geometry and high-quality grid generation, discretization scheme, and turbulence modeling and near-wall treatments, which has been recommended by Walters and Leylek [41] for a successful film cooling performance prediction. 


\section{CHAPTER SIX: COMPOUND ANGLE UPSTREAM SISTER HOLES FILM COOLING PERFORMANCE}

The motivation behind this chapter is to find a simple and effective cooling hole configuration that improves cooling performance while, at the same time, taking the manufacture time and cost into consideration. It is well known that the cylindrical hole is the most convenient shape of the cooling hole that is widely used in real turbine engine blades; however, it suffers from a low performance at high blowing ratio. Sister holes and compound angle injection of the round hole have been used independently in previous studies, and both demonstrated an improvement in cooling performance over the simple discrete round hole. Also, those two techniques have the advantage of easy manufacturing since they consist of only round holes. Therefore, in this chapter, a novel and simple strategy; compound angle upstream sister holes (CAUSH ), is introduced to increase film cooling performance. (CAUSH) incorporate two techniques that are sister holes (two small round holes placed upstream the primary hole) and compound angle hole, whereas the upstream sister holes are injected at several compound angles. The evaluation of the compound angle upstream sister holes film cooling performance and the flow field will be carried out at different compound angles and blowing ratios.

\subsection{Problem Statement}

It is noteworthy to point out that the reader who goes through each individual chapter in this thesis may realize that number of the geometrical parameters and/or boundary conditions in each chapter may be similar to the previous chapter(s). This is done in purpose to avoid any confusion and to keep the unity of each chapter in the thesis. Also, it should be pointed out herein that the novel compound angle upstream sister holes (CAUSH) are a new film cooling concept, according to the best of the author's knowledge, there are neither experimental data nor numerical results available in literature yet. Therefore, the computational model studied in this chapter is basically built based on a single round hole case in the benchmark experimental work of Sinha et al. [89], which is also similar to the 3D computational model of the discrete round hole case in the study of Khajehhasani and Jubran [68][78]. However, the only different herein to the model in the work of [68][78] is the height of the mainstream channel instead of expanding the model (20D) in ydirection above the plate, (10D) is used. The main cylindrical hole with a diameter of (D)=12.7 $\mathrm{mm}$; highlighted in blue in Figure $6.1 \mathrm{a})$, is injected to the streamwise at $(\alpha)=35^{\circ}$ and has a length 
to diameter ratio $(\mathrm{L} / \mathrm{D})=1.75$. The origin point of the coordinate systems is placed at the trailing edge of the hole (T.E). The computational domain is extended in X-direction 30D downstream the hole (T.E), and 19D upstream the hole leading edge (L.E). Also, the model is extended 3D in the lateral direction to maintain the hole pitch to diameter ratio $(\mathrm{P} / \mathrm{D})=3$. A plenum with dimensions of $(8 \mathrm{D}, 4 \mathrm{D}$, and 3D) in (X, Y, and $\mathrm{Z})$; respectively, is added to the 3D model. To model the compound angle upstream sister holes (CAUSH), two small sister holes with a diameter of $(0.5 \mathrm{D})$ are added upstream the main hole. Those two sister holes are injected symmetrically at several compound angles to the mainstream direction; $\beta=0^{\circ}, 45^{\circ}, 75^{\circ}$, and $90^{\circ}$; while the main hole is only streamwise injected. The sister holes position in $\mathrm{X}$ and $\mathrm{Z}$ directions relative to the center of the main hole is considered a geometrical constraint for each case. Table 6.1 presents the configuration and dimensions of the examined upstream compound angle sister holes cases. Note that, compound angle sister holes injected at $\beta=0^{\circ} ;\left(0^{\circ} \mathrm{CAUSH}\right)$, means that the upstream sister holes are only injected to the streamwise at $\alpha=35^{\circ}$, as shown in Figure 6.1 a) the top view and Figure $6.1 \mathrm{~b}$ ) the side view. Figure 6.2 represents a top view for each CAUSH while the focus is only in the holes vicinity region for a) $45^{\circ} \mathrm{CAUSH}$, b) $75^{\circ} \mathrm{CAUSH}$, and c) $90^{\circ} \mathrm{CAUSH}$, respectively.

Table 6.1: The dimensions of each CAUSH configuration.

\begin{tabular}{|l|cccc||}
\hline Test Case & X/D & Z/D & $(\alpha)$ & $(\beta)$ \\
\hline$\left(\mathbf{0}^{\circ}\right.$ CAUSH) & -0.693 & \pm 0.693 & $35^{\circ}$ & $0^{\circ}$ \\
$\left(45^{\circ}\right.$ CAUSH) & -0.693 & \pm 0.693 & $35^{\circ}$ & $45^{\circ}$ \\
$\left(\mathbf{7 5}^{\circ}\right.$ CAUSH) & -0.693 & \pm 0.772 & $35^{\circ}$ & $75^{\circ}$ \\
$\left(\mathbf{9 0}^{\circ}\right.$ CAUSH) & -0.693 & \pm 0.826 & $35^{\circ}$ & $90^{\circ}$ \\
\hline \hline
\end{tabular}




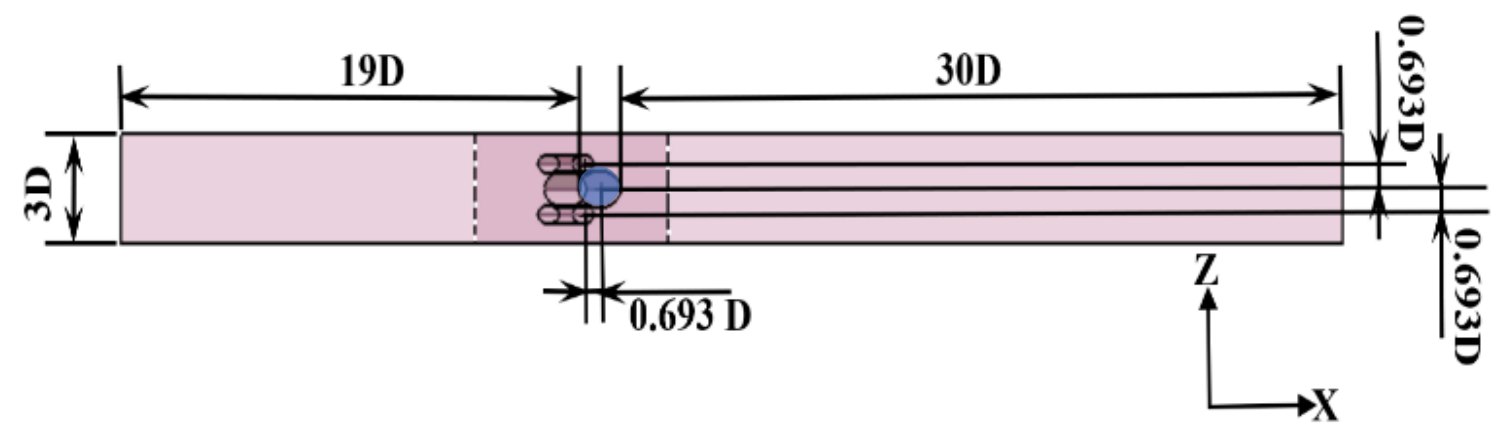

(a) Top view

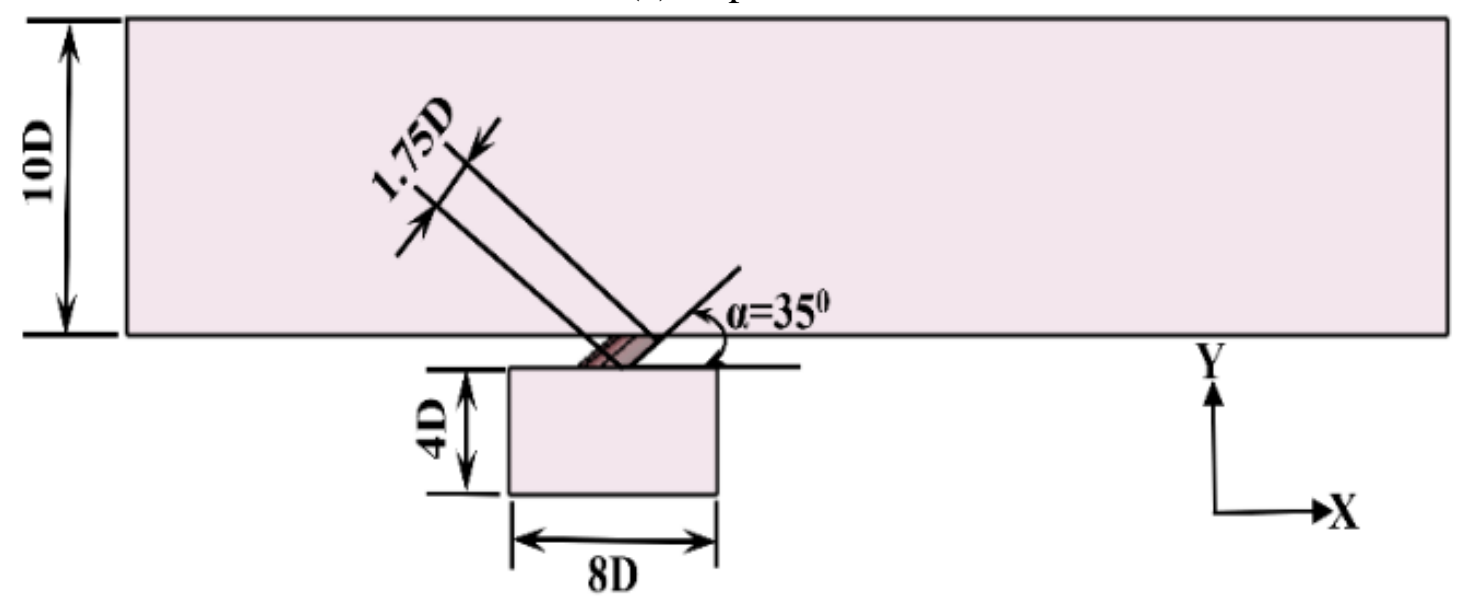

(b)Side View

Figure 6.1: The computational model for $0^{\circ}$ (CAUSH) (a) top view, (b) side view. (Not to scale).

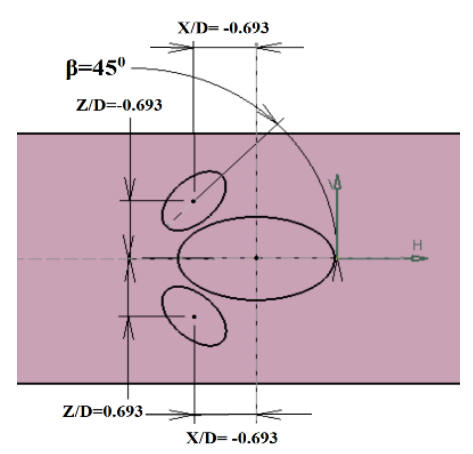

a) $45^{\circ} \mathrm{CAUSH}$

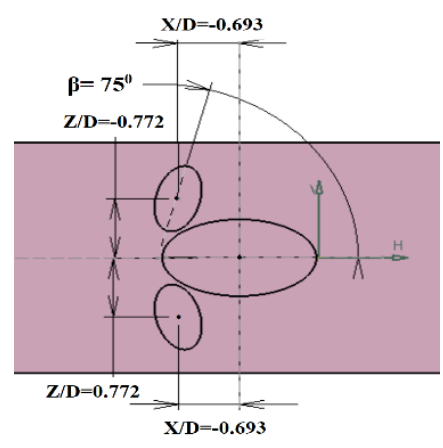

(d) $75^{\circ} \mathrm{CAUSH}$

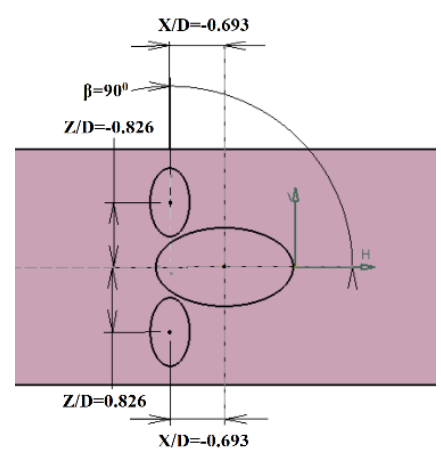

c) $90^{\circ} \mathrm{CAUSH}$

Figure 6.2: A top view focusing on the cooling holes vicinity region for

a) $45^{\circ} \mathrm{CAUSH}$, b) $75^{\circ} \mathrm{CAUSH}$, and c) $90^{\circ} \mathrm{CAUSH}$, respectively. (Not to scale). 


\subsection{Simulation parameters and Computational Overview}

The boundary conditions applied to the computational model in the present analysis are selected to match the boundary conditions in the numerical study of Khajehhasani and Jubran for the discrete round hole case [68][78], which were also built based on the experimental measurements data of Sinha et al.[89]. The freestream temperature $=300 \mathrm{~K}$ and coolant temperature $=150 \mathrm{~K}$ are applied at the freestream and coolant inlet, respectively, while they satisfy a density ratio of two $(D . R=2)$. Since the turbulence boundary conditions significantly affect the accuracy of numerical results, the turbulence intensity and the viscosity ratio $\left(\mu_{t} / \mu\right)$ are utilized at the freestream and the coolant inlets [78], and their values are listed in Table 6.2. Four blowing ratios are tested for each $\mathrm{CAUSH}$, in which $\mathrm{M}=0.25$ and 0.5 are the low blowing ratios cases, and $\mathrm{M}=1.0$ and 1.5 are the high blowing ratios cases. Note that, compound angle upstream sister holes required one and half more coolant than that of the single round hole. As such, the desired value of the blow ratio for the compound angle sister holes can be attained by maintaining a constant freestream velocity $=20(\mathrm{~m} / \mathrm{sec})$ at the freestream inlet whilst changing the coolant velocity at plenum inlet. The coolant inlet velocity at the plenum inlet for the discrete hole and compound angle sister holes is shown in Table 6.3. The boundary conditions applied to the model are defined in Figure 6.3.

Table 6.2: The mainstream and coolant flow parameters

\begin{tabular}{|l|c|}
\hline \multicolumn{1}{|c|}{ Property } & Value/units \\
\hline Free stream velocity & $20(\mathrm{~m} / \mathrm{sec})$. \\
Freestream temperature & $300 \mathrm{~K}$ \\
Freestream turbulence intensity & $0.2 \%$ \\
Coolant temperature & $50 \%$ \\
Coolant turbulence intensity & $150 \mathrm{~K}$ \\
Coolant viscosity ratio & $2.0 \%$ \\
Density ratio (D.R) & $30 \%$ \\
Operating pressure & 2 \\
\hline
\end{tabular}


Table 6.3: The coolant velocity at the plenum inlet.

\begin{tabular}{|c|c|c|}
\hline Blowing Ratio M & $\begin{array}{c}\text { Single hole } \\
\text { Vc }(\mathbf{m} / \mathbf{s e c})\end{array}$ & $\begin{array}{c}\text { Compound sister holes } \\
\text { Vc }(\mathbf{m} / \mathbf{s e c})\end{array}$ \\
\hline $\mathbf{0 . 2 5}$ & 0.082 & 0.123 \\
$\mathbf{0 . 5}$ & 0.164 & 0.245 \\
$\mathbf{1}$ & 0.327 & 0.491 \\
$\mathbf{1 . 5}$ & 0.491 & 0.736 \\
\hline
\end{tabular}

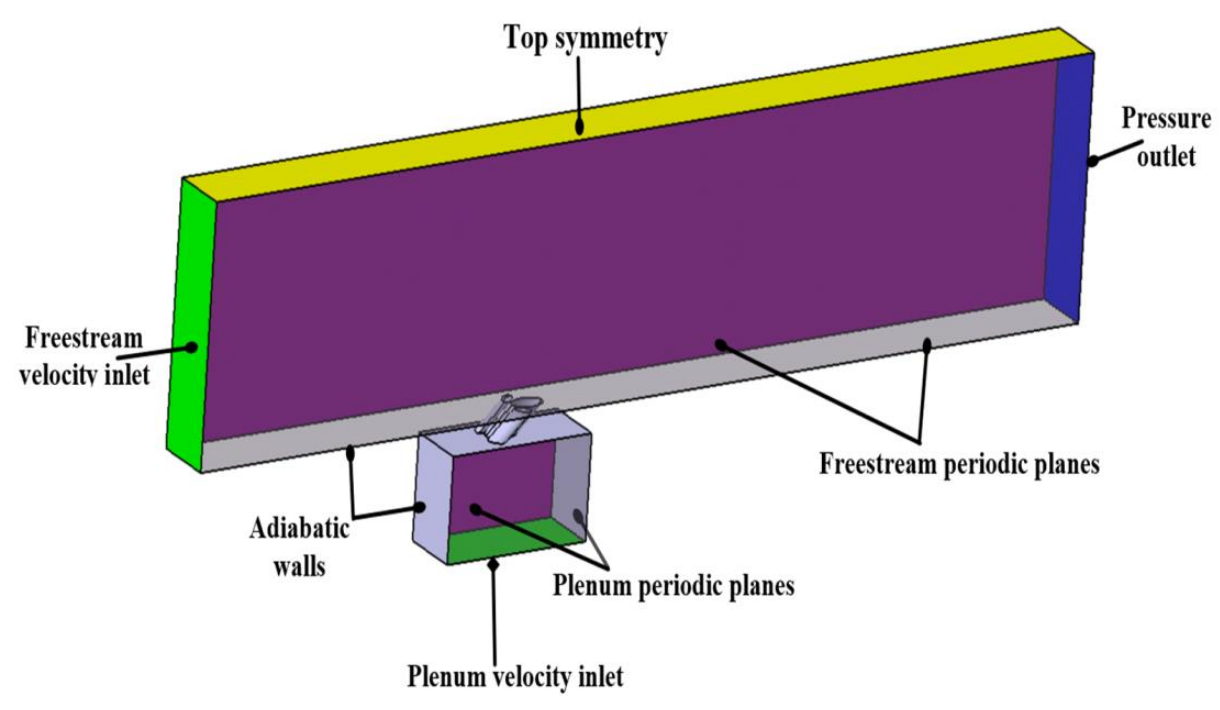

Figure 6.3: The 3D computational domain boundary conditions showing the $45^{\circ}$ CAUSH case .

The governing equations described the thermal and flow field of the compound angle upstream sister holes are the 3D Reynolds Averaged Navier-Stokes equations and the energy equation presented in chapter three. These equations are solved numerically using FLUENTANSYS 17.1 software package. The turbulence of the flow field is modelled through the realizable k-epsilon turbulence model, while the near-wall modeling is done using the enhanced wall treatment. The computational model grids are created on ANSYS-ICEM 17.1, whereas the unstructured hexahedral mesh technique is applied. For a good resolution and high mesh quality in the area near the cooling holes, the O-grids are wrapped around the cooling holes. Special 
attention has also been paid to the mesh generated near the wall for a better resolution of the boundary layer. The mesh is created very fine near the wall and the adjacent area of cooling holes region to achieve the $(\mathrm{y}+)$ required for the enhance wall treatment, which is $(y+<1)$. On the other hand, a coarser mesh is progressively created far from the wall to reduce the computational cost. Figure 6.4 represents a sample of the generated mesh for the $0^{\circ}$ (CAUSH) case.

The computational cases are run in ANSYS-FLUENT 17.1; which is a finite volume solver, and the analysis is processed as time-independent, pressure base, and double-precision analysis. Consistent with the experiment, the working fluid is set as air and considered to be incompressible, ideal, and Newtonian flow. The discretization scheme of the momentum, energy, and turbulence model equations is the second-order upwind scheme. The SIMPLEC algorithm is used as a solution method for the velocity-pressure coupling. The convergence of the numerical solution is considered to be established when: (1) normalized residuals for the continuity equation and all velocity components are less than $\left(1 \times 10^{-4}\right)$ and energy equation normalized residuals are less than $\left(1 \times 10^{-6}\right),(2)$ the mass and energy imbalances for the entire computational domain is less than $0.001 \%$.

\subsection{Results and Discussion}

The results section will start by showing the mesh sensitivity study, and examine the

reliability of the current numerical results, then after the thermal and flow field assessments of the compound angle upstream sister holes film cooling performance is presented.

\subsubsection{Sensitivity Study and Numerical Results Validation}

The parameter used to examine the grid sensitivity is the centerline effectiveness at low blowing ratio $\mathrm{M}=0.5$ for the single round hole case, while the results also validated against the centerline effectiveness data in the experiment of Sinha et al. [89], as shown in Figure 6.5. As the plot shows, the centerline effectiveness attained from fine mesh that consists of $\left(1.45 \times 10^{6}\right)$ cells is nearly identical to that obtained using the very fine mesh that consists of $\left(2.505 \times 10^{6}\right)$ cell and the results of both mesh sizes very well agree with the experimental data. The coarse mesh and the medium mesh, however, show somewhat of discrepancy in comparison to the experimental data. Therefore, the sensitivity study is established through fine-mesh with 
$\left(1.45 \times 10^{6}\right)$ cells. Note that, in chapter four, the same grid sizes are used for the mesh sensitivity evaluation; however, it was performed at high blowing ratio $\mathrm{M}=1.0$.

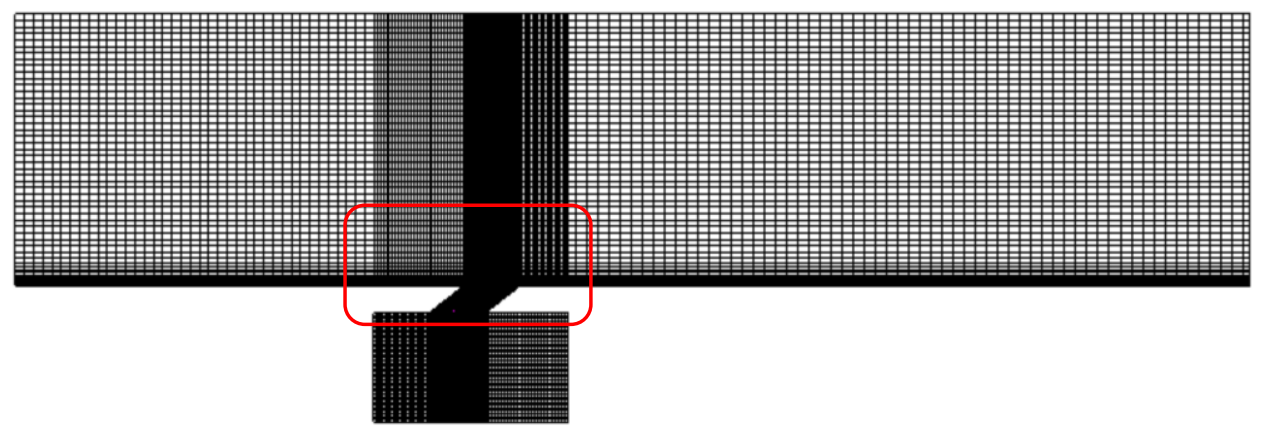

a) Front view.

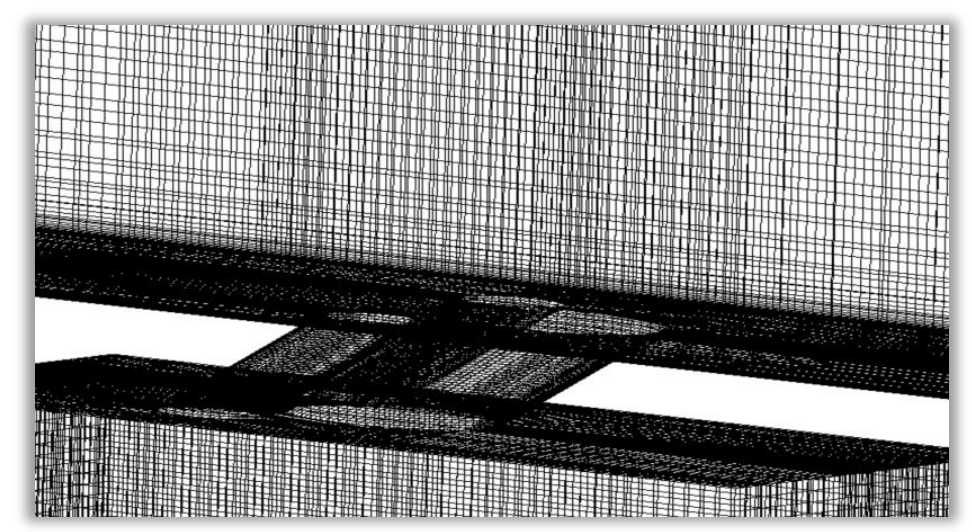

b) Isometric view is showing near holes mesh.

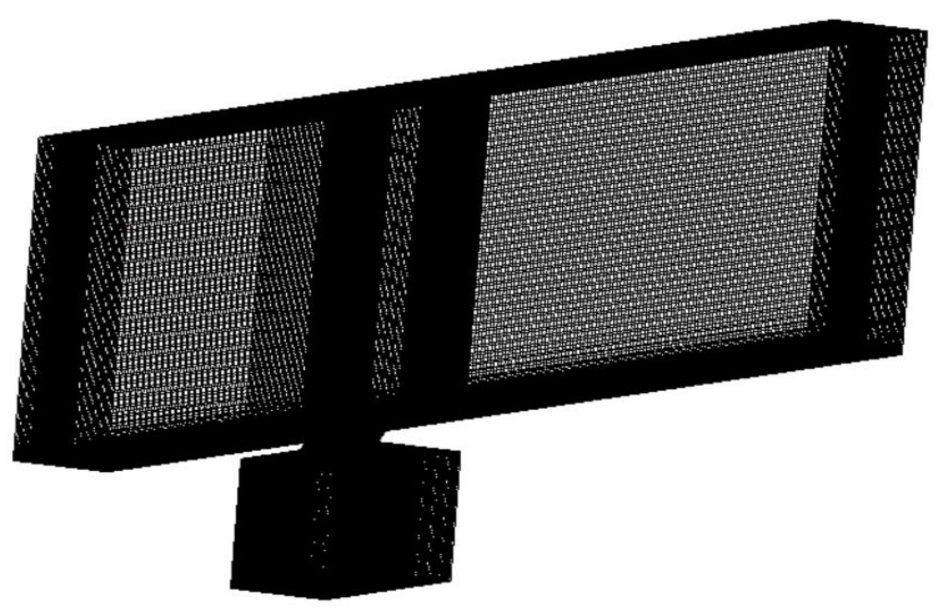

c) Isometric view.

Figure 6.4: Typical mesh generated for the $0^{\circ}$ (CAUSH) case. (Not to scale) 
All of the research efforts available on the open literature documented sister hole film cooling technique are performed numerically [61]-[69]; except the recent experimental study done by the research group of $\mathrm{Wu}$ et al. [10]. Also, all of those studies have examined sister holes that are only streamwise injected to the plate surface at $\alpha \approx 35^{\circ}$. Therefore, as a part of validation of the numerical results, the current computational results of the $0^{\circ}$ compound angle upstream sister holes are compared against the numerical results of Ely and Jubran [64], [65] for the upstream sister holes case; that is only streamwise injected at $\alpha=35^{\circ}$, at blowing ratio of $\mathrm{M}=0.5$ and D.R=1.2, as shown in Figure 6.6 a) and b). The centerline film cooling effectiveness results shown in Figure 6.6 a) illustrated that the current results pretty much agree well with the results of Ely and Jubran [65] for the short hole case ( $\mathrm{L} / \mathrm{D}=1.16)$ whereas the general trend is captured; however, a slight disagreement is seen at (X/D > 10). The difference becomes more pronounced between the current results and the results of Ely and Jubran [64] for long hole case, (L/D= 5.0), in the entire hole downstream domain. Several reasons could cause this difference in the predicted thermal-flow field parameters; (1) the variation in the cooling hole (L/D) ratio and the location of the sister holes in respect to the center of the main cooling hole, (2) the difference in the used coolant and freestream inlet boundary conditions and the turbulence boundary conditions. The current laterally averaged effectiveness results shown in Fig. 6.6 b) are consistent and represent a good agreement with Ely and Jubran [64], [65] in which the general trend is well captured.

\subsubsection{The Film Effectiveness Results of Compound Angle Upstream Sister Holes CAUSH}

The following cooling effectiveness numerical results of the CAUSH, both centerline and laterally averaged, are completed at a density ratio of 2 , and each CAUSH configuration is studied at four blowing ratios $\mathrm{M}=0.25,0.5,1.0$, and 1.5 . The primary results of the CAUSH film cooling performance are presented by Abd Alsalam and Jubran [101].

The centerline film cooling effectiveness results of all examined CAUSH at various blowing ratios; $M=0.25-1.5$, is presented in Figure 6.7 a)-d). Figure 6.7 a) in general shows that

at low blowing ratio, $M=0.25$, the centerline performance slowly decreases till it exhibits a plateau trend in the far downstream area of the hole exit for all studied CAUSH angles. 


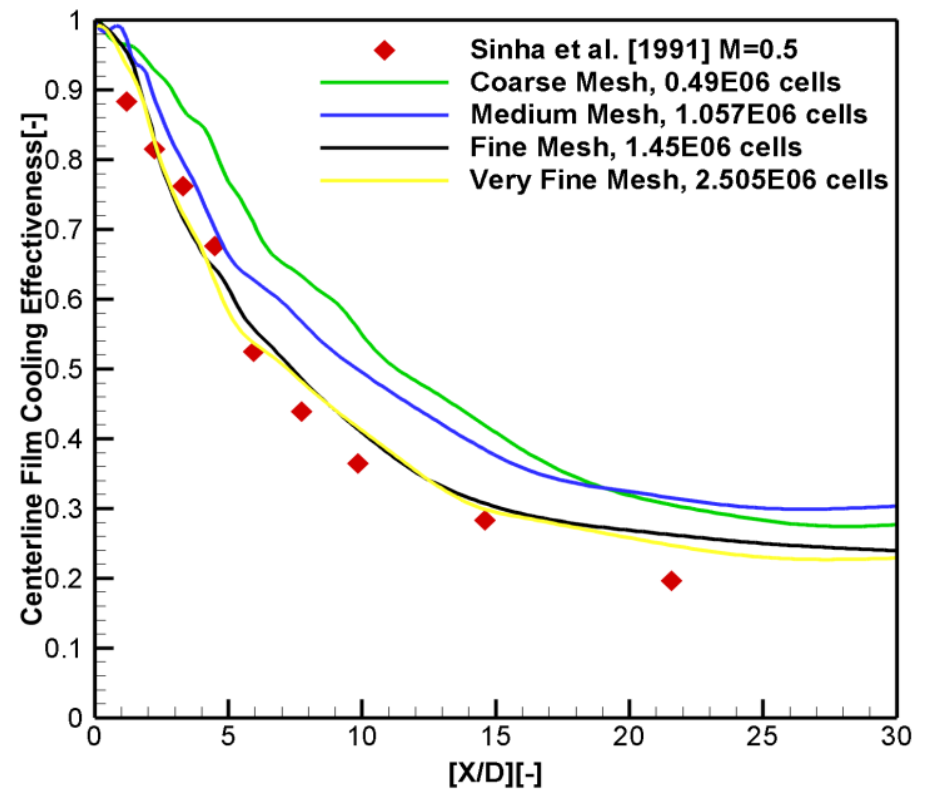

Figure 6.5: The grid sensitivity study and numerical results validation of centerline effectiveness at $\mathrm{M}=0.5$.

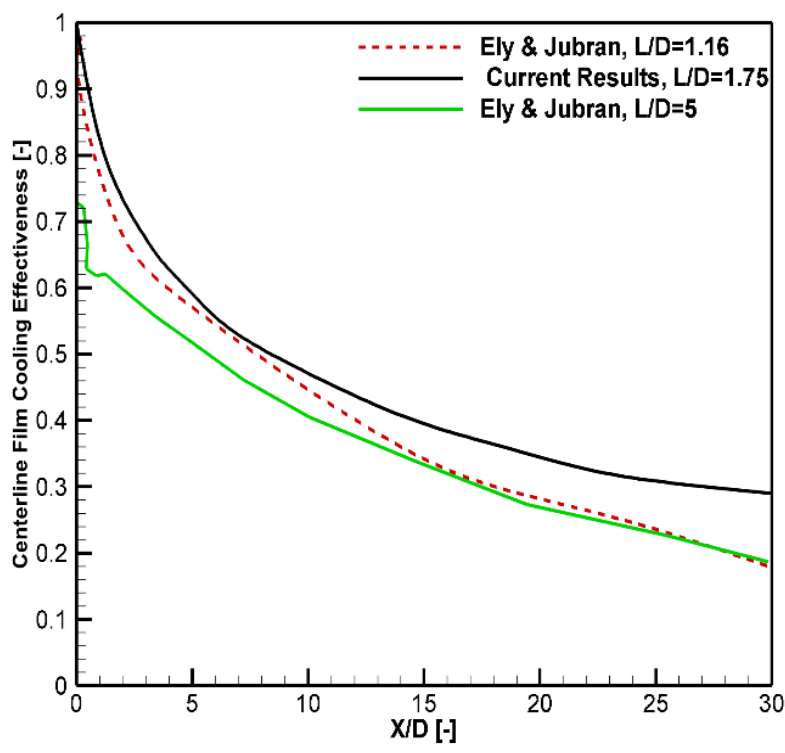

a) The centerline film cooling effectiveness.

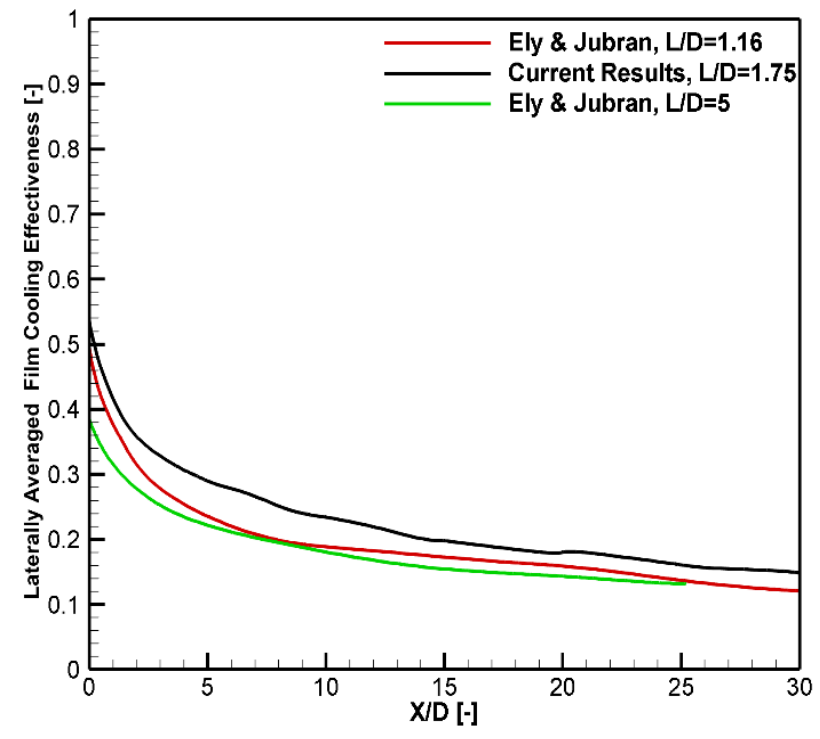

b) The laterally averaged film cooling effectiveness.

Figure 6.6: a) and b) The upstream sister holes centerline and lateral film effectiveness at $M=0.5$ \& D.R=1.2 compared to the CFD results of Ely and Jubran [64][65].

Furthermore, the centerline performance is almost the same for the $0^{\circ}, 45^{\circ}$, and $90^{\circ} \mathrm{CAUSH}$; however, the lowest performance for the entire downstream region is giving by $75^{\circ} \mathrm{CAUSH}$. It can be said that the sister holes with the compound angle at low blowing ratio $\mathrm{M}=0.25$ seems to 
be ineffective to improve the performance whereas the performance attained for all CAUSH angles has demonstrated no difference compared to $0^{\circ} \mathrm{CAUSH}$. The most likely cause for this low effect of compound angle on the sister holes centerline performance is stated by Li et al. [54], whereas they reported that compound angle of the short cooling hole did not exert any typical effect on the coolant jet in which the exit jet direction is nearly unchanged.

Increase the blowing ratio a little bit to $\mathrm{M}=0.5$, presents a significant increase in the centerline effectiveness for the entire downstream area in contrast to that accomplished at $\mathrm{M}=0.25$, as exhibited in Figure $6.7 \mathrm{~b}$ ). The maximum centerline effectiveness is found at $45^{\circ}$ CAUSH, followed by the performance of $0^{\circ}$ and $90^{\circ} \mathrm{CAUSH}$ in which the predicted centerline results at those two compound angles demonstrate a slight difference. Furthermore, the lowest performance is also shown at $75^{\circ} \mathrm{CAUSH}$. The reason for the enhancement in centerline performance of CAUSH at M= 0.5 is the attachment of coolant jet to the plate surface, which is improved further by the jet emerging out of the compound sister holes. Also, since the coolant jet has low momentum compared to the freestream, it gives the coolant favour and keeps it close to the plate surface with minimum mixing. Figure 6.8 shows the temperature contours at three spanwise planes; $\mathrm{X} / \mathrm{D}=0,1$, and 3 , and at the plate surface at blowing ratio $\mathrm{M}=0.5$. The plot demonstrates how the compound angle injection of sister holes changes the coolant attachment and distribution at both the plate surface and in the lateral direction. It is obvious from the plot that most of the compound angles keep the coolant significantly attached to the plate while low mixing and interaction are happing between the coolant and the freestream, as seen from the temperature contours. While the $45^{\circ} \mathrm{CAUSH}$ has shown the best coolant distribution at the plate surface, the $75^{\circ} \mathrm{CAUSH}$ has the worse as a result of the intensive mixing, and it seems no coolant coverage exists in the sister holes region.

Increasing the blowing ratio further above $\mathrm{M}=0.5$ had extremely changed and raised the centerline performance, whereby the benefit of orienting the sister holes into the freestream direction appears at high blowing ratios cases at $\mathrm{M}=1.0$ and $\mathrm{M}=1.5$, as depicted in Figure $6.7 \mathrm{c}$ ) and d). Figure $5.7 \mathrm{c}$ ) apparently indicates that; in contrast to $0^{\circ} \mathrm{CAUSH}$, the $90^{\circ}, 75^{\circ}$, and $45^{\circ}$ CAUSH revealed the ultimate centerline effectiveness for the whole downstream domain. 


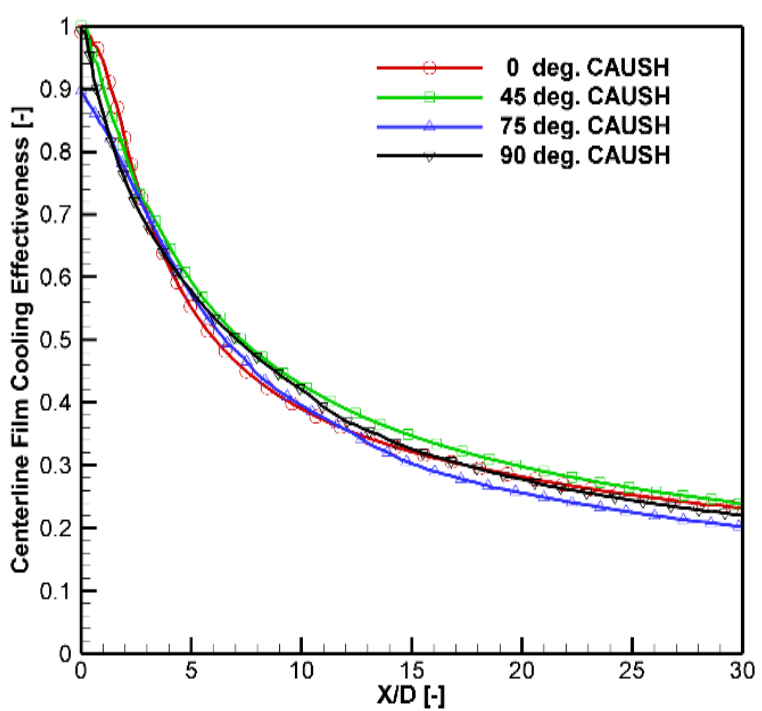

a) $\mathrm{M}=\mathbf{0 . 2 5}$

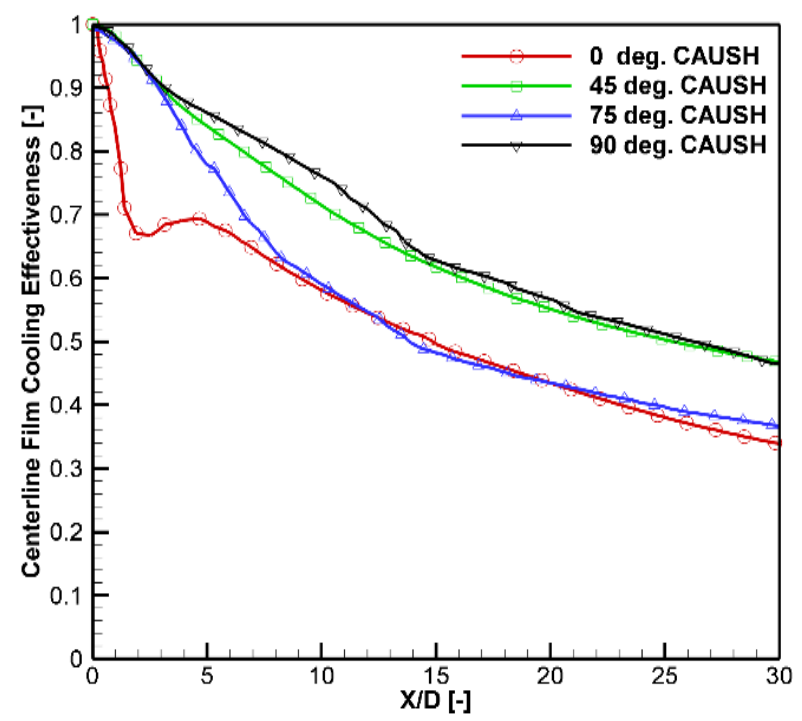

c) $M=1.0$

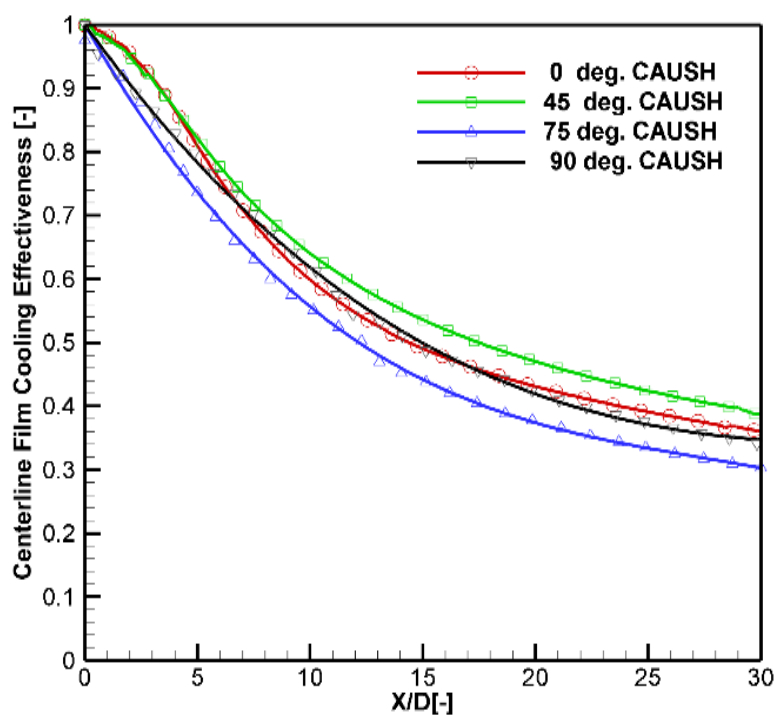

b) $M=0.5$

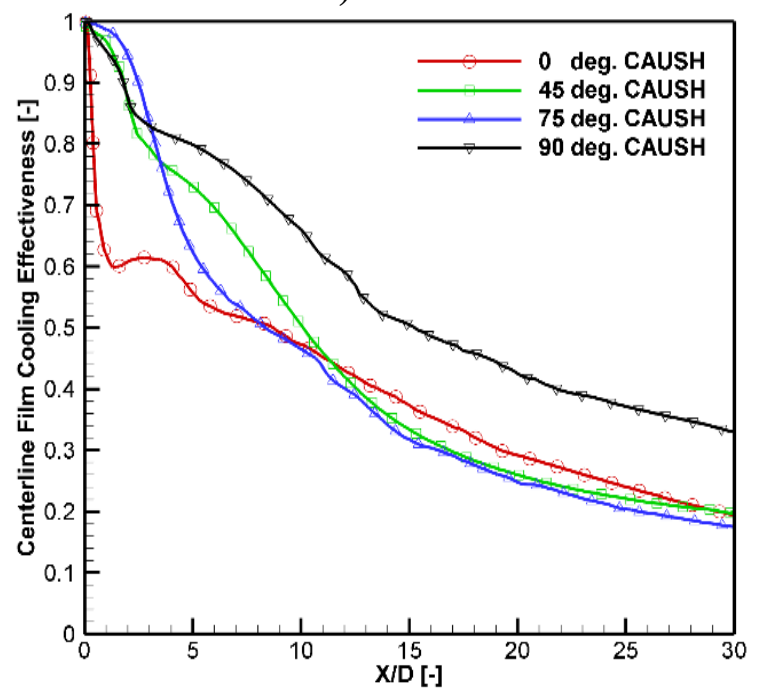

d) $M=1.5$

Figure 6.7: a) - (d the centerline film cooling effectiveness of CAUSH at different blowing ratios $\mathrm{M}=\mathbf{0 . 2 5}-1.5$.

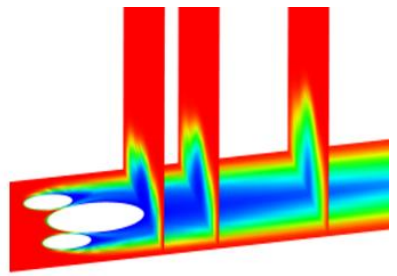

$0^{\circ} \mathrm{CAUSH}$

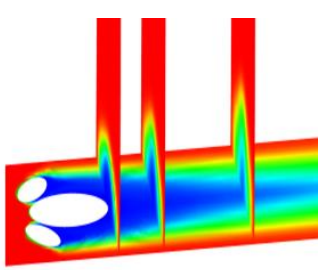

$45^{\circ} \mathrm{CAUSH}$

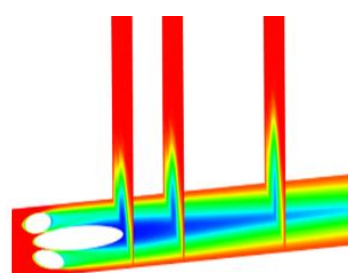

$75^{\circ} \mathrm{CAUSH}$

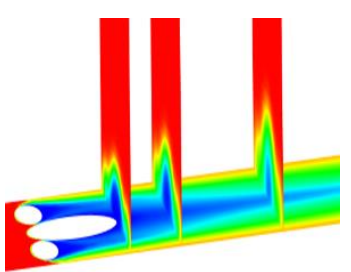

$90^{\circ} \mathrm{CAUSH}$

150160170180190200210220230240250260270280280300 Temperature K

Figure 6.8: temperature contours at three lateral planes; $X / D=0,1$, and 3 , and at the plate surface at $M=0.5$ 
However, the $75^{\circ} \mathrm{CAUSH}$ centerline performance gradually declines after (X/D > 5) to approach the $0^{\circ} \mathrm{CAUSH}$ performance. Figure $6.7 \mathrm{~d}$ ) shows the centreline effectiveness at the highest blowing ratio $\mathrm{M}=1.5$ for various CAUSH. It can be seen from the plot that the $75^{\circ} \mathrm{CAUSH}$ offers the most notable increase in the performance in the hole vicinity region up to $(\mathrm{X} / \mathrm{D} \leq 5)$. That exceeds the $45^{\circ}$ and $90^{\circ} \mathrm{CAUSH}$ effectiveness, whilst further downstream, its centerline effectiveness suddenly drops to give the minimum performance, nearly at $(X / D \geq 10)$. Moreover, $90^{\circ} \mathrm{CAUSH}$ case shows the best cooling effectiveness for most of the downstream area at (X/D $>5)$. $45^{\circ} \mathrm{CAUSH}$ offers good performance up to $(\mathrm{X} / \mathrm{D}<10)$, and then its performance is decreased further downstream. The predicted numerical results of centerline performance distributions at high blowing ratios $M=1.0$ and $M=1.5$, depicted in Figure $6.7 \mathrm{c}$ ) and d), has revealed that the detachment and the reattachment of the coolant jet happened in the near- hole region at $(2 \geq \mathrm{X} / \mathrm{D} \leq 5)$ for the $0^{\circ} \mathrm{CAUSH}$; whereas the sister holes injected only to the streamwise, does not happen for the compound angle injected sister holes. Therefore, this is an evident demonstrated that the compound angle injection of sister holes assists the coolant jet to remain fully attached to the plate surface at high blowing ratios. Thus, the compound angle sister holes film cooling technique can be considered a solution for one of the leading problems in film cooling; which is the coolant jet lift-off phenomena, and hence produced good thermal protection to the turbine blades and improved the overall engine performance.

The predicted results of the compound angle sister holes lateral effectiveness at various blowing ratios are shown in Figure 6.9 a) to d). In general, at low blowing ratios $\mathrm{M} \leq 0.5$, as depicted in Figure 6.9 a) and b), The laterally averaged performance of various CAUSH angle holes are almost the same, and a comparable trend has revealed. The $0^{\circ}$ and $90^{\circ} \mathrm{CAUSH}$ show the maximum lateral performance in the whole downstream region, while the lowest lateral effectiveness is obtained by $75^{\circ} \mathrm{CAUSH}$. At high blowing ratio the influence of the compound angle injection on sister holes lateral cooling performance is substantial and more pronounced, precisely in the hole adjacent region whereas the lateral performance is increased by increasing the compound angle, as presented in Fig $6.9 \mathrm{c}$ ) and d). In the meantime, further downstream at $(\mathrm{X} / \mathrm{D} \geq 5)$, the $45^{\circ} \mathrm{CAUSH}$ and the $75^{\circ} \mathrm{CAUSH}$ show a decrease in the lateral effectiveness even below the performance achieved by $0^{\circ} \mathrm{CAUSH}$. The lowest lateral 


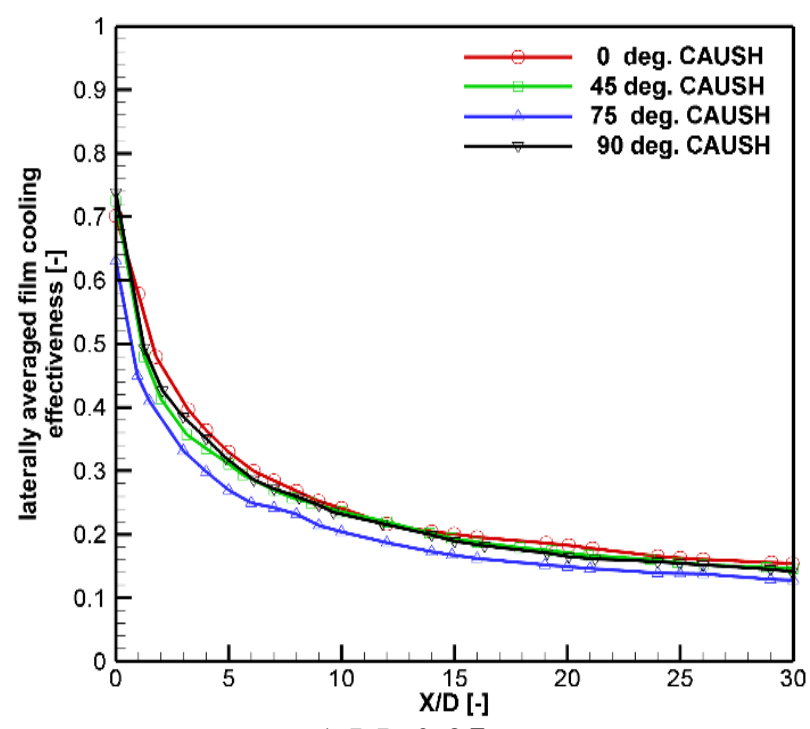

a) $M=0.25$

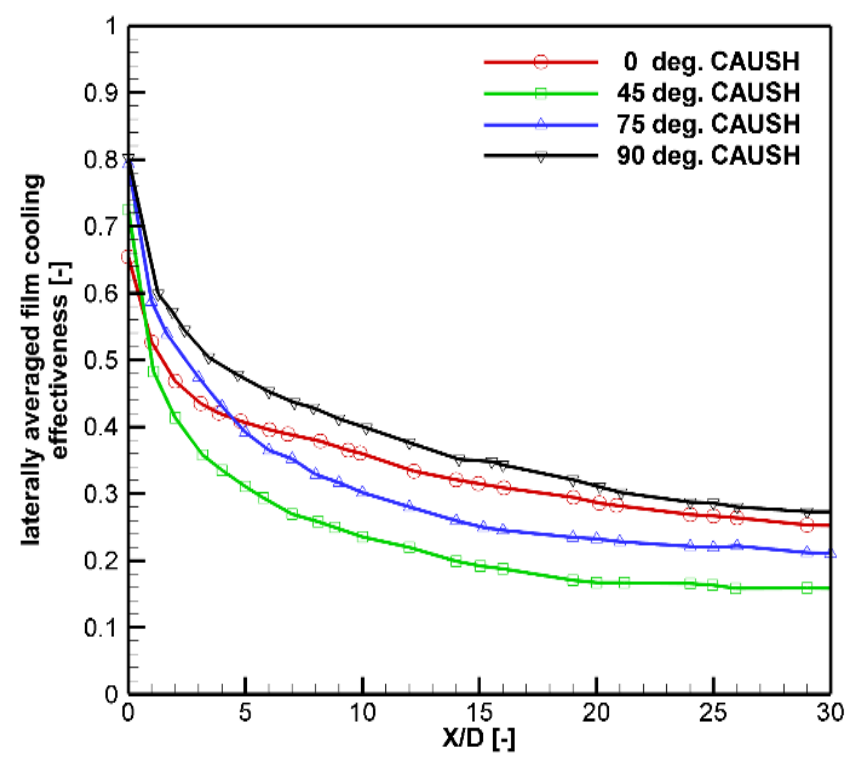

c) $M=1.0$

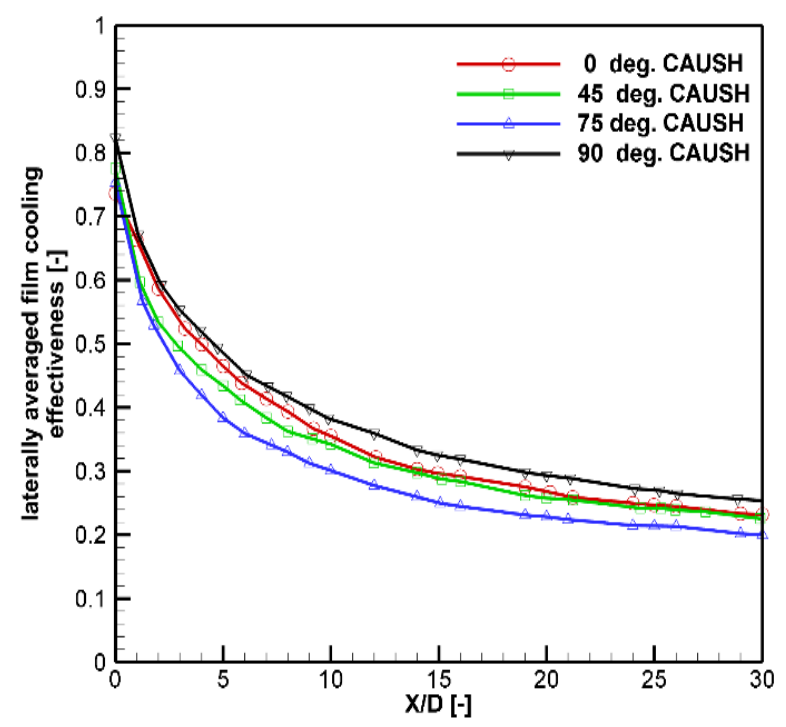

b) $M=0.5$

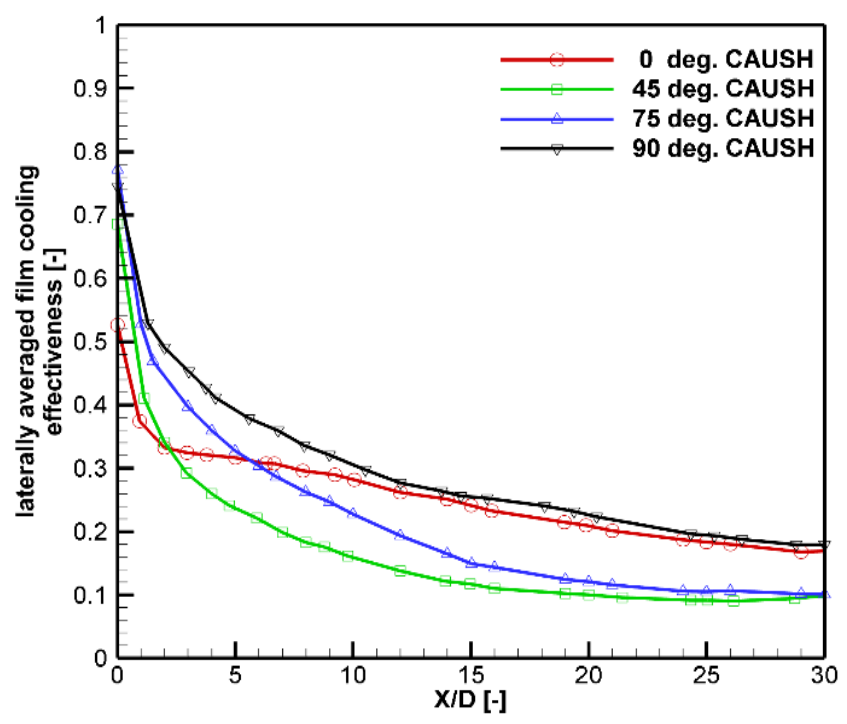

d) $M=1.5$

Figure 6.9: a) - (d The laterally averaged film cooling effectiveness of CAUSH at different

blowing ratios $\mathrm{M}=\mathbf{0 . 2 5}-\mathbf{1 . 5}$.

performance is obtained by $45^{\circ} \mathrm{CAUSH}$ amongst all other examined CAUSH in most of the downstream area, excluding the near hole downstream region at $(\mathrm{X} / \mathrm{D}<2)$.

It is well known in the literature that the compound angle of the single round cooling hole enhances the lateral effectiveness as a result of better coolant distribution spanwisely [54]. Consequently, it can be fairly stated that this analogy holds true for the CAUSH, whereas the coolant jet emerging from two compound sister holes is improving farther the coolant spreading 
and the uniform distribution of the coolant flow in the lateral direction. As such, compound angle upstream sister holes present a significant improvement in the centerline and lateral effectiveness for the whole downstream area.

The cooling performance of the compound angle upstream sister holes presented a distinguished variation on the centerline and lateral cooling performance for low and high blowing ratios cases for various examined CAUSH for the whole downstream area; precisely, the holes vicinity region. Therefore, to understand the main reasons of the attained performance and to assist how the CAUSH sustains the cooling jet adherent to the plate surface, the investigation of the local film cooling effectiveness contours at the plate surface will be given next at four blowing ratios; $M=0.25,0.5,1.0$, and 1.5, as shown in Fig. 6.10 and Fig. 6.11. The predicted results of local film cooling effectiveness at the plate surface are given in Figure 6.10 for low blowing ratios and Figure 6.11 for high blowing ratios on a $2 \mathrm{D}$ contours plot on $\mathrm{X}-\mathrm{Z}$ plane. The contours will be used as an indicator of the coolant coverage at the plate surface and to demonstrate the effect of the blowing ratio and the compound angle injection on the local effectiveness distributions. Overall, Figure 6.10 and Figure 6.11 noticeably exhibit that CAUSH presents an expanded and uniform coolant distribution in the streamwise and spanwise directions, and the distribution is roughly symmetry around $\mathrm{X}$-axis. Furthermore, CAUSH retains the coolant jet adhesion to the plate surface not only at low blowing ratios but also at the high blowing ratios, while a substantial coolant coverage is revealed. Increasing the compound angle effect on the local coolant distribution is prominent at high blowing ratios $\mathrm{M}=1.0$ and 1.5 compared to that at low blowing ratios $\mathrm{M}=0.25$ and 0.5 whereby the area of high local effectiveness concentration is extended further downstream the holes for the $45^{\circ}, 75^{\circ}$, and $90^{\circ} \mathrm{CAUSH}$ than the $0^{\circ} \mathrm{CAUSH}$. Figure $6.10 \mathrm{a}$ ) and b) presents that elevating the blowing ratio from 0.25 to 0.5 caused an increase in local effectiveness in the hole vicinity region whereas the high effectiveness region is expended from $\mathrm{X} / \mathrm{D} \leq 2.5$ to $\mathrm{X} / \mathrm{D} \leq 5$, whilst the improvement in the spanwise coverage is more distinct at $M=0.5$. Additionally, at low blowing ratios, increasing the compound angle of sister holes has an insignificant effect on the local effectiveness coverage, which is mainly limited to the hole vicinity region. Among all examined CAUSH holes, $75^{\circ} \mathrm{CAUSH}$ has the poorest coolant coverage where it appears that the coolant jet ejected out of the $75^{\circ} \mathrm{CAUSH}$ get mixed rapidly 

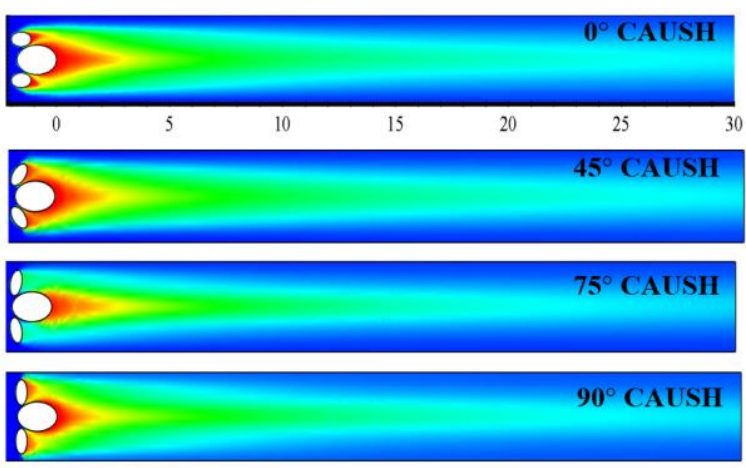

a) $\mathrm{M}=0.25$
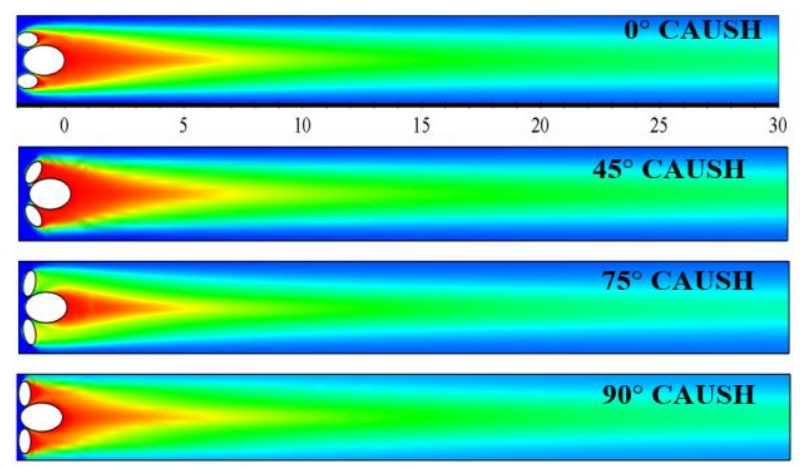

b) $\mathrm{M}=0.5$

$\begin{array}{llllllllllll}\text { Effectiveness } & 0 & 0.1 & 0.2 & 0.3 & 0.4 & 0.5 & 0.6 & 0.7 & 0.8 & 0.9 & 1\end{array} \longrightarrow \mathrm{x}$

Figure 6.10: The local film cooling effectiveness for various CAUSH at $M=0.25 \& 0.5$.

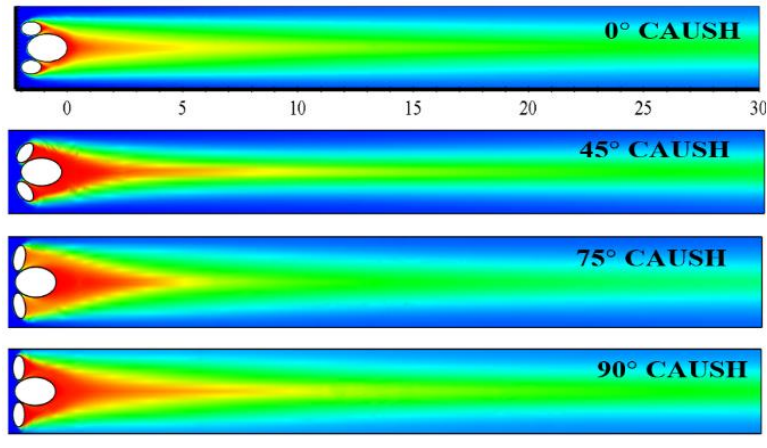

a) $\mathrm{M}=1.0$
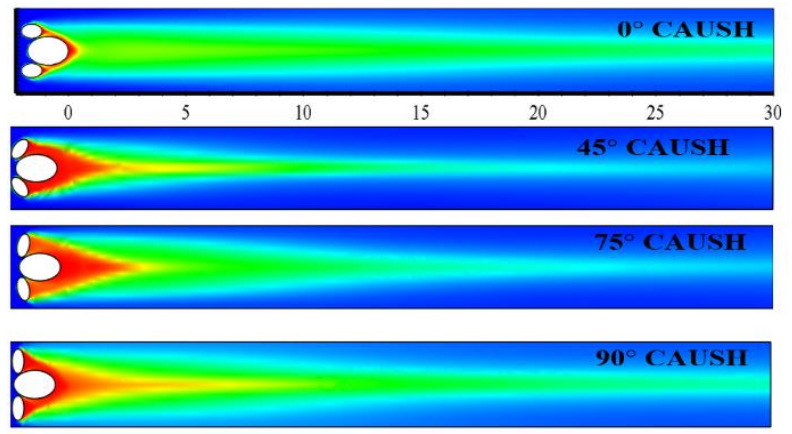

b) $\mathrm{M}=1.5$

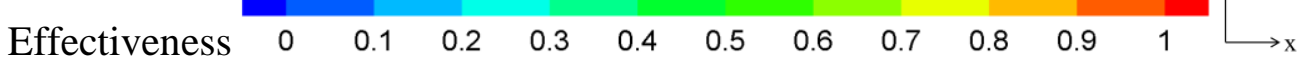

Figure 6.11: The local film cooling effectiveness for various CAUSH at $M=1.0 \& 1.5$.

with the mainstream. Consequently, in this case, the coolant concentration is restricted to the vicinity region of the primary hole. While further downstream, all the compound angle sister holes have nearly the same local coolant coverage. Figure 6.11 a) and b) exhibits that local effectiveness distribution improvement is obvious at blowing ratios $\mathrm{M}=1.0$ and 1.5 as well as the increase in the coolant coverage because of the coolant jet stays adherent to the plate surface. On the other hand, a fast deterioration of local effectiveness is experiential on the hole adjacent region at $\mathrm{M}=1.5$ for $0^{\circ} \mathrm{CAUSH}$ while a nominal coolant coverage is reached. This low performance of $0^{\circ} \mathrm{CAUSH}$ at $\mathrm{M}=1.5$ could be attributed to the intense mixing and penetration of the coolant jet into the freestream. The results of the local effectiveness of the $75^{\circ} \mathrm{CAUSH}$ demonstrates that by gradually increasing the blowing ratio from $\mathrm{M}=0.25$ to $\mathrm{M}=1.5$, the local coolant distribution and coverage is increased for the whole downstream area. Yet, it is smaller than that accomplished by $45^{\circ} \mathrm{CAUSH}$. Fig. $6.11 \mathrm{~b}$ ) shows that $75^{\circ} \mathrm{CAUSH}$ locally appears more effective and offers 
high local effectiveness mainly at X/D < 5; which is better than that obtained from the $90^{\circ}$ and $45^{\circ}$ (CAUSH). This could be attributed to the existence of the anti-counter rotating vortices pair that aided the coolant to be adherent to the surface with less mixing. Also, $75^{\circ} \mathrm{CAUSH}$ in the far downstream region has practically the same coolant coverage to the $45^{\circ} \mathrm{CAUSH}$. The optimum lateral, centerline coolant coverage and coolant uniformity for the whole downstream region for all examined blowing ratios are obtained by $90^{\circ} \mathrm{CAUSH}$. The outstanding cooling performance of the $90^{\circ}$ CAUSH could be related to the vorticities produced by $90^{\circ}$ that will be shown in the analysis of the coming results. In addition, When the coolant jet injected from both sister holes that are oriented to the mainstream direction by $\beta=90^{\circ}$, the elliptical exit shape of the holes will be parallel to the mainstream, and less mixing with the freestream is expected to occur. As such, sister holes injected jet supports the jet emerged from the main hole, resulting in a uniform full film coolant coverage.

Compound angle upstream sister holes previously discussed results herein of the centerline and lateral effectiveness revealed a significant variation in the cooling performance at low and high blowing ratios. As such, the coming results of the local lateral effectiveness will be shown at two blowing ratios; $M=0.5$ and $M=1.5$. Figure 6.12 a) and $b$ ) exhibits the local lateral cooling performance at four downstream positions $\mathrm{X} / \mathrm{D}=0,5,10$, and 15 at blowing ratios $\mathrm{M}=0.5$ and $\mathrm{M}=1.5$, respectively. Fig. $6.12 \mathrm{a}$ ) and b) reveals that the local lateral effectiveness distribution is symmetry around $\mathrm{Z} / \mathrm{D}=0$, which is the center of the lateral domain, and all the studied compound angle sister holes demonstrate a superior lateral spreading of the coolant at the plate surface. At $M=0.5$, Figure 6.12 a) shows that at $(X / D=0)$ all the examined CAUSH holes offer an excellent and identical lateral cooling performance in which all curves of the performance of various CAUSH holes collapse in one curve and the lateral effectiveness reached unity at the center and the vicinity region where $(Z / D \leq \pm 0.5)$. Moreover, the coolant spreading and lateral effectiveness slightly differ in the spanwise direction at $(Z / D> \pm 0.5)$, whereby the best lateral distribution is delivered by $90^{\circ}$ and $45^{\circ} \mathrm{CAUSH}$. When the sister hole is injected at $\beta=90^{\circ}$, the coolant jet gives the most significant coolant coverage, and it is widely distributed to cover the whole lateral distance of $(\mathrm{Z} / \mathrm{D}= \pm 1.5)$. On the other hand, the $0^{\circ}, 45^{\circ}, 75^{\circ} \mathrm{CAUSH}$ local lateral performance is decreased to reach zero at nearly $(\mathrm{Z} / \mathrm{D}= \pm 1.45)$. By moving further downstream at $\mathrm{M}=0.5 ; \mathrm{X} / \mathrm{D}=5,10$, and 15 , all the examined $\mathrm{CAUSH}$ have 
a) $\mathrm{M}=0.5$
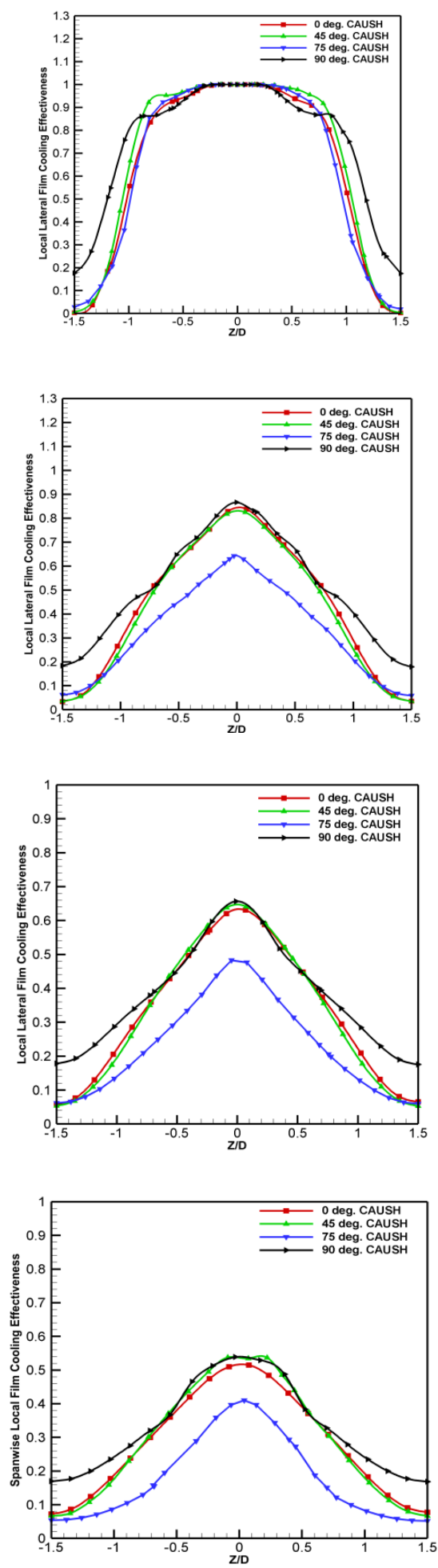

b) $M=1.5$

$\mathrm{X} / \mathrm{D}=\mathbf{0 . 0}$

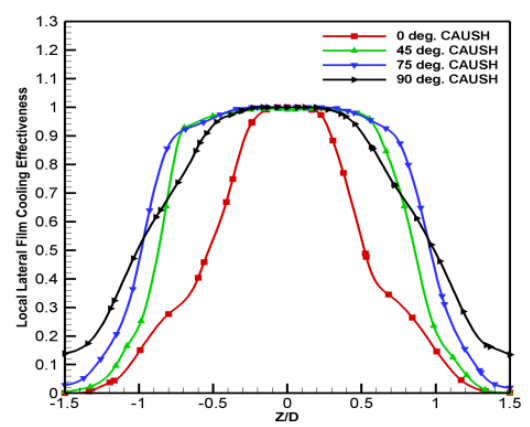

$\mathrm{X} / \mathrm{D}=\mathbf{5 . 0}$

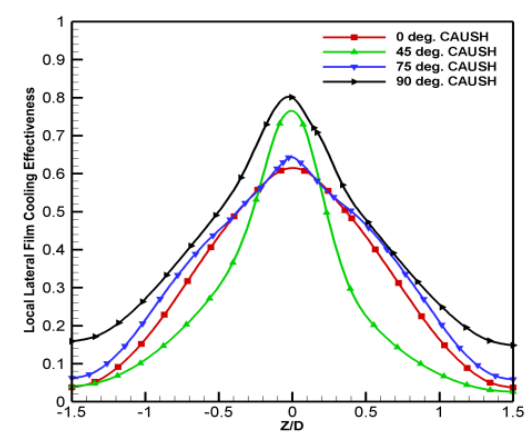

$\mathrm{X} / \mathrm{D}=\mathbf{1 0 . 0}$

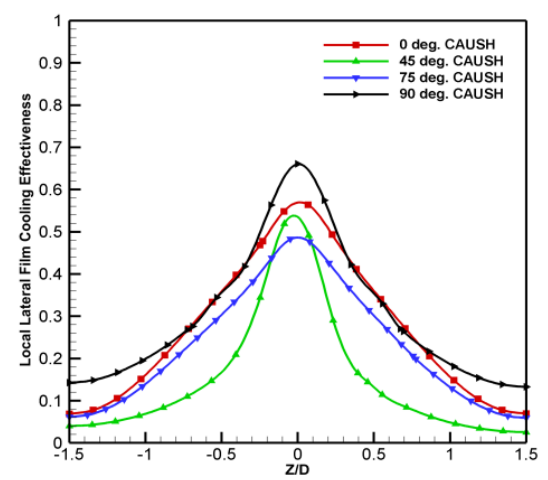

$\mathrm{X} / \mathrm{D}=\mathbf{1 5 . 0}$

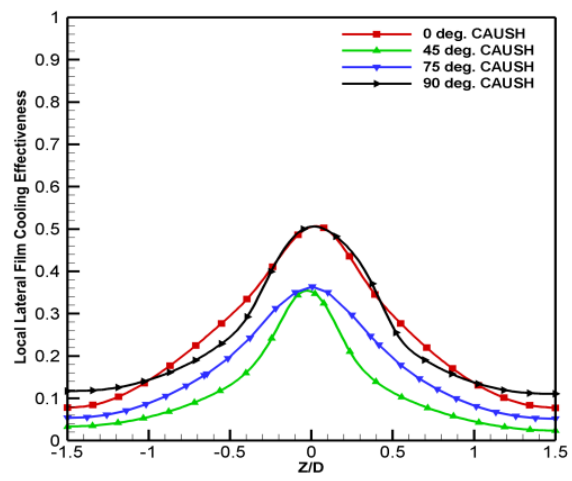

Figure 6.12: Local lateral film effectiveness for various CAUSH at a) $M=0.5 \&$ b) $M=1.5$. 
shown that the peak of the lateral local effectiveness is gradually decreased whereas the $90^{\circ}$ CAUSH still offers the optimum performance for the entire lateral domain, while its performance is similar to the $0^{\circ}$ and $45^{\circ} \mathrm{CAUSH}$ close to the center and its vicinity region. On the other hand , $75^{\circ} \mathrm{CAUSH}$ offers the lowest performance amongst all $\mathrm{CAUSH}$ while the $0^{\circ}$ and $45^{\circ} \mathrm{CAUSH}$ lateral performance drop close to the lateral domain boundary and found to be similar to that of $75^{\circ} \mathrm{CAUSH}$. This deviation of the local lateral effectiveness amongst the examined CAUSH away from the center of the lateral domain might be attributed to the variation in the mixing and interaction of the coolant jet and the freestream. At high blowing ratio $\mathrm{M}=1.5$, Figure $6.12 \mathrm{~b}$ ) presents that at $(\mathrm{Z} / \mathrm{D}=0)$ the $90^{\circ}, 45^{\circ}, 75^{\circ} \mathrm{CAUSH}$ still show notable coolant spreading and local lateral effectiveness which is nearly similar to that attained at $\mathrm{M}=0.5$; while the noticed difference is in the local effectiveness values close to ( $\mathrm{Z} / \mathrm{D} \approx \pm 1.5$ ), which is lower for the $\mathrm{M}=1.5$ for all CAUSH. Also, the $0^{\circ} \mathrm{CAUSH}$ shows the lowest coolant spreading and local lateral effectiveness, whereas its cooling performance significantly drops after $(\mathrm{Z} / \mathrm{D} \approx \pm 0.35)$ in contrast to other examined CAUSH. It can be said that the compound angle sister holes and regardless of the downstream location, dominates a remarkable local lateral performance and coolant jet attachment to the plate surface. The most effective CAUSH performance is attained by $90^{\circ}$ CAUSH, and the effectiveness level decreases by moving further downstream whereby according to Jung and Lee increasing the orientation angle $\beta$ leads to an increase in the spanwise component of coolant jet momentum, which benefits the spanwise coolant coverage and the coolant uniformity [104].

It is significantly critical to associate the outstanding performance of the CAUSH to that of a single cylindrical hole simply injected to the streamwise because CAUSH requires one and a half more coolants than that of the single hole at the same blowing ratio. Moreover, to ensure that the improvement in the cooling performance is sufficiently high to compensate for the additional amount of the used coolant jet. Consequently, the proposed novel CAUSH overall film cooling effectiveness is compared to that of the single cylindrical hole at various blowing ratio $\mathrm{M}=0.25-1.5$, as depicted in Figure 6.13. The plot obviously shows that the overall cooling effectiveness of all examined CAUSH is continually increased by increasing the blowing ratio; however, the round hole best cooling performance is attained at $\mathrm{M}=0.5$, and after that, a sharp deterioration in the performance is observed. All CAUSH holes have their best performance at $\mathrm{M}=1.0$, and after that, a slight reduction in the performance appears at $M=1.5$. The optimal overall 
performance is accomplished by $90^{\circ} \mathrm{CAUSH}$ for all blowing ratios, whilst at $\mathrm{M}=0.25$ all studied CAUSH holes present almost the same performance. Contrary to the single cylindrical hole overall film cooling effectiveness, compound angle upstream sister holes have given a substantial improvement in the overall cooling performance in which it is magnified by a factor of 2.0, 1.97, 1.91, and 2.26 for the $0^{\circ}, 45^{\circ}, 75^{\circ}$, and $90^{\circ} \mathrm{CAUSH}$, respectively [101]. Thus, it is apparent that the gain in cooling performance is substantial enough to justify the additional usage of coolant.

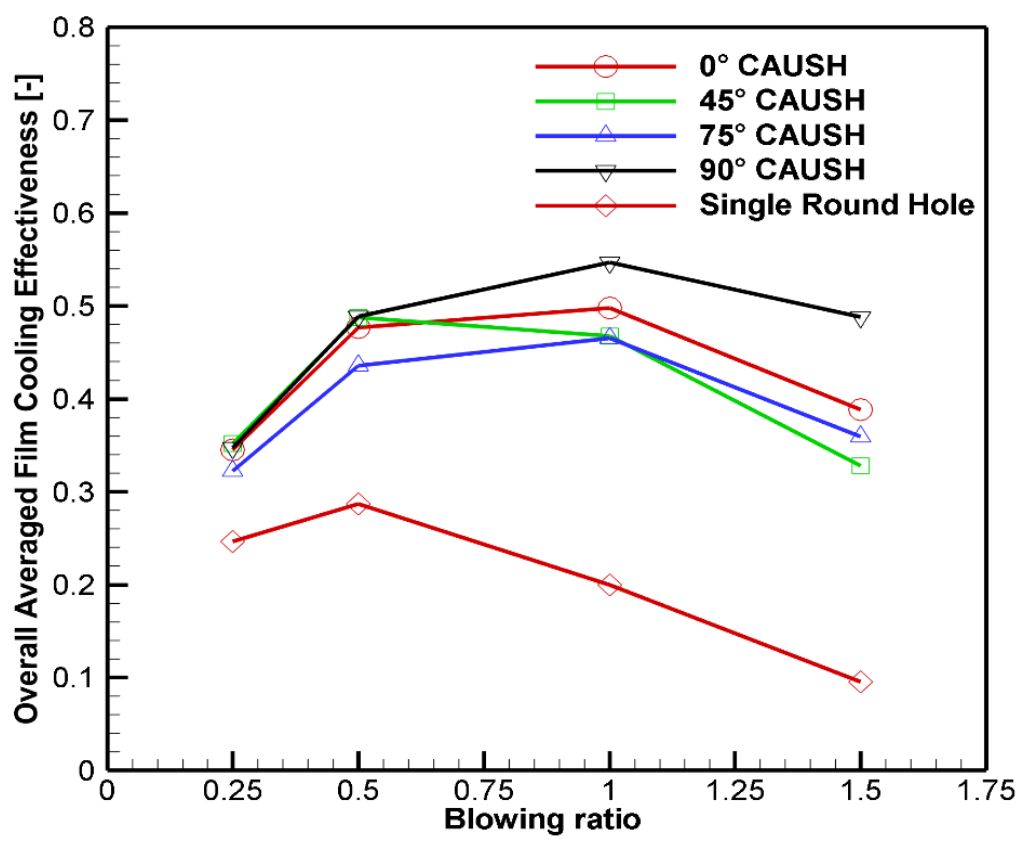

Figure 6.13: A Comparison of the overall film cooling effectiveness of (CAUSH) to that of a single cylindrical hole at $\mathrm{M}=0.25-1.5$.

\subsubsection{Flow Structure of Compound Angle Upstream Sister Holes}

The vortices structure appears in a counter-rotating vortices pair CRVP which occurs from the interaction of the cooling jet with the freestream for a single cylindrical hole has an undesirable effect on cooling performance and coolant jet attachment at high blowing ratio [45]. This flow structure is significantly different for sister holes cooling technique, whereas sister holes produce an anti-counter rotating vortices pair as documented by Ely and Jubran[61]-[63], [65]. Therefore, to understand the dominant flow field of the compound angle upstream sister holes that is responsible for its thermal performance, the predicted results for 

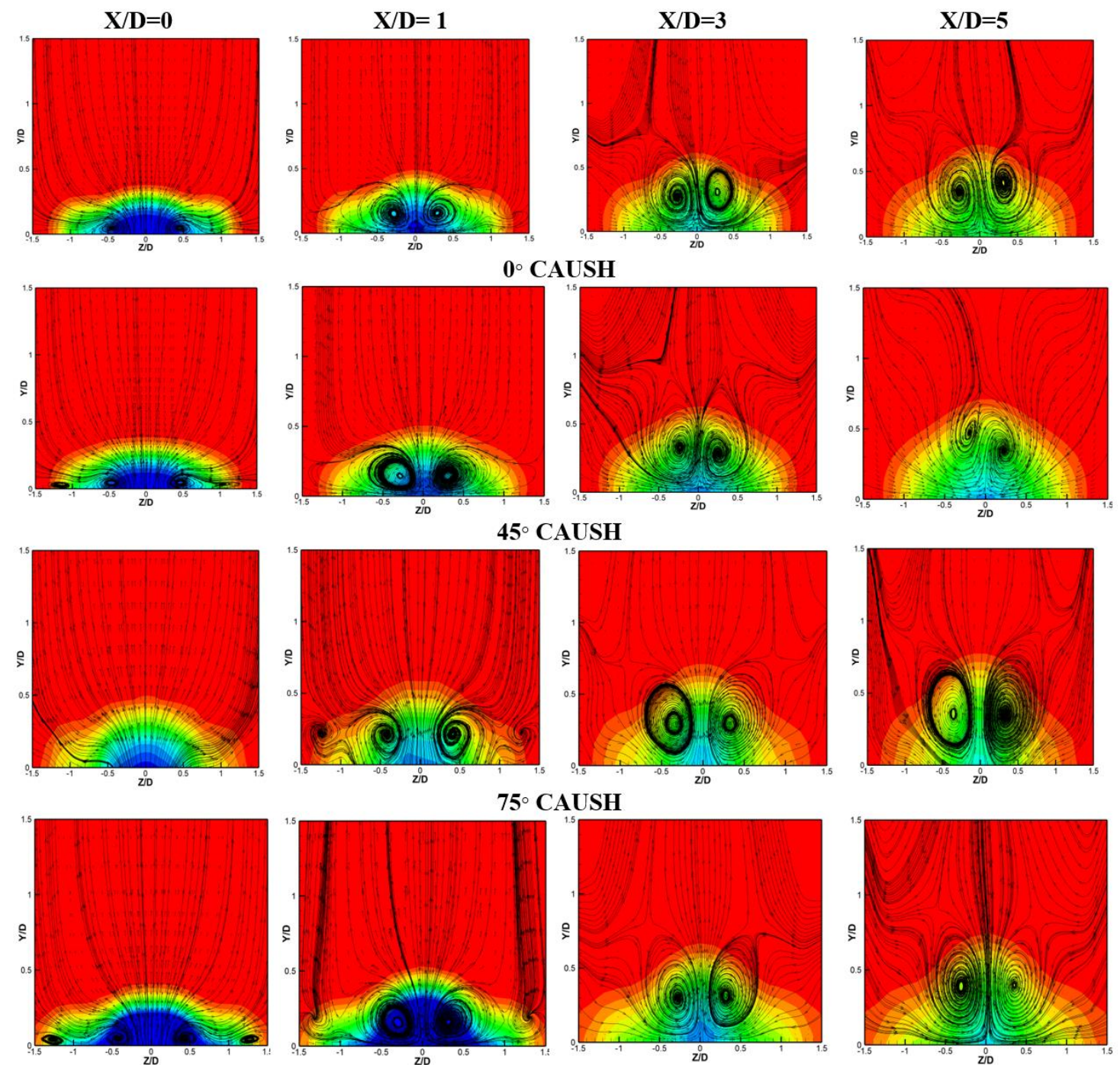

90 ${ }^{\circ}$ CAUSH

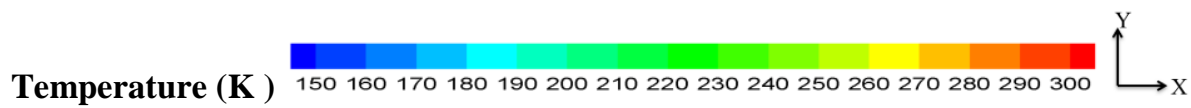

Figure 6.14: Flow structure of the examined CAUSH at $M=0.25$ and $X / D=0,1,3$, and 5 .

the CAUSH flow field and vorticial structures development downstream of the cooling hole is presented and discussed at various blowing ratios $\mathrm{M}=0.25-1.5$ at four downstream positions in the hole vicinity region; $X / D=0,1,3$, and 5 . The 2D flow structure results of CAUSH will be presented in a $\mathrm{YZ}$ plane perpendicular to the plate surface, which is extended 1.5D horizontally in the $\mathrm{Z}$-axis to both sides from the centerline and $1.5 \mathrm{D}$ vertically in the $\mathrm{Y}$-axis. Streamlines and velocity vectors are used to specify the vortices rotation direction, strength, and size. The temperature contours are used to indicate the mixing rate of the coolant with the freestream. The 
flow structure results for low blowing ratios; $M=0.25$ and $M=0.5$, are depicted in Figures 6.14 and 6.15, respectively. Also, the flow structure results for high blowing ratios, $M=1.0$ and $M=1.5$, are shown in Figures 6.16 and Figure 6.17, respectively.

At low blowing ratio, $\mathrm{M}=0.25$, Fig. 6.14 exhibits that the coolant jet core is attached to the plate surface for all CAUSH in the whole-vicinity region $(X / D=0-5)$. This is due to the low coolant jet momentum and the less penetration of the coolant jet into the freestream. Also, jet lift-off has not occurred at this blow ratio. But the $0^{\circ} \mathrm{CAUSH}$ at $\mathrm{X} / \mathrm{D}=5$ presents that the coolant core is apparently dissipated because of the intense mixing, as depicted by temperature contour. In terms of the vortices structure, Figure 6.15 at $\mathrm{X} / \mathrm{D}=0$, shows the generation of the anti-counter rotating vortices pairs ACRVP very close to the plate surface, whereby its center is nearly located at $( \pm 1.05,0.04)$ for $0^{\circ}, 45^{\circ}$, and $75^{\circ} \mathrm{CAUSH}$. The generated ACRVP tends to reduce the strength of the CRVP of the primary cooling hole where its center is located at $( \pm 0.45$, 0.06). Also, the coolant concentration is symmetrically distributed around Z-axis for $0^{\circ}, 45^{\circ}$, and $75^{\circ} \mathrm{CAUSH}$ to covers the domain of $( \pm 0.8,0.2)$. While the $90^{\circ} \mathrm{CAUSH}$ covers the area of $( \pm 1.1$, $0.24)$, and its ACRVP is located far-off from each other at $( \pm 1.25,0.04)$; which aids the coolant jet to widely spreading in the lateral direction amongst other CAUSH. At $\mathrm{X} / \mathrm{D}=1$ and for all CAUSH holes, the ACRVP and CRVP are developed and become a little bit stronger compared to that at $X / D=0$ while they shifted up in the vertical direction far from the surface and their centers are located at $( \pm 1.3,0.22)$ and $( \pm 0.45,0.19)$, respectively. Moreover, interestingly, at X/D $=1$, the $0^{\circ}$ and $45^{\circ} \mathrm{CAUSH}$ present that the ACRVP meet and emerge with the outer boundary of the CRVP while there is a strong downwash vortex tends to maintain the coolant adherent to the plate surface. This unique flow structure for $0^{\circ}$ and $45^{\circ} \mathrm{CAUSH}$ at $\mathrm{M}=0.25$ might be the cause of achieving the maximum centerline and lateral performance in the hole vicinity region at $(\mathrm{X} / \mathrm{D}=1)$. However, the $75^{\circ} \mathrm{CAUSH}$ at $\mathrm{X} / \mathrm{D}=1$ exhibits more mixing in the vertical and lateral direction whereas the coolant coverage and its core is notably less than that of other CAUSH resulting in the lowest performance of $75^{\circ} \mathrm{CAUSH}$. At X/D $=3$ and 5, all CAUSH show that the ACRVP continue to develop farther and become stronger, while CRVP starts to be less notable. Yet, its vestiges can still be recognized that caused a slight drop in the performance.

Figure 6.15 shows the predicted flow structure results at $M=0.5$. The plot clearly shows that the coolant core is attached to the plate surface for all examined CAUSH, not only at X/D 

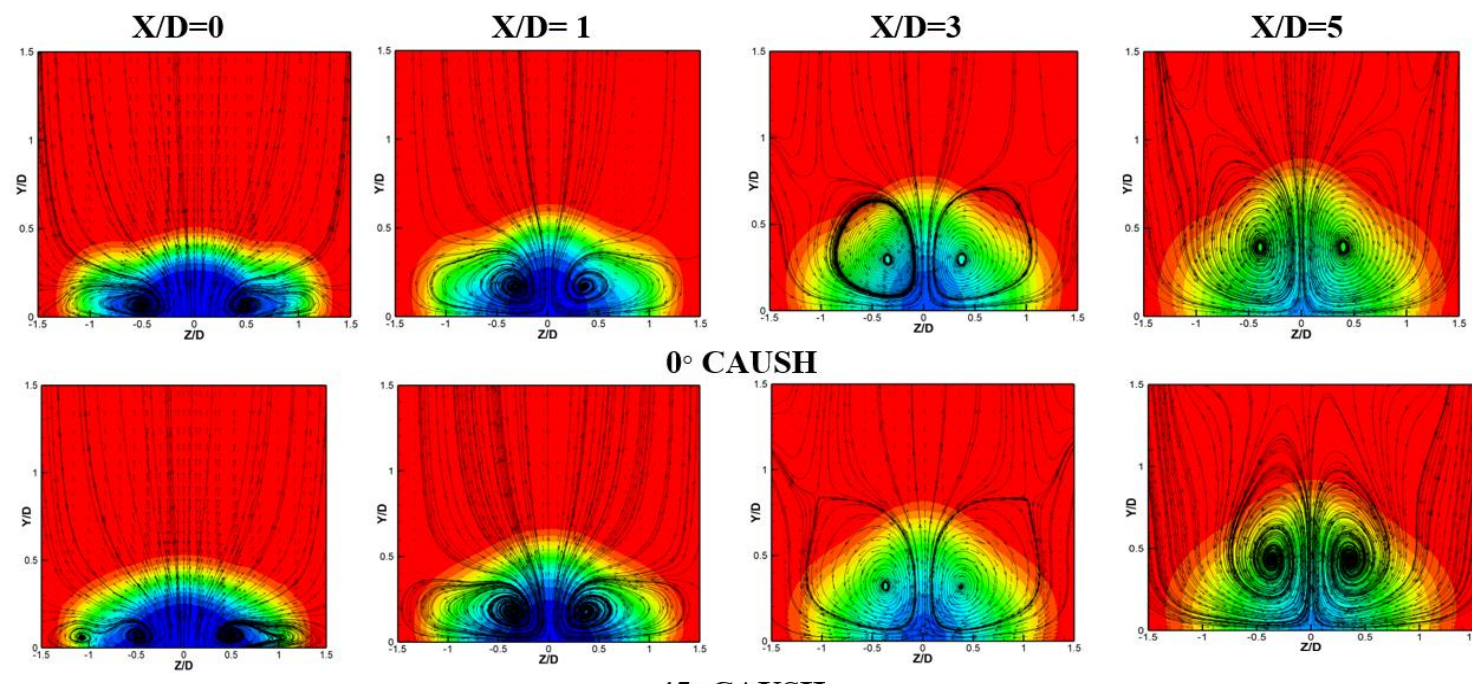

0 ${ }^{\text {CAUSH }}$
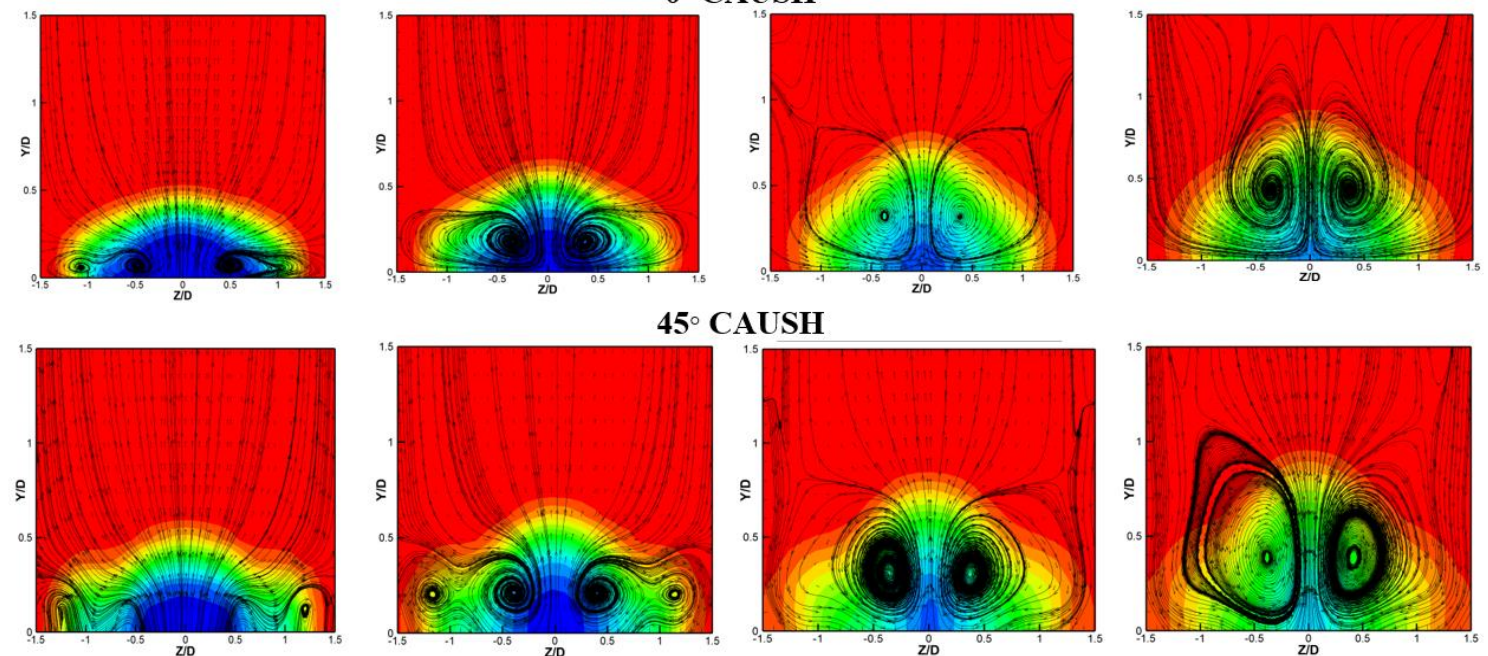

$45^{\circ}$ CAUSH
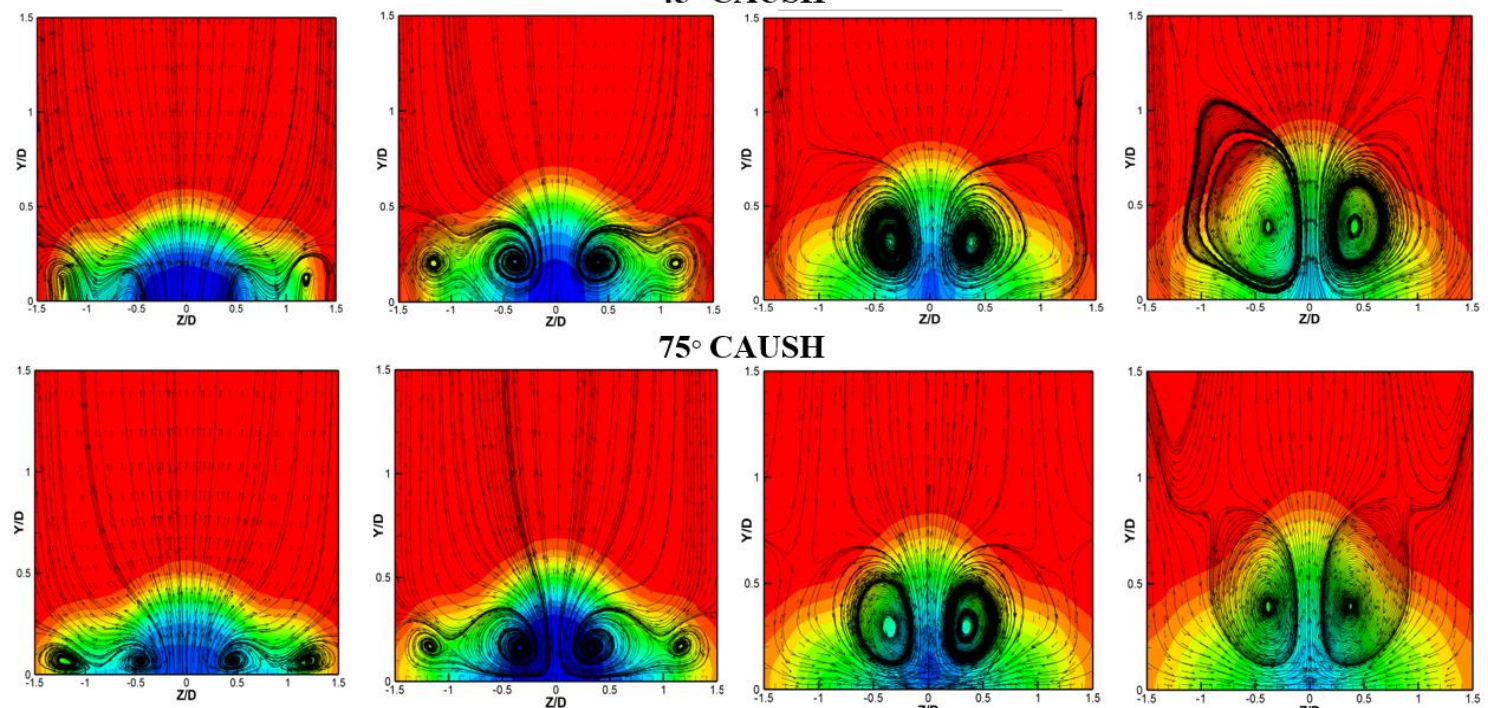

$5^{\circ}$ CAUSH
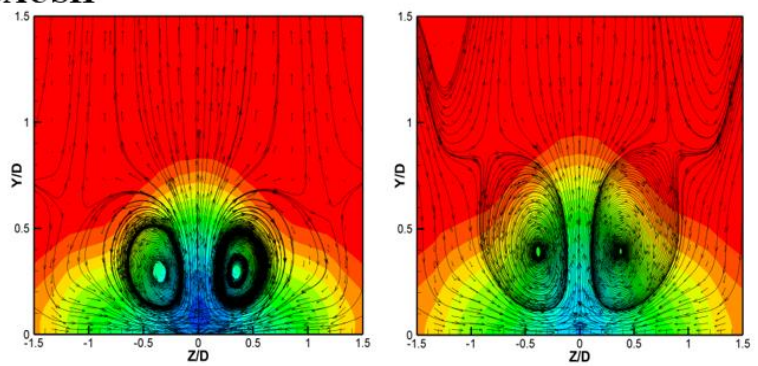

90 ${ }^{\circ}$ CAUSH

Temperature (K) $150160170180190200210220230240250260270280290300 \longleftrightarrow x$

Figure 6.15: Flow structure of the examined $\mathrm{CAUSH}$ at $\mathrm{M}=0.5$ and $\mathrm{X} / \mathrm{D}=0,1,3$, and 5 . $=0$ but also at $\mathrm{X} / \mathrm{D}=5$ as an indication of no coolant lift-off happened. The coolant core is exhibited as a single integrated jet concentrated in the center of the computational domain, and it is expanded more in the lateral and the vertical directions to cover a larger area with less mixing and interaction with the mainstream compared to that reached at $M=0.25$. Moreover, Figure 6.15 presents that all examined CAUSH at X/D $=0$ and 1, display the existence of ACRVP which is obviously larger or in a similar size as the CRVP for the primary hole. As such, the ACRVP becomes further effective to diminish the CRVP effect as the compound angle is increased. Thus, 
it enhances the coolant spreading, coverage, and uniformity in the lateral direction. The centers of ACRVP and CRVP can be seen approximately located at $( \pm 1.2,0.19)$ and $( \pm 0.35,0.19)$, respectively. At $\mathrm{X} / \mathrm{D}=1,0^{\circ}$ and $45^{\circ} \mathrm{CAUSH}$ demonstrate a similar trend for the flow structure and coolant coverage whereas the ACRVP is kind of emerging with the CRVP, and this caused a vertical growth to the coolant core while the attachment region of the coolant in the lateral direction is somewhat reduced along with the lateral coolant coverage. Interestingly, $75^{\circ} \mathrm{CAUSH}$ at X/D = 1 shows a strong CRVP at an equivalent vertical level of the ACRVP which caused a contraction of lateral coolant distribution and coolant core among other examined CAUSH while more mixing appears, as depicted by the temperature contour. The $90^{\circ} \mathrm{CAUSH}$ case at X/D=1 exhibit the largest area of coolant jet attachment and the lateral coolant distribution, while a weak CRVP appears generated from the primary hole. Ultimately, ACRVP works effectively to decrease the upwash vorticity of the main hole and maintaining the coolant jet full adhesion to the plate surface. Also, the coolant distribution mostly seems to cover the whole lateral domain; $(\mathrm{Z} / \mathrm{D}= \pm 1.5)$. This explains why the $90^{\circ} \mathrm{CAUSH}$ offered the maximum lateral performance at $\mathrm{M}=0.5$ in the adjacent region of the hole. Additionally, Figure 6.15 at $\mathrm{X} / \mathrm{D}=3$ and 5 , illustrates that for all studied CAUSH, the coolant core is attached to the surface due to prominently displayed ACRVP; however, more mixing is shown, which leads to a decrease on the performance. Ely and Jubran [64], [65], as well as Wu et al. [10], found the same flow structure results of $0^{\circ} \mathrm{CAUSH}$ at $\mathrm{M}=0.5$ whereas they reported the ACRVP produced by sister holes is efficiently controlling the CRVP of the main hole.

It is very interesting to reveal that for high blowing ratios $M=1.0$ and $M=1.5$, as depicted in Figure 6.16 and figure 6.17; the coolant core remains significantly attached to the plate surface at $(\mathrm{X} / \mathrm{D}=0$ to 5$)$. Also, a substantial coolant spreading in the lateral and vertical directions is noticed, which is an evidence to the superior benefit of the employment of the CAUSH to sustain the coolant adhesion to the surface while providing full protection to the plate surface. Figure 6.16 and figure 6.17 demonstrates that all $\mathrm{CAUSH}$ at $\mathrm{X} / \mathrm{D}=0$ offers widespread lateral coolant attachment region. However, this region is contracted, and it expands vertically further downstream of the hole exit at $X / D=1,3$ and 5. At the same time, the ACRVP is noticeably effective in controlling the CRVP, whereas the ACRVP are shifted upward in the y-direction, which leads to a vertical expansion of the coolant while it diminishes the CRVP. To explain it further, the $90^{\circ} \mathrm{CAUSH}$ at $\mathrm{M}=1.0$ is used as an example. Figure 6.16 demonstrates 

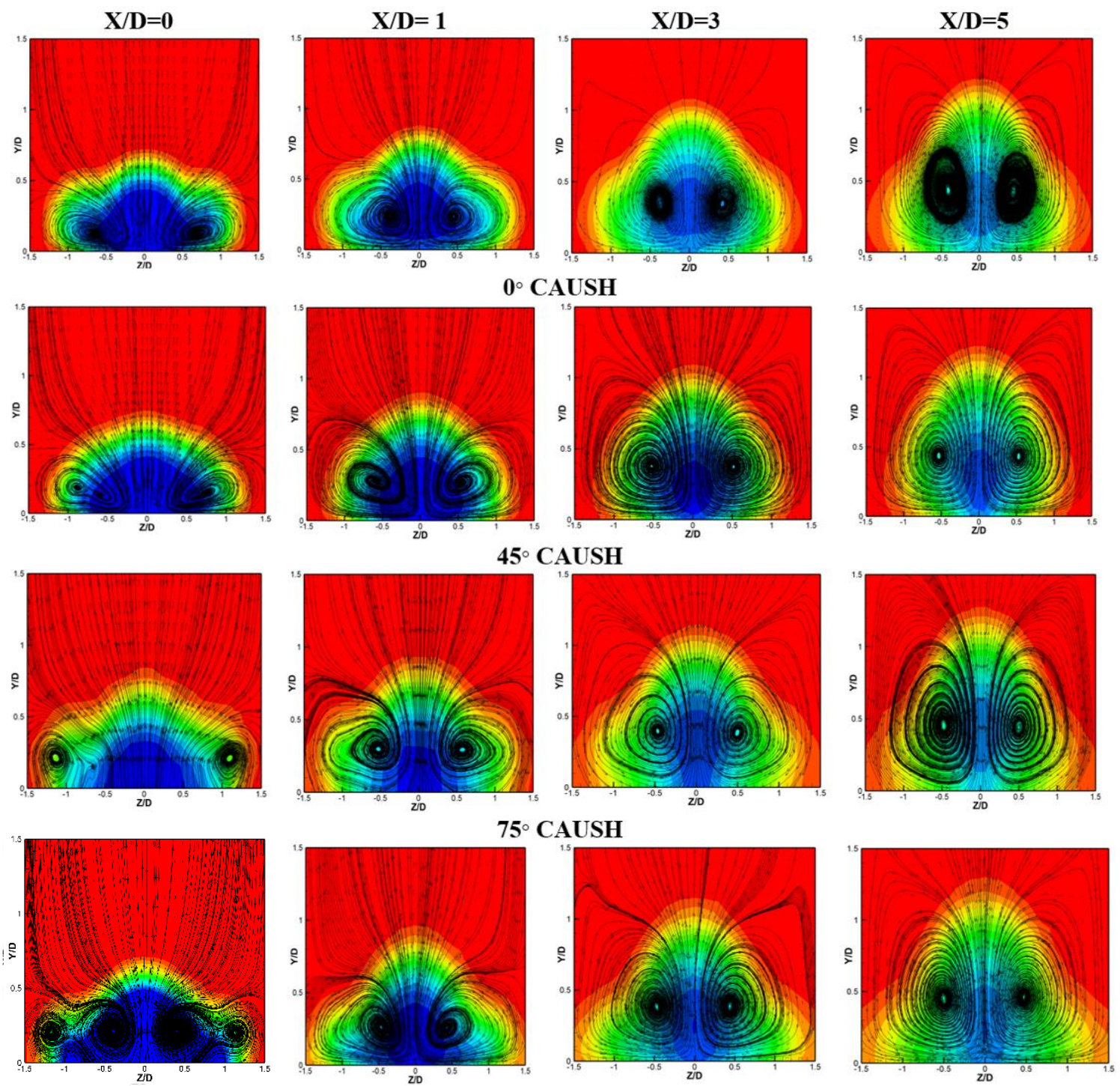

90 ${ }^{\circ}$ CAUSH

Temperature (K ) $150160170180190200210220230240250260270280290300 \stackrel{x}{\longrightarrow}$.

Figure 6.16: Flow structure of the examined CAUSH at $M=1.0$ and $X / D=0,1,3$, and 5.

that $90^{\circ} \mathrm{CAUSH}$ yields the optimal coolant coverage and attachment at $\mathrm{X} / \mathrm{D}=0$ whereby the coolant jet covers the computational domain area of $( \pm 1.1,0.5)$, while the center of the CRVP and the ACRVP is located at $( \pm 0.5,0.12)$ and $( \pm 1.1,0.14)$, respectively. This spanwise location of the ACRVP; at $\mathrm{Z} / \mathrm{D}= \pm 1.1$ close to the domain boundary, makes it effective to suppress the CRVP of the main hole. Moreover, $90^{\circ} \mathrm{CAUSH}$ at $\mathrm{X} / \mathrm{D}=3$ and 5 shows that ACRVP is centred at $( \pm 0.48,0.38)$ and $( \pm 0.48,0.44)$, respectively, whereby it shows a strong downwash vortex that roles the coolant toward the plate surface and avoids the freestream to reach it. 

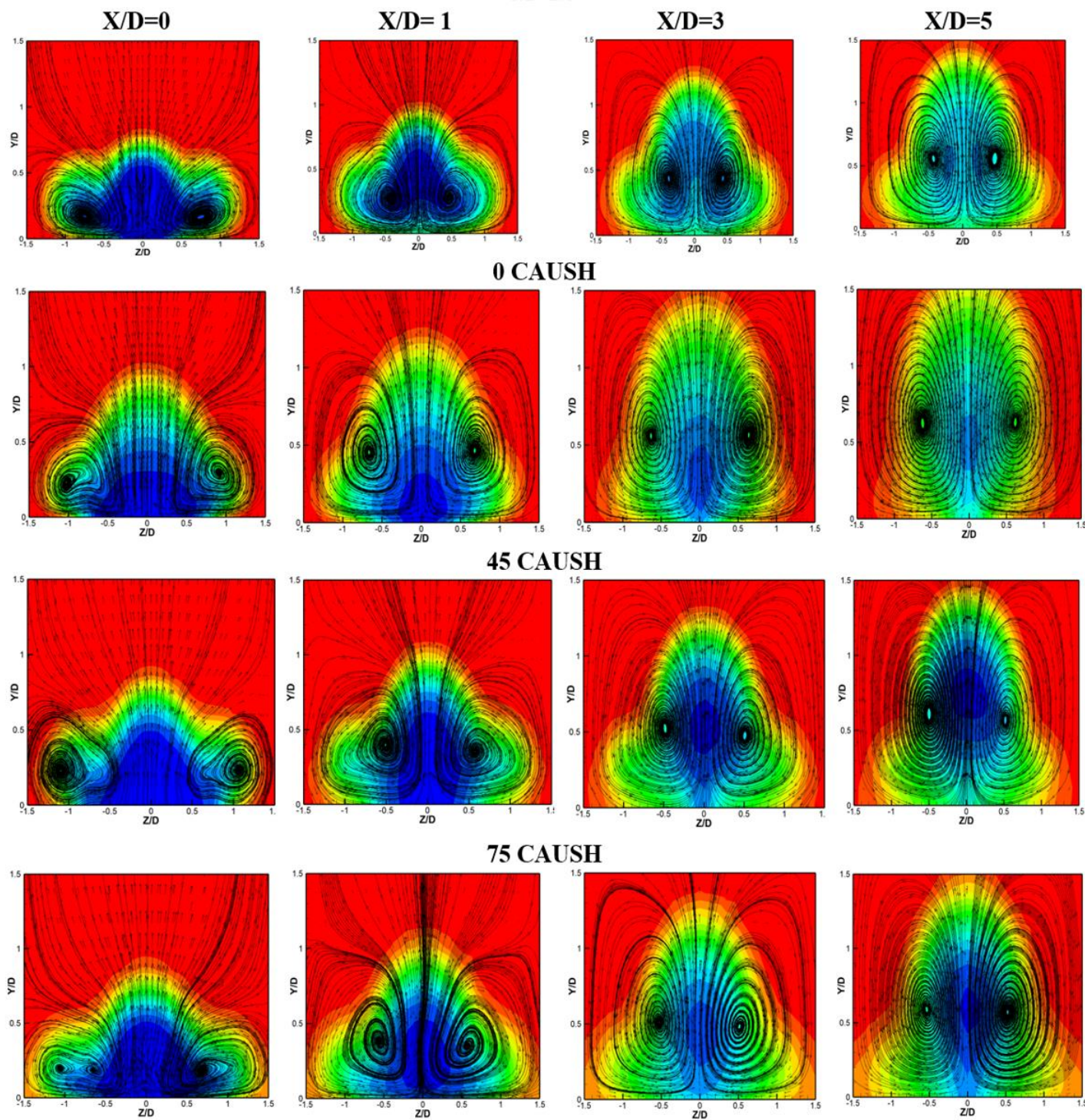

90 CAUSH

Temperature (K) $150160170180190200210220230240250260270280290300 \longleftrightarrow x$

Figure 6.17: Flow structure of the examined CAUSH at $M=1.5$ and $X / D=0,1,3$, and 5.

The most intense concentration of coolant that is entirely attached to the surface is presented in this case; $90^{\circ} \mathrm{CAUSH}$, and it delivers the best uniform lateral distribution for nearly the complete lateral domain at $(\mathrm{Z} / \mathrm{D}= \pm 1.5)$. Eventually, at $\mathrm{M}=1.0$, the $90^{\circ} \mathrm{CAUSH}$ yields the optimal centerline and lateral performance for the whole downstream region among the other examined blowing ratios and other CAUSH. On the other hand, Fig. 6.16 at $\mathrm{X} / \mathrm{D}=3$ and 5 shows that $0^{\circ}$ 
CAUSH has the worse coolant concentration and coverage close to the domain center because of the intense mixing, as shown by the temperature contours.

The flow structure results at the highest blowing ratio; $M=1.5$ is depicted in Figure 6.17. The plot shows that the coolant core is remaining notably adhesion to the plate surface for the entire holes vicinity region at $(\mathrm{X} / \mathrm{D}=0)$ and $(\mathrm{X} / \mathrm{D}=5)$ for $45^{\circ}, 75^{\circ}$, and $90^{\circ} \mathrm{CAUSH}$. Also, at $(X / D=0)$ and $(X / D=1)$, the generated ACRVP from various CAUSH is effectively defeating the CRVP of the main hole, whereas the coolant jet has high coolant momentum than that of the freestream. The $0^{\circ} \mathrm{CAUSH}$ at $(\mathrm{X} / \mathrm{D}=1)$, however, has exhibited minor entertainment and mixing occurs between the freestream and coolant jet underneath coolant core, which could be the reason of the fast deterioration in the centerline effectiveness in the hole vicinity region. As well, interestingly to see that the predicted flow structure results of the $75^{\circ} \mathrm{CAUSH}$ displayed in Figure 6.17 has revealed the broadest coolant distribution in the vertical direction in the hole vicinity region up to $(X / D<3)$, whereas the adherent coolant cover the domain of $( \pm 0.9,0.64)$ at $(X / D=0)$. The anti-counter rotating vortices pair structure produced for $75^{\circ} \mathrm{CAUSH}$ is accountable for its notable centerline effectiveness performance whereby their center is located apart from each other while it has a high coolant momentum. Therefore, ACRVP works very efficiently to eliminate the CRVP of the main hole, which enhanced the coolant enlargement vertically. On the other hand, $75^{\circ} \mathrm{CAUSH}$ at $(\mathrm{X} / \mathrm{D}=5)$ exhibits further mixing, which leads to coolant jet dissipation close to surface. The results of $90^{\circ} \mathrm{CAUSH}$ presented in Figure 6.17 show the best vertical and lateral coolant coverage amongst other CAUSH for the whole downstream region; however, slightly less than that attained at $M=1.0$. It is very important to point out that the spanwise position of the sister holes has a significant effect on the film cooling performance and flow structure as determined by Khajehhasani and Jubran [78]. They stated that placing the sister holes far from the center increased the performance and the lateral spreading of the coolant [78]. In the present investigation because of the hole design geometrical constraints each of the studied CAUSH has its precise spanwise location, (Z/D), that shown previously in Table 6.1 whereas the $90^{\circ} \mathrm{CAUSH}$ has the most far lateral location from the center of the primary hole, $Z / D= \pm 0.826$. This can be interpreted as one of the reasons that account for the high lateral performance of $90^{\circ} \mathrm{CAUSH}$ achieved for all examined blowing ratios among other CAUSH. Figure 6.17 shows the development of ACRVP at $(\mathrm{X} / \mathrm{D}=3)$ and $(\mathrm{X} / \mathrm{D}=5)$ for all CAUSH while its center is located far from the plate surface, which caused a further mixing of the coolant jet with the freestream on 
the vertical and lateral directions while the coolant attachment region to the surface is compacted and limited to the near area around center of the lateral domain.

\subsection{Conclusions}

In this chapter, the compound angle upstream sister hole (CAUSH) is proposed as a novel and simple hole scheme to improve the thermal performance of a cylindrical hole. Four compound angles; $\beta=0^{\circ}, 45^{\circ}, 75^{\circ}$, and $90^{\circ}$, are considered to determine the CAUSH film cooling effectiveness performance and the associated flow field at four blowing ratios $M=0.25-1.5$. It is revealed that all of the examined CAUSH demonstrated a substantial increase in the centerline and lateral film cooling performance for all examined blowing ratios for the whole downstream region. The $0^{\circ}, 45^{\circ}$, and $90^{\circ} \mathrm{CAUSH}$ provide the optimal cooling effectiveness at low blowing ratios $M=0.25$ and 0.5 . On the other hand, at high blowing ratios $M=1.0$ and 1.5 , cooling performance of all CAUSH is diverse dramatically and presented a distinct performance in the near and far-field downstream the hole exit; however, it can be said that the best performance is obtained by $0^{\circ}$ and $90^{\circ} \mathrm{CAUSH}$. The $90^{\circ} \mathrm{CAUSH}$ where sister holes are injected at $\beta=90^{\circ}$ at $\mathrm{M}=1.0$ offers the maximum overall film cooling effectiveness amongst all other CAUSH and blowing ratios. The ACRVP produced from the compound angle upstream sister holes play a significant role in controlling the flow field by keeping the coolant jet fully attached to the plate surface and restrain the coolant lift-off phenomena even at a very high blowing ratio. Consequently, distinguished lateral distribution and coolant coverage are attained. Overall, the compound angle upstream sister holes are noticeably enhanced the film cooling performance, and can be effectively applied to protect the high-pressure turbine blades and vans of aero-engines from the thermal failure and provide high film cooling coverage, specifically in the region that has a high surface curvature whereas most of the examined CAUSH the $0^{\circ}, 45^{\circ}$, and $90^{\circ}$ have shown an outstanding overall film cooling effectiveness for all examined blowing ratios. As well, CAUSH might be the best candidate to provide the necessary thermal protection for the turbine rotor double-wall airfoil in which its thin outer wall required a discrete film cooling holes that are either simple or compound injected. 


\section{Chapter Seven: Streamwise Location Effect of Compound Angle Sister Holes on Film Cooling Performance}

The outstanding cooling performance obtained from the proposed compound angle upstream sister hole (CAUSH) is the motivational reason for the study that is discussed in the present chapter. Whereas the compound angle sister holes were placed upstream the primary hole, it would be very interesting to assist the streamwise location effect of the compound angle sister hole (CASH) on the cooling performance. This parametric study will be conducted for three different streamwise locations relative to the center of the main hole specifically: upstream, midstream, and downstream compound angle sister holes at two blowing ratios for various CASH where $\beta=45^{\circ}, 75^{\circ}$, and $90^{\circ}$.

\subsection{Problem Statement}

It has been reported that sister holes film cooling performance is notably changed by the number of the sister holes being used, which are usually two and/or four sister holes placed at a specific location form the primary hole. Also, there is a number of parametric studies conducted in order to find the best location of the sister holes that gives the optimum performance [61] [64] [66]. Therefore, it is vital to find the optimum position of the sister holes either on spanwise or/ and streamwise that offers the highest performance. In this chapter, however, the investigation is limited to the effect of streamwise location on compound angle sister holes film cooling performance.

Not that, the 3D geometry investigated here is typically built based upon the compound angle upstream sister holes configuration studied in chapter six, which was relying on the single cylindrical hole case of Sinha et al. experimental study [89]. A brief overview of the 3D model is given herein, while the reader is encouraged to refer to chapter six for additional details. The main cylindrical hole with a diameter of $(D=12.7 \mathrm{~mm})$ and length to diameter ratio of $=1.75$ is injected at $(\alpha)=35^{\circ}$ to the flat plate. The origin point is located at the main hole (T.E) whereby the domain is extended 30D downstream it and 19D upstream the hole (L.E). The cooling hole modeled with $(\mathrm{P} / \mathrm{D})=3$; thus, the model has to be extended $3 \mathrm{D}$ in the lateral direction. The freestream channel height 10D is considered. The plenum is included with dimensions of (8D, $4 \mathrm{D}$, and $3 \mathrm{D})$ in (X, Y, and $\mathrm{Z}$ ), respectively. To add the compound angle sister holes to the main 
hole, two small sister holes, with a diameter of $(0.5 \mathrm{D})$ and same (L/D)ratio of the main hole, are added at several positions relative to the center of the main hole specifically: upstream, midstream, and downstream compound angle sister holes. For each position, the sister holes are injected symmetrically at several compound angles to the freestream direction at $\beta=45^{\circ}, 75^{\circ}$, and $90^{\circ}$ while the main hole is only streamwise injected. The sister holes position on $\mathrm{X}$-axis and Z-axis is considered a geometrical constraint for each case, as shown in Table 7.1, whereas this table lists the nine configurations with their dimensions of the cases that will be examined the compound angle sister holes (CASH) locations effect. Figure 7.1 shows a side view of the computational model, while Figure 7.2 presents a top view for the upstream position of the compound angle sister holes that focused only in the hole's vicinity region for the $45^{\circ} \mathrm{CASH}$, $75^{\circ} \mathrm{CASH}$, and $90^{\circ} \mathrm{CASH}$.

\subsection{Simulation parameters and Computational Overview}

The boundary conditions used in the simulation are chosen to be identical to the boundary conditions in the CFD study of Khajehhasani and Jubran for the single circular hole case [68][78]; which are premised on the experimental data of Sinha et al.[89]. Table 7.2 shows the mainstream and coolant flow parameters as well as the turbulence boundary conditions applied at the freestream and coolant inlets.

Table 7.1: The dimensions of the examined CASH configurations.

\begin{tabular}{|l|cccc||}
\hline The test cases of the examined CASH & X/D & Z/D & $(\alpha)$ & $(\beta)$ \\
\hline \hline$\left(45^{\circ}\right)$ Upstream & -0.693 & \pm 0.693 & $35^{\circ}$ & $45^{\circ}$ \\
$\left(45^{\circ}\right)$ Midstream & 0 & \pm 0.906 & $35^{\circ}$ & $45^{\circ}$ \\
$\left(45^{\circ}\right)$ Downstream & 0.693 & \pm 0.827 & $35^{\circ}$ & $45^{\circ}$ \\
$\left(75^{\circ}\right)$ Upstream & -0.693 & \pm 0.772 & $35^{\circ}$ & $75^{\circ}$ \\
$\left(75^{\circ}\right)$ Midstream & 0 & \pm 0.984 & $35^{\circ}$ & $75^{\circ}$ \\
$\left(75^{\circ}\right)$ Downstream & 0.693 & \pm 0.866 & $35^{\circ}$ & $75^{\circ}$ \\
$\left(90^{\circ}\right)$ Upstream & -0.693 & \pm 0.826 & $35^{\circ}$ & $90^{\circ}$ \\
$\left(90^{\circ}\right)$ Midstream & 0 & \pm 0.979 & $35^{\circ}$ & $90^{\circ}$ \\
$\left(90^{\circ}\right)$ Downstream & 0.693 & \pm 0.826 & $35^{\circ}$ & $90^{\circ}$ \\
\hline
\end{tabular}




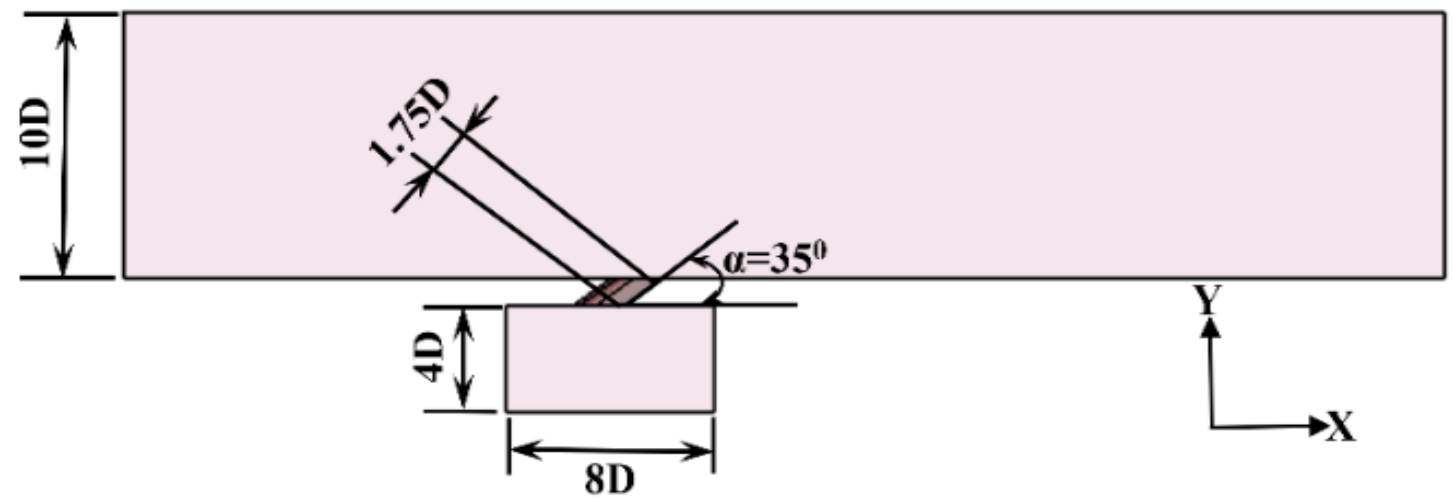

Figure 7.1: The side view of the computational model of the upstream $45^{\circ}$

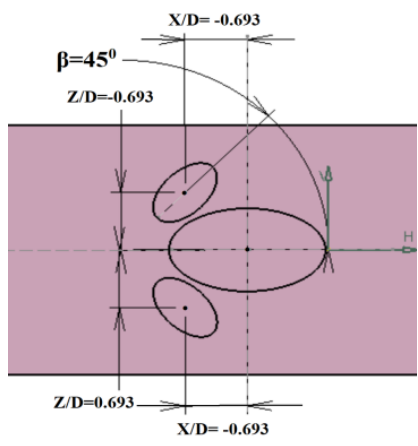

a) $45^{\circ} \mathrm{CAUSH}$
CASH. (Not to scale).

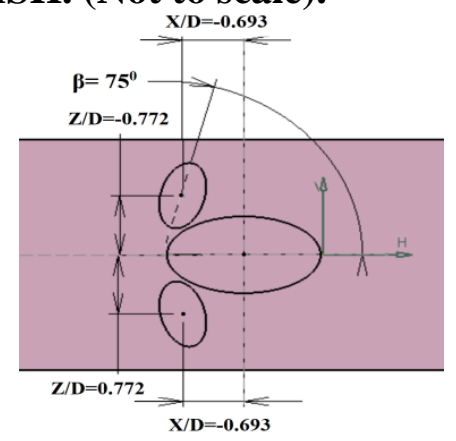

(d) $75^{\circ} \mathrm{CAUSH}$

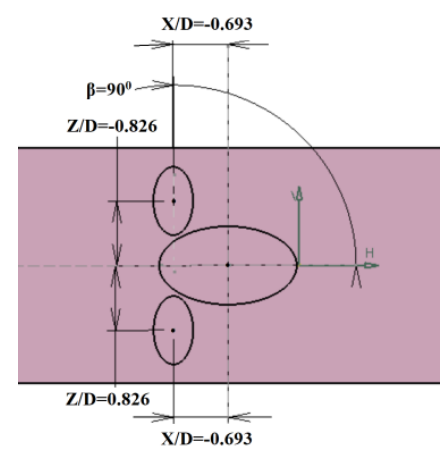

c) $90^{\circ} \mathrm{CAUSH}$

Figure 7.2: A top view magnifies the holes vicinity region for the upstream compound angle sister holes: a) $\beta=45^{\circ} \mathrm{CASH}$, b) $75^{\circ} \mathrm{CASH}$, and c) $90^{\circ} \mathrm{CASH}$, respectively. (Not to scale).

The cooling performance prediction of the compound angle sister holes at various locations and compound angles injection of sister holes are investigated at two blowing ratios; $\mathrm{M}=0.5$ is the low blowing ratio case, and $\mathrm{M}=1.5$ is the high blowing ratios case. Compound angle sister holes demanded 1.5 times more coolant than that of the discrete circular hole. Therefore, blowing ratio for the $\mathrm{CASH}$ can be achieved by keeping a constant freestream velocity $=20(\mathrm{~m} / \mathrm{sec})$ at the freestream inlet while varying the coolant velocity at plenum inlet. The coolant inlet velocity at the plenum inlet for the desired blowing ratios of the compound angle sister holes is presented in Table 7.3. Figure 7.3. shows the boundary conditions employed to the $3 \mathrm{D}$ model of the compound angle sister holes. 
Table 7.2: The mainstream and coolant flow parameters

\begin{tabular}{|l|c||}
\hline \multicolumn{1}{|c|}{ Property } & Value/units \\
\hline Free stream velocity & $20(\mathrm{~m} / \mathrm{sec})$. \\
Freestream temperature & $300 \mathrm{~K}$ \\
Freestream turbulence intensity & $0.2 \%$ \\
Freestream viscosity ratio & $50 \%$ \\
Coolant temperature & $150 \mathrm{~K}$ \\
Coolant turbulence intensity & $2.0 \%$ \\
Coolant viscosity ratio & $30 \%$ \\
Density ratio (D.R) & 2 \\
Operating pressure & $101.325(\mathrm{kPa})$ \\
\hline
\end{tabular}

Table 7.3: The coolant velocity at the plenum inlet.

\begin{tabular}{|c|c|}
\hline Blowing Ratio M & Compound sister holes Vc (m/sec) \\
\hline 0.5 & 0.245 \\
1.5 & 0.736 \\
\hline
\end{tabular}

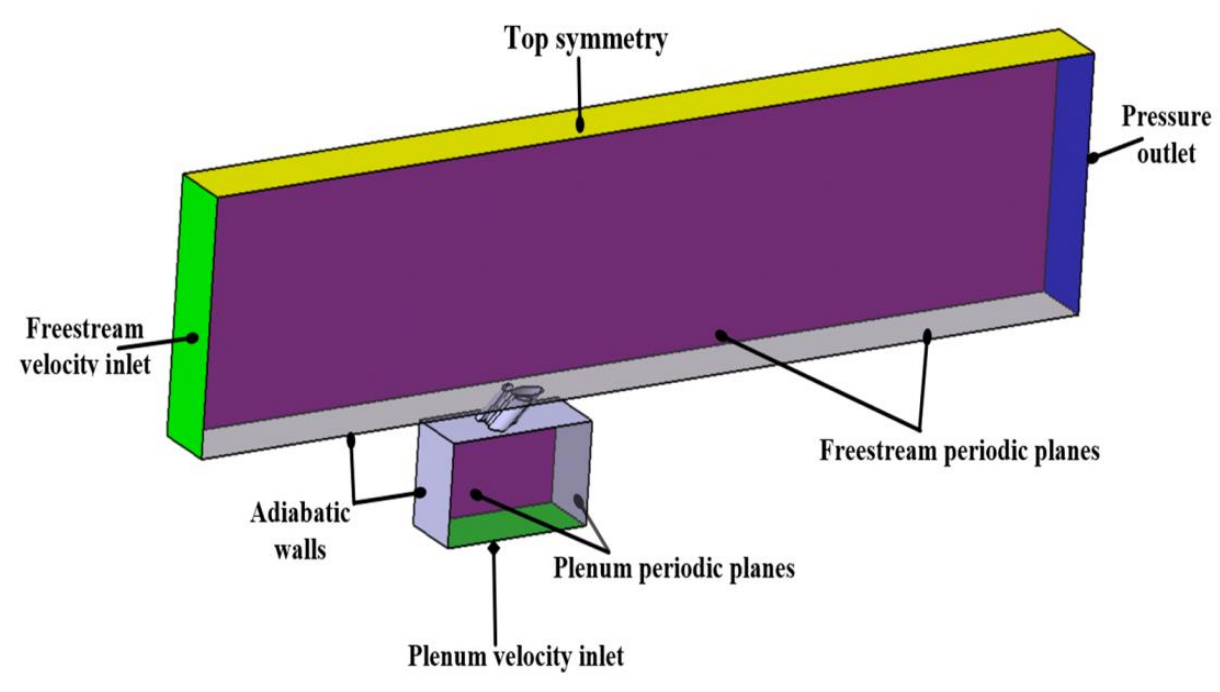

Figure 7.3: The 3D computational domain boundary conditions applied to the model for the upstream $45^{\circ} \mathrm{CASH}$ case . 
FLUENT-ANSYS 17.1 is used to run the simulation and solve the governing equations that define the thermal and flow field of the compound angle sister holes, which are the 3D RANS equations and the energy equation. Realizable k-epsilon is employed to model the turbulence of the flow field, and the near-wall modeling is completed using the enhanced wall treatment. The mesh of the computational domain is carefully generated on ANSYS-ICEM 17.1, utilizing the unstructured hexahedral mesh method. For an efficient resolution to the boundary layer and fast converge to the numerical solution, particular care has been paid to the generated mesh. This accomplished by a high grid quality in the zone near the cooling holes whereas O-grids are produced around the cooling holes and a very fine grid near the wall. Since the near-wall modeling is done via the enhance wall treatment, the mesh is formed very fine near the wall and the adjacent area of cooling holes area to attain the $(y+<1)$. However, far away from the wall, a coarser mesh is gradually generated to decrease the computational cost.

The numerical analysis is carried out in ANSYS-FLUENT 17.1, whereas it is processed as a steady-state, double-precision, and pressure base investigation. To mimic the experiment conditions, the working fluid is chosen as air that treated as incompressible and ideal flow. The second-order upwind scheme is used to be the discretization scheme of the momentum, energy, and turbulence model equations, while the SIMPLEC algorithm is used as a solution method for the velocity-pressure coupling. The solution of the numerical results is considered reaching convergence based on two parameters: (1) continuity equation and all velocity components normalized residuals are less than $\left(1 \times 10^{-4}\right)$ while energy equation residuals are less than $\left(1 \times 10^{6}\right)$, (2) the mass and energy imbalances for the entire computational domain is less than $0.001 \%$.

\subsection{Results and Discussion}

The predicted computational results of the compound angle sister holes for the investigated locations at various compound angles will be given here at two blowing ratios $\mathrm{M}=0.5$ and $\mathrm{M}=1.5$, and will be presented in terms of centerline effectiveness, laterally averaged effectiveness, local film cooling distribution at the plate surface, and the flow structure results to assess the compound angle sister holes locations influence on film cooling performance. 


\subsubsection{The Film Cooling Effectiveness Results of the Compound Angle Sister Holes at Three Streamwise Locations}

The centerline effectiveness results of the compound angle sister holes at low blowing ratio; $\mathrm{M}=0.5$, are shown in Figure 7.4 whereas a comparison of the cooling performance at three streamwise positions: upstream, midstream, and downstream is completed for each compound angle sister holes. The plot clearly shows that for all examined compound angles sister holes; $45^{\circ}$, $75^{\circ}$, and $90^{\circ} \mathrm{CASH}$, placing the sister holes downstream has significantly improved the cooling performance for the entire downstream region. On the other hand, the other two locations, the upstream and the midstream, have shown almost the same performance in the holes adjacent region up to (X/D>5), and then the midstream location shows slightly better performance than that of the upstream for the $45^{\circ}$ and $90^{\circ} \mathrm{CASH}$. Interestingly, the midstream location of $75^{\circ}$ CASH has a bit preferable performance than the other two locations at $(X / D \geq 20)$. Note that at a low blowing ratio, the coolant jet stays attached to the plate surface. So, when sister holes are placed downstream, less interaction with the freestream occurs that leads to better performance. The downstream location of the compound angle sister holes has increased the centerline effectiveness by about $14 \%, 31 \%$, and $17 \%$ for the $45^{\circ}, 75^{\circ}$, and $90^{\circ} \mathrm{CASH}$, respectively, compared to the performance attained by the upstream location at a blowing ratio of $\mathrm{M}=0.5$.

Figure 7.5 presents the centerline performance of the compound angle sister holes at the three streamwise locations at high blowing ratio $\mathrm{M}=1.5$. Unlike the steady performance at low blowing ratio, a large variation in the centerline effectiveness is noticed in near hole region and far downstream. The downstream position of the compound sister holes is still offering the best cooling performance for all examined compound angles; mainly in the hole adjacent region at (X/D < 7) for $75^{\circ}$ and $90^{\circ} \mathrm{CASH}$. The $45^{\circ} \mathrm{CASH}$ centerline effectiveness, shown in fig. $7.5 \mathrm{a}$ ), demonstrates that downstream location offers about $30 \%$ an increase in the performance up to the downstream region $(\mathrm{X} / \mathrm{D}<20)$ in contract to the performance achieved by the upstream location. The upstream and the midstream locations have not shown a comparable performance like that attained at a low blowing ratio. Instead, the upstream and the downstream cooling performance is more like, specifically in the vicinity region of the cooling holes at $(\mathrm{X} / \mathrm{D}<5)$. In the far downstream region of the hole exit, the three locations have demonstrated a major 


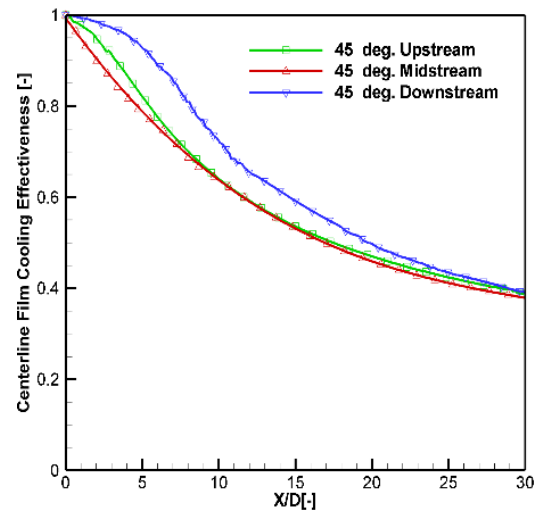

a) $45^{\circ} \mathrm{CASH}$

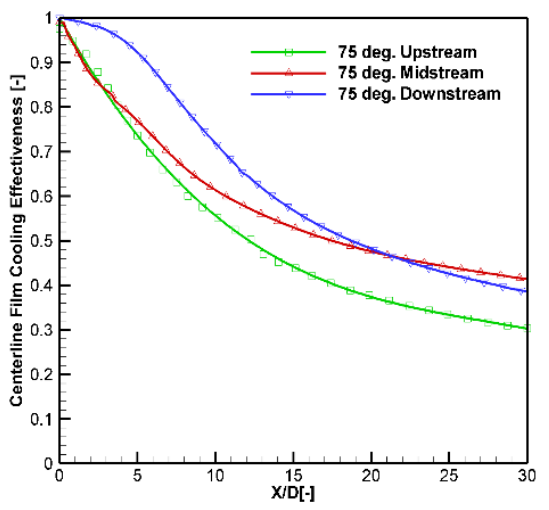

b) $75^{\circ} \mathrm{CASH}$

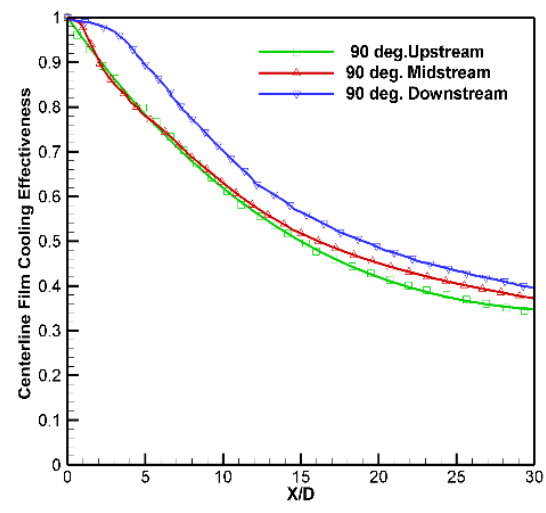

c) $90^{\circ} \mathrm{CASH}$

Figure 7.4: a) -c) The centerline film cooling effectiveness for the $45^{\circ}, 7^{\circ}$, and $90^{\circ}$ $\mathrm{CASH}$, respectively, at $\mathrm{M}=\mathbf{0 . 5}$.

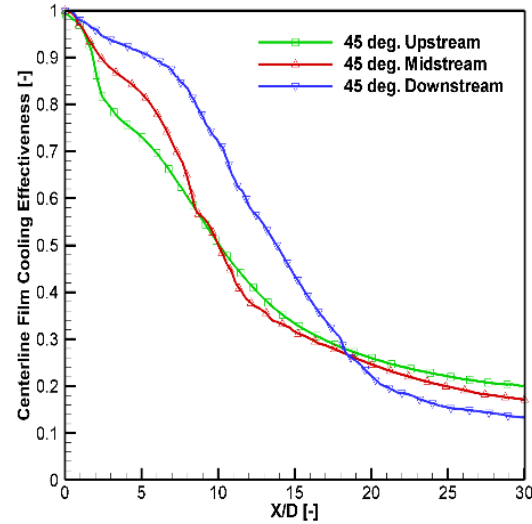

a) $45^{\circ} \mathrm{CASH}$

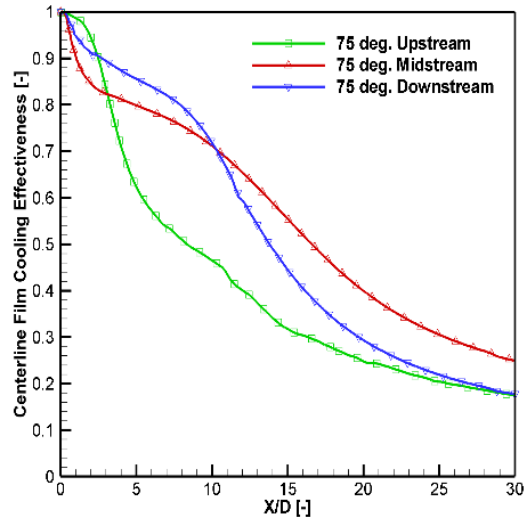

b) $75^{\circ} \mathrm{CASH}$

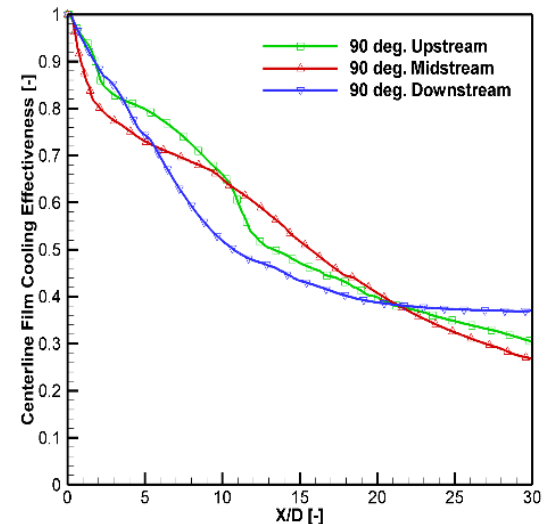

c) $90^{\circ} \mathrm{CASH}$

Figure 7.5: a) -c) The centerline film cooling effectiveness for the $45^{\circ}, 7^{\circ}$, and $90^{\circ}$ CASH, respectively, at $\mathrm{M}=1.5$.

deterioration in the performance compared to that attained at low blowing ratio. Also, interestingly to observe that the three locations give an identical performance at $(X / D \approx 20)$ for the $45^{\circ}$ and $90^{\circ} \mathrm{CASH}$, as shown in fig. 7.5 a) and c), while after that a variation on their performance has appeared. Whereas the upstream position of $45^{\circ} \mathrm{CASH}$ exhibits a slight increase and optimum performance compared to the other locations, as shown in Fig. 7.5 a), while the downstream location of $90^{\circ} \mathrm{CASH}$ shows the best performance among other locations, as depicted in Fig. $7.5 \mathrm{c}$ ). The $75^{\circ} \mathrm{CASH}$ upstream location shows the highest performance at (X/D $<5$ ), and then its cooling performance is considerably reduced to give the lowest performance compared to the other two locations further downstream. On the other hand, the $75^{\circ} \mathrm{CASH}$ 


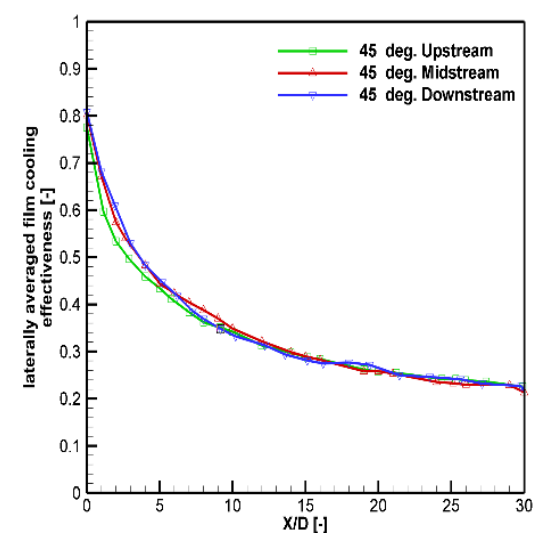

a) $45^{\circ} \mathrm{CASH}$

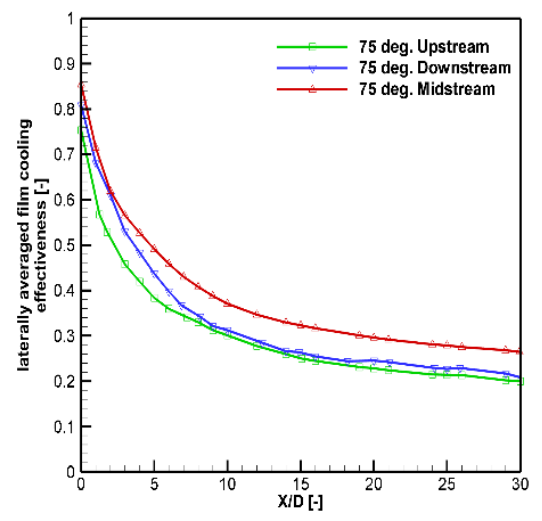

b) $75^{\circ} \mathrm{CASH}$

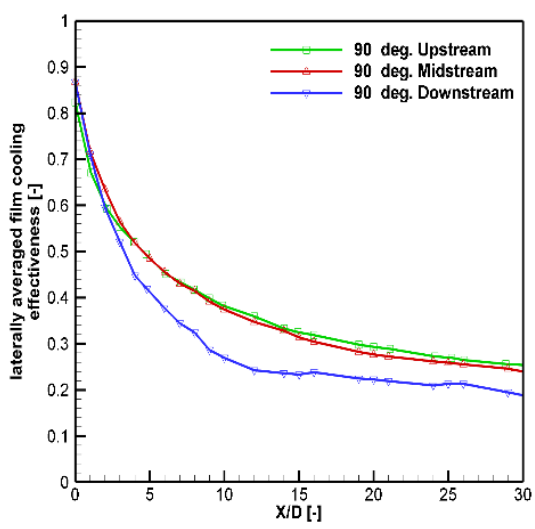

c) $90^{\circ} \mathrm{CASH}$

Figure 7.6: a) -c) The Laterally Averaged Film Cooling Effectiveness for the $45^{\circ}$, $75^{\circ}$, and $90^{\circ} \mathrm{CASH}$, respectively, at $\mathrm{M}=0.5$.

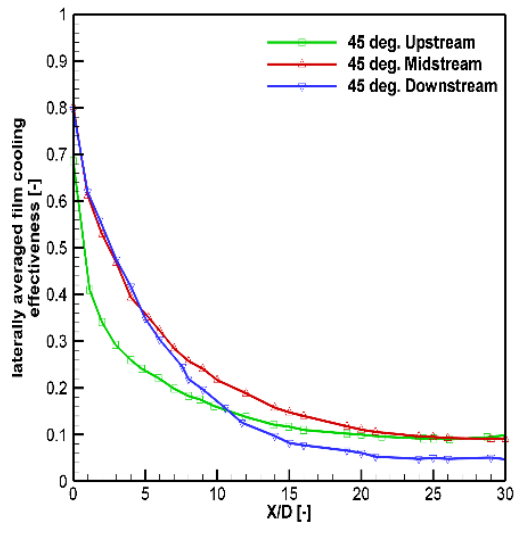

a) $45^{\circ} \mathrm{CASH}$

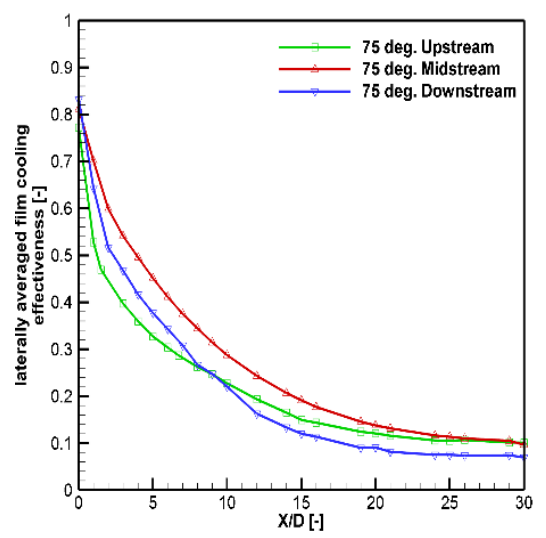

b) $75^{\circ} \mathrm{CASH}$

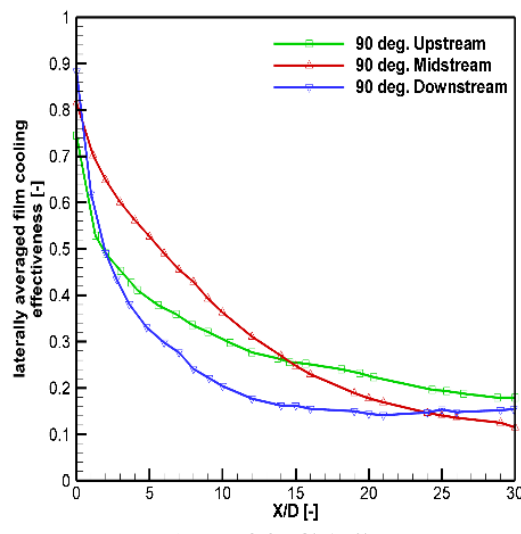

c) $90^{\circ} \mathrm{CASH}$

Figure 7.7: a) -c) The Laterally Averaged Film Cooling Effectiveness for the $45^{\circ}$, $75^{\circ}$, and $90^{\circ} \mathrm{CASH}$, respectively, at $\mathrm{M}=1.5$.

midstream position exhibits the best performance at $(5<\mathrm{X} / \mathrm{D}<15)$ while the downstream location of the $75^{\circ} \mathrm{CASH}$ provides a notable increase in the centerline effectiveness further downstream at $(\mathrm{X} / \mathrm{D}>15)$, as depicted in Fig. $7.5 \mathrm{~b})$.

The laterally averaged film cooling effectiveness results of the three streamwise locations of the compound angle sister holes at low blowing ratio; $M=0.5$, is depicted in Figure 7.6. In general, at low blowing ratio, there is an insignificant variation in the performance among the examined holes positions; precisely in the hole vicinity area, but further downstream, a small change in the performance is noticed for the $75^{\circ}$ and $90^{\circ} \mathrm{CASH}$. Figure 7.6 a) illustrates that for $45^{\circ} \mathrm{CASH}$, the performance is indistinguishable from varying the sister holes locations. Fig. 7.6 b) shows that the midstream and downstream locations of $75^{\circ} \mathrm{CASH}$ present a higher 
performance than the upstream location while further downstream at $(X / D>10)$ the downstream location cooling performance decreases to approach the upstream location cooling performance. The midstream location of $75^{\circ} \mathrm{CASH}$ has increased the lateral effectiveness by approximately $30 \%$ in contract to the performance reached by the upstream location. Fig. $7.6 \mathrm{c}$ ) exhibit that optimum cooling performance of the $90^{\circ} \mathrm{CASH}$ immediately at the hole exit is attained by the downstream and midstream location while further downstream the upstream and midstream locations offer exactly the same performance.

The predicted lateral performance results of the tested streamwise locations of the compound angle sister holes at high blowing ratio; $M=1.5$, is presented in Figure 7.7. Overall. the midstream position of the sister holes yields the best performance for the whole downstream area while the downstream position offers the worse performance on the far downstream. Also, the $90^{\circ} \mathrm{CASH}$ midstream is giving the optimum performance amongst all other cases at this high blowing ratio. At blowing ratio of $\mathrm{M}=1.5$, the midstream location of the compound angle sister holes has offered a notable improvement in the lateral effectiveness that is around $50 \%, 40 \%$, and $28 \%$ for the $45^{\circ}, 75^{\circ}$, and $90^{\circ} \mathrm{CASH}$, respectively compared to the lateral effectiveness accomplished by the upstream location.

To determine which of nine examined cases of the compound angle sister holes positions are offering the best cooling performance, a comparison of the centerline and lateral cooling performance for all cases at two blowing ratios is performed. Figure 7.8 a) and b) show the results of the centerline and laterally averaged effectiveness for all examined locations of various CASH at $\mathrm{M}=0.5$, while Fig. 7.9 a) and b) exhibit the centerline and laterally averaged effectiveness for all examined locations of various $\mathrm{CASH}$ at $\mathrm{M}=1.5$. It can be seen from Fig. 7.8 a) that placing the sister holes downstream enhances the centerline effectiveness for the entire downstream for all examined compound angles, whereas the maximum performance is attained by $45^{\circ} \mathrm{CASH}$ downstream. On the other hand, the upstream location presents the lowest centerline performance, whereby the $75^{\circ} \mathrm{CASH}$ upstream has minimal performance. The lateral performance at $\mathrm{M}=0.5$ shown in Fig. $7.8 \mathrm{~b}$ ) shows that all of the locations of the sister holes nearly show the same performance immediately downstream the hole exit, while the downstream and midstream locations provide the best performance within the hole adjacent at $(\mathrm{X} / \mathrm{D}<5)$. Further downstream; however, the midstream and upstream locations show better performance. The optimal and almost identical performance is obtained by both the $90^{\circ} \mathrm{CASH}$ 


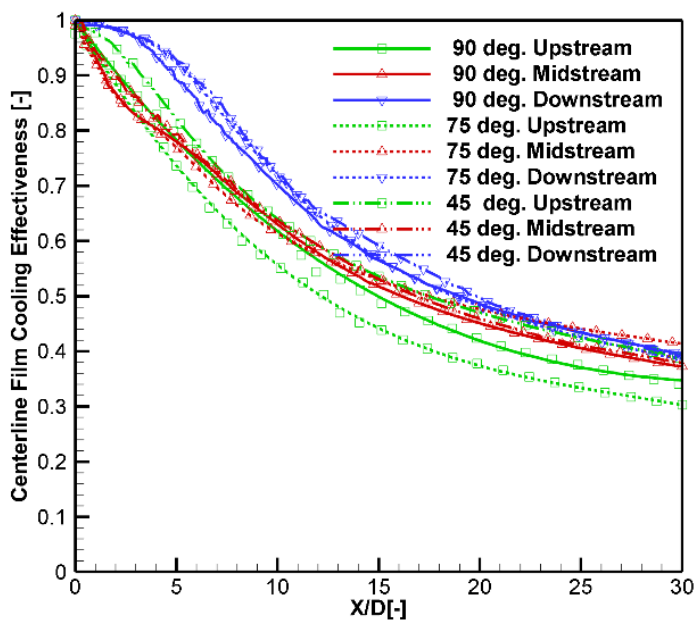

a) Centerline effectiveness

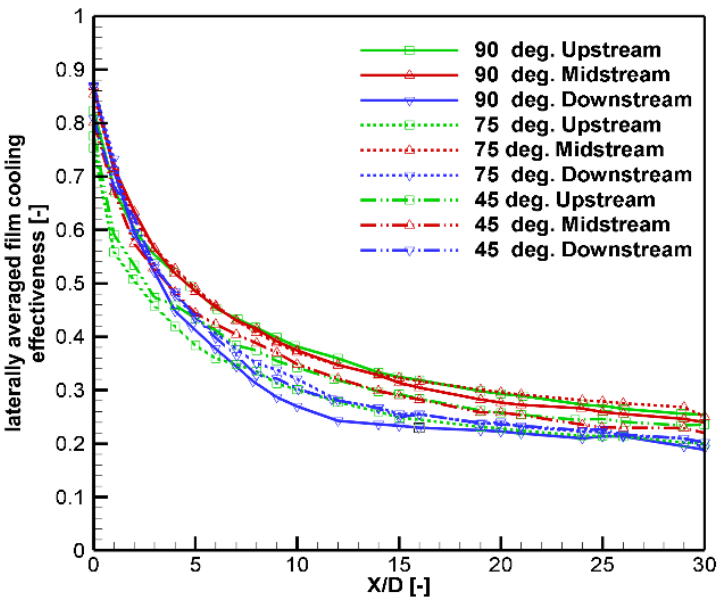

b) Laterally Averaged Effectiveness

Figure 7.8: The comparison of a) centerline effectiveness and b) laterally averaged effectiveness for all examined locations and CASH at M=0.5.

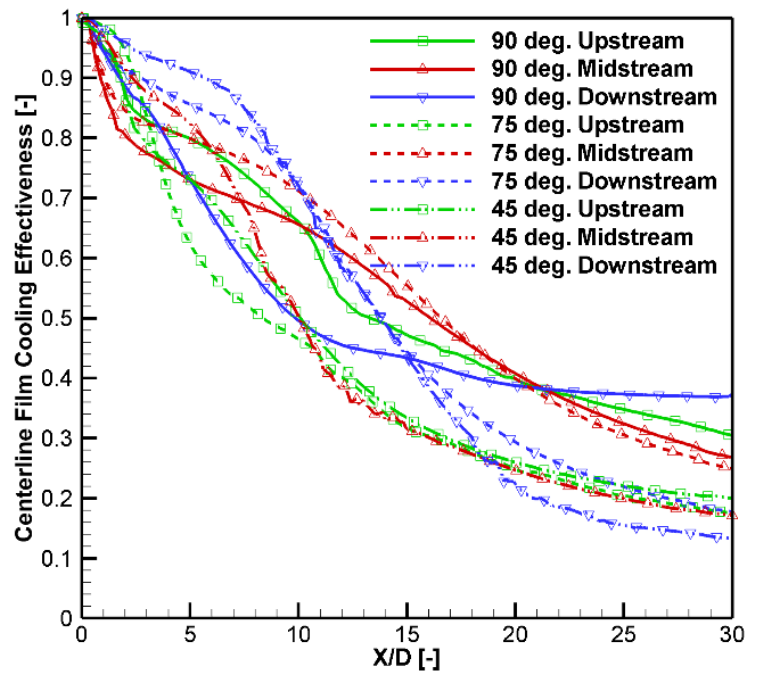

a) Centerline effectiveness

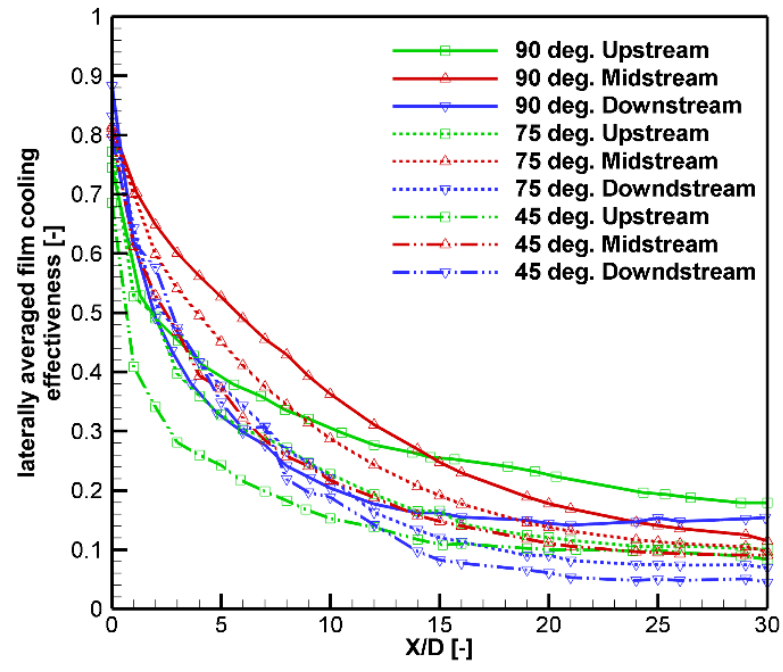

b)Laterally Averaged Effectiveness

Figure 7.9: The comparison of a) centerline effectiveness and b) laterally averaged effectiveness for all examined locations and CASH at $\mathrm{M}=1.5$.

upstream and midstream as well as the $75^{\circ} \mathrm{CASH}$ midstream for the entire downstream area. But, the $90^{\circ} \mathrm{CASH}$ downstream offers slightly higher performance at $(\mathrm{X} / \mathrm{D}<3)$. The cooling performance at high blowing ratio $\mathrm{M}=1.5$ is more distinct in contrast to the performance attained at $\mathrm{M}=0.5$. in terms of the centerline effectiveness. Fig. 7.9 a) shows that the downstream location offers the best performance up to $(\mathrm{X} / \mathrm{D} \approx 10)$, while after that the midstream location of CASH exhibits better centerline effectiveness within the range of $(10<\mathrm{X} / \mathrm{D}>20)$. There are, however, a wide region of overlapping among the results at $(3<\mathrm{X} / \mathrm{D}>25)$ and no general trend can be observed. In other words, not all the downstream location of the compound angle sister holes 
enhances the performance in the hole vicinity region whereas the case of the $90^{\circ} \mathrm{CASH}$ downstream has the lowest effectiveness compared to the other two compound angles at the same position. A similar trend is existing farther downstream, whereas the $45^{\circ} \mathrm{CASH}$ midstream is not working effectively as the other examined angles at this location. It has been found herein in chapter six that the upstream compound angle sister holes have increased the overall cooling effectiveness in contrast to the discrete round hole by a factor of $1.97,1.91$, and 2.26 for the $45^{\circ}$, $75^{\circ}$, and $90^{\circ}$ (CAUSH) respectively [101]. Therefore, based on the results shown in Fig. 7.8 and Fig. 7.9, it can be fairly said that the other locations of the compound angle sister holes increase the overall cooling performance at least twice than that of a single round hole.

The results of the flow field of the upstream compound angle sister holes studied in chapter six had demonstrated that the significant increase achieved on the performance at high blowing ratios has resulted from restraining the coolant jet from lifting- off from the plate surface with less mixing. Thus, the analysis of the local film effectiveness distribution at the plate surface for all examined locations of the compound angle sister holes will be presented next at two blowing ratios $\mathrm{M}=0.5$ and 1.5 to understand how the location of the compound angle sister holes affect the coolant jet attachment, coolant coverage, and the cooling performance. Figure 7.10 shows a 2D contours plot on $\mathrm{X}-\mathrm{Z}$ plane for the predicted results of local film cooling effectiveness at the plate surface at low blowing ratio $M=0.5$, while Fig. 7.11 exhibits the results at high blowing ratio $M=1.5$. Figure 7.10 and Figure 7.11 generally show a symmetrical distribution of the coolant at the plat surface around $\mathrm{X}$-axis as well as a superior uniform coolant coverage and local cooling performance is achieved up to $(X / D<10)$ for the streamwise and the spanwise directions. The blowing ratio $\mathrm{M}=0.5$ yields an outstanding cooling performance of all $\mathrm{CASH}$ locations for the whole downstream area; on the other hand, blowing ratio $\mathrm{M}=1.5$ excellent cooling performance is limited to $(\mathrm{X} / \mathrm{D} \approx 17)$. Moreover, all examined locations of the compound angle sister holes hold the coolant jet adhesion to the plate surface at low and high blowing ratios whilst a considerable coolant coverage is revealed. Figure 7.10 shows that the midstream location of the compound angle sister holes shows the best lateral coolant coverage and uniformity, whereas the coolant jet covers the entire lateral domain $( \pm 1.5 \mathrm{D})$. Also, the optimum centerline coolant coverage is achieved by placing the compound angle sister holes downstream, whereas a notable coolant concentration is appeared up to $(X / D \approx 15)$. The least coolant coverage for the whole downstream area is shown by $75^{\circ} \mathrm{CASH}$ upstream case, whereas an intense mixing seems 

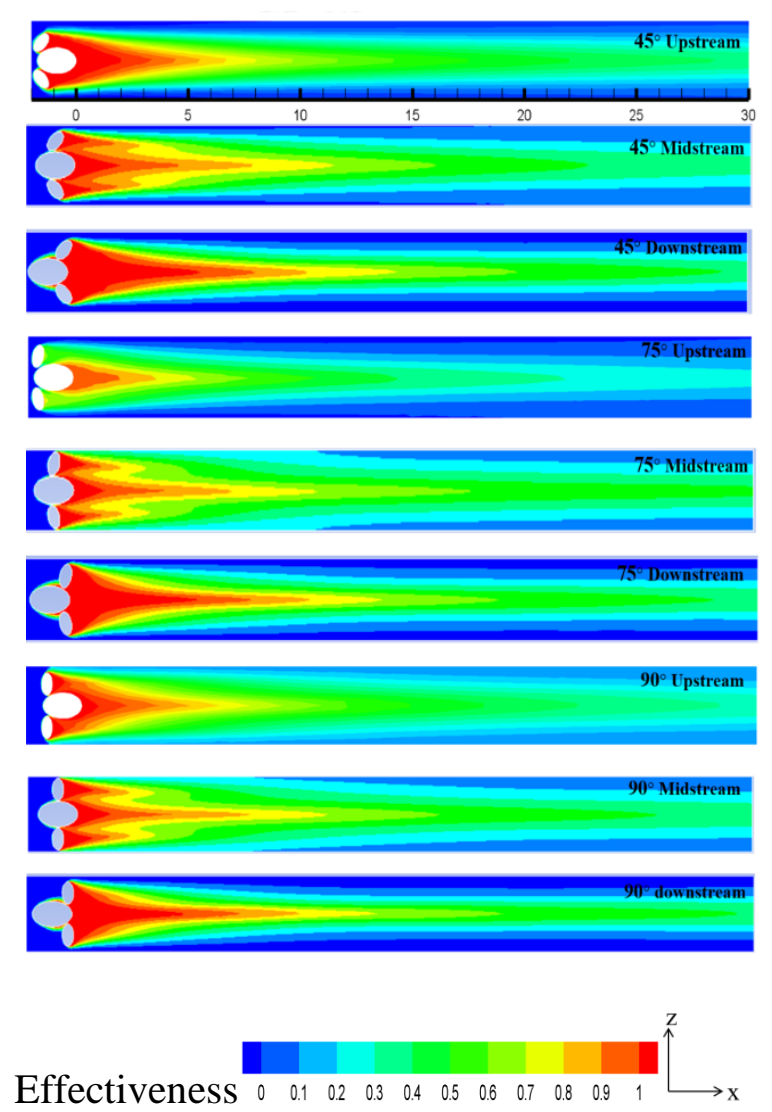

Figure 7.10: The local film cooling effectiveness for various CASH locations at $\mathrm{M}=\mathbf{0 . 5}$.
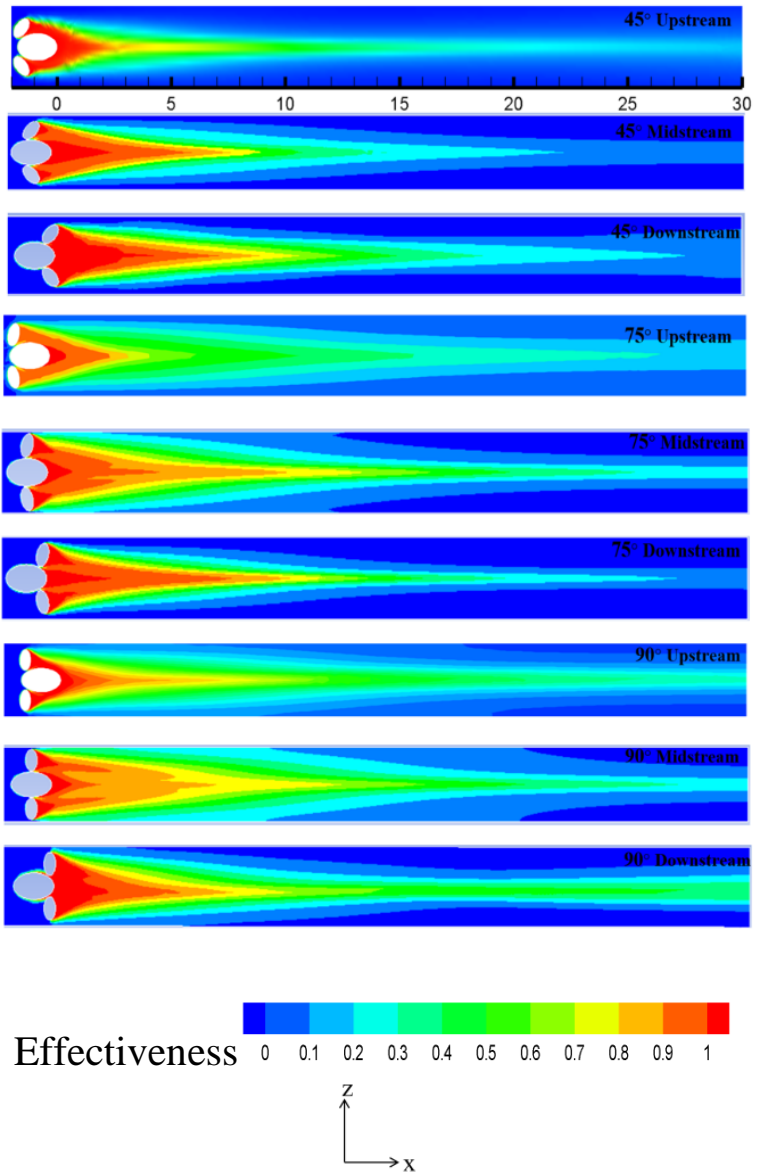

Figure 7.11: The local film cooling effectiveness for various CASH locations at $\mathbf{M}=\mathbf{1 . 5}$.

mainly occurred in the sister holes region.

Figure 7.11 shows that local effectiveness distribution is significantly high at this high blowing ratio; $M=1.5$, as well as the coolant jet stays adherent to the plate surface up to $(X / D \approx$ 10). However, a decline in the local cooling effectiveness and coolant coverage are observed further downstream at $(\mathrm{X} / \mathrm{D}>10)$, which is attributed to the intensive mixing and penetration of the coolant jet into the freestream whereas the coolant jet has a higher momentum than the freestream. The $45^{\circ}$ and $75^{\circ} \mathrm{CASH}$ upstream both show low performance in the hole vicinity region compared to other examined $\mathrm{CASH}$ angles and locations. The flow structure presence in anti-counter rotating vortices pair has contributed to the notable cooling performance attained from the various locations of the compound angle sister holes, whereas the results will be presented in the next section. 


\subsubsection{The Flow Structure Results}

Flow structure and it's associated generated vortices of the compound angle sister holes have a predominant effect on the predicted film cooling performance discussed previously. Therefore, to have a better understanding of the CASH cooling performance, it is essential to visualize this flow structure through the use of the temperature contours, velocity vectors, and streamlines. The visualization of the results of the CASH flow field and vorticial structures will be given at three locations; plans perpendicular to the plate surface; $\mathrm{X} / \mathrm{D}=0,1$, and 5 for two blowing ratios $\mathrm{M}=0.5$ and 1.5. Velocity vectors and streamlines are presenting the produced vortices strength, size, and direction of rotation, while temperature contours are indicating the mixing rate and the interaction of the coolant jet with the freestream. The flow structure results of the upstream location of the compound angle sister holes will not be displayed here since they are presented in detail in chapter six. The flow structure results at low blowing ratio $\mathrm{M}=0.5$ and high blowing ratio $\mathrm{M}=1.5$ are shown in Figures 7.12 and Figure 7.13, respectively.

Flow structure results of the midstream location of various compound angle sister holes at $\mathrm{M}=0.5$, shown in Figures $7.12 \mathrm{a}$ ), display that the coolant jet core is attached to the plate surface for all examined $\mathrm{CASH}$ for entire hole vicinity region ( $\mathrm{X} / \mathrm{D}=0$ to 5). As a result of low coolant jet momentum, there is less penetration of the coolant jet into the freestream, but more mixing is occurred in the lateral and vertical directions by moving further downstream as shown specifically at $(X / D=5)$. Interestingly, at $(X / D=0)$ the plot shows the generation of the two pairs of anticounter rotating vortices where their centers are located very close to the plate surface for all $\mathrm{CASH} ; 45^{\circ}, 75^{\circ}$, and $90^{\circ}$. Thus, they effectively work to defeat and lessen the strength of the CRVP of the main hole. Also, for all CASH, the coolant core concentration is symmetrically distributed around Z-axis, and it seems that the coolant jet emerged from sister holes well support the coolant jet emerged out of the main hole to covers a wide lateral domain $(\approx \pm 1.25)$. At $(\mathrm{X} / \mathrm{D}=1)$, the coolant core distribution starts to get compacted on the lateral domain. Moreover, the ACRVP and CRVP are developed while the two pairs of ACRVP combined on one pair with a larger size and located very close to lateral domain boundary which effectively beat the CRVP of the main hole and maintains the coolant jet attached to the 
$\mathbf{X} / \mathbf{D}=\mathbf{0}$
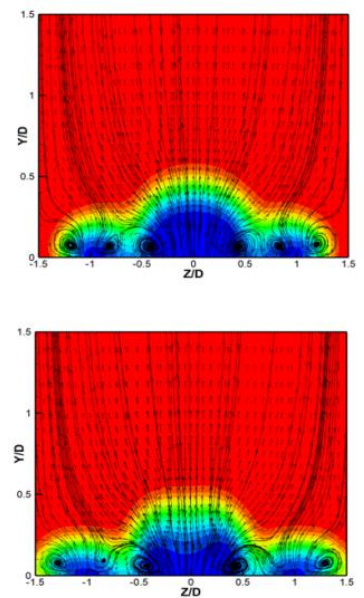

zio

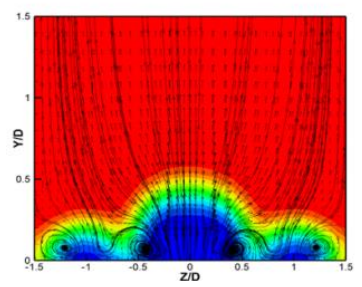

$\mathbf{X} / \mathbf{D}=\mathbf{0}$
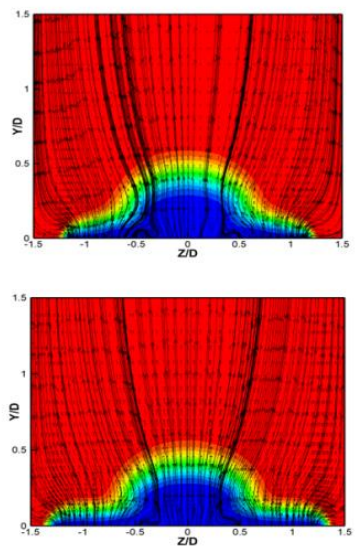

zio

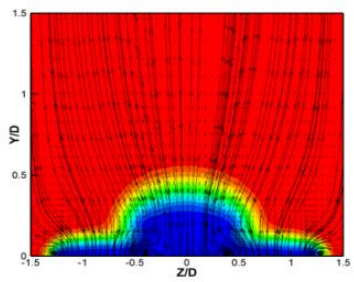

b) Downstream location

$90^{\circ}$ CASH

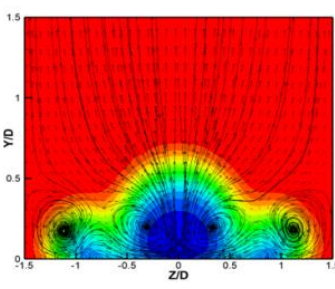

$45^{\circ} \mathrm{CASH}$

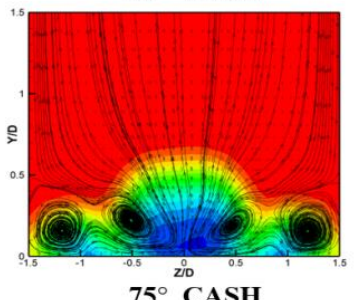

$75^{\circ}$ CASH
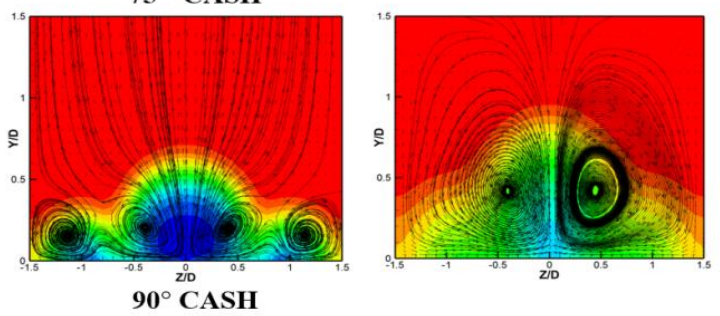

a) Midstream location

$\mathrm{X} / \mathbf{D}=\mathbf{1}$

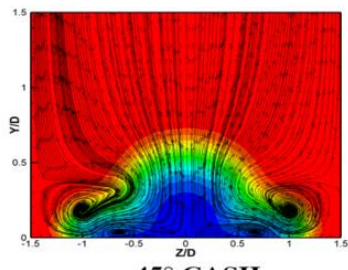

$45^{\circ} \mathrm{CASH}$
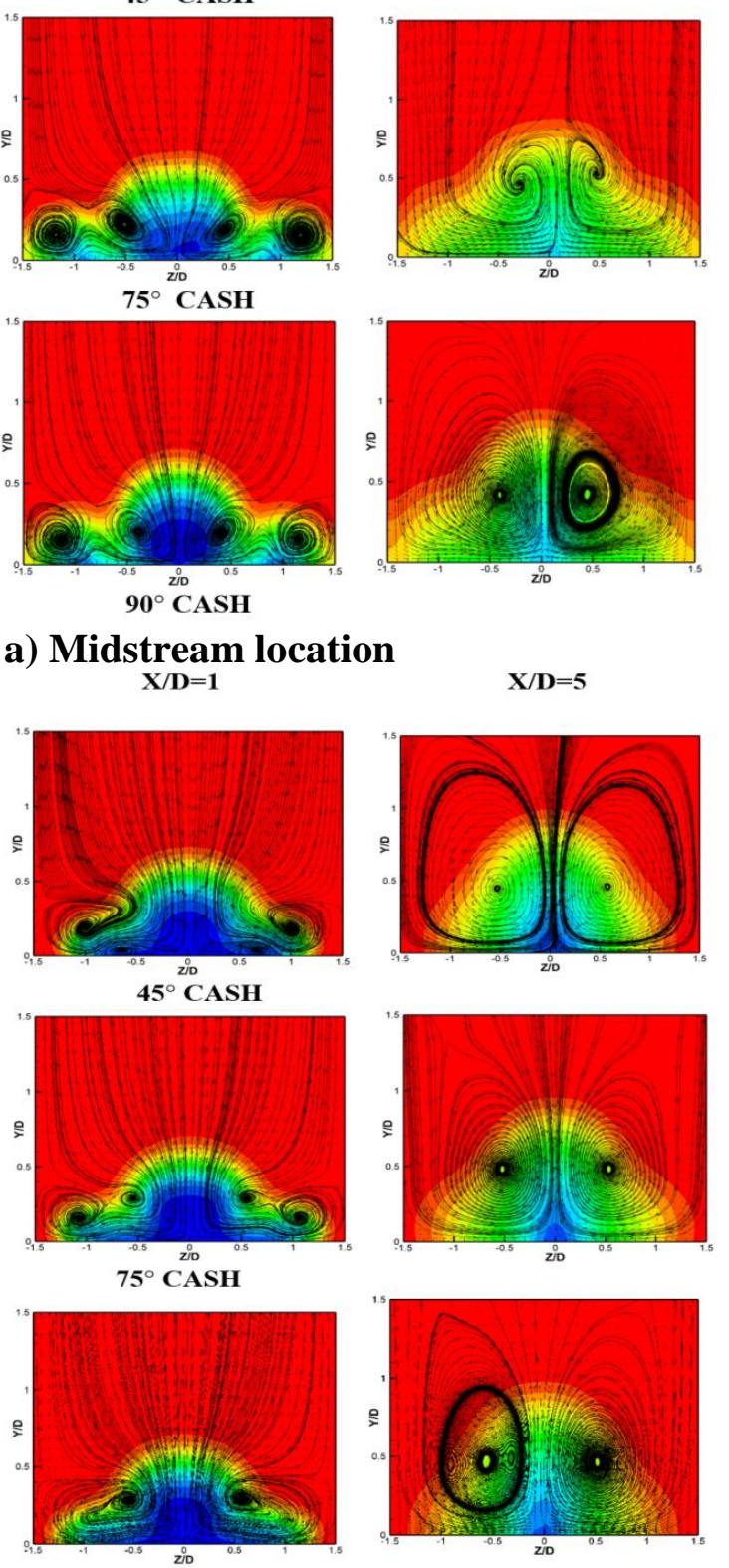

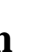

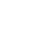

Temprature (K) $150160170180190200210220230240250260270280290300 \stackrel{\text { L }}{\longrightarrow}$

Figure 7.12: The flow structure results of $\mathrm{CASH}$ at $\mathrm{X} / \mathrm{D}=0,1$, and 5 a) midstream and b) downstream at $\mathrm{M}=0.5$. 
plate surface. At $(\mathrm{X} / \mathrm{D}=5)$, a less coolant core attachment region is observed with more mixing in the vertical and lateral directions. Furthermore, the ACRVP is developed further and become predominant vortices structure while the CRVP is diminished. But because of the occurrence of the intense mixing in this location for all examined $\mathrm{CASH}$, a reduction in the thermal performance is observed further downstream. The downstream locations flow structure results of the compound angle sister holes at $\mathrm{M}=0.5$, depicted in Figures $7.12 \mathrm{~b}$ ), illustrate that, in general, and for all examined $\mathrm{CASH}$, The coolant core remains significantly attached to the plate surface not only at $(X / D=0)$ but also at $(X / D=5)$. At $(X / D=0)$, it shows a wider uniform coolant distribution that covers almost all of the lateral domain $(\approx \pm 1.45)$, and the freestream has not entertained with the coolant jet close to the plate surface. In terms of the vortices structure, there is one pair of the ACRVP and CRVP located close to each other while they are not strong, which is different from the midstream location cases. At $(\mathrm{X} / \mathrm{D}=1)$ the coolant still covers a wider domain, and it is a bit compacted laterally and expanded vertically than that at $(X / D=0)$. The ACRVP is stronger than the CRVP and has large downwash vorticity that holds the coolant attached to the surface. At $(X / D=5)$, in terms of the vortices structure, the same result is found in contrast to that for the midstream location, whereas a further development of the ACRVP is revealed with a nearly diminished CRVP. However, the coolant core distribution and the cover region close to the center of the spanwise domain are better than that for the midstream location of the compound angle sister holes. For this reason, the downstream location of the compound angle sister holes showed better centerline cooling performance compared to the other two studied locations at $\mathrm{M}=0.5$.

The predicted flow structure results of $\mathrm{CASH}$ at high blowing ratio $\mathrm{M}=1.5$ are illustrated in Figure 7.13 a) and b) for the midstream and downstream locations, respectively. It is very interesting to observe from Figure 7.13 a) and b) that at this high blowing ratio the coolant core remains considerably attached to the plate surface from $(X / D=0)$ to $(X / D=5)$. Moreover, there is a significant coolant core distribution in the vertical and lateral directions, which demonstrates the great benefit of using either location of $\mathrm{CASH}$; midstream and downstream, to sustain the coolant adhesion and provide uniform coolant coverage to the plate surface. The plot obviously shows that the coolant coverage and the uniform distribution attained in the hole vicinity region; $(\mathrm{X} / \mathrm{D}=0$ to 5 ) at those two locations, is higher than that obtained at $\mathrm{M}=0.5$. For both locations, since the coolant jet has high momentum compared to that of the freestream, at $(X / D=0)$ the 
$\mathbf{X} / \mathbf{D}=\mathbf{0}$

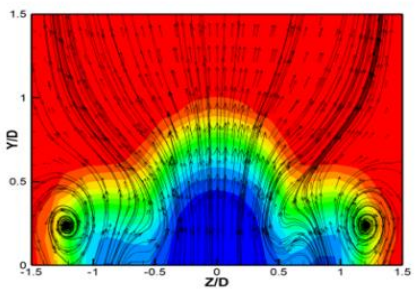

zio
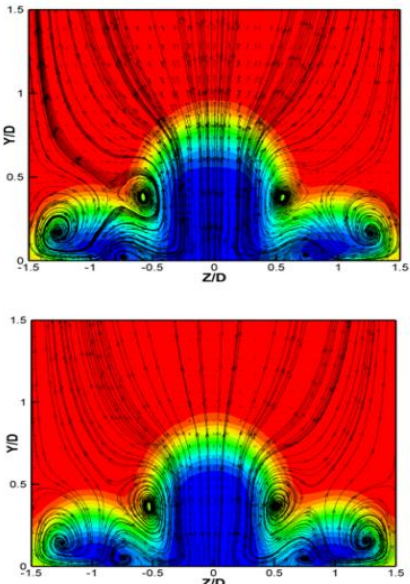

$\mathbf{X} / \mathbf{D}=\mathbf{0}$
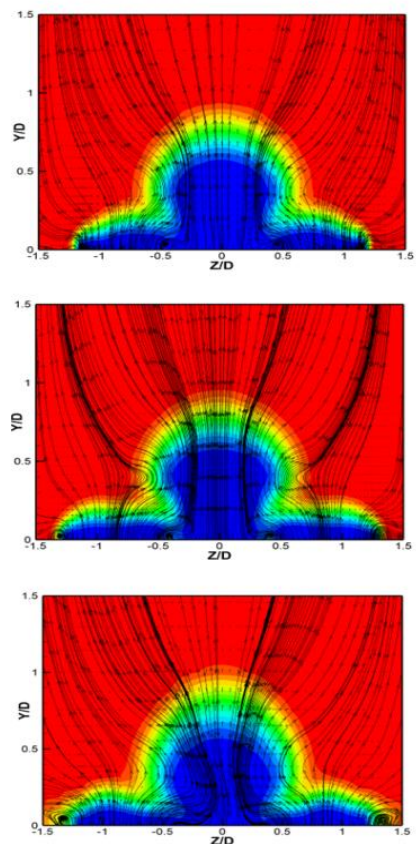

$\mathbf{X} / \mathbf{D}=\mathbf{1}$

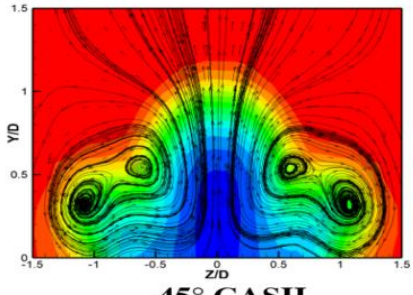

$45^{\circ}$ CASH

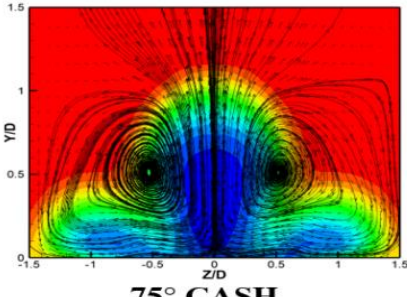

$75^{\circ} \mathrm{CASH}$

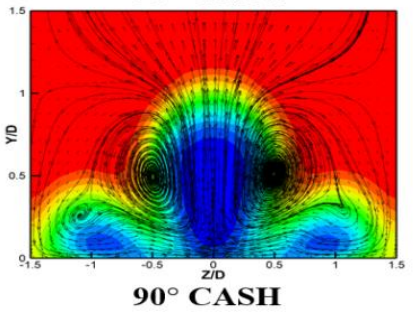

a)Midstream location

$\mathbf{X} / \mathbf{D}=\mathbf{1}$

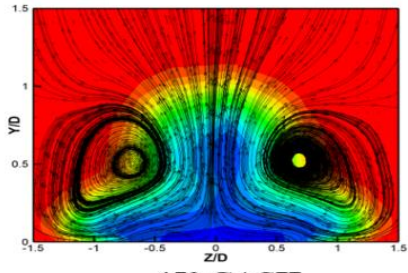

$45^{\circ}$ CASH

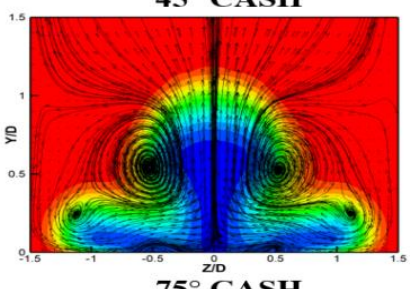

$75^{\circ}$ CASH

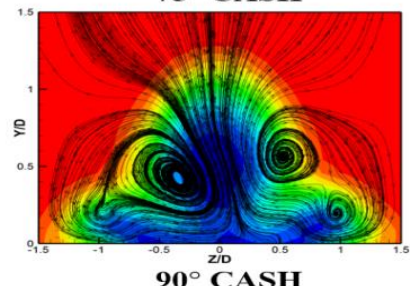

$\mathbf{X} / \mathbf{D}=\mathbf{5}$
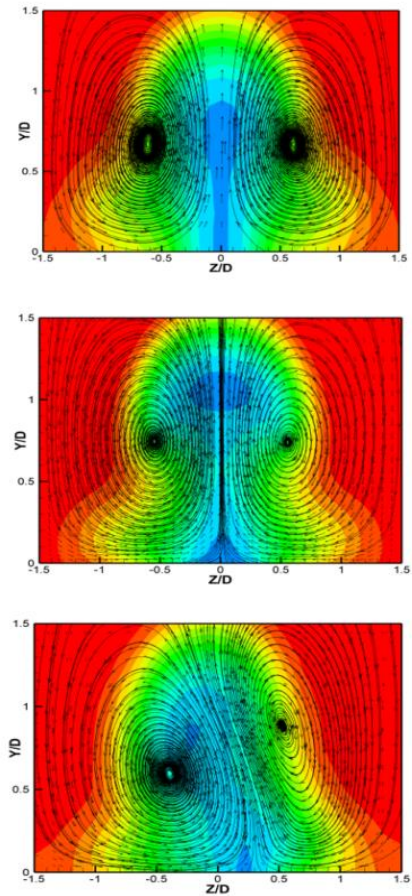

$\mathbf{X} / \mathbf{D}=\mathbf{5}$
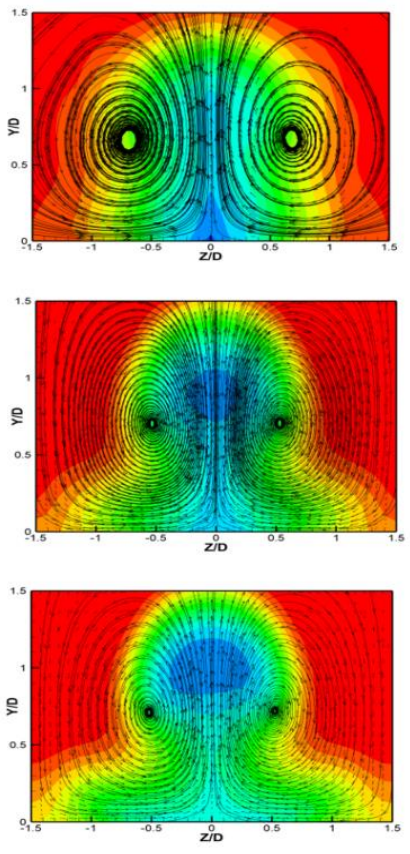

b) Downstream location

Temprature (K ) $150160170180190200210220230240250260270280290300 \longleftrightarrow$ \x Figure 7.13: The flow structure results of $\mathrm{CASH}$ at $\mathrm{X} / \mathrm{D}=\mathbf{0 , 1}$, and 5 a) midstream and b) downstream at $M=1.5$. 
ACRVP is noticeably stronger and works efficiently in controlling the CRVP. At $(\mathrm{X} / \mathrm{D}=1)$; however, the CRVP is developed and leads the flow while a bit small ACRVP exists, and there is somewhat of the asymmetry in the generated vortices pattern which drags the coolant flow to expand in the vertical direction. At $(X / D=5)$ further development of the ACRVP appears, and even though the coolant core is adhesion to the plate surface around the center, there is more mixing which caused the massive vertical expansion of the coolant flow. Overall, at X/D=0 and 1 , the lateral coolant coverage and distribution from the midstream location of the CASH is slightly better than that for the downstream location; however, at $\mathrm{X} / \mathrm{D}=5$, the best results are found through the downstream location of all CASH.

It is important to mention here that based on the advancement of todays manufacture technology and from the manufacturing point of view, compound angle cooling hole can be easily and economically manufactured through electrical discharge mechanism and/or laser drilling at any angle [28]. Also, cylindrical cooling holes are the most convenient and conventional shape of the cooling hole that being applied in real turbine blades and vanes for commercial and military aircraft's engines. Therefore, it can be said that the new hole configuration introduced herein in this thesis; compound angle sister holes are likely to be manufactured at low cost with less complexity and manufacturing time since all of the examined compound angle sister holes at all locations and regardless of the blowing ratios have shown notably high film cooling coverage and performance. Then, it can be employed to provide a more effective cooling protection for high-pressure turbine blades and vans of aero-engines and prevent the thermal failure precisely in the region that has high surface curvature.

\subsection{Conclusions}

In this chapter, the thermal performance of the compound angle sister holes at three different locations upstream, midstream, and downstream is evaluated at two blowing ratios $\mathrm{M}=0.5$ and $\mathrm{M}=1.5$.

In general, all of the examined locations for various CASH has shown a significant film cooling performance at low and high blowing ratios. However, at a low blowing ratio, $\mathrm{M}=0.5$, the optimum centerline effectiveness is accomplished by placing the compound angle sister hole downstream, while the best lateral film cooling effectiveness is achieved by (1) the midstream location of the CASH for the entire downstream area, and (2) by the downstream location of 
$\mathrm{CASH}$ for the hole vicinity region. At a high blowing ratio, $\mathrm{M}=1.5$, the downstream location offers the optimal centerline effectiveness up to $(\mathrm{X} / \mathrm{D} \approx 10)$, and the maximum lateral effectiveness is attained by the midstream location. Regardless of the blowing ratio and the sister hole location, compound angle sister holes provide a notable film cooling coverage in the holes adjacent region up to $(\mathrm{X} / \mathrm{D} \approx 10)$. However, at a high blowing ratio, the coolant coverage is deteriorated further downstream as a result of the intense mixing. The flow structure results of the compound angle sister holes present the generation of anti-counter rotating vortices pair (ACRVP) that plays a major role in controlling the flow field and avoiding the coolant lift-off phenomena at high blowing ratio. 


\section{CHAPTER EIGHT: CONCLUSIONS AND FUTURE WORK RECOMMENDATIONS}

This chapter presents the main findings and contributions obtained through comprehensive numerical investigations of film cooling performance of the micro holes and the novel compound angle sister holes. The parameters study includes the effect of such hole design and arrangements at different blowing ratios on centerline effectiveness, laterally averaged effectiveness, overall effectiveness, and flow structures. In addition, the chapter outlines the suggested recommendations for future work.

\subsection{Conclusions}

1. The film cooling performance assessment of the cylindrical micro hole with a $200 \mu \mathrm{m}$ diameter over a flat plate was done at low blowing ratios, $\mathrm{M}=0.25$ and $\mathrm{M}=0.5$, and high blowing ratios, $M=1.0$ and 1.5. The film cooling effectiveness results analysis; both centerline and laterally averaged effectiveness, demonstrated that the micro hole achieves optimum film cooling performance at low blowing ratios. In comparison to the cylindrical macro hole cooling performance, the micro hole offered a better lateral effectiveness and coolant spreading in the hole vicinity region up to $(X / D=5)$ whereby the highest averaged lateral effectiveness is attained with a $12 \%$ reduction in the coolant blowing ratio, which can be considered as saving in the coolant flow consumption. Moreover, the micro hole revealed higher overall averaged film, which is about $30 \%$ more than that of the macro hole. The analysis of flow field results of the velocity contours manifested that the coolant jet injected out of the micro hole has the tendency to stay attached to the plate surface at a low blowing ratio as well as to lift off and detached further downstream at high blowing ratios. The flow structure result analysis proved the presence of the counter-rotating vortices pair which produces a better lateral film cooling effectiveness and coolant distribution as a result of reduction of the CRVP strength in contrast to that of the macro hole. The effect of freestream turbulence intensity on the thermal performance of the round micro hole has also been evaluated whereby the numerical results indicated that increasing the freestream turbulence intensity to match the engine operating has insignificant effect at a low blowing ratio, while it intensifies the later film cooling performance at a high blowing ratio. 
2. CFD prediction ability of the three-dimensional complexed flow field of the sister holes film cooling has been determined at low and high blowing ratios in comparison with the experimental measurements of sister holes, using various RANS turbulence models: realizable k$\varepsilon$, standard k- $\varepsilon$, RNG k- $\varepsilon$, Reynolds stress model, and Spalart-Allmaras model, while all combined with the enhanced wall treatments. The laterally averaged film cooling effectiveness was used to judge the prediction capability of the turbulence models. At a low blowing ratio, the lateral effective results attained by realizable $\mathrm{k}-\varepsilon$ prediction are quantitatively in a good agreement to the experimental data. On the other hand, at a high blowing ratio, none of the examine turbulence models are able to capture the performance, and a consistent underprediction of the lateral performance is dominant from all turbulence models for the whole downstream region. In addition, the near-wall modeling effect is evaluated to determine the right approach that should be used to capture the lateral performance at $M=0.5$. The predicted results indicate that using the enhanced wall treatment is the suitable approach for a reasonable prediction of sister holes film cooling performance.

3. The film cooling performance is determined for a novel cooling hole scheme; compound angle upstream sister holes (CAUSH) injected at several compound angles at low and high blowing ratios in comparison to the sister holes that are only injected to the streamwise whereas $\beta=0^{\circ}$ as well as to a discrete cylindrical hole. The results of the centerline and lateral film cooling performance revealed a considerable increase in the performance, specifically, at high blowing ratios for the whole downstream region than that obtained from streamwise sister holes. The compound angle upstream sister holes technique is found to offer viable improvements over standard single hole whereas the overall cooling performance is increased by a factor of 2.0, 1.97, 1.91, and 2.26 for the $0^{\circ}, 45^{\circ}, 75^{\circ}$, and $90^{\circ} \mathrm{CAUSH}$, respectively. The detailed evaluation of the flow field results of CAUSH confirmed the presence of the anti-contour rotating vortices pair (ACRVP) that contributed heavily to the attained high cooling performance whereby the ACRVP is significantly maintained the coolant jet adhesion to the plate surface even at high blowing ratios and suppress the jet lift-off. Also, the ACRVP are located apart from each other close to the computational domain boundary; as a result, a better and uniform lateral coolant spreading is obtained for all examined blowing ratios. It is found that the optimum centerline and lateral performance is accomplished by $90^{\circ} \mathrm{CAUSH}$ at a blowing ratio of $\mathrm{M}=1.0$, while overall, the 
proposed compound angle upstream sister holes offer significant thermal protection for all examined blowing ratios.

4. A further investigation is performed for the compound angle sister holes to determine the effect of the streamwise locations of the compound angle sister holes on film cooling performance and flow field whereby three locations: upstream, midstream, and downstream, are numerically examined for several compound angles at $\beta=45^{\circ}, 75^{\circ}$, and $90^{\circ}$ at only two blowing ratios $M=0.5$ and 1.5. The analysis of the centerline film cooling effectiveness demonstrated that the upstream location was not the optimal place to achieve maximum performance. Moreover, regardless of the blowing ratio and the compound angle, placing the sister holes in the downstream location provided the highest centerline performance compared to the other locations. On the other hand, placing the sister holes in the midstream location, whereas the center of the compound sister holes was aligned with the center of the main hole, allowed to obtain better lateral effectiveness. Furthermore, it was found that by comparing the cooling performance of the all examined compound angle sister holes locations at low blowing ratio, the optimum centerline and lateral effectiveness are accomplished by $45^{\circ} \mathrm{CASH}$ downstream and $75^{\circ} \& 90^{\circ} \mathrm{CASH}$ midstream, respectively. While at high blowing ratio, the optimum centerline and lateral effectiveness are obtained by $45^{\circ} \mathrm{CASH}$ downstream and $90^{\circ} \mathrm{CASH}$ midstream, respectively. The investigation of the flow structure results demonstrated that the midstream location had shown the greatest lateral performance because of the strong ACRVP vortices that sustain a considerable amount of coolant core adhesion to the plate surface with less penetration into the freestream. The notable film cooling performance has been accomplished by CASH over a wide range of blowing ratios promoting it to be effectively applied and implemented by engine manufacturers to prevent the thermal failure of high-pressure turbine blades and vans; precisely in the region that has a high surface curvature, while taking the advantage of being easily manufactured since it consists only of a traditional cylindrical holes.

\subsection{Contributions of the Present Research}

The contributions of the present research effort to the existing state of the art literature on film cooling might be summarized as follows: 
1. Micro holes film cooling detailed numerical assessment is the first in the literature to show the great benefits that can be obtained from using such a technique that appeared on the increase in film cooling effectiveness and a reduction on the coolant flow usage. The numerical results were validated and compared to a discrete macro cylindrical cooling hole at the same operating conditions.

2. The numerical analysis of micro holes film cooling has presented that micro holes lateral averaged film cooling effectiveness can be increased further by using high freestream turbulence intensity at a high blowing ratio.

3. The numerical evaluation of the RANS turbulence models prediction of the upstream sister holes film cooling effectiveness and local film cooling distribution in comparison to the only existing experimental data is documented for the first time in the current work.

4. The numerical results show a significant potential of applying relizable k-epsilon turbulence model along with the enhanced wall treatment to well predict the lateral film cooling effectiveness and local film cooling distribution at low blowing ratios. On the other hand, none of the examined turbulence models has shown its ability to predict the laterally averaged effectiveness at high blowing ratios and presented a significant underprediction.

5. The novel cooling hole scheme proposed herein " compound angle sister upstream sister holes" has shown through extensive numerical analysis a considerable improvement in the film cooling effectiveness at low and high blowing ratios in contrast to the single round hole. In addition, compound angle upstream sister holes have effectively controlled the flow field and suppressed the jet lift off at high blowing ratios.

6. The modification of the compound angle sister holes streamwise location has caused a notable variation in the film cooling effectiveness performance whereas it was found that, regardless of the blowing ratio and the compound angle, the best centerline and lateral effectiveness are offered by the downstream location and the midstream location, respectively.

\subsection{Future Work Recommendations}

The present research may be expanded based on the following future work recommendations:

1. The numerical results of the micro hole have shown an improvement in the overall film cooling performance. This improvement should pave the ground and encourage for more parametric 
experiments. It is to note that dealing with the micro size of the cooling increases the risk of blocking the cooling holes by the sand or volcanic ash which will adversely affect the cooling performance; therefore, the analysis of the micro cooling deposition is recommended both numerically and experimentally.

2. The sister holes cooling performance is computationally predicted through the RANS turbulence models, for more accurate and detailed flow field numerical results; advanced turbulence models are recommended such large eddy simulation or direct numerical simulation.

3. Perform an extensive parametric experimental study on the proposed compound angle sister holes to study the effect of cooling hole length to diameter ratio, pitch to diameter ratio, the distance between the holes both spanwise and streamwise, and the injection angle $(\alpha)$ on the cooling performance and flow field.

4. The compound angle sister holes configuration reveals a significant enhancement on the cooling performance, whereas the sister holes are systemically oriented to the freestream direction while the main hole is injected to the streamwise. It would be interesting to evaluate the cooling performance if both the main hole and the sister holes are injected at the same compound angle.

5. Investigate the cooling performance of the micro hole and compound angle sister holes on a turbine blade leading edge since the current assessment is limited to the analysis over a flat plate.

6. Combine the compound angle sister holes, and the micro hole in one hybrid cooling hole scheme would also be interesting, whereas the sister hols will be replaced by micro size cooling holes and this idea will reduce the coolant flow usage. 


\section{REFERENCES}

[1] J.-C. Han, "Fundamental Gas Turbine Heat Transfer," J. Therm. Sci. Eng. Appl., vol. 5, no. 2, pp. 021007-021007-15, 2013.

[2] J.-C. Han, S. Dutta, and S. Ekkad, Gas Turbine Engine heat transfer and cooling Technology, 2nd ed. London NewYork: CRC Press, 2012.

[3] J. P. Downs and K. K. Landis, "Turbine Cooling Systems Design: Past, Present and Future," in Proceedings of ASME Turbo Expo 2009, No. GT2009-59991, 2009, pp. 819828.

[4] J.-C. Han, "Advanced Cooling in Gas Turbines 2016 Max Jakob Memorial Award Paper," J. Heat Transfer, vol. 140, no. 11, pp. 113001-20, 2018.

[5] S. Naik, "Basic Aspects of Gas Turbine Heat Transfer," in Heat Exchangers- Design, Experiment and Simulation, S. M. S. Murshed and M. M. Lopes, Eds. IntechOpen, Rijeka, 2017, pp. 111-142.

[6] B. Lakshminarayana, Fluid Dynamics and Heat Transfer of Turbomachinery. John Wiley \& Sons, New York, USA. Dixon, 1996.

[7] R. S. Bunker, "Evolution of turbine cooling," in Proceedings of ASME Turbo Expo 2017, No. GT2017-63205, 2017, p. V001T51A001-26.

[8] R. S. Bunker, "Turbine cooling design analysis," in Gas Turbine Handbook - NETL, 2006, pp. 295-309.

[9] T. I.-P. Shih and V. Yang, Turbine aerodynamics, heat transfer, materials, and mechanics. American Institute of Aeronautics and Astronautics, Inc., 2014.

[10] H. Wu, H. Cheng, Y. Li, C. Rong, and S. Ding, "Effects of side hole position and blowing ratio on sister hole film cooling performance in a flat plate," Appl. Therm. Eng., vol. 93, pp. 718-730, 2016.

[11] G. Cerri, A. Giovannelli, L. Battisti, and R. Fedrizzi, "Advances in effusive cooling techniques of gas turbines," Appl. Therm. Eng., vol. 27, no. 4, pp. 692-698, 2007.

[12] R. S. Bunker, "Turbine Cooling: Moving From Macro To Micro Cooling," in Proceedings of ASME Turbo Expo 2013: Turbine Technical Conference and Exposition GT2013 GAS, 
2013, pp. 1-17.

[13] D. S. DUVALL, M. P. Development, U. T. Corporation, and E. Hartford, "The Use of Lasers in Gas Turbine Manufacturing," in The American Society of Mechanical Engineers, 1985, pp. 1-7.

[14] C. A. McNally, J. Folkes, and I. R. Pashby, "Laser drilling of cooling holes in aero-engines: state of the art and future challenges," Mater. Sci. Technol., vol. 20, no. 7, pp. 805-813, 2004.

[15] “aerospace_manufacturing_magazine_july_2008.pdf," It all adds up for engines, 2017. .

[16] J. Green, "Micro-Scale Film Cooling for Gas Turbine Applications," MSc Thesis, Aerospace engineering Department, Cranfield University, UK, 2007.

[17] R. Sriram and G. Jagadeesh, "Film cooling at hypersonic Mach numbers using forward facing array of micro-jets," Int. J. Heat Mass Transf., vol. 52, no. 15-16, pp. 3654-3664, 2009.

[18] M. Barzegar Gerdroodbary, M. Imani, and D. D. Ganji, "Investigation of film cooling on nose cone by a forward facing array of micro-jets in Hypersonic flow," Int. Commun. Heat Mass Transf., vol. 64, pp. 42-49, 2015.

[19] P. L. Li, H. S. Ko, D. Z. Jeng, C. W. Liu, and C. Gau, "Micro film cooling performance," Int. J. Heat Mass Transf., vol. 52, no. 25-26, pp. 5889-5894, 2009.

[20] O. Hassan and I. Hassan, "Experimental investigations of the film cooling effectiveness of a micro-tangential-jet scheme on a gas turbine vane," Int. J. Heat Mass Transf., vol. 61, no. 1, pp. 158-171, 2013.

[21] O. Hassan, "Thermal and Flow Field Investigations of a Micro- Tangential-Jet Film Cooling Scheme on Gas Turbine Components," Ph.D.Thesis, Department of Mechanical and Industrial Engineering, Concordia University Montreal, Quebec, Canada, 2013.

[22] O. Hassan and I. Hassan, "Experimental Flow-Field Investigations Downstream a ScaledUp Micro-Tangential-Jet Scheme Using the Particle Image Velocimetry Technique," J. Fluids Eng., vol. 136, no. 7, p. 071204, 2014.

[23] S. Balasubramaniyan and B. A. Jubran, "Numerical analysis of film cooling from micro- 
hole,” Springer Berlin Heidelberg, 2013.

[24] S. Balasubrammaniyan, "Numerical Analysis of Film Cooling from Micro- Hole.," Master of Engineering Project, Department of Aerospace Engineering, Ryerson University, Toronto, Canada, 2013.

[25] T. Ochrymiuk, "Numerical analysis of microholes film/effusion cooling effectiveness," $J$. Therm. Sci., vol. 26, no. 5, pp. 459-464, 2017.

[26] R. J. Goldstein, "Film Cooling,” Adv. Heat Transf., vol. 7, pp. 321-379, 1971.

[27] D. G. Bogard and K. A. Thole, "Gas Turbine Film Cooling," J. Propuls. Power, vol. 22, no. 2, pp. 249-270, 2006.

[28] R. S. Bunker, "A Review of Shaped Hole Turbine Film-Cooling Technology," J. Heat Transfer, vol. 127, no. 4, pp. 441-453, 2005.

[29] R. J. Goldstein, E. R. G. Eckert, and J. W. Ramsey, "Film Cooling With Injection Through Holes: Adiabatic Wall Temperatures Downstream of a Circular Hole," J. Eng. Gas Turbines Power, vol. 90, no. 4, pp. 384-393, 1968.

[30] R. J. Goldstein, E. R. G. Eckert, and F. Burggraf, "Effects of Hole Geometry and Density on Three-Dimensional Film Cooling," Int. J. Heat Mass Transf., vol. 17, pp. 595-607, 1974.

[31] K. Singh, B. Premachandran, and M. R. Ravi, "Experimental assessment of film cooling performance of short cylindrical holes on a flat surface," Heat Mass Transf. Waerme- und Stoffuebertragung, vol. 52, no. 12, pp. 2849-2862, 2016.

[32] J.H. Leylek and R.D. Zerkle, "Discrete-Jet Film Cooling: A Comparison of Computational Results with Experiments,93-GT-207," in The American Society of Mechanical Engineers, 1993, pp. 1-14.

[33] E. Lutum and B. V. Johnson, "Influence of the Hole Length-to-Diameter Ratio on Film Cooling With Cylindrical Holes," J. Turbomach., vol. 121, no. 2, p. 209, 1999.

[34] A. Azzi and B. A. Jubran, "Numerical modeling of film cooling from short length streamwise injection holes," Heat Mass Transf., vol. 39, no. 4, pp. 345-353, 2003.

[35] N. W. Foster and D. Lampard, "The Flow and Film Cooling Effectiveness Following 
Injection through a Row of Holes," J. Eng. Power, vol. 102, no. 3, pp. 584-588, Jul. 1980.

[36] A. Kohli and D. G. Bogard, "Adiabatic effectiveness, thermal fields, and velocity fields for film cooling with large angle injection," J. Turbomach., vol. 119, no. 2, pp. 352-358, 1997.

[37] S. Baldauf, A. Schulz, and S. Wittig, "High-Resolution Measurements of Local Heat Transfer Coefficients From Discrete Hole Film Cooling," J. Turbomach., vol. 123, no. 4, p. 749, 2001.

[38] S. M. Abd Alsalam and B. A. Jubran, "A Parametric CFD Study of Film Cooling Effectiveness for a Discrete Short Cooling Hole on a Flat Plate.," in Proceedings of the 63th Canadian Aeronautics and Space Institute (CASI AERO 2017) Conference, Toronto, Ontario, Canada., 2017, no. 1, pp. 1-16.

[39] A. Roshko, T. F. Fric, and A. Roshko, "Vortical structure in the wake of a transverse jet," J. Fluid Mech., vol. 279, pp. 1-47, 1994.

[40] S. H. Smith and M. G. Mungal, "Mixing, structure and scaling of the jet in crossflow," $J$. Fluid Mech., vol. 357, pp. 83-122, 1998.

[41] D. K. Walters and J. H. Leylek, "A detailed analysis of film cooling physics part I: streamwise injection with cylindrical holes," J. Turbomach., vol. 122, no. 1, pp. 102-112, 1997.

[42] K. T. McGovern and J. H. Leylek, “A Detailed Analysis of Film Cooling Physics: Part IICompound-Angle Injection With Cylindrical Holes," J. Turbomach., vol. 122, no. 1, pp. $113-121,2000$.

[43] S. Bernsdorf, M. G. Rose, and R. S. Abhari, "Modeling of Film Cooling-Part I: Experimental Study of Flow Structure," J. Turbomach., vol. 128, no. 1, pp. 141-149, 2005.

[44] H. M. Li and I. Hassan, "The Effects of Counterrotating Vortex Pair Intensity on FilmCooling Effectiveness," Heat Transf. Eng., vol. 36, no. 16, pp. 1360-1370, 2015.

[45] H. Li, W. Ghaly, and I. Hassan, "The Formation of Counter-Rotating Vortex Pair and the Nature of Liftoff-Reattachment in Film-Cooling Flow," Fluids, vol. 1, no. 4, p. 39, 2016.

[46] S. Acharya and Y. Kanani, "Advances in Film Cooling Heat Transfer," in Advances in Heat Transfer, 1st ed., vol. 49, Elsevier Inc., 2017, pp. 91-156. 
[47] D. L. Schmidt, B. Sen, and D. G. Bogard, "Film cooling with compound angle holes: adiabatic effectiveness," J. Turbomach., vol. 118, no. 4, pp. 807-813, 1996.

[48] P. M. Ligrani and J. S. Lee, "Film Cooling from a Single Row of Compound Angle Holes at High Blowing Ratios," Int. J. Rotating Mach., vol. 2, no. 3, pp. 201-208, 1996.

[49] S. Ekkad, D. Zapata, and J. Han, "Film effectiveness over a flat surface with air and C02 injection through compound angle holes using a transient liquid crystal image method," $J$. Turbomach., vol. 119, no. 3, pp. 587-593, 1997.

[50] A. Azzi, M. Abidat, B. A. Jubran, and G. S. Theodoridis, "Film cooling predictions of simple and compound angle injection from one and two staggered rows," Numer. Heat Transf. Part A Appl., vol. 40, no. 3, pp. 273-294, 2001.

[51] V. Sharma and A. Garg, "Numerical Investigation of Effects of Compound Angle and Length to Diameter Ratio on Adiabatic Film Cooling Effectiveness,” 2014.

[52] G. Natsui et al., "Experimental Evaluation of Large Spacing Compound Angle FullCoverage Film Cooling Arrays: Adiabatic Film Cooling Effectiveness," J. Turbomach., vol. 138, no. 7, p. 071001, 2016.

[53] M. H. Bashir, C. Shiau, and J. Han, "Film cooling effectiveness for three-row compound angle hole design on flat plate using PSP technique," Int. J. Heat Mass Transf., vol. 115, pp. 918-929, 2017.

[54] W. Li, X. Lu, X. Li, J. Ren, and H. Jiang, "High resolution measurements of film cooling performance of simple and compound angle cylindrical holes with varying hole length-todiameter ratio - Part I : Adiabatic film effectiveness," Int. J. Therm. Sci., vol. 124, pp. 146-161, 2018.

[55] R. B. A., J. H. Leylek, D. G. Hyams, and J. H. Leylek, "A Detailed Analysis of Film Cooling Physics: Part III—Streamwise Injection With Shaped Holes," J. Turbomach., vol. 122, no. 1, p. 122, 2002.

[56] S. Baheri, S. P. A. Tabrizi, and B. A. Jubran, "Film cooling effectiveness from trenched shaped and compound holes," Heat Mass Transf., vol. 44, no. 8, pp. 989-998, 2008.

[57] S. Haydt and S. Lynch, "Cooling Effectiveness for a Shaped Film Cooling Hole at a Range 
of Compound Angles,” J. Turbomach., vol. 141, no. 4, pp. 041005-14, 2019.

[58] R. S. Bunker, "Film Cooling: Breaking the Limits of Diffusion Shaped Holes," Heat Transf. Res., vol. 41, no. 6, pp. 627-650, 2010.

[59] A. Javadi, K. Javadi, M. Darbandi, M. Taeibi-Rahni, and M. Darbandi, “A New Approach to Improve Film Cooling Effectiveness Using Combined Jets,” Momentum, vol. 2, p. 2, 2003.

[60] K. Javadi, M. Taeibi-Rahni, and M. Darbandi, "Jet-into-crossflow boundary-layer control: Innovation in gas turbine blade cooling," Aiaa J., vol. 45, no. 12, pp. 2910-2925, 2007.

[61] M. J. Ely and B. A. Jubran, “A Numerical Study on Increasing Film Cooling Effectiveness Through the Use of Sister Holes," in Proceedings of ASME Turbo Expo 2008: Power for Land, Sea and Air, No, GT2008-50366, 2008, pp. 341-350.

[62] M. J. Ely and B. A. Jubran, "A numerical study on improving large angle film cooling performance through the use of sister holes," Numer. Heat Transf., vol. 55, no. 7, pp. 634653, 2009.

[63] M. J. Ely and B. A. Jubran, "A numerical evaluation on the effect of sister holes on film cooling effectiveness and the surrounding flow field," Heat Mass Transf., vol. 45, no. 11, pp. 1435-1446, 2009.

[64] M. J. Ely and B. A. Jubran, "A parametric study on the effect of sister hole location on active film cooling flow control," in Proceedings of ASME Turbo Expo 2010: Power for Land, Sea, and Air, No. GT2010-22060, 2010, pp. 1301-1311.

[65] M. J. Ely and B. A. Jubran, "Film Cooling From Short Holes with Sister Hole Influence," in Proceedings of ASME Turbo Expo 2012, No. GT2012-68081, 2012, pp. 1-12.

[66] S. Khajehhasani and B. Jubran, "A CFD-Based Parametric Study on Modification of Discrete Sister Holes Location for the Film Cooling Flow," in Proceedings of ASME Turbo Expo 2014, No. GT2014-25970, 2014, p. V05BT13A033-1-11.

[67] S. Khajehhasani and B. A. Jubran, "A Numerical Evaluation of the Performance of Film Cooling from a Circular Exit Shaped Hole with Sister Holes Influence," Heat Transf. Eng., vol. 37, no. 2, pp. 183-197, 2016. 
[68] S. Khajehhasani and B. A. Jubran, "Numerical assessment of the film cooling through novel sister-shaped single-hole schemes," Numer. Heat Transf., vol. 67, no. 4, pp. 414-435, 2015.

[69] S. J. Dai, Y. Xiao, L. M. He, T. Jin, and Z. C. Zhao, "Film cooling from a cylindrical hole with parallel auxiliary holes influences," Numer. Heat Transf. Part A Appl., vol. 69, no. 5, pp. 497-511, 2016.

[70] J.H. Leylek et al., "Discrete-Jet Film Cooling: A Comparison of Computational Results With Experiments," J. Turbomach., vol. 116, no. 3, pp. 358-368, 1993.

[71] Jubran B., A. A. Amer, B. A. Jubran, and M. A. Hamdan, "Comparison of Different TwoEquations Turbulence models for Prediction of Film Cooling from Two Rows of Holes," Numer. HEAT Transf. PART A, vol. 21, no. 2, pp. 143-162, 1992.

[72] D. K. Walters and J. H. Leylek, "A Systematic Computational Methodology Applied to a Three-Dimensional Film-Cooling Flowfield," J. Turbomach., vol. 119, no. 4, pp. 777$785,1997$.

[73] A. Azzi and D. Lakehal, "Perspectives in Modeling Film Cooling of Turbine Blades by Transcending Conventional Two-Equation Turbulence Models," J. Turbomach., vol. 124, no. 3, pp. 472-484, 2002.

[74] S. Na, B. Zhu, M. Bryden, and T. I.-P. Shih, "CFD Analysis of Film Cooling," in 44th AIAA Aerospace Sciences Meeting and Exhibit, 2006, pp. 1-10.

[75] J. S. Hassan and S. Yavuzkurt, "Comparison of Four Different Two-Equation Models of Turbulence in Predicting Film Cooling Performance," in Proceedings of ASME Turbo Expo 2006: Power for Land, Sea, and Air, No. GT2006-90860, 2006, pp. 1-10.

[76] K. L. Harrison and D. G. Bogard, "Comparison of RANS Turbulence Models for Prediction of Film Cooling Performance," in AProceedings of ASME Turbo Expo 2008, NO. GT200851423, 2008, vol. 4, no. 43147, pp. 1-10.

[77] X. Li, J. Ren, and H. Jiang, “Application of algebraic anisotropic turbulence models to film cooling flows," Int. J. Heat Mass Transf., vol. 91, pp. 7-17, 2015.

[78] S. Khajehhasani and B. A. Jubran, "A numerical investigation of film cooling performance through variations in the location of discrete sister holes," Appl. Therm. Eng., vol. 107, pp. 
345-364, 2016.

[79] C. El Ayoubi, "Three-Dimensional Aero-Thermal Optimization of Film Cooling in a High Pressure Turbine," $\mathrm{PhD}$ thesis, Department of Mechanical and Industrial Engineering, Concordia University, Montreal, Quebec, Canada., 2014.

[80] D. C. Wilcox, Turbulence Modeling for CFD, 3rd ed. DCW Industries Inc., 2006.

[81] ANSYS Inc., ANSYS FLUENT Theory Guide, Release 15. Canonsburg, PA, 275 Technology Drive Canonsburg, PA 15317, 2013.

[82] ANSYS Inc., "Turbulence Modeling Introduction: Introduction to ANSYS Fluent," ANSYS Customer Training Material, no. December. pp. 1-59, 2010.

[83] B. E. Launder and D. B. Spalding, "Lectures in mathematical models of turbulence ." Academic Press , 1972.

[84] V. Yakhot and S. A. Orszag, "Renormalization group analysis of turbulence. I. Basic theory," J. Sci. Comput., vol. 1, no. 1, pp. 3-51, 1986.

[85] ANSYS Inc., ANSYS FLUENT User's Guide, Release 13. Canonsburg, PA, 275 Technology Drive Canonsburg,PA 15317, 2010.

[86] T. H. Shih, W. W. Liou, A. Shabbir, Z. Yang, and J. Zhu, "A new k- $\varepsilon$ eddy viscosity model for high reynolds number turbulent flows," Comput. Fluids, vol. 24, no. 3, pp. 227-238, 1995.

[87] G. Kalitzin, G. Medic, G. Iaccarino, and P. Durbin, "Near-wall behavior of RANS turbulence models and implications for wall functions," J. Comput. Phys., vol. 204, no. 1, pp. 265-291, 2005.

[88] B. E. Launder and D. B. Spalding, "The Numerical Computation of Turbulent Flows," Comput. Methods Appl. Mech. Eng., vol. 3, pp. 269-289, 1974.

[89] A. K. Sinha, D. G. G. Bogard, and M. E. E. Crawford, "Film-cooling effectiveness downstream of a single row of holes with variable density ratio," J. Turbomach., vol. 113, no. 3, pp. 442-449, 1991.

[90] N. G. Hadjiconstantinou, "The limits of Navier-Stokes theory and kinetic extensions for describing small-scale gaseous hydrodynamics," Phys. Fluids, vol. 18, no. 11, pp. 1-19, 
2006.

[91] S. Kakaç, Y. Yaman, and A. Pramuanjaroenkij, Convective heat transfer, Third edit. Boca Raton, [Florida] CRC Press, Taylor \& Francis Group, 2014.

[92] R. W. Barber and D. Emerson, "The influence of Knudsen number on the hydrodynamic development length within parallel plate micro-channels," Adv. fluid Mech., vol. 32, pp. 207-216, 2002.

[93] P. Bradshaw and G. P. Huang, "The law of the wall in turbulent flow," Proc. Math. Phys. Sci., vol. 451, no. 1941, pp. 165-188, 1995.

[94] X. Z. Zhang and I. Hassan, "Film Cooling Effectiveness for an Advanced-Louver Cooling Scheme for Gas Turbines," J. Thermophys. Heat Transf., vol. 20, no. 4, pp. 754-763, 2006.

[95] S. Abd Alsalam and B. Jubran, "Numerical Evaluation of Film Cooling Performance of a Cylindrical Micro Hole with the Influence of the Blowing Ratio," in Proceedings of the 26st Annual Conference of CFD Society of Canada, Winnipeg, Manitoba, Canada., 2018, pp. $1-11$.

[96] P. L. Johnson, V. Shyam, and C. Hah, "Reynolds-Averaged Navier-Stokes Solutions to Flat Plate Film Cooling Scenarios," Natl. Aeronaut. Sp. Adm. Glenn Res. Cent., no. No.NasaTm-217025, pp. 1-12, 2011.

[97] S. Khajehhasani, "Numerical Modeling of Innovative Film Cooling Hole Schemes," PhD thesis, Aerospace engineering, Ryerson University, 2014.

[98] D.R.Pedersen et al., "Film Cooling With Large Density Differences Between the Mainstream and the Secondary Fluid Measured by the Heat-Mass Transfer Analogy," ASME J. Heat Transf., vol. 99, no. November, pp. 620-627, 1977.

[99] B. Johnson, W. Tian, K. Zhang, and H. Hu, “An experimental study of density ratio effects on the film cooling injection from discrete holes by using PIV and PSP techniques," Int. J. Heat Mass Transf., vol. 76, pp. 337-349, 2014.

[100] A. Kohli and D. G. Bogard, "Effects of very high free-stream turbulence on the jetmainstream interaction in a film cooling flow," J. Turbomach., vol. 120, no. October 1998, pp. 785-790, 1998. 
[101] S. Abd Alsalam and B. Jubran, "Film Cooling of Compound Angle Upstream Sister Holes," in Proceedings of ASME Turbo Expo 2019, No. GT2019-90518, 2019, pp. 1-13.

[102] K. L. Harrison and D. G. Bogard, "CFD Predictions of Film Cooling Adiabatic Effectiveness for Cylindrical Holes Embedded in Narrow and Wide Transverse Trenches," in Proceedings of ASME Turbo Expo 2007, No. GT2007-28005, 2007, pp. 1-10.

[103] Z. T. Stratton and T. I.-P. Shih, "Identifying Weaknesses in Eddy-Viscosity Models for Predicting Film Cooling via Large-Eddy Simulations,” J. Propuls. Power, vol. 35, no. 3, pp. 583-594, 2019.

[104] I. S. Jung and J. S. Lee, "Effects of Orientation Angles on Film Cooling Over a Flat Plate: Boundary Layer Temperature Distributions and Adiabatic Film Cooling Effectiveness," J. Turbomach., vol. 122, no. 1, pp. 153-160, 2000. 